INEL-96/0249

Rev. 1

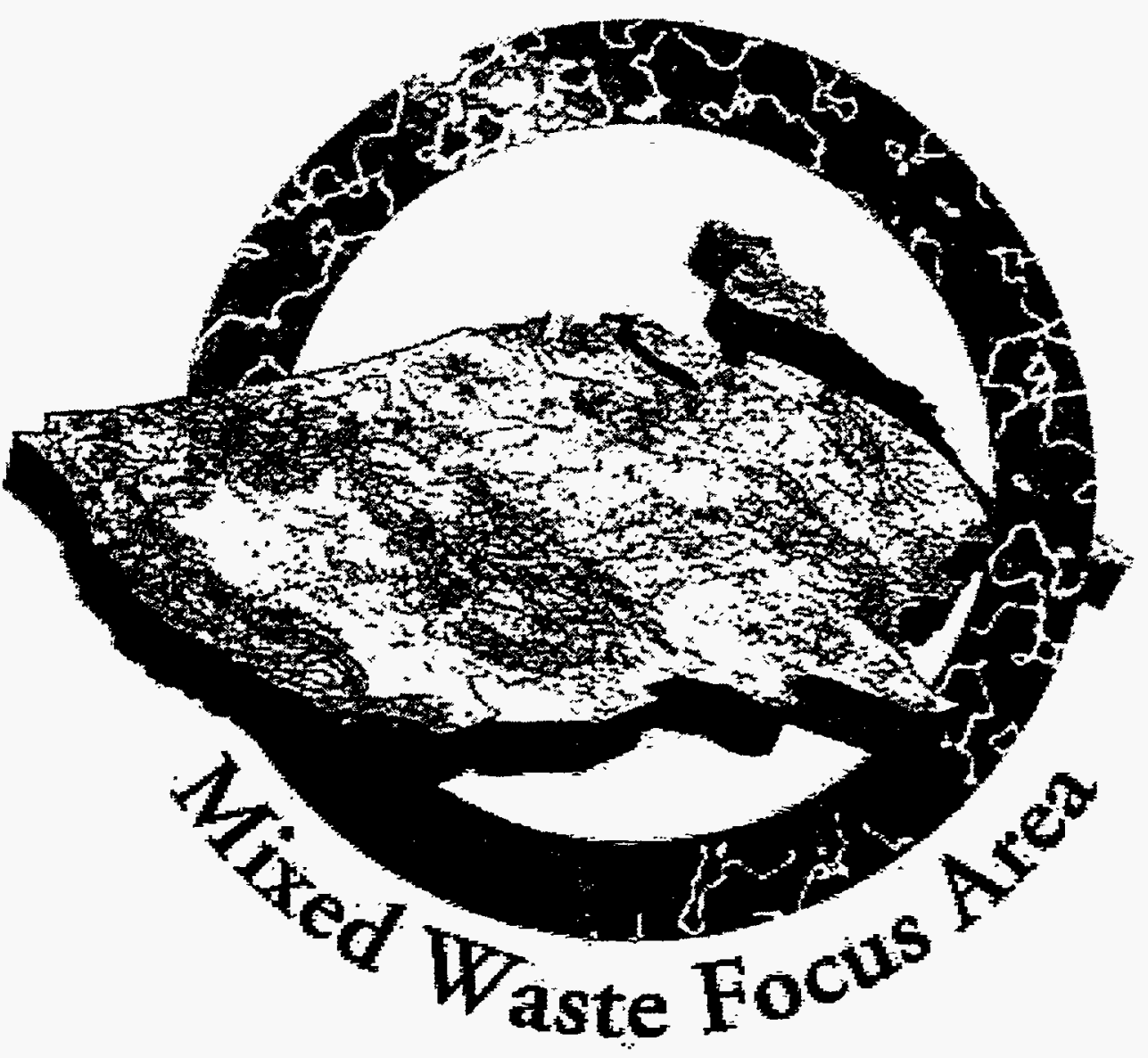

\title{
Commercial Treatability Study \\ Capabilities for Application to the U.S. Department of Energy's Anticipated Mixed Waste Streams
}

DISTRIEUTION OF THIS DOCUMENT IS URLIFATED 


\title{
Commercial Treatability Study Capabilities for Application to the U.S. Department of Energy's Anticipated Mixed Waste Streams
}

Published September 1996

Revision 1

\author{
IT Corporation \\ 5301 Central Ave. NW \\ Albuquerque, NM 87108
}
Prepared for Lockheed Idaho Technologies Company Mixed Waste Focus Area and for the U.S. Department of Energy Assistant Secretary for Environmental Management Under DOE Idaho Operations Office Contract DE-AC07-94ID13223




\section{DISCLAIMER}

Portions of this document may be illegible in electronic image products. Images are produced from the best available original document. 


\section{DISCLAIMER}

This report was prepared as an account of work sponsored by an agency of the United States Government. Neither the United States Government nor any agency thereof, nor any of their employees, makes any warranty, express or implied, or assumes any legal liability or responsibility for the accuracy, completeness, or usefulness of any information, apparatus, product, or process disclosed, or represents that its use would not infringe privately owned rights. Reference herein to any specific commercial product, process, or service by trade name, trademark, manufacturer, or otherwise does not necessarily constitute or imply its endorsement, recommendation, or favoring by the United States Government or any agency thereof. The views and opinions of authors expressed herein do not necessarily state or reflect those of the United States Government or any agency thereof. 


\section{TABLE OF CONTENTS}

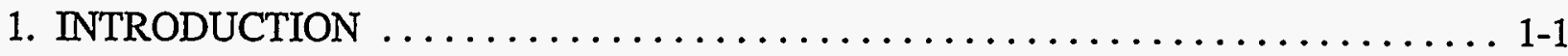

2. TREATABILITY STUDY SURVEY RESULTS $\ldots \ldots \ldots \ldots \ldots \ldots \ldots \ldots \ldots \ldots \ldots$

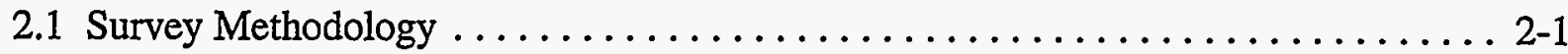

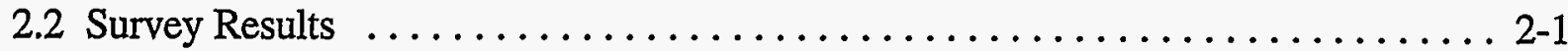

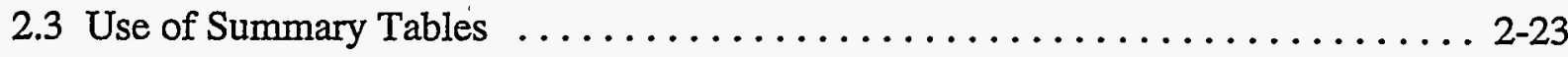

3. REGULATORY PROVISIONS ......................... 3-1

3.1 Resource Conservation and Recovery Act . . . . . . . . . . . . . . 3-1

3.1.1 The Treatability Study Exemption $\ldots \ldots \ldots \ldots \ldots \ldots \ldots \ldots \ldots \ldots . \ldots \ldots \ldots$

3.1.2 Implications of Hazardous Waste Determination $\ldots \ldots \ldots \ldots \ldots \ldots \ldots$. . . . .

3.1.3 Generator Requirements . ....................... 3-4

3.2 Toxic Substances Control Act . . . . . . . . . . . . . . . . . . . 3-5

3.2.1 Applicability to Research and Development Facilities . . . . . . . . . 3-5

3.2.2 Exemptions for Research and Development Facilities . . . . . . . . . 3-6

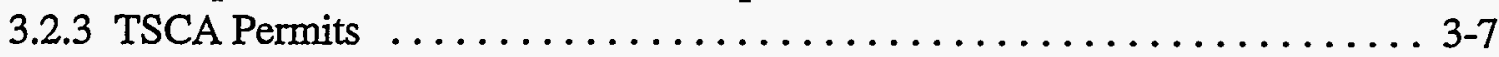

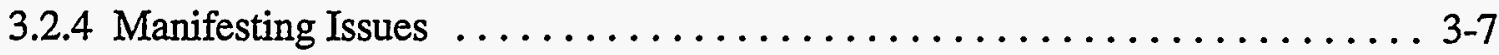

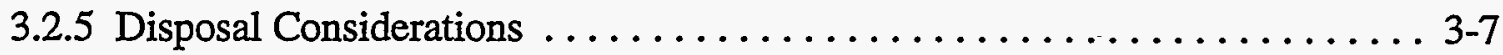

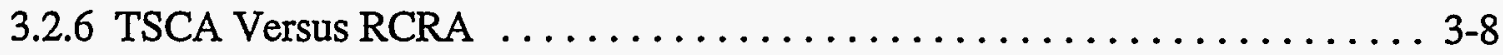

3.3 CERCLA Off-Site Rule . . . . . . . . . . . . . . . . . . . . 3-9

3.4 Nuclear Regulatory Commission Summary . . . . . . . . . . . . . . . . 3-10

3.4.1 Commercial Treatability Study Facility License Requirements . . . . . . . . 3-10

3.4.2 Packaging and Transportation of Radioactive Material . . . . . . . . . 3-11

3.5 U.S. Department of Transportation Summary and Guidance . . . . . . . . . . . 3-12

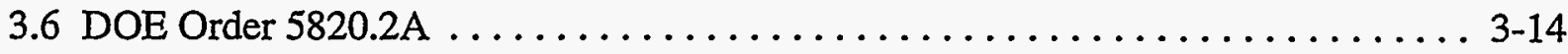

3.7 National Environmental Policy Act . . . . . . . . . . . . . . . 3-14

4. TREATABILITY STUDY MANAGEMENT ISSUES $\ldots \ldots \ldots \ldots \ldots \ldots \ldots \ldots \ldots$

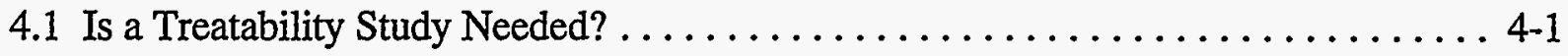

4.2 Develop Data Quality Objectives $\ldots \ldots \ldots \ldots \ldots \ldots \ldots \ldots \ldots \ldots \ldots \ldots \ldots \ldots$

4.3 Are Waste Characterization Data Adequate? .................. 4-3

4.4 Develop Plan/Obtain Data . . . . . . . . . . . . . . . . . . . . 4-5

4.5 Are Commercial Treatability Study Providers Available? . . . . . . . . . . . 4-5

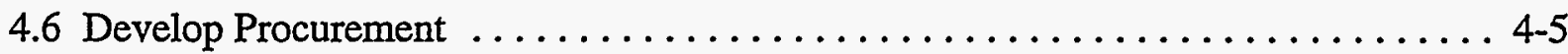

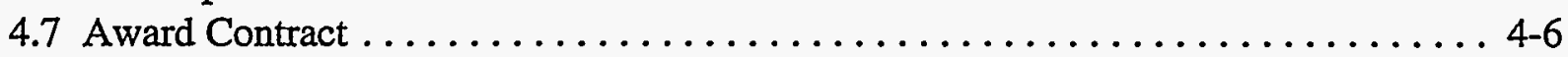

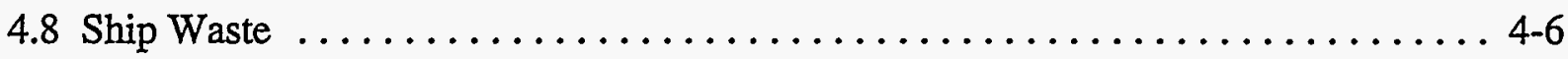



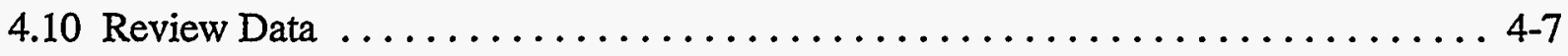


4.11 Disposition of Treatment Residuals

4.12 File Report

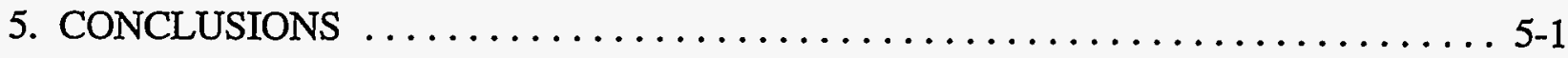

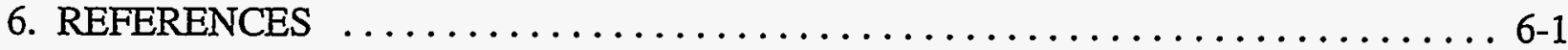

ATTACHMENT 1 Mixed Waste Treatability Study Technology Questionnaire

ATTACHMENT 2 Summaries of Treatability Study Supplier Questionnaire Responses 


\section{LIST OF TABLES}

1-1 Number of U.S. Department of Energy Mixed-Waste Streams Summarized by Volume

$1-2$

1-2 Number of U.S. Department of Energy Mixed-Waste Streams Categorized by RCRARegulated Hazardous Waste Type

2-1 Technology Capabilities of Treatability Study Commercial Providers $\quad 2-2$

2-2 Treatability Study Commercial Providers Capabilities $\quad 2-8$

2-3 Treatability Study Commercial Providers Waste Matrix Capabilities $\quad$ 2-16

3-1 U.S. Department of Transportation Requirements Applicable to Mixed-Waste Treatability Study Samples

4-1 Summary of Three-Stage Data Quality Objectives Development Process

\section{LIST OF FIGURES}

3-1 Treatability Study Regulatory Issues

4-1 Treatability Study Management Issues

4-2 


\section{ACRONYMS/ABBREVIATIONS}

CERCLA Comprehensive Environmental Response, Compensation, and Liability Act

CFR Code of Federal Regulations

DOE

U.S. Department of Energy

DOT

U.S. Department of Transportation

EPA

U.S. Environmental Protection Agency

$\mathrm{kg}$

kilogram(s)

LDR

land disposal restrictions

MTU

mobile treatment unit

MWFA

Mixed Waste Focus Area

NEPA

National Environmental Policy Act

NRC

U.S. Nuclear Regulatory Commission

PCB

polychlorinated biphenyl(s)

ppm

parts per millions

RCRA Resource Conservation and Recovery Act

TSCA Toxic Substances Control Act

VISITT Vendor Information System for Innovative Treatment Technologies (Database) 


\section{EXECUTIVE SUMMARY}

According to the data developed for the proposed site treatment plans, the U.S. Department of Energy (DOE) mixed low-level and mixed transuranic waste inventory was estimated at 181,000 cubic meters comprised of approximately 2,000 waste streams. Many of these streams are unique and require new technologies to facilitate compliance with Resource Conservation and Recovery Act (RCRA) disposal requirements. Because almost all waste streams are unique, a demonstration of the selected technologies is justified. Evaluation of commercially available or innovative technologies in a treatability study is a cost-effective method of providing a demonstration of the technology and supporting decisions on technology selection.

This document provides DOE waste managers with a list of commercial providers with mixed-waste treatability study capabilities and the technologies available at those facilities. The list was compiled by a survey of commercial providers using available databases and a Commerce Business Daily announcement. The survey identified 106 treatment processes offered by 33 commercial providers. The technologies address 21 general treatment categories and include straightforward application of standard technologies (such as pyrolysis) as well as proprietary technologies developed specifically for mixed waste.

DOE mixed-waste treatability studies, conducted in accordance with RCRA, are subject to numerous other federal and state regulations as well as DOE orders. Treatability studies dealing with environmental restoration wastes generated at sites regulated under the Comprehensive Environmental Response, Compensation, and Liability Act (CERCLA) will be subject to CERCLA requirements. If the treatability study involves polychlorinated biphenyls, requirements of the Toxic Substances Control Act will be applicable. If the study involves radionuclides, the facility must have a radioactive materials license from the U.S. Nuclear Regulatory Commission (NRC). Waste samples transported to off-site facilities by common carriers must meet packaging requirements of RCRA, the NRC, and the U.S. Department of Transportation. The DOE is also responsible for complying with the provisions of the National Environmental Policy Act.

Recommendations for the conduct of mixed-waste treatability studies were compiled from several U.S. Environmental Protection Agency guidance manuals, discussions with DOE waste managers who have contracted for off-site treatability studies, and reports generated by the DOE. Twelve separate management steps were identified, starting with a basis question on the need of a treatability study and progressing to identification of data quality objectives and finally to filing the final report. 


\section{INTRODUCTION}

The U.S. Department of Energy (DOE) has established the Mixed Waste Focus Area (MWFA), which represents a national effort to develop and coordinate treatment solutions for mixed waste among all DOE facilities. The hazardous waste component of mixed waste is regulated under the Resource Conservation and Recovery Act (RCRA), while the radioactive component is regulated under the Atomic Energy Act, as implemented by the DOE, making mixed waste one of the most complex types of waste for the DOE to manage. The MWFA has the mission to support technologies that meet the needs of the DOE's waste management efforts to characterize, treat, and dispose of mixed waste being generated and stored throughout the DOE complex. The technologies to be supported must meet all regulatory requirements, provide cost and risk improvements over available technologies, and be acceptable to the public.

The most notable features of the DOE's mixed-waste streams are the wide diversity of waste matrices, volumes, radioactivity levels, and RCRA-regulated hazardous contaminants. Table 1-1 is constructed from data from the proposed site treatment plans developed by each DOE site and submitted to DOE Headquarters. The table shows the number of mixed-waste streams and their corresponding volumes. This table illustrates that the DOE has a relatively small number of large-volume mixed-waste streams and a large number of smallvolume mixed-waste streams. There are 1,033 mixed-waste streams with volumes less than 1 cubic meter; 1,112 mixed-waste streams with volumes between 1 and 1,000 cubic meters; and only 61 mixed-waste streams with volumes exceeding 1,000 cubic meters.

Table 1-2 shows the number of DOE mixed-waste streams with various types of RCRA-regulated hazardous wastes identified by waste matrix type and radioactivity classification (low level, transuranic, and high level). This table shows that the largest number of mixed low-level waste streams are in the following mixed lowlevel waste types:

- Organic liquids containing hazardous organics

- Particulates and sludges containing hazardous metals

- Particulates and sludges containing "other" mixed low-level waste

- Debris and soils/debris containing hazardous metals

- Debris and soils/debris containing "other" mixed low-level waste

- Inherently hazardous waste containing hazardous metals.

The greatest number of mixed transuranic waste streams are composed of particulates/sludges, debris, and debris plus soils with potentially similar distributions of RCRA-regulated hazardous waste types. 
Table 1-1

Number of U.S. Department of Energy Mixed-Waste Streams Summarized by Volume

\begin{tabular}{||l|c|c|c|c|c||}
\hline \multicolumn{1}{|c|}{ WASTE TYPE } & $<1 \mathrm{~m}^{3}$ & $1-10 \mathrm{~m}^{3}$ & $10-1,000 \mathrm{~m}^{3}$ & $>1,000 \mathrm{~m}^{3}$ & $\begin{array}{c}\text { Total } \\
\text { Number }\end{array}$ \\
\hline \hline Mixed Low-Level Waste & 909 & 487 & 392 & 34 & 1,822 \\
\hline Mixed Transuranic Waste & 122 & 102 & 127 & 14 & 365 \\
\hline Mixed High-Level Waste & 2 & 0 & 4 & 13 & 19 \\
\hline TOTAL & 1,033 & 589 & 523 & 61 & 2,206 \\
\hline
\end{tabular}

$m^{3}=$ cubic meters.

Table 1-2

Number of U.S. Department of Energy Mixed-Waste Streams

Categorized by RCRA-Regulated Hazardous Waste Type

\begin{tabular}{|c|c|c|c|c|c|c|c|}
\hline \multirow[b]{2}{*}{ WASTE MATRIX } & \multicolumn{5}{|c|}{ HAZARDOUS WASTE TYPE IN MIXED LOW-LEVEL WASTE } & \multirow[b]{2}{*}{$\begin{array}{c}\text { MIXED } \\
\text { TRUP } \\
\text { WASTE } \\
\end{array}$} & \multirow[b]{2}{*}{$\begin{array}{l}\text { MIXED } \\
\text { HIGH- } \\
\text { LEVEL } \\
\text { WASTE }\end{array}$} \\
\hline & $\begin{array}{c}\text { RCRA Toxicity } \\
\text { Characteristicl } \\
\text { Listed } \\
\text { Organics } \\
\end{array}$ & $\begin{array}{c}\text { RCRA Toxicity } \\
\text { Characteristic } \\
\text { Metals }\end{array}$ & $\begin{array}{l}\text { RCRA- } \\
\text { Regulated } \\
\text { Metals and } \\
\text { Organics } \\
\end{array}$ & $\begin{array}{c}\text { RCRA- } \\
\text { Regulated } \\
\text { Mercury }\end{array}$ & "Other" & & \\
\hline $\begin{array}{l}\text { Aqueous } \\
\text { Liquids/Slumies }\end{array}$ & 1 & 61 & 0 & 1 & 90 & 6 & 12 \\
\hline Organic Liquids & 165 & 0 & 94 & 0 & 11 & 4 & 2 \\
\hline Particulates/Sludges & 81 & 177 & 32 & 12 & 135 & 109 & 4 \\
\hline Soils & 1 & 21 & 0 & 2 & 40 & 0 & 1 \\
\hline $\begin{array}{l}\text { Debris and } \\
\text { Soil/Debris }\end{array}$ & 95 & 231 & 86 & 15 & 153 & 221 & 0 \\
\hline Special Waste & 6 & 23 & 1 & 3 & 58 & 5 & 0 \\
\hline Inherently Hazardous & 1 & 132 & 0 & 11 & 83 & 20 & 0 \\
\hline
\end{tabular}

${ }^{a} T R U=$ Transuranic. 
Treatability studies represent an available vehicle for DOE waste managers to evaluate and demonstrate mixed-waste treatment technologies. The MWFA initiated this survey to facilitate the widest possible use of treatability studies by DOE facilities. The intent of this document is to provide a compilation of commercial treatability study providers and their capabilities for consideration in addressing DOE's small- and largevolume mixed-waste streams.

Chapter 2 presents the results of a survey of commercial mixed-waste treatability study providers with licenses and systems that can accept mixed waste. (University-affiliated providers would be considered commercial providers for the purpose of this survey.) The information is compiled in several formats to facilitate access to companies offering capability in any of 21 mixed-waste treatment technology categories. The MWFA notes that the information compiled in this survey should be regarded as a snapshot in time, as the commercial capability for handling mixed waste is developing and changing at a rapid pace.

The MWFA did not attempt to compile mixed-waste treatability study providers resident within DOE. These sources are relatively well known or can be readily obtained by those in the DOE system, whereas the commercial sources were not widely known. Many DOE facilities have already reported treatability studies and have available systems and regulatory arrangements to conduct treatability studies with surrogates, sitegenerated mixed wastes, or off-site waste samples. The MWFA can provide specific technical contacts on these technologies for those interested.

Chapters 3 and 4 of this document are included for those not familiar with the use of treatability studies for mixed waste. This information is intended as guidance that does not supplant regulator discussions or direct contact with those skilled in treatability study execution.

Chapter 3 provides a summary of regulatory provisions pertaining to treatability studies and the requirements for conducting them. It includes summaries of relevant sections of RCRA, the Toxic Substances Control Act (TSCA), the Comprehensive Environmental Response, Compensation, and Liability Act (CERCLA), U.S. Nuclear Regulatory Commission (NRC) laws, U.S. Department of Transportation (DOT) requirements, DOE Orders, and the National Environmental Policy Act (NEPA).

Chapter 4 is a very brief introduction to the management steps necessary for identifying, specifying, and procuring a treatability study demonstration from a commercial provider. Information in this chapter has been compiled from numerous U.S. Environmental Protection Agency (EPA) guidance documents and published "lessons learned" on treatability study experience at several DOE facilities. This chapter is intended for those unfamiliar with the steps and nuances of managing a successful treatability study. 


\section{TREATABILITY STUDY SURVEY RESULTS}

\subsection{Survey Methodology}

The survey of commercial providers with mixed-waste treatability study capabilities involved identification of candidate firms, contact with the firms to ascertain capability and interest, and compilation of resultant information. Candidate commercial providers were compiled from four sources of information. The EPA's Vendor Information System for Innovative Treatment Technologies (VISITT) database (EPA, 1994) was queried for firms with a background in the treatment of both hazardous and radioactive waste. Secondly, the DOE Tech-Con database, resident at Argonne National Laboratory, was also reviewed. In addition, the Idaho National Engineering Laboratory published their Review of Private Sector Treatment, Storage, and Disposal Capacity for Radioactive Waste in January 1995 (Smith et al., 1995). Finally, Lockheed Martin Idaho Technologies, Inc., published an announcement in the Commerce Business Daily to identify any new sources.

Over 100 commercial providers were identified from these four sources, and a representative from the MWFA contacted all firms by phone to determine whether they had current capability and interest in participating in the survey. Subsequently, the interested firms were sent questionnaires to characterize their capability and history in mixed-waste treatability studies. To identify and confirm each provider's specific capability, a questionnaire was developed that asked for the following:

- Name of facility and facility contact

- Technologies available through that firm

- Waste matrices/hazardous waste types treated by that provider

- Copies of radioactive material licenses (to identify Curie limits for acceptance)

- Relevant limits of their waste acceptance criteria

- Demonstration unit capacity

- Treatability study history

- Lists of publications or technical papers presented on the results of that firm's treatability studies.

The complete questionnaire is included as Attachment 1.

\subsection{Survey Results}

Information from the survey forms has been compiled into a database and presented in several forms in this report. Attachment 2 includes one page summaries of the questionnaire responses by each firm for each technology. Table 2-1 compiles all commercial providers responding with capabilities in each of 21 general treatment technology categories. This table also includes the contact names and telephone numbers. 
Table 2-1

Technology Capabilities of Treatability Study Commercial Providers

\begin{tabular}{|c|c|c|c|c|}
\hline Technology by Category & Company & $\begin{array}{l}\text { Rad Mat } \\
\text { License }\end{array}$ & Contact & Phone \\
\hline $\begin{array}{l}\text { AMALGAMATION } \\
\text { Amalgamation }\end{array}$ & IT Corporation & Yes & Ed Alperin & (423) $690-3211$ \\
\hline $\begin{array}{l}\text { BIODEGRADATION } \\
\text { Air Sparging/Soil Vapor } \\
\text { Extraction }\end{array}$ & $\begin{array}{l}\text { Brown and Root } \\
\text { Environmental }\end{array}$ & Yes & Richard Ninesteel & (412) $921-8746$ \\
\hline $\begin{array}{l}\text { Excavated Soil Biotreatment } \\
\text { (BIOFAST) }\end{array}$ & IT Corporation & Yes & Ed Alperin & (423) 690-3211 \\
\hline Modified Activated Sludge & Maxim Technologies, Inc. & Yes & Robert Bessent & (314) 426-0880 \\
\hline Natural Attenuation & IT Corporation & Yes & Ed Alperin & (423) 690-3211 \\
\hline Research and Development & Radian Corporation & Yes & Rick Strickert & (512) 244-0855 \\
\hline $\begin{array}{l}\text { CHEMICAL OXIDATION } \\
\text { Chemical Photolysis }\end{array}$ & $\begin{array}{l}\text { Institute for Environmental } \\
\text { Health and Safety }\end{array}$ & Yes & Solomon Leung & (208) $236-2524$ \\
\hline Fenton's Reagent Oxidation & $\begin{array}{l}\text { Institute for Environmental } \\
\text { Health and Safety }\end{array}$ & Yes & Solomon Leung & (208) $236-2524$ \\
\hline In-situ Chemical Treatment & $\begin{array}{l}\text { Brown and Root } \\
\text { Environmental }\end{array}$ & Yes & Richard Ninesteel & (412) 921-8746 \\
\hline Research and Development & Radian Corporation & Yes & Rick Strickert & (512) 244-0855 \\
\hline UV Photolysis & IT Corporation & Yes & Ed Alperin & (423) 690-3211 \\
\hline $\begin{array}{l}\text { CONTAINMENT } \\
\text { Fracturing to Increase } \\
\text { Premeability in Bedrock }\end{array}$ & $\begin{array}{l}\text { Brown and Root } \\
\text { Environmental }\end{array}$ & Yes & Richard Ninesteel & (412) $921-8746$ \\
\hline $\begin{array}{l}\text { Pressure Grouting of Soil and } \\
\text { Fractured Bedrock }\end{array}$ & $\begin{array}{l}\text { Brown and Root } \\
\text { Environmental }\end{array}$ & Yes & Richard Ninesteel & (412) $921-8746$ \\
\hline Soil Saw Barrier System & $\begin{array}{l}\text { Brown and Root } \\
\text { Environmental }\end{array}$ & Yes & Richard Ninesteel & (412) $921-8746$ \\
\hline DEACTIVATION & & & & \\
\hline $\begin{array}{l}\text { Compressed Gases } \\
\text { Deactivation }\end{array}$ & $\begin{array}{l}\text { Earth Resources Corporation } \\
\text { IT Corporation }\end{array}$ & $\begin{array}{l}\text { No } \\
\text { Yes }\end{array}$ & $\begin{array}{l}\text { Michael Miller } \\
\text { Ed Alperin }\end{array}$ & $\begin{array}{l}(407) 877-0877 \\
(423) 690-3211\end{array}$ \\
\hline $\begin{array}{l}\text { DEBRIS WASHING } \\
\text { Debris Decontamination }\end{array}$ & IT Corporation & Yes & Ed Alperin & (423) 690-3211 \\
\hline $\begin{array}{l}\text { EXTRACTION } \\
\text { Electrochemical Ion Exchange } \\
(E X X)\end{array}$ & Selentec & No & Michael Dunn & (770) $640-7059$ \\
\hline $\begin{array}{l}\text { INCINERATION } \\
\text { Disposal of Liquid Organics }\end{array}$ & $\begin{array}{l}\text { Perma-Fix Environmental } \\
\text { Services }\end{array}$ & Yes & $\begin{array}{l}\text { Bernhardt (Ben) } \\
\text { Warren }\end{array}$ & (352) $395-1352$ \\
\hline Fluid-Bed Calcination & $\begin{array}{l}\text { Energy and Environmental } \\
\text { Research Center }\end{array}$ & No & Edward Steadman & (701) 777-5157 \\
\hline Incineration & IT Corporation & Yes & Ed Alperin & (423) $690-3211$ \\
\hline $\begin{array}{l}\text { Industrial boiler used for energy } \\
\text { recovery }\end{array}$ & $\begin{array}{l}\text { Chemical Waste } \\
\text { Management, Inc. }\end{array}$ & Yes & Richard Dabolt & (423) $376-0084$ \\
\hline
\end{tabular}


Table 2-1 (Continued)

\section{Technology Capabilities of Treatability Study Commercial Providers}

\begin{tabular}{|c|c|c|c|c|}
\hline Technology by Category & Company & $\begin{array}{l}\text { Rad Mat } \\
\text { License }\end{array}$ & Contact & Phone \\
\hline LSV Processing & $\begin{array}{l}\text { Perma-Fix Environmental } \\
\text { Services }\end{array}$ & Yes & $\begin{array}{l}\text { Bernhardt (Ben) } \\
\text { Warren }\end{array}$ & (352) 395-1352 \\
\hline Pyrolysis & $\begin{array}{l}\text { Energy and Environmental } \\
\text { Research Center }\end{array}$ & No & Edward Steadman & (701) 777-5157 \\
\hline \multicolumn{5}{|l|}{ MACROENCAPSULATION } \\
\hline Macroencapsulation & Envirocare of Utah, Inc. & Yes & Kevin Fuller & (801) 532-1330 \\
\hline Macroencapsulation & IT Corporation & Yes & Ed Alperin & (423) 690-3211 \\
\hline \multicolumn{5}{|l|}{ ORGANIC DESTRUCTION } \\
\hline $\begin{array}{l}\text { Base Catalyzed Decomposition } \\
\text { (BCD) }\end{array}$ & IT Corporation & Yes & Ed Alperin & (423) 690-3211 \\
\hline Chemical Dehalogenation & IT Corporation & Yes & Ed Alperin & (423) $690-3211$ \\
\hline DECHLOR@ & WMXX Technologies Inc. & Yes & $\begin{array}{l}\text { Edward } \\
\text { Wannemacher }\end{array}$ & (864) 646-2413 \\
\hline $\begin{array}{l}\mathrm{H} 2 \mathrm{O} 2 / \text { Ozone Treatment of } \\
\text { Liquid Waste }\end{array}$ & Maxim Technologies, Inc. & Yes & Robert Bessent & (314) $426-0880$ \\
\hline $\begin{array}{l}\text { Incineration/Combustion/Calcini } \\
\text { ng/Roasting }\end{array}$ & Hazen Research Inc. & Yes & Rodney Hodgson & (303) 279-4501 \\
\hline Molten Salt Oxidation & WMX Technologies Inc. & Yes & $\begin{array}{l}\text { Edward } \\
\text { Wannemacher }\end{array}$ & (864) 646-2413 \\
\hline $\begin{array}{l}\text { Permeable treatment bed } \\
\text { installation }\end{array}$ & $\begin{array}{l}\text { Brown and Root } \\
\text { Environmental }\end{array}$ & Yes & Richard Ninesteel & (412) $921-8746$ \\
\hline PO*WW*ER@ & WMX Technologies Inc. & Yes & $\begin{array}{l}\text { Edward } \\
\text { Wannemacher }\end{array}$ & (864) 646-2413 \\
\hline Steam Reforming & $\begin{array}{l}\text { Scientific Ecology Group, } \\
\text { Inc. }\end{array}$ & Yes & Tom Snyder & (423) $376-8321$ \\
\hline $\begin{array}{l}\text { UV Photolysis/Chemical } \\
\text { Oxidation }\end{array}$ & IT Corporation & Yes & Ed Alperin & (423) 690-3211 \\
\hline $\begin{array}{l}\text { PHYSICAL SEPARATION } \\
\text { Segmented Gate System }\end{array}$ & Thermo NUtech & No & Jeff Brown & (423) 481-0683 \\
\hline RECOVERY OF METALS & & & & \\
\hline Activated Carbon & $\begin{array}{l}\text { Energy and Environmental } \\
\text { Research Center }\end{array}$ & No & Edward Steadman & (701) 777-5157 \\
\hline Centrifugal Membrane Filtration & $\begin{array}{l}\text { Energy and Environmental } \\
\text { Research Center }\end{array}$ & No & Edward Steadman & (701) 777-5157 \\
\hline CHEMICTM & $\begin{array}{l}\text { Atomic Energy of Canada } \\
\text { Limited (AECL) }\end{array}$ & Yes & Leo Buckley & (613) 584-8811 \\
\hline Chemical Leaching & Hazen Research Inc. & Yes & Rodney Hodgson & (303) 279-4501 \\
\hline Diphonix Ion Exchange & Eichrom Industries, Inc. & No & James Harvey & (708) 963-0320 \\
\hline Electro-Chemical Separation & IT Corporation & Yes & Ed Alperin & (423) $690-3211$ \\
\hline Freeze Separation & WMX Technologies Inc. & Yes & $\begin{array}{l}\text { Edward } \\
\text { Wannemacher }\end{array}$ & (864) $646-2413$ \\
\hline MAG*SEP & Selentec & No & Michael Dunn & (770) 640-7059 \\
\hline Precipitation & IT Corporation & Yes & Ed Alperin & (423) $690-3211$ \\
\hline
\end{tabular}


Table 2-1 (Continued)

Technology Capabilities of Treatability Study Commercial Providers

\begin{tabular}{|c|c|c|c|c|}
\hline Technology by Category & Company & $\begin{array}{l}\text { Rad Mat } \\
\text { License }\end{array}$ & Contact & Phone \\
\hline $\begin{array}{l}\text { Quantum - Catalytic Extraction } \\
\text { Processing }\end{array}$ & $\begin{array}{l}\text { M4 Environmental } \\
\text { Management Inc. }\end{array}$ & Yes & Robert Sameski & (423) 220-5017 \\
\hline Reverse Osmosis & IT Corporation & Yes & Ed Alperin & (423) 690-3211 \\
\hline Solvent Extraction & Hazen Research Inc. & Yes & Rodney Hodgson & (303) 279-4501 \\
\hline \multicolumn{5}{|l|}{ RECOVERY OF ORGANICS } \\
\hline Adsorption Media & IT Corporation & Yes & Ed Alperin & (423) $690-3211$ \\
\hline $\begin{array}{l}\text { Chemical Extraction, with or } \\
\text { without chelants }\end{array}$ & IT Corporation & Yes & Ed Alperin & (423) 690-3211 \\
\hline $\begin{array}{l}\text { Liquified Gas Solvent Extraction } \\
\text { (LG-SX) }\end{array}$ & CF Systems & Access & Michael Finton & (216) $523-5286$ \\
\hline Thermal Desorption & Hazen Research Inc. & Yes & Rodney Hodgson & (303) 279-4501 \\
\hline VAC*TRAXX $^{\text {TM }}$ & WMX Technologies Inc. & Yes & $\begin{array}{l}\text { Edward } \\
\text { Wannemacher }\end{array}$ & (864) 646-2413 \\
\hline \multicolumn{5}{|l|}{ RETORTING FOR MERCURY } \\
\hline $\begin{array}{l}\text { Low Temperature Thermal } \\
\text { Desorption }\end{array}$ & IT Corporation & Yes & Ed Alperin & (423) 690-3211 \\
\hline Retorting for Mercury & Hazen Research Inc. & Yes & Rodney Hodgson & (303) $279-4501$ \\
\hline Retorting for Mercury & Nuclear Fuel Services, Inc. & Yes & R. David Wise & (423) $743-1795$ \\
\hline \multicolumn{5}{|l|}{ SOIL WASHING } \\
\hline Acid Extraction of Soil/Sediment & Maxim Technologies, Inc. & Yes & Robert Bessent & (314) $426-0880$ \\
\hline $\mathrm{ACT} * \mathrm{DE}^{*} \mathrm{CON}$ & Selentec & No & Michael Dunn & (770) 640-7059 \\
\hline $\begin{array}{l}\text { Materials Handling/Soil } \\
\text { Washing/Gravity Separation }\end{array}$ & Hazen Research Inc. & Yes & Rodney Hodgson & (303) 279-4501 \\
\hline Soil Screening/Washing & $\begin{array}{l}\text { Babcock \& Wilcox, A } \\
\text { McDermott Company }\end{array}$ & & Nicholas Mravich & (330) 829-7662 \\
\hline Soil Washing & $\begin{array}{l}\text { Alternative Remedial } \\
\text { Technologies }\end{array}$ & No & Michael Mann & (813) 264-3571 \\
\hline Soil Washing & Nuclear Fuel Services, Inc. & Yes & R. David Wise & (423) 743-1795 \\
\hline Soil Washing & $\begin{array}{l}\text { Westinghouse Science and } \\
\text { Technology Center }\end{array}$ & Yes & Nancy Ulerich & (412) $256-2198$ \\
\hline Soil Washing/Flushing & IT Corporation & Yes & Ed Alperin & (423) $690-3211$ \\
\hline SOIL*EXTM & WMX Technologies Inc. & Yes & $\begin{array}{l}\text { Edward } \\
\text { Wannemacher }\end{array}$ & (864) 646-2413 \\
\hline $\begin{array}{l}\text { Terra-Kleen Solvent Extraction } \\
\text { System }\end{array}$ & $\begin{array}{l}\text { Terra-Kleen Response } \\
\text { Group, Inc. }\end{array}$ & & Alan Cash & (619) 558-8762 \\
\hline TVIES & TVIES & No & Myron Kuhlman & (713) $447-5544$ \\
\hline \multicolumn{5}{|l|}{ STABUIZATION } \\
\hline Aquaset/Petroset & Fluid Tech, Inc. & Yes & Thomas Murarik & (702) 871-1884 \\
\hline Auger Mixing System & $\begin{array}{l}\text { Brown and Root } \\
\text { Environmental }\end{array}$ & Yes & Richard Ninesteel & (412) $921-8746$ \\
\hline Cement Stabilization & $\begin{array}{l}\text { Scientific Ecology Group, } \\
\text { Inc. }\end{array}$ & Yes & Marty Brownstein & (423) $376-8321$ \\
\hline CHEM MATRIX & WMX Technologies Inc. & Yes & $\begin{array}{l}\text { Edward } \\
\text { Wannemacher }\end{array}$ & (864) 646-2413 \\
\hline
\end{tabular}


Table 2-1 (Continued)

\section{Technology Capabilities of Treatability Study Commercial Providers}

\begin{tabular}{|c|c|c|c|c|}
\hline Technology by Category & Company & $\begin{array}{l}\text { Rad Mat } \\
\text { License }\end{array}$ & Contact & Phone \\
\hline Chemical Stabilization & Nuclear Fuel Services, Inc. & Yes & R. David Wise & (423) 743-1795 \\
\hline $\begin{array}{l}\text { High Shear Waste } \\
\text { Immobilization }\end{array}$ & $\begin{array}{l}\text { Westinghouse Science and } \\
\text { Technology Center }\end{array}$ & Yes & Nancy Ulerich & (412) $256-2198$ \\
\hline Jet Grouting & $\begin{array}{l}\text { Brown and Root } \\
\text { Environmental }\end{array}$ & Yes & Richard Ninesteel & (412) $921-8746$ \\
\hline $\begin{array}{l}\text { Magnesium Oxide Cement } \\
\text { Stabilization }\end{array}$ & $\begin{array}{l}\text { Scientific Ecology Group, } \\
\text { Inc. }\end{array}$ & Yes & Marty Brownstein & (423) $376-8321$ \\
\hline Non-Thermal Stabilization & Envirocare of Utah, Inc. & Yes & Kevin Fuller & (801) 532-1330 \\
\hline Perma-Fix Process & $\begin{array}{l}\text { Perma-Fix Environmental } \\
\text { Services }\end{array}$ & Yes & $\begin{array}{l}\text { Bernhardt (Ben) } \\
\text { Warren }\end{array}$ & (352) $395-1352$ \\
\hline $\begin{array}{l}\text { Phoenix Ash Technology (TIDE } \\
\text { Bricks) }\end{array}$ & WMX Technologies Inc. & Yes & $\begin{array}{l}\text { Edward } \\
\text { Wannemacher }\end{array}$ & (864) 646-2413 \\
\hline Polyethylene Stabilization & $\begin{array}{l}\text { Scientific Ecology Group, } \\
\text { Inc. }\end{array}$ & Yes & Marty Brownstein & (423) $376-8321$ \\
\hline Research and Development & $\begin{array}{l}\text { Perma-Fix Environmental } \\
\text { Services }\end{array}$ & Yes & $\begin{array}{l}\text { Bernhardt (Ben) } \\
\text { Warren }\end{array}$ & (352) 395-1352 \\
\hline Research and Development & Radian Corporation & Yes & Rick Strickert & (512) 244-0855 \\
\hline Soil Rake & $\begin{array}{l}\text { Brown and Root } \\
\text { Environmental }\end{array}$ & Yes & Richard Ninesteel & (412) $921-8746$ \\
\hline Stabilization/ Solidification & IT Corporation & Yes & Ed Alperin & (423) $690-3211$ \\
\hline Superset & $\begin{array}{l}\text { Carlo Environmental } \\
\text { Technologies, Inc. }\end{array}$ & No & Keith Flemingloss & (810) 468-9580 \\
\hline $\begin{array}{l}\text { Thermoplastic Epoxy } \\
\text { Stabilization }\end{array}$ & $\begin{array}{l}\text { Scientific Ecology Group, } \\
\text { Inc. }\end{array}$ & Yes & Marty Brownstein & (423) $376-8321$ \\
\hline Thermoset Epoxy Stabilization & $\begin{array}{l}\text { Scientific Ecology Group, } \\
\text { Inc. }\end{array}$ & Yes & Marty Brownstein & (423) $376-8321$ \\
\hline Vinyl Ester Stabilization & $\begin{array}{l}\text { Scientific Ecology Group, } \\
\text { Inc. }\end{array}$ & Yes & Marty Brownstein & (423) $376-8321$ \\
\hline \multicolumn{5}{|l|}{ STEAM STRIPPING } \\
\hline Organic Phase Transfer & Maxim Technologies, Inc. & Yes & Robert Bessent & (314) $426-0880$ \\
\hline Steam/Air Stripping & IT Corporation & Yes & Ed Alperin & (423) $690-3211$ \\
\hline \multicolumn{5}{|l|}{ THERMAL DESORPTION } \\
\hline Terrachem IRV-100 & McLaren-Hart Inc. & & Jeff Oham & (704) 587-0003 \\
\hline \multicolumn{5}{|l|}{ VITRIFICATION } \\
\hline Cold Crucible Vitrification & WMXX Technologies Inc. & Yes & $\begin{array}{l}\text { Edward } \\
\text { Wannemacher }\end{array}$ & (864) 646-2413 \\
\hline Cyclone Furnace Vitrification & $\begin{array}{l}\text { Babcock \& Wilcox, A } \\
\text { McDermott Company }\end{array}$ & & Michael Holmes & (330) 829-7662 \\
\hline DC Graphite Arc Melter & Electro-Pyrolysis, Inc. & Access & J. Kenneth Wittle & (610) 687-9070 \\
\hline Enviroglass & Vectra & & Paul Denault & (803) 781-0426 \\
\hline In Situ Vitrification (ISV) & Geosafe Corporation & Access & Matthew Haass & (509) $375-0710$ \\
\hline Joule-Heated Melter & $\begin{array}{l}\text { Scientific Ecology Group, } \\
\text { Inc. }\end{array}$ & Yes & Tom Snyder & (423) $376-8321$ \\
\hline
\end{tabular}


Table 2-1 (Continued)

Technology Capabilities of Treatability Study Commercial Providers

\begin{tabular}{|c|c|c|c|c|}
\hline Technology by Category & Company & $\begin{array}{l}\text { Rad Mat } \\
\text { License }\end{array}$ & Contact & Phone \\
\hline Joule-Heated Melters & WMX Technologies Inc. & Yes & $\begin{array}{l}\text { Edward } \\
\text { Wannemacher }\end{array}$ & (864) $646-2413$ \\
\hline Plasma & $\begin{array}{l}\text { Westinghouse Science and } \\
\text { Technology Center }\end{array}$ & Yes & Nancy Ulerich & (412) $256-2198$ \\
\hline Plasma Centrifugal Furnace & $\begin{array}{l}\text { MSE Technology } \\
\text { Applications, Inc. }\end{array}$ & No & Jeff Ruffner & (406) 494-7412 \\
\hline Plasma Hearth Process & $\begin{array}{l}\text { Science Applications } \\
\text { International Corporation }\end{array}$ & No & Gary Leatherman & (208) 528-2179 \\
\hline Plasma-Driven Reactor & $\begin{array}{l}\text { Scientific Ecology Group, } \\
\text { Inc. }\end{array}$ & Yes & Tom Snyder & (423) $376-8321$ \\
\hline Vitrification & IT Corporation & Yes & Ed Alperin & (423) 690-3211 \\
\hline $\begin{array}{l}\text { WASTE SEPARATION } \\
\text { Bulking, Profiling for Disposal } \\
\text { or Incineration }\end{array}$ & $\begin{array}{l}\text { Perma-Fix Environmental } \\
\text { Services }\end{array}$ & Yes & $\begin{array}{l}\text { Bernhardt (Ben) } \\
\text { Warren }\end{array}$ & (352) $395-1352$ \\
\hline WET OXIDATION & & & & \\
\hline Hydrothermal Boiler & $\begin{array}{l}\text { Energy and Environmental } \\
\text { Research Center }\end{array}$ & No & Edward Steadman & (701) 777-5157 \\
\hline Wet Air Oxidation & IT Corporation & Yes & Ed Alperin & (423) 690-3211 \\
\hline
\end{tabular}


Note that Table 2-1 includes an indication of whether the commercial providers possess radioactive materials licenses. This is a key item because technology demonstrations under RCRA require only notification of the cognizant agency (see Chapter 3 for details). Therefore, the radioactive material license is a key indicator of the ability to accept mixed-waste treatability study samples. Column 3 of Table 2-1 indicates a few commercial providers with "access" to licensed facilities. In this case, the respondents indicated that they do not have their own licensed facilities, but they have arrangements with other commercial or government facilities with appropriate licenses and permits. An alternative by at least one commercial provider is that it has agreements with DOE sites for radioactive material treatment demonstrations.

A second tabulation, Table 2-2, compiles all treatment technologies available from each commercial provider tabulated by company. Aside from the contact information, the table includes the level of technology maturity (discussed in the next paragraph), the type of waste acceptable at the facility or suitable for the technology, and the regulatory status of resultant treatment residues. (This latter information is important, as will be described in Chapter 4.) The residues from treatability studies undergo a hazardous waste determination, and the customers must plan for whether the treatment residues are radioactive waste, mixed waste meeting disposal requirements, or mixed waste requiring further treatment. A third table, Table 2-3, provides wastematrix information for each technology offered by each commercial provider tabulated by company and technology.

In Table 2-2, the maturity level of the technologies is given as bench scale, pilot scale, or commercial scale. In general, the level was determined by the commercial provider. In making the assignment, the term "bench scale" describes a process demonstrated on a small scale, and usually only the single-unit operation was demonstrated. Capacities of liters or liters per day is common for bench scale apparatus. The term "pilot scale" refers to a process demonstrated on an integrated pilot plant scale where the scale of operation is in the gallons-per-day range. Pilot plants usually include some degree of automatic control. "Commercial scale" processes have been operated using purchased commercial equipment or by a commercial service supplier. This scale typically involves drum-size quantities of waste or more.

A review of the information in Tables 2-1 through 2-3 and the accompanying commercial provider/technology questionnaire response summaries in Attachment 2 reveals several groupings of survey respondents:

- Commercial technology suppliers with a few technologies who typically do not have available mixedwaste treatability study facilities.

- Commercial technology suppliers with licenses for mixed-waste treatability studies and many technologies available. 
Table 2-2

Treatability Study Commercial Providers Capabilities

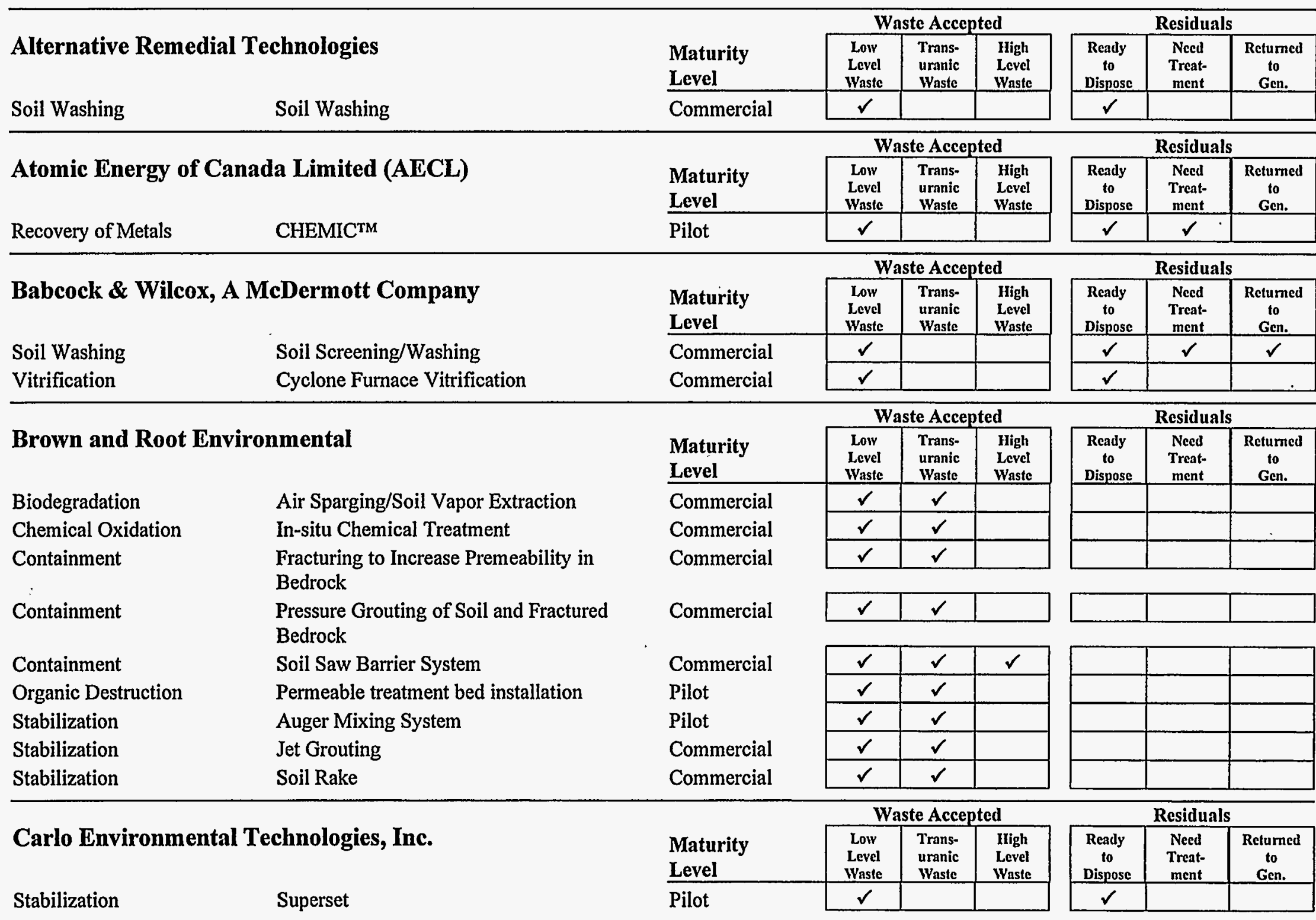


Table 2-2 (Continued)

Treatability Study Commercial Providers Capabilities

\begin{tabular}{|c|c|c|c|c|c|c|c|c|}
\hline \multirow[b]{2}{*}{ CF Systems } & \multirow{2}{*}{ 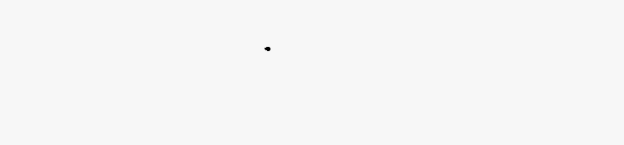 } & \multirow[b]{2}{*}{$\begin{array}{l}\text { Maturity } \\
\text { Level }\end{array}$} & \multicolumn{3}{|c|}{ Waste Accepted } & \multicolumn{3}{|c|}{ Residuals } \\
\hline & & & $\begin{array}{l}\text { Low } \\
\text { Level } \\
\text { Waste } \\
\end{array}$ & $\begin{array}{l}\text { Trans- } \\
\text { uranic } \\
\text { Waste } \\
\end{array}$ & $\begin{array}{l}\text { High } \\
\text { Level } \\
\text { Waste. }\end{array}$ & $\begin{array}{c}\text { Ready } \\
\text { to } \\
\text { Dispose } \\
\end{array}$ & $\begin{array}{l}\text { Need } \\
\text { Treat- } \\
\text { ment }\end{array}$ & $\begin{array}{l}\text { Returned } \\
\text { to } \\
\text { Gen. } \\
\end{array}$ \\
\hline Recovery of Organics & Liquified Gas Solvent Extraction & Commercial & $\checkmark$ & $\checkmark$ & $\checkmark$ & $\checkmark$ & & \\
\hline & (LG-SX) & & & & & & & \\
\hline \multirow{2}{*}{\multicolumn{2}{|c|}{ Chemical Waste Management, Inc. }} & & \multicolumn{3}{|c|}{ Waste Accepted } & \multicolumn{3}{|c|}{ Residuals } \\
\hline & & $\begin{array}{l}\text { Maturity } \\
\text { Level }\end{array}$ & $\begin{array}{l}\text { Low } \\
\text { Level } \\
\text { Waste } \\
\end{array}$ & $\begin{array}{l}\text { Trans- } \\
\text { uranic } \\
\text { Waste }\end{array}$ & $\begin{array}{l}\text { High } \\
\text { Levcl } \\
\text { Wastc }\end{array}$ & \begin{tabular}{|c|} 
Ready \\
to \\
Disposc \\
\end{tabular} & $\begin{array}{l}\text { Need } \\
\text { Treat- } \\
\text { ment }\end{array}$ & $\begin{array}{c}\text { Retumed } \\
\text { to } \\
\text { Gen. }\end{array}$ \\
\hline Incineration & Industrial boiler used for energy recovery & Commercial & $\checkmark$ & $\checkmark$ & & $\checkmark$ & $\checkmark$ & \\
\hline \multirow{2}{*}{\multicolumn{2}{|c|}{ Earth Resources Corporation }} & & \multicolumn{3}{|c|}{ Waste Accepted } & \multicolumn{3}{|c|}{ Residuals } \\
\hline & & $\begin{array}{l}\text { Maturity } \\
\text { Level } \\
\end{array}$ & $\begin{array}{l}\text { Low } \\
\text { Level } \\
\text { Waste } \\
\end{array}$ & $\begin{array}{l}\text { Trans- } \\
\text { uranic } \\
\text { Waste } \\
\end{array}$ & $\begin{array}{l}\text { High } \\
\text { Level } \\
\text { Waste } \\
\end{array}$ & $\begin{array}{c}\text { Ready } \\
\text { to } \\
\text { Dispose } \\
\end{array}$ & $\begin{array}{l}\text { Need } \\
\text { Treat- } \\
\text { ment }\end{array}$ & $\begin{array}{c}\text { Retumed } \\
\text { to } \\
\text { Gen. }\end{array}$ \\
\hline Deactivation & Compressed Gases & Commercial & $\checkmark$ & & & $\checkmark$ & $\checkmark$ & \\
\hline \multicolumn{2}{|c|}{ Eichrom Industries, Inc. } & & \multicolumn{3}{|c|}{ Waste Accepted } & \multicolumn{3}{|c|}{ Residuals } \\
\hline Eichrom Industrie & & $\begin{array}{l}\text { Maturity } \\
\text { Level } \\
\end{array}$ & $\begin{array}{l}\text { Low } \\
\text { Level } \\
\text { Waste } \\
\end{array}$ & $\begin{array}{l}\text { Trans- } \\
\text { uranic } \\
\text { Waste }\end{array}$ & $\begin{array}{l}\text { High } \\
\text { Level } \\
\text { Waste } \\
\end{array}$ & $\begin{array}{c}\text { Ready } \\
\text { to } \\
\text { Dispose } \\
\end{array}$ & $\begin{array}{c}\text { Need } \\
\text { Treat- } \\
\text { ment }\end{array}$ & $\begin{array}{c}\text { Returned } \\
\text { to } \\
\text { Gen. }\end{array}$ \\
\hline Recovery of Metals & Diphonix Ion Exchange & Commercial & $\checkmark$ & & & $\checkmark$ & & \\
\hline \multirow{2}{*}{\multicolumn{2}{|c|}{ Electro-Pyrolysis, Inc. }} & & \multicolumn{3}{|c|}{ Waste Accepted } & \multicolumn{3}{|c|}{ Residuals } \\
\hline & & $\begin{array}{l}\text { Maturity } \\
\text { Level }\end{array}$ & $\begin{array}{l}\text { Low } \\
\text { Level } \\
\text { Waste } \\
\end{array}$ & $\begin{array}{l}\text { Trans- } \\
\text { uranic } \\
\text { Waste } \\
\end{array}$ & $\begin{array}{l}\text { High } \\
\text { Leval } \\
\text { Waste } \\
\end{array}$ & $\begin{array}{c}\text { Ready } \\
\text { to } \\
\text { Dispose } \\
\end{array}$ & $\begin{array}{l}\text { Need } \\
\text { Treat- } \\
\text { ment } \\
\end{array}$ & $\begin{array}{c}\text { Retumed } \\
\text { to } \\
\text { Gen. } \\
\end{array}$ \\
\hline Vitrification & DC Graphite Arc Melter & Pilot & & & & $\checkmark$ & & $\checkmark$ \\
\hline \multirow{2}{*}{\multicolumn{2}{|c|}{ Energy and Environmental Research Center }} & & \multicolumn{3}{|c|}{ Waste Accepted } & \multicolumn{3}{|c|}{ Residuals } \\
\hline & & $\begin{array}{l}\text { Maturity } \\
\text { Level } \\
\end{array}$ & $\begin{array}{l}\text { Low } \\
\text { Level } \\
\text { Waste } \\
\end{array}$ & $\begin{array}{l}\begin{array}{l}\text { Trans- } \\
\text { uranic } \\
\text { Waste } \\
\end{array} \\
\end{array}$ & $\begin{array}{l}\text { High } \\
\text { Level } \\
\text { Waste } \\
\end{array}$ & $\begin{array}{c}\text { Ready } \\
\text { to } \\
\text { Dispose }\end{array}$ & $\begin{array}{c}\begin{array}{c}\text { Necd } \\
\text { Treat- } \\
\text { ment }\end{array} \\
\end{array}$ & $\begin{array}{c}\text { Returned } \\
\text { to } \\
\text { Gen. }\end{array}$ \\
\hline \multirow{5}{*}{$\begin{array}{l}\text { Incineration } \\
\text { Incineration } \\
\text { Recovery of Metals } \\
\text { Recovery of Metals } \\
\text { Wet Oxidation }\end{array}$} & \multirow{5}{*}{$\begin{array}{l}\text { Fluid-Bed Calcination } \\
\text { Pyrolysis } \\
\text { Activated Carbon } \\
\text { Centrifugal Membrane Filtration } \\
\text { Hydrothermal Boiler }\end{array}$} & \multirow{5}{*}{$\begin{array}{l}\text { Bench } \\
\text { Pilot } \\
\text { Pilot } \\
\text { Commercial } \\
\text { R \& D }\end{array}$} & & & & $\checkmark$ & & \\
\hline & & & & & & & $\checkmark$ & \\
\hline & & & $\checkmark$ & & & & & \\
\hline & & & $\checkmark$ & $\checkmark$ & $\checkmark$ & $\checkmark$ & $\checkmark$ & $\checkmark$ \\
\hline & & & $\checkmark$ & & & & $\checkmark$ & \\
\hline
\end{tabular}


Table 2-2 (Continued)

Treatability Study Commercial Providers Capabilities

\begin{tabular}{|c|c|c|c|c|c|c|c|c|}
\hline \multirow{2}{*}{\multicolumn{2}{|c|}{ Envirocare of Utah, Inc. }} & \multirow[b]{2}{*}{$\begin{array}{l}\text { Maturity } \\
\text { Level }\end{array}$} & \multicolumn{3}{|c|}{ Waste Accepted } & \multicolumn{3}{|c|}{ Residuals } \\
\hline & & & $\begin{array}{l}\text { Low } \\
\text { Level } \\
\text { Waste }\end{array}$ & $\begin{array}{l}\text { Trans: } \\
\text { uranic } \\
\text { Waste }\end{array}$ & $\begin{array}{l}\text { High } \\
\text { Level } \\
\text { Waste }\end{array}$ & $\begin{array}{c}\text { Ready } \\
\text { to } \\
\text { Dispose }\end{array}$ & $\begin{array}{c}\text { Need } \\
\text { Treat- } \\
\text { ment }\end{array}$ & $\begin{array}{c}\text { Returned } \\
\text { to } \\
\text { Gen. }\end{array}$ \\
\hline \multirow{2}{*}{$\begin{array}{l}\text { Macroencapsulation } \\
\text { Stabilization }\end{array}$} & \multirow{2}{*}{$\begin{array}{l}\text { Macroencapsulation } \\
\text { Non-Thermal Stabilization }\end{array}$} & \multirow{2}{*}{$\begin{array}{l}\text { Commercial } \\
\text { Commercial }\end{array}$} & $\checkmark$ & $\checkmark$ & & $\checkmark$ & & \\
\hline & & & $\checkmark$ & $\checkmark$ & & $\checkmark$ & & \\
\hline \multirow{2}{*}{\multicolumn{2}{|c|}{ Fluid Tech, Inc. }} & & \multicolumn{3}{|c|}{ Waste Accented } & \multicolumn{3}{|c|}{ Residuals } \\
\hline & & $\begin{array}{l}\text { Maturity } \\
\text { Level } \\
\end{array}$ & $\begin{array}{l}\text { Low } \\
\text { Level } \\
\text { Waste } \\
\end{array}$ & $\begin{array}{l}\text { Trans- } \\
\text { uranic } \\
\text { Waste } \\
\end{array}$ & $\begin{array}{l}\text { High } \\
\text { Level } \\
\text { Waste } \\
\end{array}$ & $\begin{array}{c}\text { Ready } \\
\text { to } \\
\text { Dispose }\end{array}$ & $\begin{array}{c}\text { Need } \\
\text { Treant- } \\
\text { ment }\end{array}$ & $\begin{array}{c}\text { Returned } \\
\text { to } \\
\text { Gen. } \\
\end{array}$ \\
\hline Stabilization & Aquaset/Petroset & Commercial & $\checkmark$ & $\checkmark$ & & & $\checkmark$ & \\
\hline \multirow{2}{*}{\multicolumn{2}{|c|}{ Geosafe Corporation }} & & \multicolumn{3}{|c|}{ Waste Accepted } & \multicolumn{3}{|c|}{ Residuals } \\
\hline & & $\begin{array}{l}\text { Maturity } \\
\text { Level } \\
\end{array}$ & $\begin{array}{l}\text { Low } \\
\text { Level } \\
\text { Waste }\end{array}$ & $\begin{array}{l}\text { Trans- } \\
\text { uranic } \\
\text { Waste }\end{array}$ & $\begin{array}{l}\text { High } \\
\text { Level } \\
\text { Wastc }\end{array}$ & $\begin{array}{c}\text { Rendy } \\
\text { to } \\
\text { Dispose }\end{array}$ & $\begin{array}{c}\text { Need } \\
\text { Treat- } \\
\text { ment }\end{array}$ & $\begin{array}{c}\text { Retumed } \\
\text { to } \\
\text { Gen. }\end{array}$ \\
\hline Vitrification & In Situ Vitrification (ISV) & Commercial & $\checkmark$ & $\checkmark$ & & $\checkmark$ & & \\
\hline \multirow{2}{*}{\multicolumn{2}{|c|}{ Hazen Research Inc. }} & & \multicolumn{3}{|c|}{ Waste Accepted } & \multicolumn{3}{|c|}{ Residuals } \\
\hline & & $\begin{array}{l}\text { Maturity } \\
\text { Level } \\
\end{array}$ & $\begin{array}{l}\text { Low } \\
\text { Level } \\
\text { Waste }\end{array}$ & $\begin{array}{l}\text { Trans- } \\
\text { uranic } \\
\text { Waste }\end{array}$ & $\begin{array}{l}\text { High } \\
\text { Levcl } \\
\text { Waste } \\
\end{array}$ & $\begin{array}{c}\text { Ready } \\
\text { to } \\
\text { Dispose }\end{array}$ & $\begin{array}{c}\text { Need } \\
\text { Treat- } \\
\text { ment }\end{array}$ & $\begin{array}{c}\text { Retumed } \\
\text { to } \\
\text { Gen. }\end{array}$ \\
\hline \multirow{2}{*}{ Organic Destruction } & \multirow{2}{*}{$\begin{array}{l}\text { Incineration/Combustion/Calcining/Roastin } \\
\mathrm{g}\end{array}$} & \multirow[t]{2}{*}{$\overline{\text { Pilot }}$} & \multirow[t]{2}{*}{$\checkmark$} & Waste & & \multirow[t]{2}{*}{$\checkmark$} & \multirow[t]{2}{*}{$\checkmark$} & \multirow[t]{2}{*}{$\checkmark$} \\
\hline & & & & & & & & \\
\hline \multirow{6}{*}{$\begin{array}{l}\text { Recovery of Metals } \\
\text { Recovery of Metals } \\
\text { Recovery of Organics } \\
\text { Retorting for Mercury } \\
\text { Soil Washing }\end{array}$} & Chemical Leaching & \multirow{6}{*}{$\begin{array}{l}\text { Pilot } \\
\text { Pilot } \\
\text { Pilot } \\
\text { Commercial } \\
\text { Pilot }\end{array}$} & \multicolumn{2}{|l|}{$\checkmark$} & & $\checkmark$ & $\checkmark$ & $\checkmark$ \\
\hline & Solvent Extraction & & $\checkmark$ & & & $\checkmark$ & \multirow[t]{2}{*}{$\checkmark$} & $\checkmark$ \\
\hline & Thermal Desorption & & $\checkmark$ & & & $\checkmark$ & & $\checkmark$ \\
\hline & Retorting for Mercury & & $\checkmark$ & & & $\checkmark$ & $\checkmark$ & $\checkmark$ \\
\hline & Materials Handling/Soil Washing/Gravity & & $\checkmark$ & & & $\checkmark$ & $\checkmark$ & $\bar{\checkmark}$ \\
\hline & Separation & & & & & & & \\
\hline & & & & te Accep & & & Residua & \\
\hline Institute for Envir & ntal Health and Safety & $\begin{array}{l}\text { Maturity } \\
\text { Level }\end{array}$ & $\begin{array}{l}\text { Low } \\
\text { Level } \\
\text { Waste } \\
\end{array}$ & $\begin{array}{l}\text { Trans- } \\
\text { uranic } \\
\text { Waste }\end{array}$ & $\begin{array}{c}\text { High } \\
\text { Level } \\
\text { Waste }\end{array}$ & $\begin{array}{c}\text { Ready } \\
\text { to } \\
\text { Dispose } \\
\end{array}$ & $\begin{array}{l}\text { Need } \\
\text { Treat- } \\
\text { ment }\end{array}$ & $\begin{array}{c}\text { Retumed } \\
\text { to } \\
\text { Gen. } \\
\end{array}$ \\
\hline Chemical Oxidation & Chemical Photolysis & Bench & & & & & & $\checkmark$ \\
\hline Chemical Oxidation & Fenton's Reagent Oxidation & Bench & & & & & & $\checkmark$ \\
\hline
\end{tabular}


Table 2-2 (Continued)

Treatability Study Commercial Providers Capabilities

\section{IT Corporation}

Amalgamation

Biodegradation

Biodegradation

Chemical Oxidation

Deactivation

Debris Washing

Incineration

Macroencapsulation

Organic Destruction

Organic Destruction

Organic Destruction

Recovery of Metals

Recovery of Metals

Recovery of Metals

Recovery of Organics

Recovery of Organics

Retorting for Mercury

Soil Washing

Stabilization

Steam Stripping

Vitrification

Wet Oxidation

M4 Environmental Management Inc.

Recovery of Metals
Amalgamation

Excavated Soil Biotreatment (BIOFAST)

Natural Attenuation

UV Photolysis

Deactivation

Debris Decontamination

Incineration

Macroencapsulation

Base Catalyzed Decomposition (BCD)

Chemical Dehalogenation

UV Photolysis/Chemical Oxidation

Electro-Chemical Separation

Precipitation

Reverse Osmosis

Adsorption Media

Chemical Extraction, with or without chelants

Low Temperature Thermal Desorption

Soil Washing/Flushing

Stabilization/ Solidification

Steam/Air Stripping

Vitrification

Wet Air Oxidation

\begin{tabular}{|c|c|c|c|}
\hline \multirow[b]{2}{*}{$\begin{array}{l}\text { Maturity } \\
\text { Level }\end{array}$} & \multicolumn{3}{|c|}{ Waste Accepted } \\
\hline & $\begin{array}{l}\text { Low } \\
\text { Level } \\
\text { Wastc }\end{array}$ & $\begin{array}{l}\text { Trans- } \\
\text { uranic } \\
\text { Waste }\end{array}$ & $\begin{array}{l}\text { High } \\
\text { Level } \\
\text { Waste }\end{array}$ \\
\hline Commercial & $\checkmark$ & $\checkmark$ & \\
\hline Commercial & $\checkmark$ & $\checkmark$ & \\
\hline Commercial & $\checkmark$ & $\checkmark$ & \\
\hline Commercial & $\checkmark$ & $\checkmark$ & \\
\hline Commercial & $\checkmark$ & $\checkmark$ & \\
\hline Commercial & $\checkmark$ & $\checkmark$ & \\
\hline Commercial & $\checkmark$ & $\checkmark$ & \\
\hline Commercial & $\checkmark$ & $\checkmark$ & \\
\hline Commercial & $\checkmark$ & $\checkmark$ & \\
\hline Commercial & $\checkmark$ & $\checkmark$ & \\
\hline Commercial & $\checkmark$ & $\checkmark$ & \\
\hline Bench & $\checkmark$ & $\checkmark$ & \\
\hline Commercial & $\checkmark$ & $\checkmark$ & \\
\hline Commercial & $\checkmark$ & $\checkmark$ & \\
\hline Commercial & $\checkmark$ & $\checkmark$ & \\
\hline Commercial & $\checkmark$ & $\checkmark$ & \\
\hline
\end{tabular}

Commercial

Commercial

Commercial

Commercial

Bench

Commercial

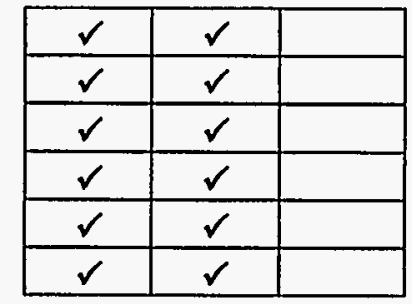

Waste Accepted

Maturity

Level

Quantum - Catalytic Extraction Processing

Commercial
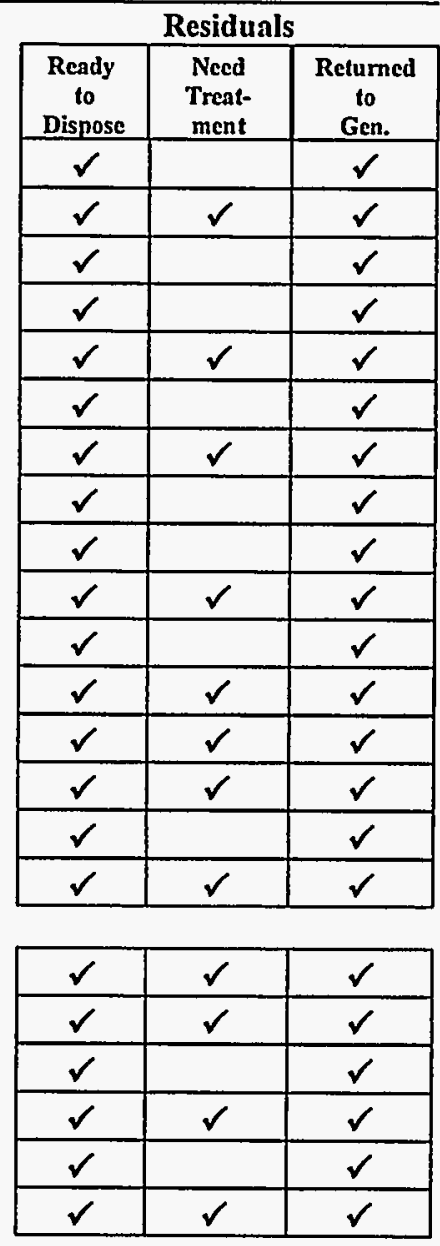

Residuals

\begin{tabular}{|c|c|c|}
\hline $\begin{array}{c}\text { Ready } \\
\text { to } \\
\text { Dispose }\end{array}$ & $\begin{array}{c}\text { Need } \\
\text { Treat- } \\
\text { ment }\end{array}$ & $\begin{array}{c}\text { Returned } \\
\text { to } \\
\text { Gen. }\end{array}$ \\
\hline$\checkmark$ & & \\
\hline
\end{tabular}


Table 2-2 (Continued)

Treatability Study Commercial Providers Capabilities

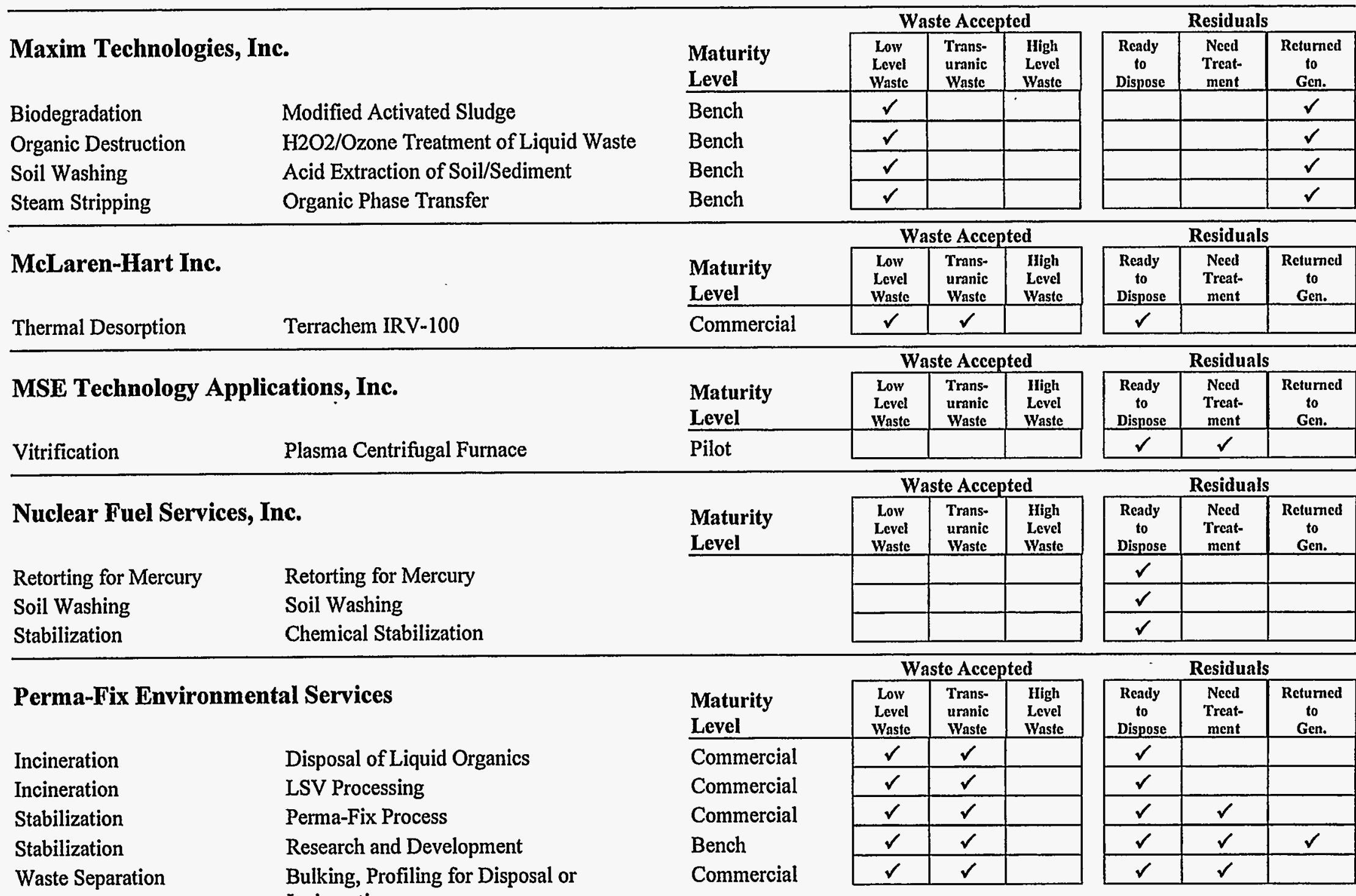


Table 2-2 (Continued)

Treatability Study Commercial Providers Capabilities

\begin{tabular}{|c|c|c|c|c|c|c|c|c|}
\hline \multirow{2}{*}{\multicolumn{2}{|c|}{ Radian Corporation }} & \multirow[b]{2}{*}{$\begin{array}{l}\text { Maturity } \\
\text { Level }\end{array}$} & \multicolumn{3}{|c|}{ Waste Accepted } & \multicolumn{3}{|c|}{ Residuals } \\
\hline & & & $\begin{array}{l}\text { Low } \\
\text { Level } \\
\text { Waste } \\
\end{array}$ & $\begin{array}{l}\text { Trans- } \\
\text { uranic } \\
\text { Waste. } \\
\end{array}$ & $\begin{array}{l}\text { High } \\
\text { Level } \\
\text { Waste } \\
\end{array}$ & $\begin{array}{c}\text { Ready } \\
\text { to } \\
\text { Dispose } \\
\end{array}$ & $\begin{array}{l}\text { Need } \\
\text { Treat- } \\
\text { ment }\end{array}$ & $\begin{array}{c}\text { Refurned } \\
\text { to } \\
- \text { Gen. } \\
\end{array}$ \\
\hline Biodegradation & Research and Development & Bench & $\checkmark$ & $\checkmark$ & & & & $\checkmark$ \\
\hline Chemical Oxidation & Research and Development & Bench & $\checkmark$ & $\checkmark$ & & & & $\checkmark$ \\
\hline Stabilization & Research and Development & Bench & $\checkmark$ & $\checkmark$ & & & & $\checkmark$ \\
\hline \multirow{2}{*}{\multicolumn{2}{|c|}{ Science Applications International Corporation }} & & \multicolumn{3}{|c|}{ Waste Accepted } & \multicolumn{3}{|c|}{ Residuals } \\
\hline & & $\begin{array}{l}\text { Maturity } \\
\text { Level }\end{array}$ & $\begin{array}{l}\text { Low } \\
\text { Level } \\
\text { Waste } \\
\end{array}$ & $\begin{array}{l}\text { Trans- } \\
\text { uranic } \\
\text { Waste }\end{array}$ & $\begin{array}{l}\text { Iligh } \\
\text { Lcrel } \\
\text { Waste } \\
\end{array}$ & $\begin{array}{c}\text { Ready } \\
\text { to } \\
\text { Dispose }\end{array}$ & $\begin{array}{c}\text { Need } \\
\text { Treat- } \\
\text { ment }\end{array}$ & $\begin{array}{c}\text { Retumed } \\
\text { to } \\
\text { Gen. }\end{array}$ \\
\hline Vitrification & Plasma Hearth Process & Pilot & & & & $\checkmark$ & & \\
\hline \multirow{2}{*}{\multicolumn{2}{|c|}{ Scientific Ecology Group, Inc. }} & & \multicolumn{3}{|c|}{ Waste Accepted } & \multicolumn{3}{|c|}{ Residuals } \\
\hline & & $\begin{array}{l}\text { Maturity } \\
\text { Level } \\
\end{array}$ & $\begin{array}{l}\text { Low } \\
\text { Level } \\
\text { Waste } \\
\end{array}$ & 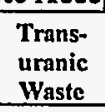 & $\begin{array}{l}\text { High } \\
\text { Level } \\
\text { Waste } \\
\end{array}$ & $\begin{array}{c}\text { Ready } \\
\text { to } \\
\text { Dispose }\end{array}$ & $\begin{array}{c}\text { Need } \\
\text { Treat- } \\
\text { ment }\end{array}$ & $\begin{array}{c}\text { Returned } \\
\text { to } \\
\text { Gen. }\end{array}$ \\
\hline \multirow{9}{*}{$\begin{array}{l}\text { Organic Destr } \\
\text { Stabilization } \\
\text { Stabilization } \\
\text { Stabilization } \\
\text { Stabilization } \\
\text { Stabilization } \\
\text { Stabilization } \\
\text { Vitrification } \\
\text { Vitrification }\end{array}$} & Steam Reforming & \multirow{9}{*}{$\begin{array}{l}\text { Commercial } \\
\text { Commercial } \\
\text { Commercial } \\
\text { Pilot } \\
\text { Commercial } \\
\text { Pilot } \\
\text { Pilot } \\
\text { Commercial } \\
\text { Commercial }\end{array}$} & $\checkmark$ & $\checkmark$ & $\checkmark$ & $\checkmark$ & & \\
\hline & Cement Stabilization & & $\checkmark$ & $\checkmark$ & & $\checkmark$ & $\dot{-}$ & \\
\hline & Magnesium Oxide Cement Stabilization & & $\checkmark$ & $\checkmark$ & & $\checkmark$ & & \\
\hline & Polyethylene Stabilization & & $\checkmark$ & & & $\checkmark$ & & \\
\hline & Thermoplastic Epoxy Stabilization & & $\checkmark$ & & & & $\checkmark$ & \\
\hline & Thermoset Epoxy Stabilization & & $\checkmark$ & & & & $\checkmark$ & \\
\hline & Vinyl Ester Stabilization & & $\checkmark$ & & & $\checkmark$ & & \\
\hline & Joule-Heated Melter & & $\checkmark$ & $\checkmark$ & $\checkmark$ & & $\checkmark$ & \\
\hline & Plasma-Driven Reactor & & $\checkmark$ & $\checkmark$ & $\checkmark$ & $\checkmark$ & & \\
\hline \multirow[b]{2}{*}{ Selentec } & & & \multicolumn{3}{|c|}{ Waste Accepted } & \multicolumn{3}{|c|}{ Residuals } \\
\hline & & $\begin{array}{l}\text { Maturity } \\
\text { Level } \\
\end{array}$ & $\begin{array}{l}\text { Low } \\
\text { Level } \\
\text { Waste } \\
\end{array}$ & $\begin{array}{l}\text { Truns- } \\
\text { uranic } \\
\text { Waste } \\
\end{array}$ & $\begin{array}{l}\text { High } \\
\text { Level } \\
\text { Waste } \\
\end{array}$ & $\begin{array}{c}\text { Ready } \\
10 \\
\text { Dispose } \\
\end{array}$ & $\begin{array}{l}\text { Necd } \\
\text { Treat- } \\
\text { ment }\end{array}$ & $\begin{array}{c}\text { Returned } \\
\text { to } \\
\text { Gen. }\end{array}$ \\
\hline Extraction & Electrochemical Ion Exchange (EIX) & Pilot & $\checkmark$ & $\checkmark$ & & & & \\
\hline Recovery of Metals & MAG*SEP & Pilot & $\checkmark$ & $\checkmark$ & & $\checkmark$ & & \\
\hline Soil Washing & $\mathrm{ACT} * \mathrm{DE} * \mathrm{CON}$ & Pilot & $\checkmark$ & $\checkmark$ & & & $\checkmark$ & \\
\hline
\end{tabular}


Table 2-2 (Continued)

Treatability Study Commercial Providers Capabilities

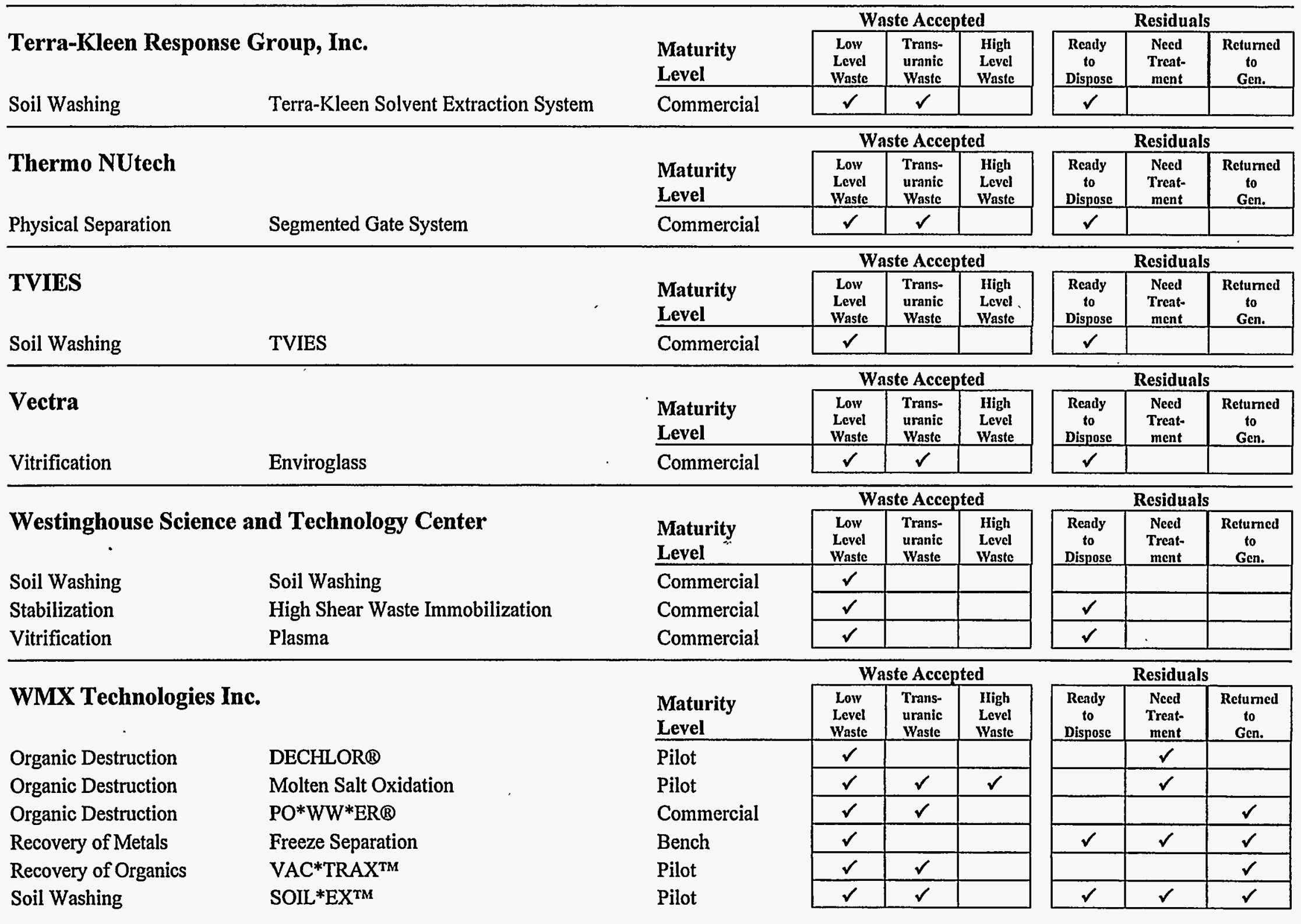


Table 2-2 (Continued)

Treatability Study Commercial Providers Capabilities

Stabilization

Stabilization

Vitrification

Vitrification

\section{CHEM MATRIX}

Phoenix Ash Technology (TIDE Bricks)

Cold Crucible Vitrification

Joule-Heated Melters
Commercial

Bench

Pilot

Commercial

\begin{tabular}{|c|c|c|}
\hline$\checkmark$ & $\checkmark$ & \\
\hline$\checkmark$ & $\checkmark$ & \\
\hline$\checkmark$ & $\checkmark$ & $\checkmark$ \\
\hline$\checkmark$ & $\checkmark$ & $\checkmark$ \\
\hline
\end{tabular}

\begin{tabular}{|l|l|l|}
\hline$\checkmark$ & & \\
\hline$\checkmark$ & & \\
\hline$\checkmark$ & & \\
\hline$\checkmark$ & & \\
\hline
\end{tabular}


Table 2-3

Treatability Study Commercial Provider Waste Matrix Capabilities

Alternative Remedial

Technologies

Soil Washing

\section{Atomic Energy of Canada \\ Limited (AECL)}

CHEMICTM

\section{Babcock \& Wilcox, A McDermott Company}

Cyclone Furnace Vitrification

Soil Screening/Washing

Brown and Root Environmental Air Sparging/Soil Vapor Extraction

All Technologies

Auger Mixing System

Fracturing to Increase Premeability in $\mathrm{Be}$ In-situ Chemical Treatment Jet Grouting

Permeable treatment bed installation

Pressure Grouting of Soil and Fractured

Soil Rake

Soil Saw Barrier System

Carlo Environmental

Technologies, Inc.

Superset

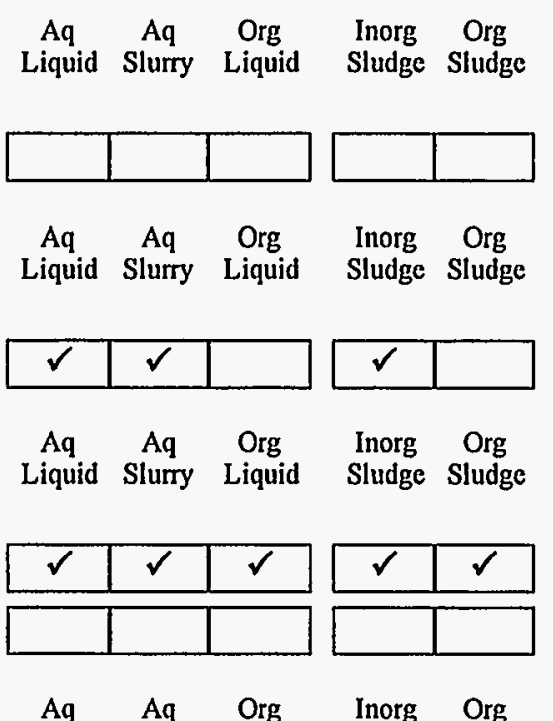

Soil Metal Other

Lab React

Packs Metal

$\mathrm{Hg}$

$\mathrm{Pb} \quad \mathrm{Cd}$

Bat Bat

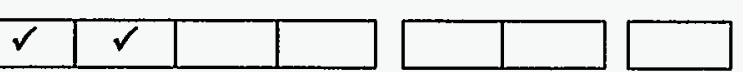

Soil Metal Other Lab React Packs Metal

$\mathrm{Hg}$

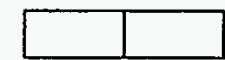

$\mathrm{Pb} \quad \mathrm{Cd}$

Bat Bat

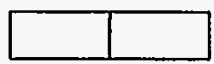

$\mathrm{Pb} \quad \mathrm{Cd}$

Bat Bat

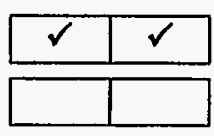

$\begin{array}{cc}\mathrm{Pb} & \mathrm{Cd} \\ \mathrm{Bat} & \text { Bat }\end{array}$
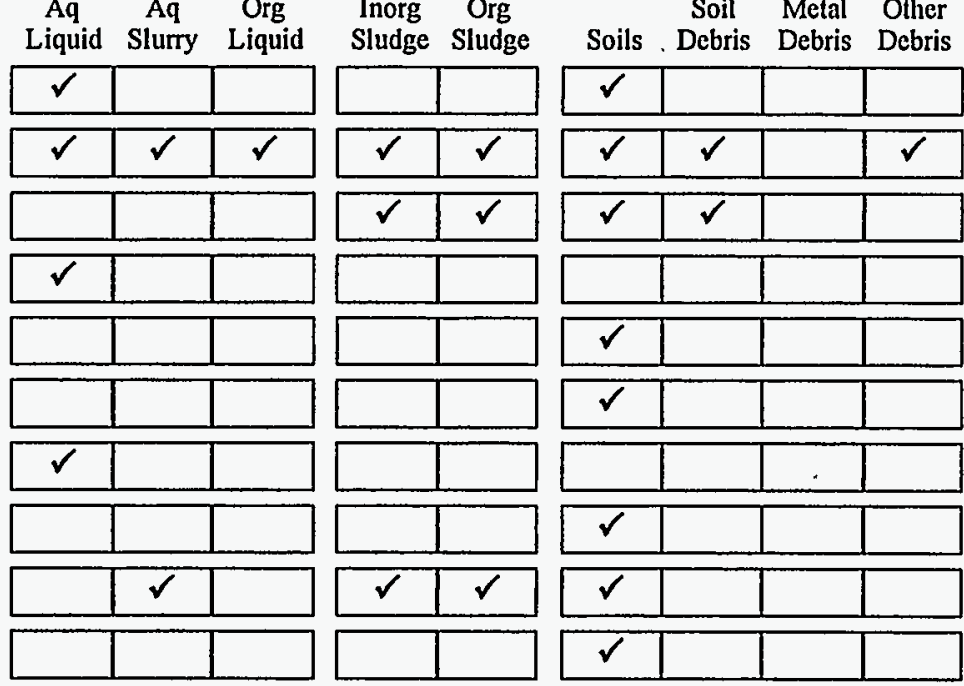

Lab React

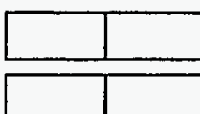

$\square$
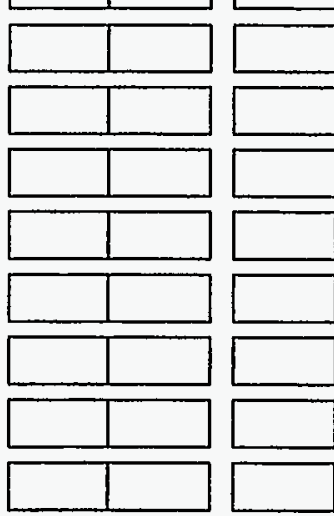

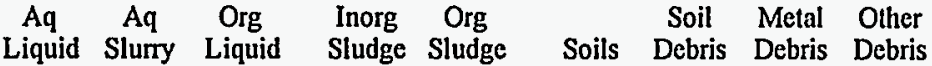

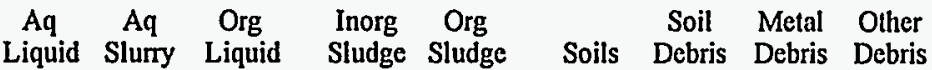

Lab React

Packs Metal

$\mathrm{Hg}$

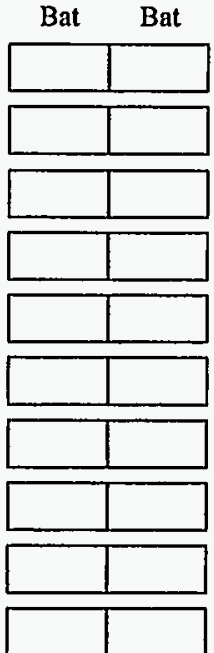

$\mathrm{Pb} \quad \mathrm{Cd}$
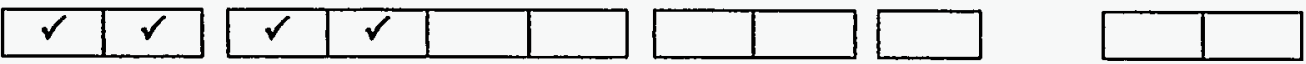
Table 2-3 (Continued)

Treatability Study Commercial Provider Waste Matrix Capabilities

\section{CF Systems}

Liquified Gas Solvent Extraction

Chemical Waste Management, Inc.

Industrial boiler used for energy recovery

Earth Resources Corporation

Compressed Gases

Eichrom Industries, Inc.

Diphonix Ion Exchange

Electro-Pyrolysis, Inc.

DC Graphite Arc Melter

\section{Energy and Environmental} Research Center

Activated Carbon

Centrifugal Membrane Filtration

Fluid-Bed Calcination

Hydrothermal Boiler

Pyrolysis

\section{Envirocare of Utah, Inc.}

Macroencapsulation

Non-Thermal Stabilization

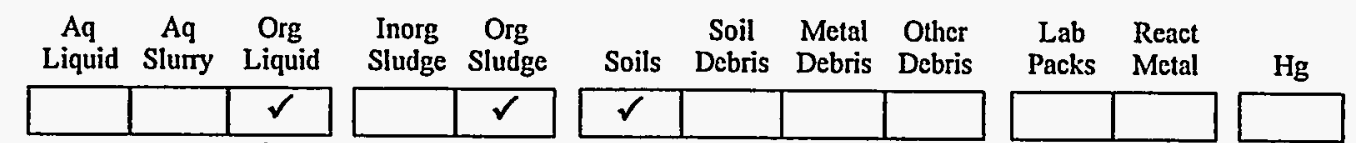

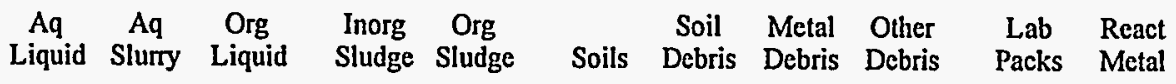

$\mathrm{Hg}$

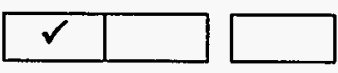

$\begin{array}{cccc}\text { Aq } & \text { Aq } & \text { Org } & \text { Inorg } \\ \text { Liquid } & \text { Org } \\ \text { Slurry } & \text { Liquid } & \text { Sludge } & \text { Sludge }\end{array}$
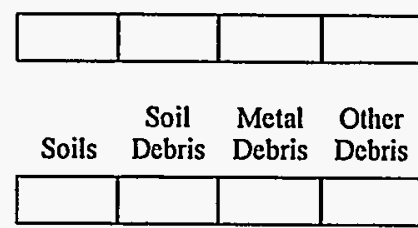

$\begin{array}{cc}\text { Lab } & \text { React } \\ \text { Packs } & \text { Metal }\end{array}$

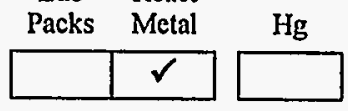

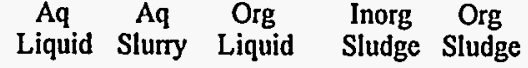

Soil Metal Other

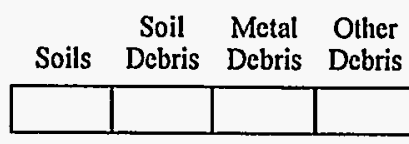

Lab React

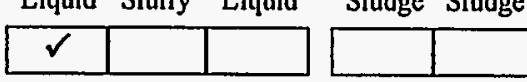

Aq Aq Org Inorg Org

\begin{tabular}{|l|l|l|}
\hline Liquid Slurry & \multicolumn{1}{l}{ Liquid } \\
\hline & & \\
\hline
\end{tabular}

\begin{tabular}{|c|c|}
\multicolumn{2}{|c}{ Sludge Sludge } \\
\hline$\checkmark$ & $\checkmark$ \\
\hline
\end{tabular}

Soil Metal Other

\begin{tabular}{|c|c|c|c|} 
Soils & Debris & Debris Debris \\
\hline$\checkmark$ & $\checkmark$ & $\checkmark$ & $\checkmark$ \\
\hline
\end{tabular}

Aq Aq Org

Inorg Org

$\begin{array}{cccc}\text { Soil } & \text { Metal } & \text { Other } \\ \text { Soils } & \text { Debris } & \text { Debris } & \text { Debris }\end{array}$ Liquid Slurry Liquid Sludge Sludge

Packs Mctal

$\mathrm{Hg}$
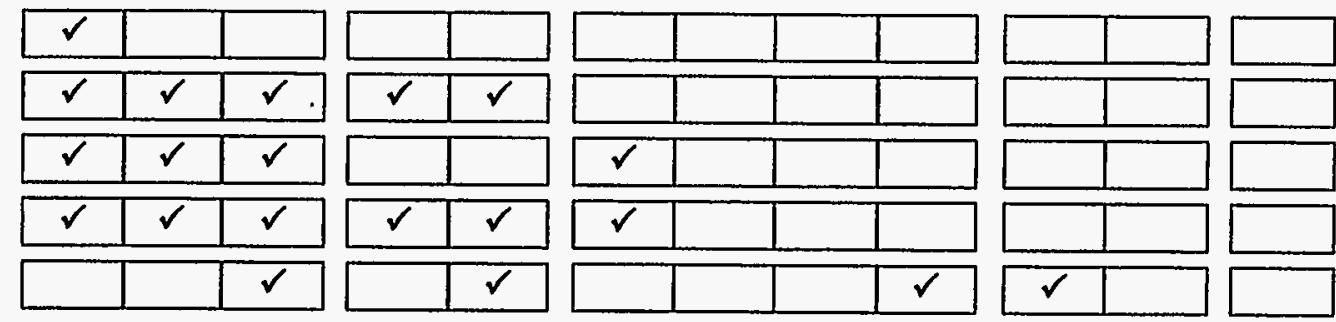

Aq Aq Org Inorg Org
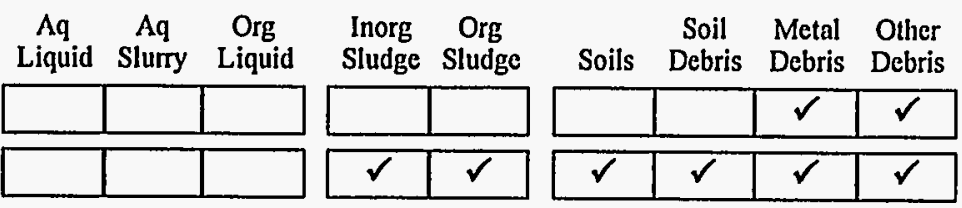

Lab React

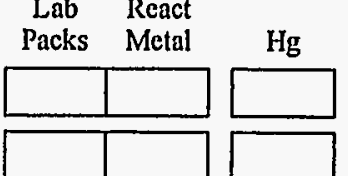

$\mathrm{Pb} \quad \mathrm{Cd}$

Bat Bat

$\mathrm{Pb} \quad \mathrm{Cd}$

Bai Bat
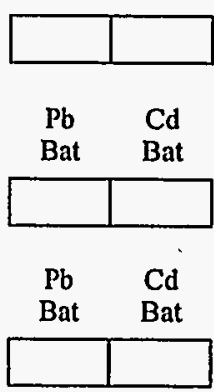

$\mathrm{Pb} \quad \mathrm{Cd}$

Bat Bat

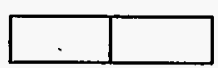

$\mathrm{Pb} \quad \mathrm{Cd}$

Bat Bat
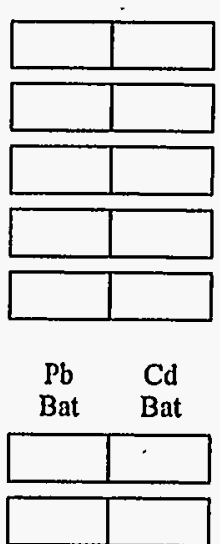
Table 2-3 (Continued)

Treatability Study Commercial Provider Waste Matrix Capabilities

Fluid Tech, Inc.

Aquaset/Petroset

Geosafe Corporation

In Situ Vitrification (ISV)

Hazen Research Inc.

Chemical Leaching

Incineration/Combustion/Calcining/Roast

Materials Handling/Soil Washing/Gravity

Retorting for Mercury

Solvent Extraction

Thermal Desorption

Institute for Environmental

Health and Safety

Chemical Photolysis

Fenton's Reagent Oxidation

\section{IT Corporation}

Adsorption Media

Amalgamation

Base Catalyzed Decomposition (BCD)

Chemical Dehalogenation

Chemical Extraction, with or without che

Deactivation

Debris Decontamination

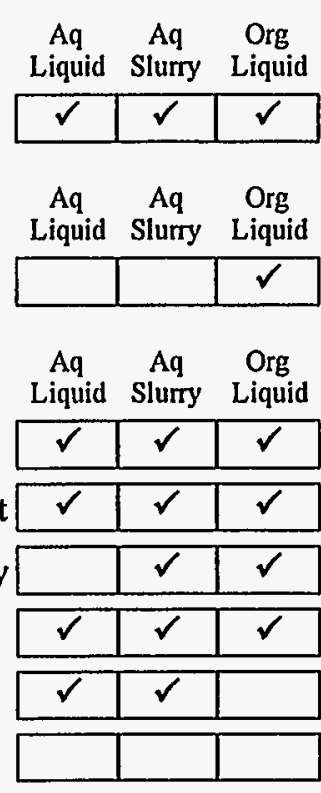

Aq Aq Org

Liquid Slurry Liquid
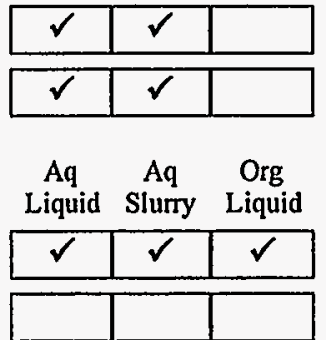

\begin{tabular}{|l|l|l|}
\hline$\checkmark$ & $\checkmark$ & $\checkmark$ \\
\hline & & $\checkmark$ \\
\hline
\end{tabular}

\begin{tabular}{|c|c|c|}
\hline$\checkmark$ & $\checkmark$ & $\checkmark$ \\
\hline$\checkmark$ & $\checkmark$ & $\checkmark$ \\
\hline
\end{tabular}

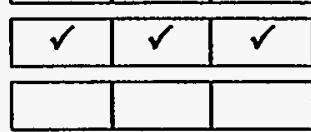

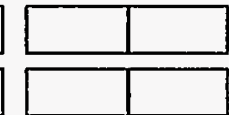

$\begin{array}{cc}\text { Inorg } & \text { Org } \\ \text { Sludge } & \text { Sludge }\end{array}$

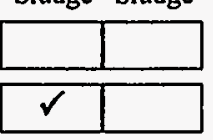

\begin{tabular}{|l|l|}
\hline & $\checkmark$ \\
\hline & \\
\hline
\end{tabular}

Inorg Org \begin{tabular}{|l|l|}
\hline$r$ & $r$ \\
\hline
\end{tabular}

Inorg Org

\begin{tabular}{|l|l|}
\hline$\checkmark$ & $\checkmark$ \\
\hline
\end{tabular}

Inorg Org

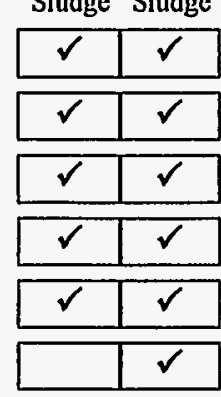

Inorg Org Sludge Sludge

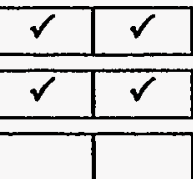

Soil Metal Other Lab React

\begin{tabular}{|c|c|c|c|c|c|c|}
\hline Soils & Debris & Debris & Debris & Packs & Metal & $\mathrm{Hg}$ \\
\hline $\bar{\checkmark}$ & $\bar{\checkmark}$ & $\checkmark$ & $\bar{\checkmark}$ & $\bar{\checkmark}$ & & $\checkmark$ \\
\hline
\end{tabular}

Soil Metal Other

Soils
\begin{tabular}{|c|c|c|c|}
\hline$\checkmark$ & $\checkmark$ & $\checkmark$ & $\checkmark$ \\
\hline
\end{tabular}

Lab React
Packs

Soil Metal Other

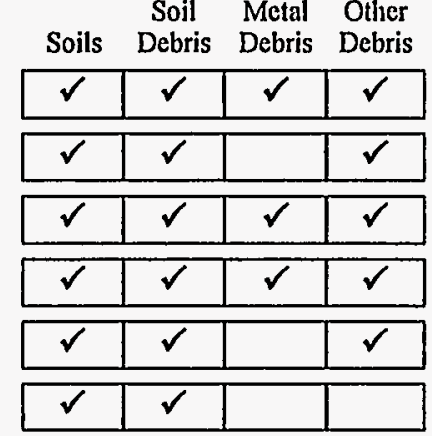

Soil Metal Other Soils Debris Debris Debris
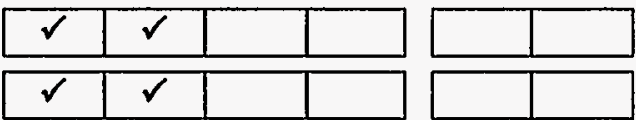

Soil Metal Other

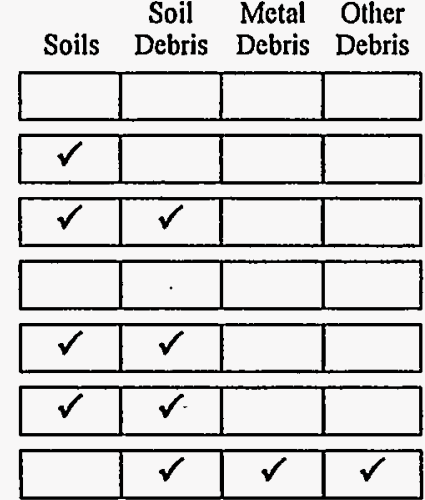

\begin{tabular}{|c|c|c|}
\hline$\checkmark$ & $\checkmark$ \\
\hline
\end{tabular}

$\begin{array}{ccc}\text { Lab } & \text { React } \\ \text { Packs } & \text { Metal } \quad \mathrm{Hg}\end{array}$
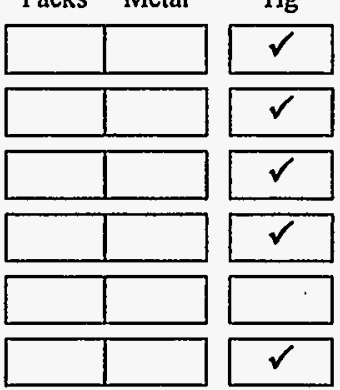

Lab React

Packs Metal

$\mathrm{Pb} \quad \mathrm{Cd}$
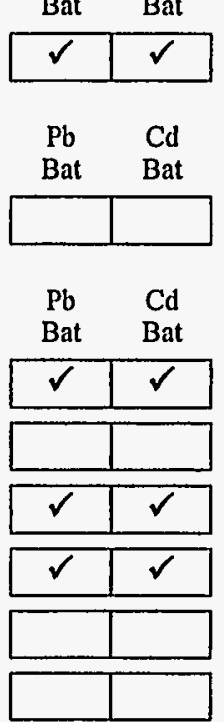

$\mathrm{Pb} \quad \mathrm{Cd}$

Bat Bat

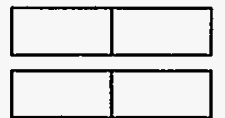

Lab React Packs Metal
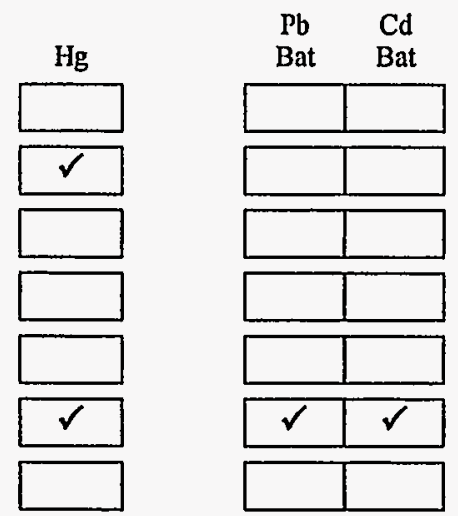
Table 2-3 (Continued)

Treatability Study Commercial Provider Waste Matrix Capabilities

Electro-Chemical Separation

Excavated Soil Biotreatment (BIOFAST)

Incineration

Low Temperature Thermal Desorption

Macroencapsulation

Natural Attenuation

Precipitation

Reverse Osmosis

Soil Washing/Flushing

Stabilization/ Solidification

Steam/Air Stripping

UV Photolysis

UV Photolysis/Chemical Oxidation

Vitrification

Wet Air Oxidation

\section{M4 Environmental}

Management Inc.

Quantum - Catalytic Extraction Processin

Maxim Technologies, Inc.

Acid Extraction of Soil/Sediment

H2O2/Ozone Treatment of Liquid Waste

Modified Activated Sludge

Organic Phase Transfer

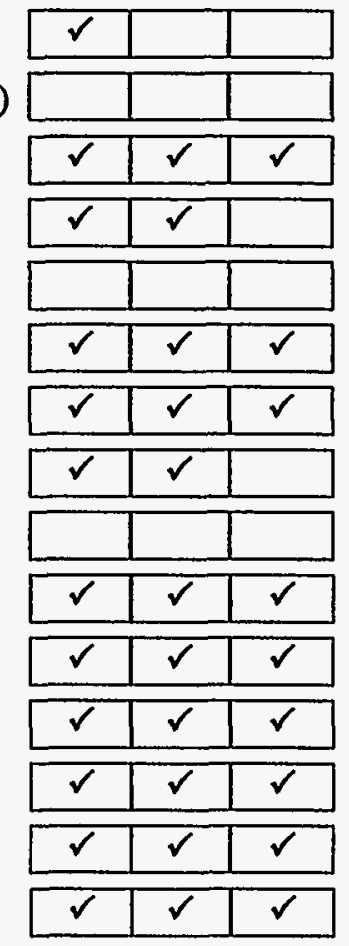

Aq Aq Org Inorg Org $\begin{array}{ccc}\text { Aq } & \text { Aq } & \text { Org } \\ \text { Liquid Slumy }\end{array}$

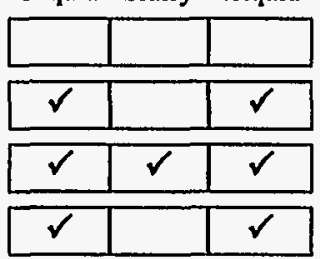

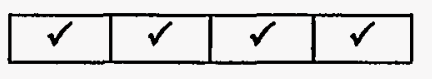

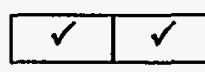

Inorg Org Soil Mctal Other Soils Debris Debris Debris Packs Metal
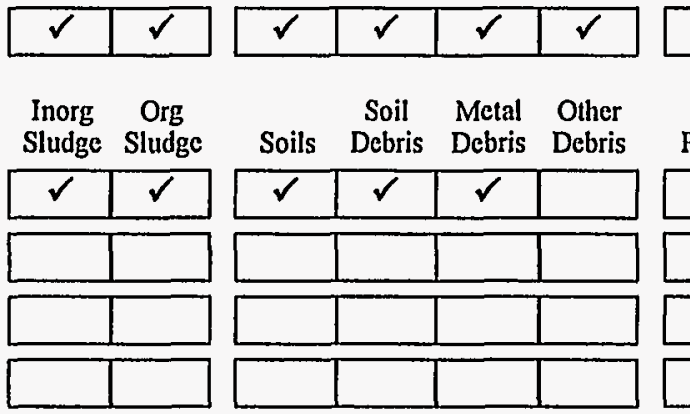

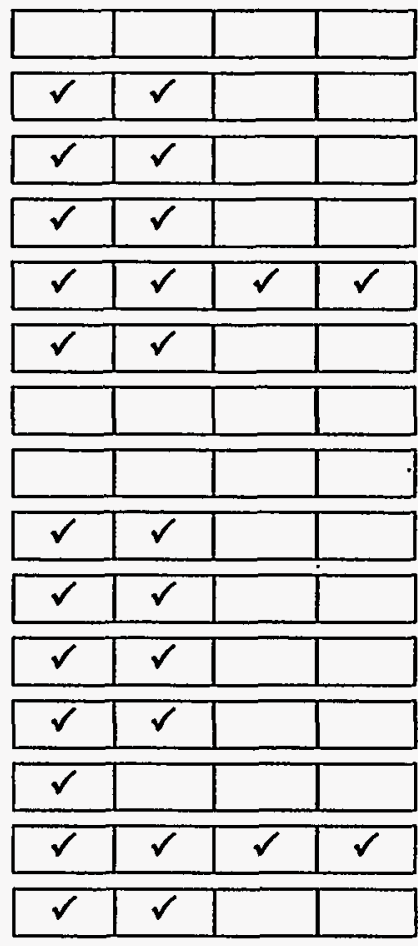

Soil Metal Other Soils Debris Debris Debris

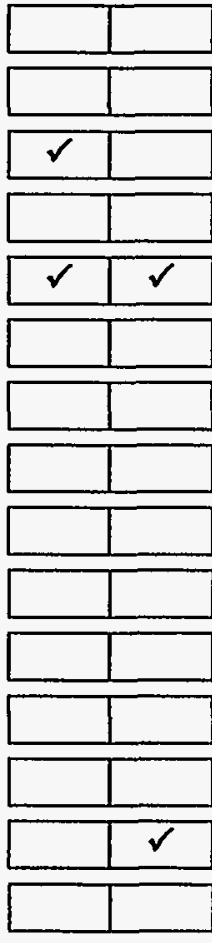

Lab React Packs Metal

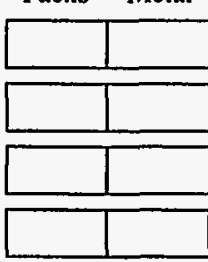

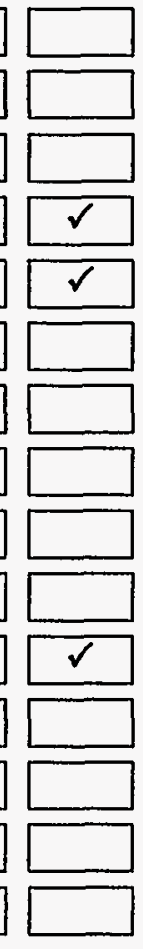

$\mathrm{Hg}$

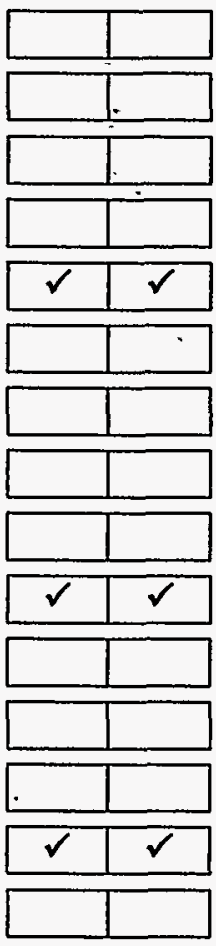

$\mathrm{Pb} \quad \mathrm{Cd}$

Bat Bat

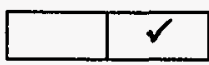

$\mathrm{Pb} \quad \mathrm{Cd}$

Bat Bat

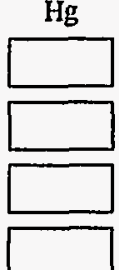


Table 2-3 (Continued)

Treatability Study Commercial Provider Waste Matrix Capabilities

McLaren-Hart Inc.

Terrachem IRV-100

MSE Technology Applications, Inc.

Plasma Centrifugal Furnace

Nuclear Fuel Services, Inc.

Chemical Stabilization

Retorting for Mercury

Soil Washing

\section{Perma-Fix Environmental} Services

Bulking, Profiling for Disposal or Inciner

Disposal of Liquid Organics

LSV Processing

Perma-Fix Process

Research and Development

\section{Radian Corporation}

Research and Development

Research and Development

Research and Development

\section{Science Applications} International Corporation

Plasma Hearth Process
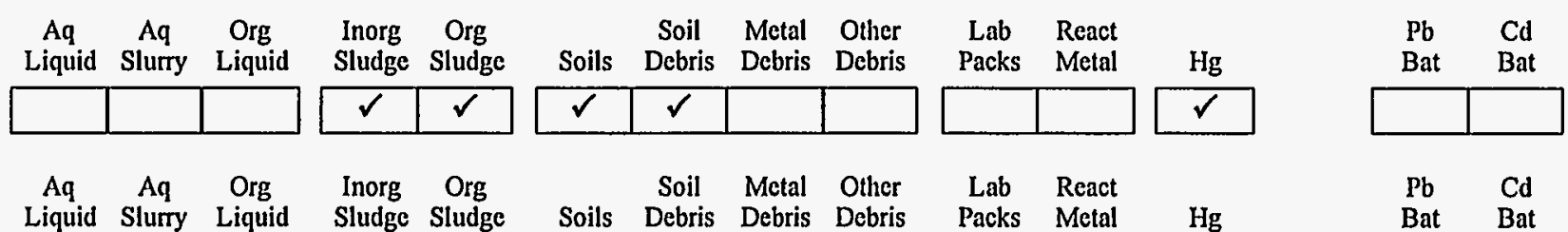

$\mathrm{Pb} \quad \mathrm{Cd}$

Bat Bat
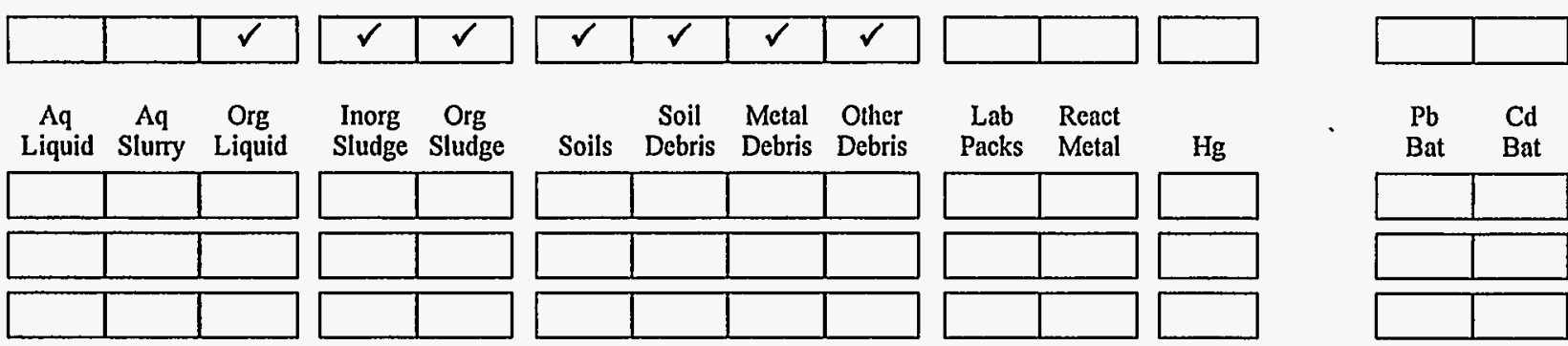

Inorg Org

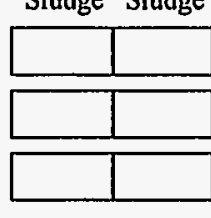

$\mathrm{Aq} \quad \mathrm{Aq}$ Org

Inorg Org

Soil Metal Other

Lab React

Liquid Slurry Liquid Sludge Sludge

Soils Debris Debris Debris


Aq Aq Org Inorg Org

Soil Metal Other

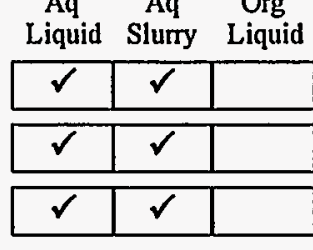

Sludge Sludge

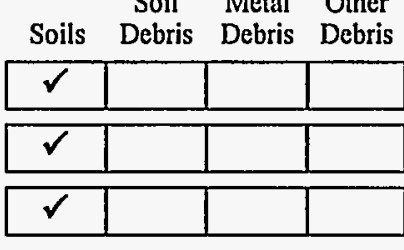

Lab React
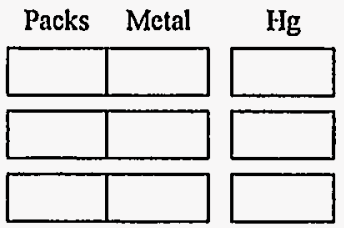

$\begin{array}{ccc}\text { Aq } & \text { Aq } & \text { Org } \\ \text { Liquid } & \text { Slurry } & \text { Liquid }\end{array}$

Soil Metal Other

Lab React

Soils Debris Debris Debris

Packs Metal

$\mathrm{Hg}$

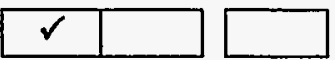

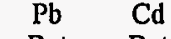


Bat Bat
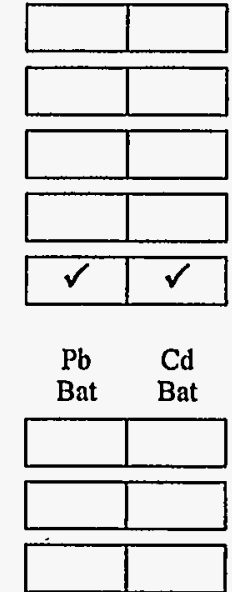

$\mathrm{Pb} \quad \mathrm{Cd}$

Bat Bat

\begin{tabular}{|l|l|l|}
\hline & $\checkmark$ & $\checkmark$ \\
\hline$\checkmark$ & $\checkmark$ \\
\hline
\end{tabular} \begin{tabular}{|l|l|l|l|l|}
\hline$\checkmark$ & $\checkmark$ & $\checkmark$ & $\checkmark$ \\
\hline
\end{tabular}


Table 2-3 (Continued)

Treatability Study Commercial Provider Waste Matrix Capabilities

Scientific Ecology Group, Inc.

Cement Stabilization

Joule-Heated Melter

Magnesium Oxide Cement Stabilization

Plasma-Driven Reactor

Polyethylene Stabilization

Steam Reforming

Thermoplastic Epoxy Stabilization

Thermoset Epoxy Stabilization

Vinyl Ester Stabilization

\section{Selentec}

$\mathrm{ACT} * \mathrm{DE} * \mathrm{CON}$

Electrochemical Ion Exchange (EIX)

MAG*SEP

Terra-Kleen Response Group,

Inc.

Terra-Kleen Solvent Extraction System

Thermo NUtech

Segmented Gate System

TVIES

TVIES

Vectra

Enviroglass



Inorg Org
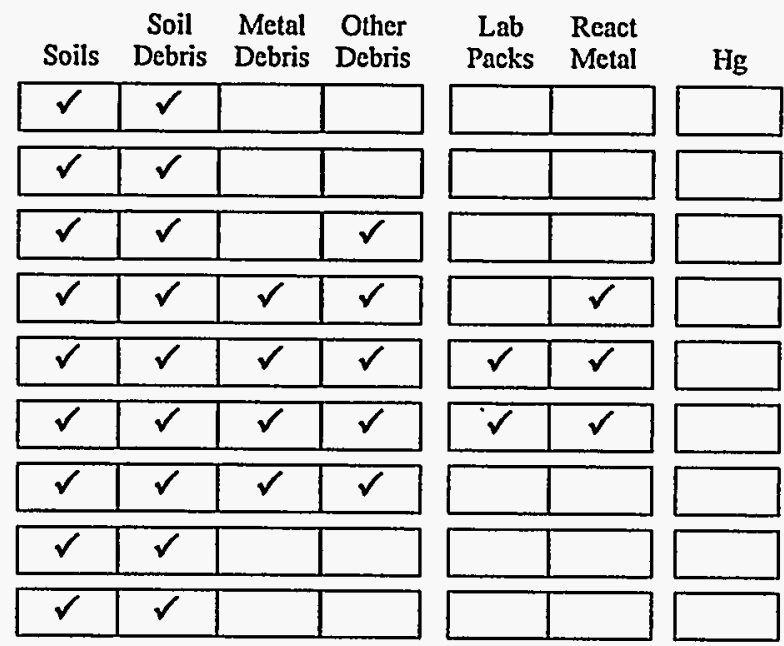

$\begin{array}{cccc}\text { Aq } & \text { Aq } & \text { Org } & \text { Inorg } \\ \text { Liquid } & \text { Org } \\ \text { Slurry } & \text { Liquid } & \text { Sludge } & \text { Sludge }\end{array}$

Liquid Slurry Liquid

Soil Metal Other

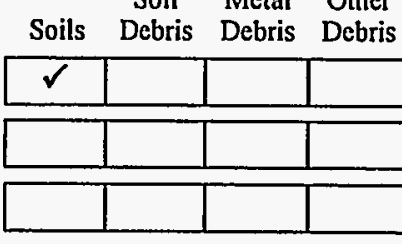

Soil Metal Other

Aq Aq Org

Liquid Slurry Liquid

Inorg Org

Sludge Sludge

Soils Debris Debris Debris
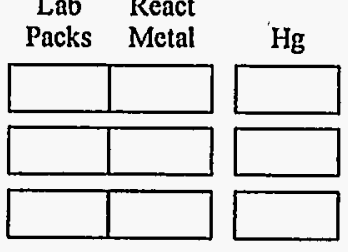

Lab React Packs Metal
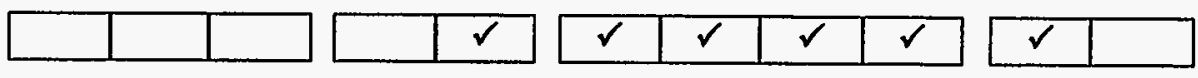



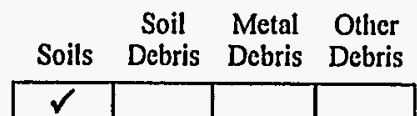

Lab React

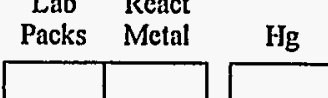



Soil Metal Other

Lab React

Packs Metal

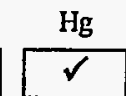

Aq Aq Org Inorg Org

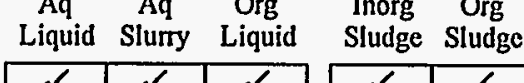

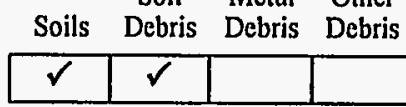

Soil Metal Other

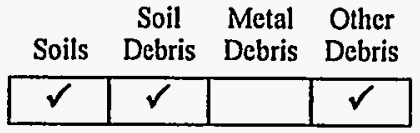

Lab React

Packs Metal

\begin{tabular}{|l|l|}
\hline$\checkmark$ & \\
\hline
\end{tabular}
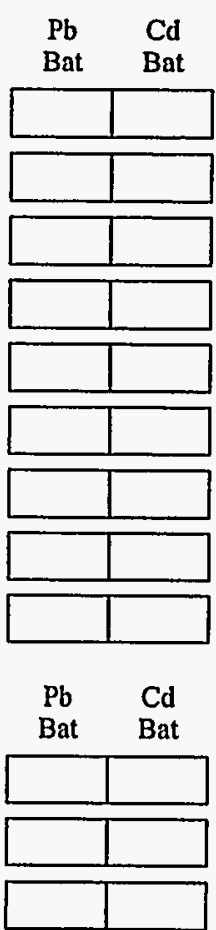

$\mathrm{Pb} \quad \mathrm{Cd}$

Bat Bat
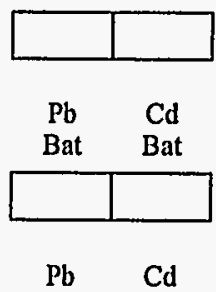

Bat Bat
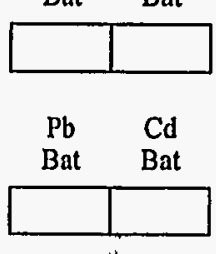
Table 2-3 (Continued)

Treatability Study Commercial Provider Waste Matrix Capabilities

\section{Westinghouse Science and Technology Center}

High Shear Waste Immobilization

Plasma

Soil Washing

WMX Technologies Inc. CHEM MATRIX

Cold Crucible Vitrification DECHLOR®

Freeze Separation

Joule-Heated Melters

Molten Salt Oxidation

Phoenix Ash Technology (TIDE Bricks) PO*WW*ER(B)

SOIL*EXTM

VAC*TRAXTM

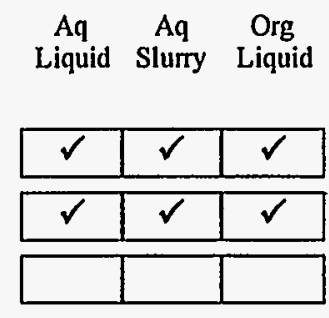

Inorg Org

$\begin{array}{cccc}\text { Soil } & \text { Metal } & \text { Other } \\ \text { Soils } & \text { Debris } & \text { Debris } & \text { Debris }\end{array}$

$\begin{array}{cc}\text { Lab } & \text { React } \\ \text { Packs } & \text { Metal }\end{array}$

$\mathrm{Hg}$

$\mathrm{Pb}$
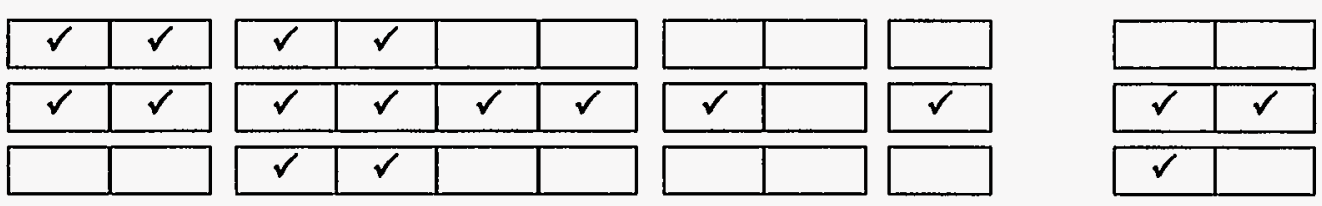

Aq Aq Org Inorg Org

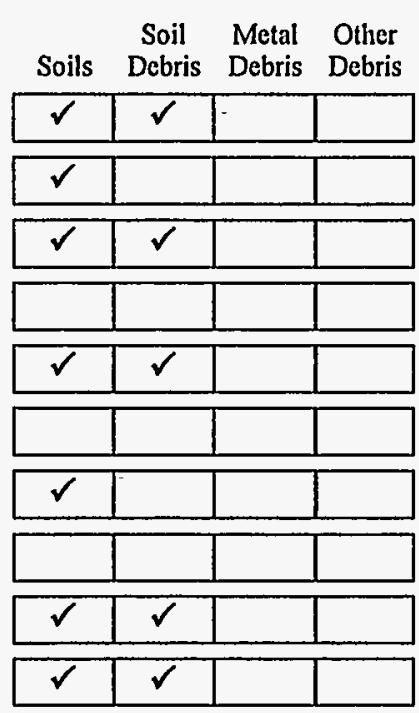

Lab React

\begin{tabular}{|l|l|}
\hline Packs & Metal \\
\hline &
\end{tabular}

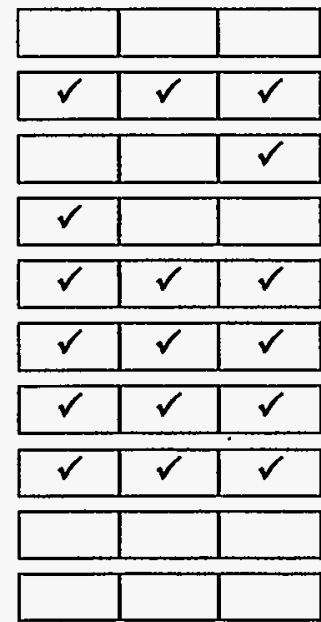

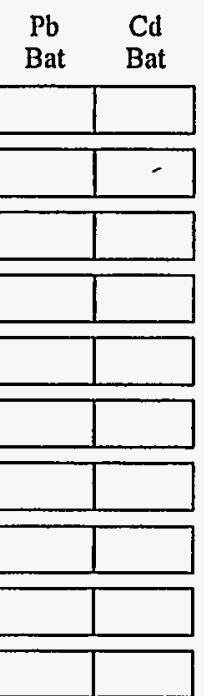


- Commercial mixed-waste treatment facilities that can perform treatability studies under their existing permits and licenses.

- Environmental restoration technology suppliers with experience at radioactive sites but that do not typically have their own mixed-waste treatability study facilities.

\subsection{Use of Summary Tables}

To use the results of this survey, the MWFA recommends a review of the data in Tables 2-1 and 2-2 to locate commercial providers with reported capability in the technologies of interest. Table 2-3 can then be used to evaluate whether the waste matrix can be treated by that provider/technology. Subsequently, review of the permit and license information reported in Attachment 2 is useful in determining whether the waste activity levels are compatible with the license limits. It should be noted that some commercial providers report that minor radioactive materials license modifications are quickly obtained, so the license limits given in Attachment 2 should be regarded as approximate. Lastly, telephone discussions with the listed contact can confirm the match of the waste problem with the technology/provider.

The following conclusions result from the data presented:

- One hundred six processes are offered by thirty-three firms for treatability studies in twenty-one of the EPA's general technology categories.

- Responses have included both waste management and environmental restoration technologies.

- Several commercial providers expect an NRC or state radioactive materials license very soon.

- Many commercial providers responded positively to the telephone screen but did not return the completed questionnaire.

- Radioactive license limits are typically radionuclide specific, but radioactive material possession as high as 10 Curies is available. The supplier must be consulted to ensure that all radionuclides are within license limits.

- Two commercial suppliers identified the ability to accept plutonium in gram quantities. 


\section{REGULATORY PROVISIONS}

DOE mixed-waste treatability studies, conducted in accordance with RCRA, are potentially subject to numerous other federal and state regulations, as well as DOE Orders. Treatability studies dealing with environmental restoration wastes generated at sites regulated under CERCLA will be subject to CERCLA requirements. If the treatability study involves polychlorinated biphenyls (PCB), requirements of TSCA will be applicable. If the study is conducted at a commercial treatability study laboratory or treatment facility, the facility must have a radioactive materials license from the $\mathrm{NRC}$ or applicable state agency. Waste samples transported to off-site facilities by common carriers must meet packaging requirements of the NRC and the DOT. The DOE is also responsible for complying with the provisions of the NEPA.

Regulatory issues related to treatability studies are depicted on a flow chart in Figure 3-1. If a treatability study is to be conducted off site, the generator should contact the off-site facility for specific regulatory protocols to be followed. The following discussion presents a more detailed review of regulations and requirements impacting treatability studies.

\subsection{Resource Conservation and Recovery Act}

\subsubsection{The Treatability Study Exemption}

RCRA provides for a treatability study exemption (in accordance with Title 40, Code of Federal Regulations, Part 261, Section 4, paragraph [e] \{40 CFR 261.4[e]\}) for generators. Generators that produce samples of RCRA-regulated hazardous waste for the purpose of having treatability studies performed on the samples are exempt from the requirements of 40 CFR 262, "Standards Applicable to Generators of Hazardous Waste," as well as from the requirements of $40 \mathrm{CFR} 261$ and 263 and RCRA notification requirements. Other requirements would apply, including transportation requirements, recordkeeping requirements, and biennial report preparation (for large-quantity generators or RCRA permitted facilities). Further, 40 CFR 261.4(d) provides exemptions for samples being tested to assess characteristics or composition.

In accordance with 40 CFR 261.4(e)(2)(I) and (ii), quantity limits do apply for treatability studies for each process being evaluated for each generated waste stream. These quantity limits may be exceeded if approved by the EPA Regional Administrator or the authorized state. It should be noted that the quantity limits stipulated by RCRA may or may not have been adopted and promulgated by the applicable states. State environmental regulations for both the DOE location where the waste originates and the location where the treatability study will be conducted may have more restrictive provisions than federal RCRA requirements (even including the absence of treatability study sample exemptions). Local regulations must be researched before shipment of the sample. 





RCRA regulations in 40 CFR 261.4(e) stipulate that the generator or treatability study provider use (for treatability studies) "no more than 10,000 kilograms $(\mathrm{kg})$ of media contaminated with nonacute hazardous waste, $1,000 \mathrm{~kg}$ of nonacute hazardous waste other than contaminated media, $1 \mathrm{~kg}$ of acute hazardous waste, or $2,500 \mathrm{~kg}$ of media contaminated with acute hazardous waste for each process being evaluated for each generated waste stream." (Acute hazardous waste is given an "H" designation in accordance with $40 \mathrm{CFR}$ Subpart D and is designated by EPA Hazardous Waste Numbers on the P-List of hazardous wastes as well as in F020, F021, F022, F023, F026, and F027.) The mass of each sample shipment must not exceed 10,000 kg. The 10,000-kg quantity may include media contaminated with nonacute hazardous waste, or may include a combination of the following - a total of $2,500 \mathrm{~kg}$ of media contaminated with acute hazardous waste, a total of $1,000 \mathrm{~kg}$ of hazardous waste (other than contaminated media), and a total of $1 \mathrm{~kg}$ of acute hazardous waste.

Further, in accordance with 40 CFR 261.4(f)(3), quantity limits per day on waste subjected to treatability studies must also be observed by the treatability study testing facility. Under this regulation, no more than a total of $10,000 \mathrm{~kg}$ of "as-received" media contaminated with nonacute hazardous waste, $2,500 \mathrm{~kg}$ of "asreceived" media contaminated with acute hazardous waste, or $250 \mathrm{~kg}$ of other "as-received" hazardous waste may be subject to initiation of treatment in all treatability studies in any single day. "As-received" waste is defined by RCRA as the waste as received in the shipment from the generator or sample collector. Per 40 CFR $261.4(f)(4)$, the total quantity of "as-received" waste stored at the testing facility may not exceed $10,000 \mathrm{~kg}$. Mobile treatment units (MTU) can qualify as testing facilities. Where a group of MTUs are located at the same site, the storage limit applies to all MTUs collectively (as though they were one unit). Exceedance of stipulated quantity limits by the generator or treatability study facility will subject either to RCRA treatment, storage, and disposal facility regulations governing management of hazardous waste.

The testing facility must either return the unused samples or residues to the sample originator within 90 days of completing the treatability study, or within 1 year of the date of shipment of the treatability study samples to the testing facility, or within 2 years for treatability studies involving bioremediation. Otherwise, the testing facility will become subject to regulations governing management of hazardous waste (if, in fact, the samples and residues are hazardous).

In accordance with $40 \mathrm{CFR}$ 261.4(f), samples undergoing treatability studies at laboratories and testing facilities are not subject to the requirements of 40 CFR 268 land disposal restrictions (LDR) as long as several criteria are met. These criteria include notification and reporting requirements, maintenance of records, maintenance of the treatability study contract, maintenance of shipping papers, receipt of an EPA Identification Number, and quantity limitations on how much waste can be treated per-unit-time, as well as stored per-unit-time. The criteria also specify that no hazardous waste may be placed on land or undergo open burning, and any unused samples or generated residues must either be managed as hazardous waste (if it is in fact hazardous) or returned to the sample originator, who must then manage the waste accordingly. 


\subsubsection{Implications of Hazardous Waste Determination}

It is only after the treatability study has been completed that the RCRA requirements for treatment, storage, and disposal facility operation in 40 CFR 261-270, including the LDRs in 40 CFR 268, may become an issue for either the testing facility or the sample originator. In accordance with the provisions of 40 CFR 261.4(f)(10), unused samples and treatability study residues must be evaluated by the testing facility to-determine whether the residues or unused samples are hazardous or the testing facility may return the samples and residues to the sample originator (40 CFR 261.4[f][10]). The criteria set forth in 40 CFR 261 would be used to determine whether the samples and residues were hazardous. If the testing facility returned the unused samples and residues to the sample originator, then the originator would become responsible for determining whether or not the samples and residues were hazardous in accordance with 40 CFR 262.11, "Hazardous Waste Determination." Samples and residues would then be defined as RCRA-regulated hazardous wastes and would need to be managed as such, including full compliance with the LDRs. The exemption from the generator requirements in 40 CFR 261.4(e) would no longer be applicable.

A determination must be made as to whether or not unused samples or residues are hazardous after the completion of a treatability study, whether the testing facility or the sample originator is making the determination. A characteristic hazardous waste may be rendered nonhazardous by the treatability study and therefore may be managed accordingly. A listed hazardous waste, although it would retain its hazardous status in accordance with 40 CFR 261.3, could conceivably be treated to a low enough concentration that it could be land disposed in accordance with the requirements of 40 CFR 268.

\subsubsection{Generator Requirements}

If treatability study samples are returned to the waste generator and a determination has been made that the samples are hazardous in accordance with 40 CFR 261.3, then the generator of the samples becomes a generator of hazardous waste and is subject to the requirements of 40 CFR 262, "Standards Applicable to Generators of Hazardous Waste." Among the generator requirements for the management of hazardous waste (including treatment, storage, transport, and disposal) are the following:

- The generator must obtain an identification number from the EPA.

- If the generator intends to transport hazardous waste for off-site treatment, storage, or disposal, a manifest must be prepared by the generator in accordance with the provisions of 40 CFR 262.20 , 262.21, 262.22, and 262.23.

- If the hazardous waste is to be transported under the circumstances described above, the generator must comply with requirements for packaging, labeling, placarding, and accumulation time in accordance with 40 CFR 262.30 through 262.34 . 
- The generator must comply with the recordkeeping requirements stipulated in 40 CFR 262.40 , "Recordkeeping." Requirements include the maintenance of copies of manifests, biennial reports and exception reports, test results, waste analyses, and required time periods for maintenance of records.

- Hazardous waste accumulation times must be observed in accordance with 40 CFR 262.34 (less than 90-day accumulation period).

In summary, DOE waste managers must be aware of the following issues when contracting for off-site treatability study services:

- The treatability study facility must have obtained an EPA Identification Number for treatability studies from either the state or the EPA region.

- The quantity limits are $1,000 \mathrm{~kg}$ of hazardous (nonacute) waste, $10,000 \mathrm{~kg}$ of media contaminated with nonacute hazardous waste, $1 \mathrm{~kg}$ of acute hazardous waste, or $2,500 \mathrm{~kg}$ of media contaminated with acute hazardous waste for each treatment process being evaluated for each generated waste stream.

- The daily throughput of the treatability study system can be no more than $10,000 \mathrm{~kg}$ of as-received media contaminated with nonacute hazardous waste, $2,500 \mathrm{~kg}$ of media contaminated with acute hazardous waste, or $250 \mathrm{~kg}$ of other as-received hazardous waste.

- The storage limit for any facility equals the daily throughput.

- If treatability study residues are returned to the generator, the generator must determine if the residues are hazardous in accordance with 40 CFR 261.3 and must dispose of them appropriately.

The facility must maintain records and manage residues according to applicable requirements. The EPA Regional Administrator must be notified in writing by the treatability study facility of the intent to conduct a treatability study no less than 45 days before conducting the treatability study.

\subsection{Toxic Substances Control Act}

\subsubsection{Applicability to Research and Development Facilities}

Treatability studies are not specifically addressed under TSCA. Further, the issue of what constitutes a research and development facility in accordance with TSCA is not clearly defined. A definition for "small quantities for research and development" can be found in 40 CFR 761.3 in reference to PCBs, and the language implies that laboratory testing qualifies as research and development. Therefore, treatability studies have been addressed under TSCA within the context of laboratory testing in a research and development facility.

\subsubsection{Exemptions for Research and Development Facilities}


Under 40 CFR 761.65(I), a laboratory that holds samples for eventual disposal is exempt from the notification and approval requirements for commercial storers. The sample is exempt from manifesting requirements if it is being transported to a laboratory for testing or back to the sample originator after testing (applicable DOT or U.S. Postal Service regulations do apply). However, TSCA distinguishes between samples sent to an analytical laboratory for the purpose of determining $\mathrm{PCB}$ concentrations and samples sent to research and development facilities to determine if a disposal method is suited for the waste (EPA, 1991a). The latter situation is interpreted by the EPA to include treatability study samples. Only samples sent to laboratories for determination of $\mathrm{PCB}$ concentrations may be exempt from the notification and manifesting requirements. Other types of samples, including treatability study samples, are subject to notification and manifesting requirements. Violation of proper manifesting procedures could result in enforcement action not only for the generator, but also the transporter, and possibly to the research and development facility if an Unmanifested Waste Report (40 CFR 761.211) is not completed.

Under 40 CFR 761.65(j), states and the federal government are exempt from the requirements of closure cost estimates and financial assurance for closure that are otherwise applicable to commercial storers of PCBs. PCB activities that are non-totally enclosed may be authorized by provisions under 40 CFR 761.30. While 40 CFR 761.20 ("Prohibitions") Subpart B ("Manufacturing, Processing, Distribution in Commerce and Use of PCBs and PCB Items") describes prohibitions against using PCBs or PCB items in any manner other than totally enclosed (except as indicated otherwise), 40 CFR 761.30(j) states that PCBs may be used in small quantities for research and development in a manner other than totally enclosed.

The DOE should be aware that the EPA is taking a position against shipping PCB-contaminated waste back to the sample originator after the completion of a treatability study. This position appears to contradict the provisions of RCRA, TSCA, and DOE Order 5820.2A. However, the EPA's position on this subject was discussed in two different letters from the EPA Office of Prevention, Pesticides, and Toxic Substances (EPA, 1995a; EPA, 1995b). The EPA believes that the only way to ensure proper management and disposal of PCBcontaminated waste is to require the permitee (the research and development facility) to assume responsibility for waste disposal. The EPA indicates that it may deviate somewhat on this policy on a case-by-case basis. Examples whereby a PCB waste may be returned to the originator include the originator agreeing to keep the returned waste separate and distinct from other wastes until disposal, a legal document that mandates the retum of the waste to the originator, or the waste having been treated to below 2 parts per million (ppm) for nonaqueous media and 3 parts per billion for aqueous media.

Since mixed waste containing PCBs presents some unique management issues, the DOE may wish to pursue an agreement involving the DOE, the EPA Office of Prevention, Pesticides, and Toxic Substances, and the treatability study facility. The agreement can stipulate whether or not the waste will be returned to the originator and how it will be managed. 


\subsubsection{TSCA Permits}

Permits, at least as they are known in the RCRA arena, are not discussed in the TSCA regulations. However, the EPA still requires that a permit to store PCB waste be obtained from the PCB Regional Coordinator representing the particular EPA region in which $\mathrm{PCB}$ waste storage is desired. The requirements for the permit are to be coordinated at the regional level. Other TSCA-related activities may also require permits, including permits for alternate disposal and treatment methods solicited from the PCB Regional Coordinator and a permit for a research and development facility. This permit stipulates specific conditions under which the facility may operate. TSCA-regulated activities that are conducted in more than one region require permitting from the Director, Exposure Evaluation Division.

Research and development into PCB disposal methods using a total of less than 500 pounds of PCB material, regardless of PCB concentration, will be reviewed and approved by the appropriate Regional EPA Administrator. Research and development efforts involving 500 pounds or more of PCB material will be reviewed and approved by the Director, Exposure Evaluation Division.

It should be noted that storage areas at RCRA-permitted facilities may be exempt from separate TSCA storage permitting if it can be demonstrated to the EPA Regional Administrator's satisfaction that the facility's existing RCRA closure plan is substantially equivalent to the closure plan standards of 40 CFR $761.65(\mathrm{e})$.

\subsubsection{Manifesting Issues}

In accordance with 40 CFR 761.65(1)(4), manifesting of samples for disposal must be performed by either the testing facility or by the sample originator if the sample is returned to the sample originator after testing. Manifest requirements for off-site storage or off-site disposal are summarized in 40 CFR 761.207 and 761.208. The requirements include stipulations for the identification of the type of PCB waste, weight in kilograms, container identification number or serial number for a PCB article, and designation of off-site facility to receive the waste, as well as specific procedures regarding use of the manifest.

\subsubsection{Disposal Considerations}

PCB treatability study samples become subject to PCB disposal regulations once research and development efforts on PCB samples have been concluded. 40 CFR 761, Subpart D, describes disposal requirements, as well as requirements for storage for disposal. In general, $\mathrm{PCBs}$ with a concentration of $50 \mathrm{ppm}$ or greater must be disposed of in an incinerator, in compliance with the provisions of $40 \mathrm{CFR}$ 761.70. Under $40 \mathrm{CFR}$ 761.60(e), requests for alternate methods of PCB destruction for PCBs that are required to be incinerated as a means of disposal that will result in a level of performance equivalent to the provisions of $40 \mathrm{CFR} 761.70$ may be submitted to the Administrator for consideration. The applicable Regional EPA Administrator will 
be contacted for alternate methods to be utilized in one EPA region only. The Director, Exposure Evaluation Division, will be contacted to request alternate disposal methods that will be used in more than one region.

Liquid PCBs at a concentration greater than $50 \mathrm{ppm}$ but less than $500 \mathrm{ppm}$ may be disposed of in an incinerator, a chemical waste landfill that complies with the requirements of $40 \mathrm{CFR} 761.75$, or a highefficiency boiler, according to 40 CFR 761.60. Nonliquid PCBs at a concentration greater than $50 \mathrm{ppm}$ in the form of contaminated soil, rags, or debris may be disposed of in an incinerator or a chemical waste landfill that complies with 40 CFR 761.75 .

\subsubsection{TSCA Versus RCRA}

PCBs are regulated not only under TSCA but are also referenced under RCRA in 40 CFR 261.8 and in the LDRs, 40 CFR 268.42 (liquid PCB waste concentrations greater than 50 ppm). A medium such as water, soil, debris, etc., contaminated only with PCBs is not a hazardous waste under RCRA unless it is hazardous by virtue of meeting the criteria for being characteristic per 40 CFR 261.21 through 261.24 or 261.31 through 261.33. If it can be determined that the PCB waste is characteristically hazardous, then the next step is to refer to 40 CFR 261.8, to determine if this waste can be excluded from regulation under RCRA, which provides for the exclusion of dielectric fluids containing PCBs if they are hazardous only because they fail toxicity characteristic leaching procedure levels for D018 through D043 constituents. PCB waste that meets this criteria would be regulated under TSCA, not RCRA. It should be noted that where RCRA authority has been granted to states, the DOE will need to communicate with state regulatory authorities concerning RCRA issues. However, states have not been granted TSCA authority. TSCA issues are handled by the PCB Regional Coordinator representing the particular EPA region in which a particular DOE facility or treatability study facility is located.

Assuming that the PCB waste still meets the criteria for being regulated under RCRA, then the LDRs become an issue. Under the RCRA LDRs, liquid PCB wastes with a PCB concentration greater than $50 \mathrm{ppm}$ are prohibited from land disposal. Referring back to the TSCA regulations, 40 CFR 761.1(e) states that the PCB regulations in Part 761 do not preempt other more stringent federal statutes and regulations. Therefore, although 40 CFR 761.60 states that liquid PCB wastes may be land disposed in a chemical waste landfill, it is possible that the EPA Regional Administrator will take the more stringent RCRA position that all PCB waste will be incinerated or otherwise thermally treated. The DOE should be prepared to discuss disposal options with the PCB Regional Coordinator and also the EPA Regional Administrator if the LDRs are applicable.

In summary, the TSCA regulations do not specifically identify or regulate treatability studies, but the following issues should be understood: 
- Small quantities of PCB samples can be used in research and development and are exempt from certain TSCA requirements such as totally enclosed systems, but they are not exempt from notification and manifesting requirements.

- Storage facilities in accordance with RCRA requirements may be acceptable for TSCA wastes, but the EPA Regional Administrator's approval is required.

- The EPA generally requires that research and development facilities assume responsibility for final waste disposition, although the EPA may be receptive to negotiating this issue if the waste containing PCBs also contains hazardous and radioactive constituents.

- Research and development activities on less than 500 pounds of PCB material require an approval by the EPA Regional Administrator.

- If PCB-contaminated waste is returned to the originator and the originator desires an alternate method of PCB disposal other than incineration, an approval must be obtained from the EPA Regional Administrator if only one EPA region is involved, or from the Administrator at the Headquarters level if more than one EPA region is involved.

- RCRA requirements apply in some situations, and the interaction should be carefully reviewed.

\subsection{CERCLA Off-Site Rule}

CERCLA authority extends beyond closed or abandoned facilities. If a facility owner/operator fails to implement RCRA due to recalcitrance or bankruptcy on the part of the owner/operator, or if there is an immediate environmental threat, CERCLA authority may prevail.

In accordance with 40 CFR 300.440, "Procedures for Planning and Implementing Off-Site Response Actions," off-site response actions refer to remedial or removal actions involving the off-site transfer of hazardous substances, pollutants, or contaminants as defined under CERCLA sections 101(14) and (33) that are fundfinanced or taken pursuant to CERCLA authority, or jointly between CERCLA and another authority. The off-site rule stipulates provision for the acceptance of CERCLA materials at off-site locations, and other provisions as applicable. Off-site transfers of the following laboratory samples and treatability study samples from CERCLA sites are exempt from the requirements of the off-site rule: (1) RCRA hazardous waste being transported from a CERCLA site for treatability studies and that also meet the exemption for treatability study waste specified in 40 CFR 261.4(e); (2) non-RCRA wastes transferred from a CERCLA site for treatability studies and that are below the quantity threshold established in 40 CFR 261.4(e)(2); and (3) samples of CERCLA wastes sent to a laboratory for characterization. Waste samples or residues may not be returned to the CERCLA site unless the remedial project manager or on-site coordinator gives approval and assures their proper management. 


\subsection{Nuclear Regulatory Commission Summary}

The NRC was established by the Energy Reorganization Act of 1974, as amended, which abolished the Atomic Energy Commission. It also transferred to the $\mathrm{NRC}$ all the licensing and related regulatory functions assigned to the Atomic Energy Commission by the Atomic Energy Act of 1954, as amended (10 CFR 1.1). In general, the DOE is self-regulating and therefore is not subject to NRC requirements with respect to management of radioactive waste and nuclear facilities and nuclear constituents. DOE policy makes off-site shipments to NRC-licensed facilities subject to NRC packaging and transportation requirements. Any non-DOE commercial facility that performs treatability studies on DOE mixed wastes is subject to the NRC requirements.

\subsubsection{Commercial Treatability Study Facility License Requirements}

The type of NRC license required for a commercial treatability study facility is dependent upon the type of radioactive material contained in the mixed-waste treatability study sample. For example, the licensing requirements of $10 \mathrm{CFR} 30,31$, and 33 would apply to treatability study facilities accepting mixed waste containing by-product material. The licensing requirements of $10 \mathrm{CFR} 40$ would apply to treatability study facilities accepting mixed waste containing source material. The licensing requirements of $10 \mathrm{CFR} 70$ would apply to treatability study facilities accepting mixed waste containing special nuclear material.

10 CFR 20 identifies the radiation protection standards that apply to treatability study facilities accepting mixed-waste samples and holding an NRC license. Treatability study facilities accepting mixed-waste samples must establish and maintain the following programs (10 CFR 20):

- A radiation protection program commensurate with the scope and extent of licensed activities in compliance with 10 CFR 20, Subpart B

- A program to monitor and comply with occupational dose limits in compliance with 10 CFR 20, Subpart C

- A program to monitor and comply with radiation dose limits for individual members of the public in compliance with 10 CFR 20, Subpart D

- Radiation survey and monitoring programs in compliance with 10 CFR 20, Subpart F

- A program to control the exposure from external sources in restricted areas in compliance with 10 CFR 20, Subpart G

- Respiratory protection and controls to restrict internal exposure in restricted areas in compliance with 10 CFR 20, Subpart H

- Storage and control programs for licensed materials in compliance with 10 CFR 20, Subpart I 
- Precautionary procedures in compliance with 10 CFR 20, Subpart J

- A waste disposal program in compliance with 10 CFR 20, Subpart $\mathrm{K}$

- A records management system in compliance with 10 CFR 20, Subpart L

- A reporting system in compliance with 10 CFR 20, Subpart M.

DOE waste managers contracting for treatability study services should ensure that the facility has these programs in place.

\subsubsection{Packaging and Transportation of Radioactive Material}

The requirements for packing, preparation for shipment, and transportation of licensed material, and the procedures and standards for NRC approval of packaging material are established in 10 CFR 71 . The packaging and transportation requirements of licensed material are also subject to $10 \mathrm{CFR} 20,21,30,39,40$, 70 , and 73, and to the regulations of the DOT and the U.S. Postal Service. A brief summary of DOT requirements is provided in Section 3.5.

Requirements to consider when preparing a mixed-waste treatability study sample for transportation to a treatability study facility are listed below:

- General licenses are required to transport or to deliver to a carrier any NRC-licensed material in compliance with 10 CFR 71, Subpart C.

- Package approval applications must be completed and approved in compliance with 10 CFR 71, Subpart D.

- Packages must meet the approval standards listed in 10 CFR 71, Subparts E and F.

- The originating organization and transporter must have established operating controls and procedures in compliance with 10 CFR 71, Subpart G.

- The originating organization and transporter must have an established quality assurance program in compliance with 10 CFR 71, Subpart H.

In addition, transportation of special nuclear material must also comply with the requirements of $10 \mathrm{CFR} 73$, which apply to the establishment and maintenance of a physical protection system for special nuclear material in transit. Topics to be considered include planning and scheduling, export/import shipments, security organization, driver training, contingency and response plan procedures, transfer and storage of special nuclear material for domestic shipment, access control and procedures, test and maintenance programs, transportation 
modes (10 CFR 73.26), and notification requirements (10 CFR 73.27). $10 \mathrm{CFR} 74$ requires material control and accounting of special nuclear material. Individuals who receive special nuclear material must also comply with the requirements of $10 \mathrm{CFR} 75$.

The requirements related to the export of mixed-waste treatability study samples are complicated and must comply with 10 CFR 110 .

\subsection{U.S. Department of Transportation Summary and Guidance}

The DOT regulations listed in 49 CFR relate to the transportation of materials. The regulations applicable to the transportation of mixed-waste treatability study samples from an originating organization to a treatability study facility are listed in 49 CFR 171 through 180, which are summarized below and in Table 3-1. Most of these requirements relate to the method used by the originating organization to package the material for shipment. Other requirements are the responsibility of the transportation company and relate to how the material is physically moved from the originating organization to the treatability study facility. The originating organization is responsible for verifying that the transporter is qualified; there are no specific transportation requirements for the treatability study facility that will receive the mixed-waste treatability study sample. These regulations should be consulted for additional information.

The requirements of $49 \mathrm{CFR} 171$ relate to general information, regulations, and definitions for the transportation of hazardous materials. The requirements of 49 CFR 172 relate to the preparation of shipping papers; marking, labeling, and placarding packagings and transportation vehicles; emergency response information; and training requirements.

The requirements of 49 CFR 173 relate to the general requirements for shipments and packages for shippers. Some of the requirements relate to the preparation of hazardous materials for shipment (49 CFR 173, Subpart B) and the classification of materials and packaging requirements (49 CFR 173, Subparts C, D, E, F, G, and 1).

The requirements of 49 CFR 174, 175, 176, and 177 relate to the specific modes of transportation chosen and should be consulted by the originating organization prior to shipment of the mixed-waste treatability study sample to the treatability study facility.

The requirements of $49 \mathrm{CFR} 178$ provide the specifications for packaging, including the manufacturing and testing specifications for packaging and containers used for the transportation of hazardous materials in commerce. The requirements of $49 \mathrm{CFR} 179$ provide the specifications for tank cars that are to be mounted on or from part of a tank car, and which are used for the transportation of hazardous materials in commerce. The requirements of $49 \mathrm{CFR} 180$ relate to the continuing qualification and maintenance of packaging. The requirements of 40 CFR 178,179 , and 180 are the responsibility of the originating organization. 
Table 3-1

U.S. Department of Transportation Requirements Applicable to Mixed-Waste Treatability Study Samples

\begin{tabular}{||l|l||}
\hline \multicolumn{1}{|c|}{ Topic } & \multicolumn{1}{c|}{ Applicable Regulation } \\
\hline \hline Packaging & 49 CFR 173, Subparts A and B and 173.401 through 173.478 \\
\hline Marking and labeling & $\begin{array}{l}49 \text { CFR 172, Subpart D and 172.400 through 172.207; 172.436 } \\
\text { through 172.440 }\end{array}$ \\
\hline Placarding & 49 CFR 172.500 through 172.519; 172.556; Appendices B and C \\
\hline Monitoring & 49 CFR 172, Subpart C \\
\hline Accident Reporting & 49 CFR 171.15 and 171.16 \\
\hline Shipping Papers & 49 CFR 172 \\
\hline Training & 49 CFR 172, Subpart $H$ \\
\hline \hline & \\
\hline Additional Requirements for Modes of Transportation \\
\hline Rail & \\
\hline Air & 49 CFR 174, Subparts $A$ through $D$ and $K$ \\
\hline Vessel & 49 CFR 175, Subparts $A$ through $D$ and $M$ \\
\hline Public Highway & 49 CFR 176, Subparts $A$ through $D$ and $M$ \\
\hline \hline
\end{tabular}

$C F R=$ Code of Federal Regulations 
In general, the DOT requirements are complex, but DOE sites have shipping and receiving expertise that can support these activities. Those personnel should be consulted to ensure that packaging, marking, and labeling are in compliance and that licensed shippers are used.

\subsection{DOE Order 5820.2A}

DOE Order 5820.2A establishes policies, guidelines, and minimum requirements for management of radioactive and mixed waste at DOE facilities. Radioactive and mixed waste must be managed to ensure protection of human health and safety, DOE and contractor employees, and the environment. Generation, treatment, storage, transportation, and disposal of radioactive and mixed waste must minimize waste generation and comply with all applicable federal, state, and local environmental, safety, and health laws and regulations, as well as DOE requirements. Policies are also developed by waste type. Waste storage requirements, recordkeeping and reporting, and waste transport including packaging, labeling, marking, and manifests are all discussed. This DOE Order should be consulted prior to preparing a mixed-waste treatability study sample for shipment. In some instances, a readiness review may be appropriate prior to shipment of mixed-waste treatability study samples.

\subsection{National Environmental Policy Act}

In accordance with Volume 57 of the Federal Register (57 FR 15122), waste treatability study activities performed by the DOE must undergo NEPA review to determine if new NEPA documentation for a particular DOE activity is necessary or if the activity is captured under an existing NEPA document. Documentation of NEPA status is the responsibility of the organization wishing to conduct the action or categorical exclusion. This decision regarding the appropriate level and adequacy of coverage is made by the DOE NEPA Compliance Officer with authority over the DOE site desiring to perform the activity. 


\section{TREATABILITY STUDY MANAGEMENT ISSUES}

Mixed-waste management is particularly perplexing because of the multiple regulatory agencies associated with the combined radioactive and hazardous constituents of the waste. Although the treatability study exemption is well understood and widely applied by developers of hazardous waste treatment technologies, a limited number of firms have the capability and licenses for the management of mixed waste.

Conducting a treatability study in compliance with applicable regulations and guidance and in a manner that provides the desired data requires careful planning and coordination. The EPA published guidance for conducting treatability studies under CERCLA in October 1992 (EPA, 1992a). Specific guidance on three soil cleanup technologies was also published by the EPA (EPA 1992b; EPA 1991b; EPA 1991c). There has not been specific guidance for treatability studies under RCRA, but the CERCLA guidance is readily adapted to RCRA waste treatability study issues. Jolley, Morris, and Singh from Martin Marietta Energy Systems published a guidance document on conducting technology demonstrations in the DOE's Oak Ridge facilities (Jolley et al., 1991). This document provides a step-by-step discussion of the management actions to conduct either on-site or off-site technology demonstrations under the DOE management structure.

Since the referenced guidance already exists, only key mixed-waste treatability study management steps and issues are highlighted in this document, with liberal reference to original sources of information. Both the EPA treatability study document (EPA, 1992a) and the Oak Ridge guidance document (Jolley et al., 1991) include complete logic diagrams indicating treatability study management steps and decisions. A simplified logic diagram (Figure 4-1) has been developed for this document to highlight major treatability study actions and to facilitate discussion of the steps of treatability study conduct. The chapter subsections discussing each element on the logic diagram are indicated beside the block on Figure 4-1.

\subsection{Is a Treatability Study Needed?}

Clearly, the first question is whether a treatability study should be considered at all. Many treatability studies have been conducted by DOE facilities, and some have been reported in the literature. Identification of those treatability studies can determine if previous results provide data that meet the objectives of the proposed investigation. However, general experience suggests that almost any waste/technology combination is a candidate for demonstration. Even minor variations in chemical composition can influence the effectiveness of some treatment processes, and a simple treatability test can determine if there is a problem and remedy the problem. 




Figure 4-1

Treatability Study Management Issues 
In addition to the technical benefits of a treatability study, there can be valuable schedular benefits in demonstrating the advantages/disadvantages of technologies to stakeholders. Visual or quantitative analyses of the performance of a technology can be an integral part of community interaction programs.

\subsection{Develop Data Quality Objectives}

The obvious objective of a treatability study is demonstration of waste treatment meeting the process or waste form performance specified in the applicable regulations. However, as a project proceeds from simple feasibility testing to development of process design data, increasing levels of quality control data come into play. One definition of data quality objectives is "qualitative and quantitative statements that specify the quality of the data required to support decisions concerning remedy selection and implementation" (EPA, 1992b). Development of data quality objectives was addressed in detail in an EPA guidance document (EPA, 1987). The reader is referred to that guidance for a complete discussion of the process. Table 4-1 shows elements of a simple phased approach for development of data quality objectives.

Unquestionably, DOE's larger waste streams and expected environmental restoration wastes are candidates for treatability studies and application of data quality objectives. Even then, the level of quality control on the data must be graded according to whether the technology application is in the simple screening phase (can the technology meet the regulatory requirements?), the technology selection phase (is this the best technology for the application?), or the final phase of collection of process design data.

However, DOE's small waste streams present different issues for development of the objectives. Invoking complete sampling and analytical quality control protocols for remedy design studies for very small volume waste streams may be unwarranted. Analytical costs could easily exceed the potential benefit of the study. Alternatively, some sites have considered the use of small volume wastes as surrogates to demonstrate treatment for larger waste streams or expected future wastes. In this way, the cost of the analytical data is justified.

\subsection{Are Waste Characterization Data Adequate?}

Collection of all available characterization data on the waste stream is necessary to support both shipment and treatment of the waste. The characterization data should be reviewed against the possible uses, which include the following:

- 'Developing handling procedures

- Supporting shipping designations

- Providing information to meet the supplier's waste acceptance criteria

- Providing process information necessary for the specific treatment technology. 
Table 4-1

Summary of Three-Stage Data

Quality Objectives Development Process ${ }^{\mathrm{a}}$

\begin{tabular}{|l||}
\hline \multicolumn{1}{|c||}{ Stage 1} \\
\hline Identify data users (include stakeholder concerns). \\
\hline Consult data sources for applicable performance data. \\
\hline Identify available waste characterization data. \\
\hline Identify the treatability study objectives and goals. \\
\hline \hline \\
\hline Stage 2 \\
\hline Identify data uses. \\
characteristics). \\
\hline Identify data quality needs. \\
\hline Identify data quantity needs. \\
\hline Evaluate sampling and analysis options. \\
\hline Review precision, accuracy, representativeness, completeness, and comparability parameters. \\
\hline \hline
\end{tabular}

asource: EPA, 1992a. 
Appendix B of the EPA's "Guide for Conducting Treatability Studies Under CERCLA" (EPA, 1992a) provides a list of required characterization data for several treatment technologies. The lengthy lists in that document address physical and chemical characterization that is necessary to ensure proper treatment and to ensure that the resultant treatability data can be used to define treatment process acceptance limits.

\subsection{Develop Plan/Obtain Data}

If it is determined that the necessary waste characterization data are not available, then plans for obtaining the data must be developed. A formal sampling and analysis plan may be required to ensure that the data collected are representative and complete. Components of a sampling and analysis plan are described in numerous guidance documents, including two by the EPA (EPA 1991b; EPA 1991c).

\subsection{Are Commercial Treatability Study Providers Available?}

The list of suppliers presented in Chapter 2 can be reviewed to determine if suppliers have technologies that potentially satisfy the RCRA requirements and if they have capabilities for mixed waste. It is also important to identify whether the radioactive materials licenses are consistent with the radioactive waste types of the treatability study samples. Transuranic wastes are one example of those that will be difficult to demonstrate in commercial facilities, as few facilities provide the multiple confinement barriers required for these wastes.

\subsection{Develop Procurement}

There are many procurement scenarios for obtaining treatability study services. Several sites (and at least one DOE Field Office) have recently developed broad scope contracts to facilitate mixed-waste treatment, and the source of the waste is not specific to the site developing the contract. These national contracts may offer an alternative to a time-consuming competitive procurement. Two methods of identification of these national contracts are offered. For general information, the DOE Environmental Management web site is under development and may provide useful contacts. This information is found at http://www.em.doe.gov; then

select "crosscut" and then "privatization." Specific contract information may also be available from the local DOE field office.

Jolley et al. (1991) recommended that procurement packages for commercial demonstrations should include the following:

- Objectives of the treatability study

- All available characterization data

- Definition of specific tasks/phases in the treatability study 
- Definition of deliverables, including work plans, sampling and analysis plans, health and safety plans, and final reports

- Expected schedule.

An additional item of concern is the disposition of the treatability study residues.

\subsection{Award Contract}

An on-site review of the treatability study supplier's facilities is warranted to ensure that all required permits are in place and that practices and procedures indicate that the treatability study objectives will be met. Recommended items to review include the following:

- Specific permits, including RCRA, NRC, or state licenses; air permits; and discharge permits

- Inspection records of regulatory authorities

- Documentation of previous treatability studies done on the site, including all required records

- Radiation safety provisions

- Quality assurance/quality control procedures if on-site analytical facilities are used

- Posting in compliance with RCRA requirements.

\subsection{Ship Waste}

Chapter 3 of this document summarizes the general requirements for transport of treatability study samples. In summary, there are requirements for placarding, labeling, and packaging of the samples. Knowledgeable site personnel with expertise in the transportation of radioactive samples should be contacted to ensure all proper procedures are followed.

\subsection{Monitor Test}

It is recommended that at least one phase of the treatability study testing should be monitored to confirm that all elements of the sampling plan and the health and safety plan are being followed. It is particularly important to ensure compliance with test plans if the treatability study is being used to support technology selection or to develop design data. In these cases, a simple confirmation that tests were run according to plan is warranted should questions arise during community relations discussions.

Procedures should be established to facilitate rapid approval/disapproval of requested test plan changes that may arise during conduct of the test. Jolley et al. (1991) recommended assignment of responsible personnel for ready access by the treatability study supplier to address test plan changes. 


\subsection{Review Data}

Compliance with test objectives is best determined by review of the test data. The importance is amplified if the data will be used to introduce the technology to stakeholders or cognizant regulators. A data validation review is recommended, in addition to a quantitative check of parameters that confirm compliance with the test objectives.

\subsection{Disposition of Treatment Residuals}

Disposition of the residuals should be identified in the contract. Section 3.1.2 of this report discusses this issue in detail. Depending on the original regulatory status of the sample, the residues may be considered a new waste or simply the residue from treatment of a listed waste. It would also be prudent to ensure that secondary wastes are appropriately addressed by the supplier.

\subsection{File Report}

The treatability study report should become part of the project file. More significantly, the results should be reported at DOE meetings to ensure that all interested DOE sites have access to the data for possible use at those sites. Alternatively, distribution to the responsible technology development managers at each site would benefit the DOE complex. 


\section{CONCLUSIONS}

The DOE's mixed-waste streams are diverse and present unique management issues. Treatability studies may be utilized as an integral part of the DOE's mixed-waste management program. Commercial treatability study suppliers have been identified that either have current capability in their own facilities or have access to licensed facilities owned by others. Facilities owned by others may have access to a broader range of technologies, which would facilitate the mixed-waste management process.

Numerous federal and state regulations, as well as DOE Order 5820.2A, impact the performance of treatability studies. Generators, transporters, and treatability study facilities are subject to regulation. From a mixedwaste standpoint, a key requirement is that the treatability study facility must have an NRC or state license that allows it to possess radioactive materials. From a RCRA perspective, the facility must support treatability study activities with the applicable plans, reports, and documentation. If PCBs are present in the waste, TSCA will also be an issue. The requirements of CERCLA may apply, and both DOT and NRC regulations will impact the transportation of DOE mixed waste to an off-site treatment facility. DOE waste managers will need to be cognizant of all applicable regulations as mixed-waste treatability study programs are initiated. 


\section{REFERENCES}

EPA, see U.S. Environmental Protection Agency.

Federal Register, Vol. 57, pg. 15122, April 24, 1992.

Jolley, R. L., M. I. Morris, and S. P. N. Singh, 1991, "Guidance Manual for Conducting Technology Development Activities," ORNLTM-11848, Oak Ridge National Laboratory, Oak Ridge, Tennessee.

Smith, M., R. Britton, T. Kraus, and R. Mayes, 1995, "Review of Private Sector Treatment, Storage, and Disposal Capacity for Radioactive Waste," INEL-95/0020, Idaho National Engineering Laboratory, Idaho Falls, Idaho.

U.S. Environmental Protection Agency (EPA), 1995a, Letter from the EPA Office of Prevention, Pesticides, and Toxic Substances, Washington, D.C., October 17, 1995.

U.S. Environmental Protection Agency (EPA), 1995b, Letter from the EPA Office of Prevention, Pesticides, and Toxic Substances, Washington, D.C., July 19, 1995.

U.S. Environmental Protection Agency (EPA), 1994, "Vendor Information System for Innovative Treatment Technologies," Version 3.0, EPA 542-R-93-003, U.S. Environmental Protection Agency, Washington, D.C.

U.S. Environmental Protection Agency (EPA), 1992a, "Guide for Conducting Treatability Studies Under CERCLA," EPA.540R-92/07IA, U.S. Environmental Protection Agency, Office of Solid Waste and Emergency Response, Washington, D.C.

U.S. Environmental Protection Agency (EPA), 1992b, "Guide for Conducting Treatability Studies Under CERCLA: Solvent Extraction," EPA/540/R-92/016a, U.S. Environmental Protection Agency, Washington, D.C.

U.S. Environmental Protection Agency (EPA), 1991a, Memorandum from the Office of Pesticides and Toxic Substances, U.S. Environmental Protection Agency, Washington, D.C., December 17, 1991.

U.S. Environmental Protection Agency (EPA), 1991b, "Guide for Conducting Treatability Studies Under CERCLA: Soil Vapor Extraction," EPA/540/2-91/019a, U.S. Environmental Protection Agency, Washington, D.C.

U.S. Environmental Protection Agency (EPA), 1991c, "Guide for Conducting Treatability Studies Under CERCLA: Soil Washing," EPA/540/2-91/020a, U.S. Environmental Protection Agency, Washington, D.C.

U.S. Environmental Protection Agency (EPA), 1987, "Data Quality Objectives for Remedial Response Activities," EPA/540/g-87/003, U.S. Environmental Protection Agency, Washington, D.C. 
ATTACHMENT 1

MIXED WASTE TREATABILITY STUDY TECHNOLOGY QUESTIONNAIRE 
MIXED WASTE TREATABILITY STUDY TECHNOLOGY QUESTIONNAIRE

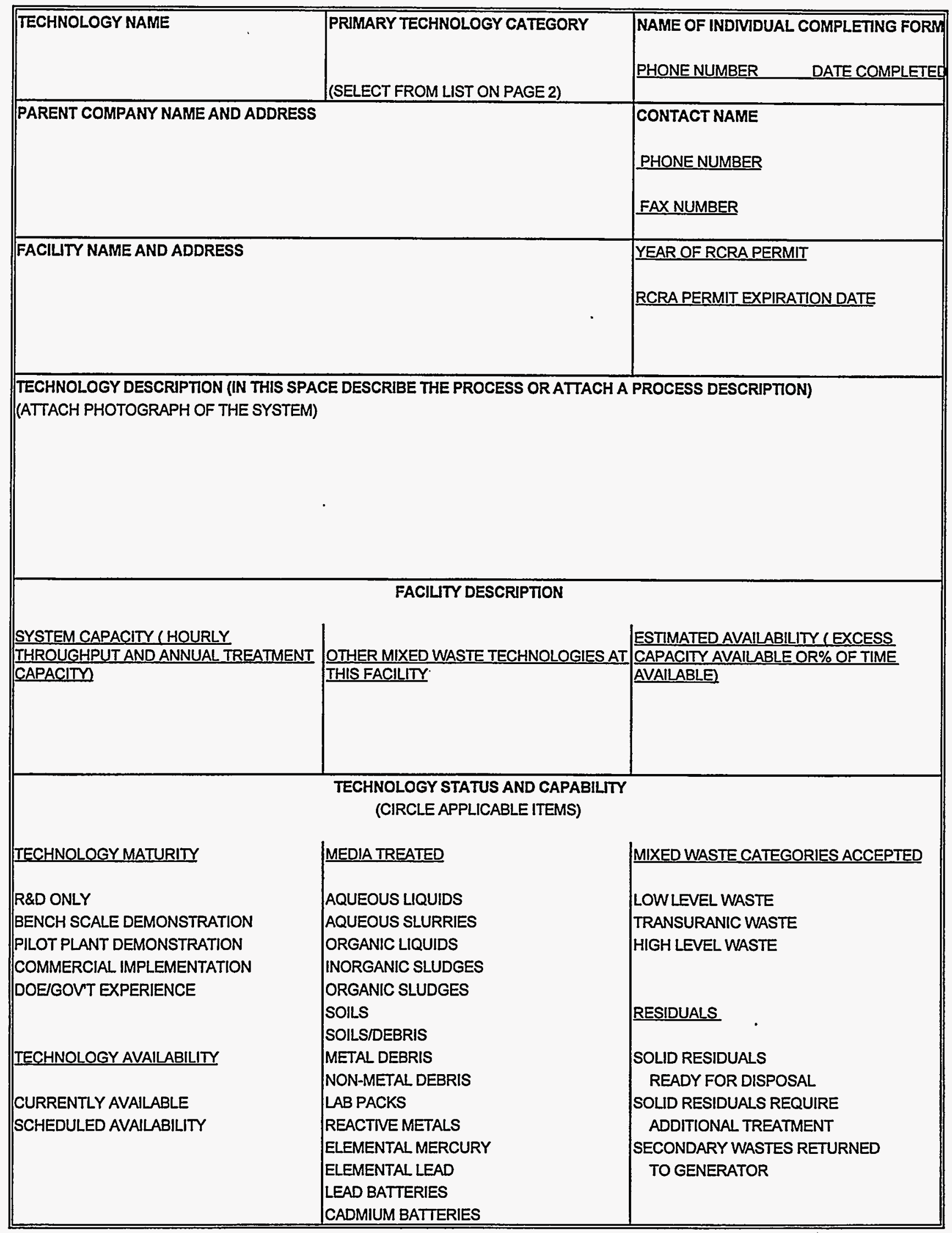


NOTE THAT INFORMATION SUPPLIED ON THIS PAGE IS FOR MIXED WASTE FOCUS AREA FILE INFORMATION ONLY

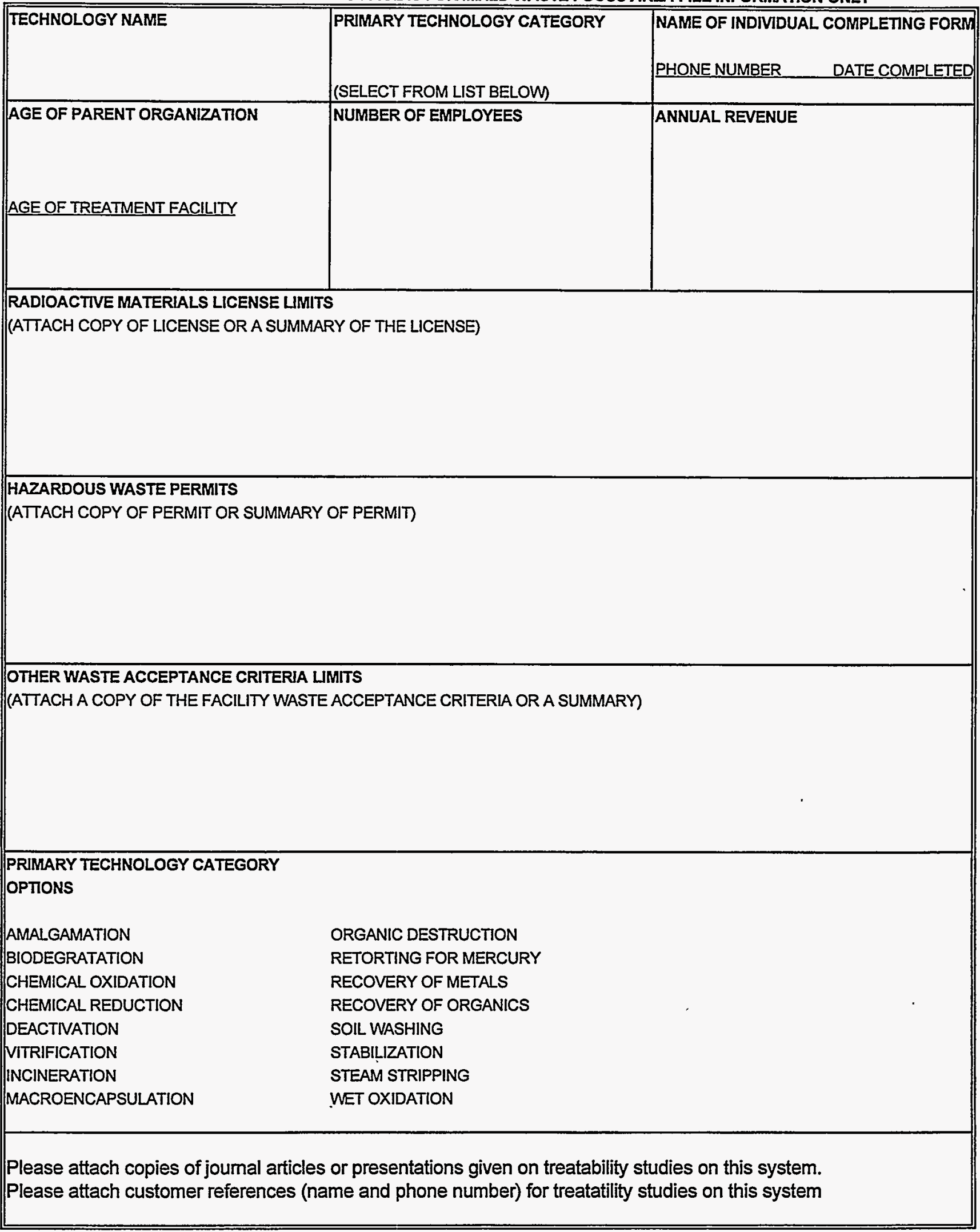

Page 2 


\section{ATTACHMENT 2}

\section{SUMMARIES OF TREATABILITY STUDY}

SUPPLIER QUESTIONNAIRE RESPONSES 
MIXED WASTE TREATABILITY STUDY TECHNOLOGY QUESTIONAIRE

\begin{tabular}{|c|c|c|}
\hline $\begin{array}{l}\text { Technology Name: } \\
\text { Soil Washing }\end{array}$ & $\begin{array}{l}\text { Technology Category: } \\
\text { Soil Washing }\end{array}$ & \\
\hline \multicolumn{2}{|l|}{$\begin{array}{l}\text { Company Name and Address: } \\
\text { Alternative Remedial Technologies } \\
14497 \text { North Dale Mabry Highway } \\
\text { Suite } 140 \\
\text { Tampa, FL } 33618\end{array}$} & $\begin{array}{l}\text { Contact: } \\
\text { Mr. Michael Mann } \\
\text { Phone: } \\
\text { (813) 264-3571 } \\
\text { Fax: } \\
\text { (813) } 962-0867\end{array}$ \\
\hline $\begin{array}{r}\text { Age of Company: } \\
108 \text { years }\end{array}$ & $\begin{array}{c}\text { Number of Employees: } \\
4,854 \\
\end{array}$ & $\begin{array}{r}\text { Annual Revenue: } \\
\$ 473 \text { million } \\
\end{array}$ \\
\hline \multirow{4}{*}{\multicolumn{2}{|c|}{$\begin{array}{l}\text { Facility Name and Address: } \\
\text { Mobile Treatment Facility }\end{array}$}} & Age of Facility: $\quad$ N/A \\
\hline & & RCRA Treatment Permit \\
\hline & & Date: $\quad$ Exp: \\
\hline & & Rad Mat \#: None \\
\hline \multicolumn{3}{|c|}{$\begin{array}{l}\text { Technology Description: } \\
\text { The ART/Heidimij soil washing system is based upon proven principles and equipment from the mining and mineral } \\
\text { processing businesses. The key to successful soil washing is partially in the arrangement and configuration of unit } \\
\text { processes, but more importantly in the characterization and understanding of the soil matrix/contaminant relationship. This } \\
\text { "know-how" is the most important attribute to the ART/Heidimij team. This approach to soil washing is simple: separate and } \\
\text { treat the oversize and sand fractions so that they may be placed back on the site as clean backfill, while concentrating the } \\
\text { contaminants in the fines for further treatment or disposal. }\end{array}$} \\
\hline $\begin{array}{l}\text { System } 25 \mathrm{tph} \\
\text { Capacity: }\end{array}$ & $\begin{array}{l}\text { Excess } \\
\text { Capacity: }\end{array}$ & $\begin{array}{l}\text { Availability is dependent upon current project } \\
\text { commitments. }\end{array}$ \\
\hline $\begin{array}{c}\text { Technology Maturity: } \\
\square \text { R and D } \\
\square \quad \text { Bench Scale } \\
\square \quad \text { Pilot Scale } \\
\square \quad \text { Commercial } \\
\text { Pick the best one }\end{array}$ & \multirow{4}{*}{$\begin{array}{l}\text { Media Treated: } \\
\square \text { Aqueous liquids } \\
\square \text { Aqueous slurries } \\
\square \text { Organic liquids } \\
\square \text { Inorganic sludges } \\
\square \text { Organic sludges } \\
\square \text { Soils } \\
\square \text { Soils and debris } \\
\square \text { Metal debris } \\
\square \text { Non-metal debris } \\
\square \text { Lab packs } \\
\square \text { Reactive metals } \\
\square \text { Elemental mercury } \\
\square \text { Elemental lead } \\
\square \text { Lead batteries } \\
\square \text { Cadmium batteries } \\
\text { Select all that apply }\end{array}$} & $\begin{array}{l}\text { MW Categories Accepted: } \\
\square \text { Low level waste } \\
\square \text { Transuranic waste } \\
\square \text { High level waste } \\
\text { Select all that apply }\end{array}$ \\
\hline$\square$ DOEJGov experience & & Residuals: \\
\hline Technology Availability: & & $\begin{array}{l}\text { Residuals ready tor } \\
\text { disposal }\end{array}$ \\
\hline $\begin{array}{l}\square \text { Currently available } \\
\square \text { Scheduled availability } \\
\text { Pick the best one } \\
\text { Scheduled Date: }\end{array}$ & & $\begin{array}{l}\square \text { Residuals need } \\
\text { additional treatment } \\
\square \text { Secondary wastes } \\
\text { returned to generator } \\
\text { Select all that apply }\end{array}$ \\
\hline
\end{tabular}


MIXED WASTE TREATABILITY STUDY TECHNOLOGY QUESTIONAIRE

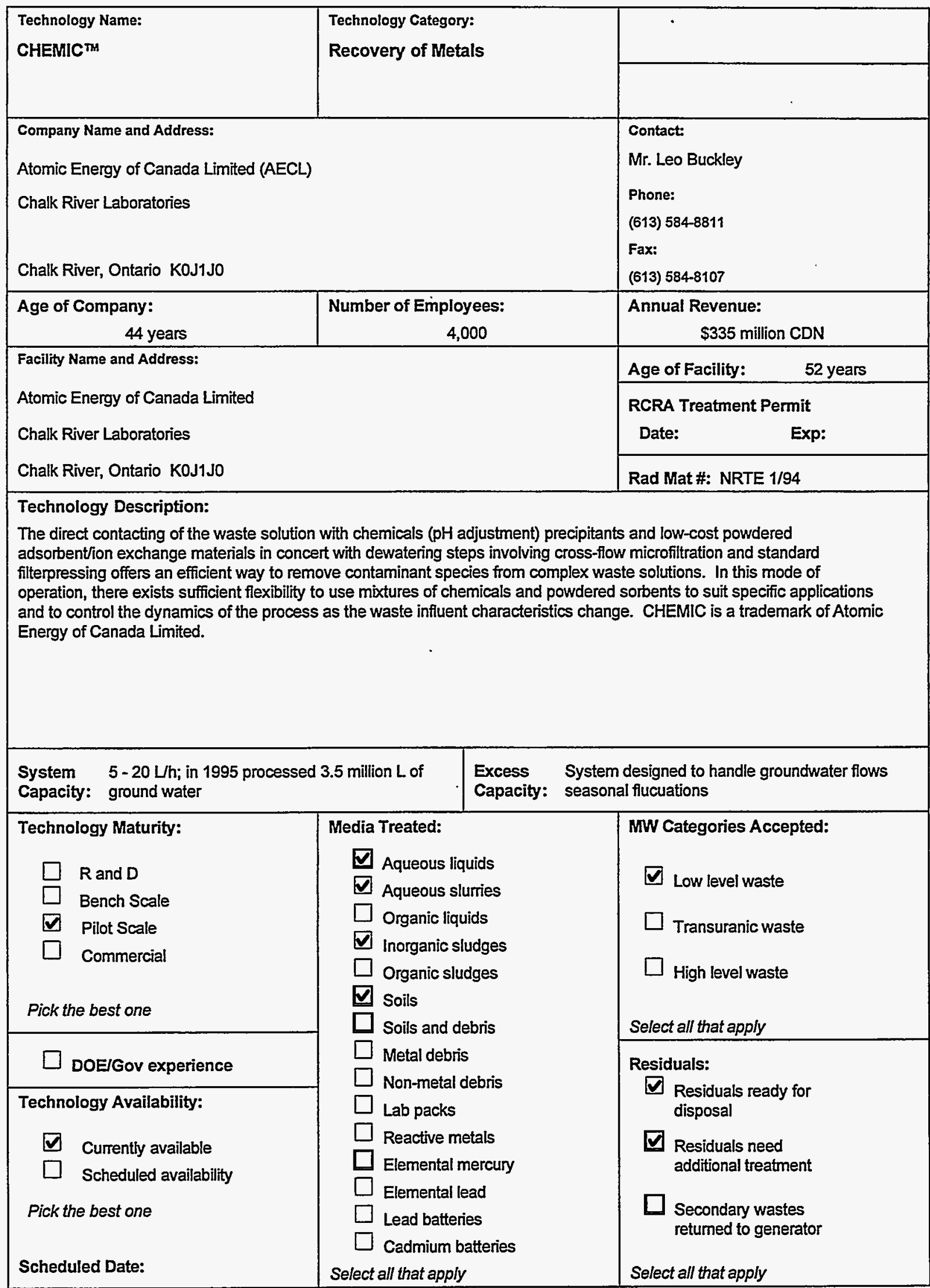


MIXED WASTE TREATABILITY STUDY TECHNOLOGY QUESTIONAIRE

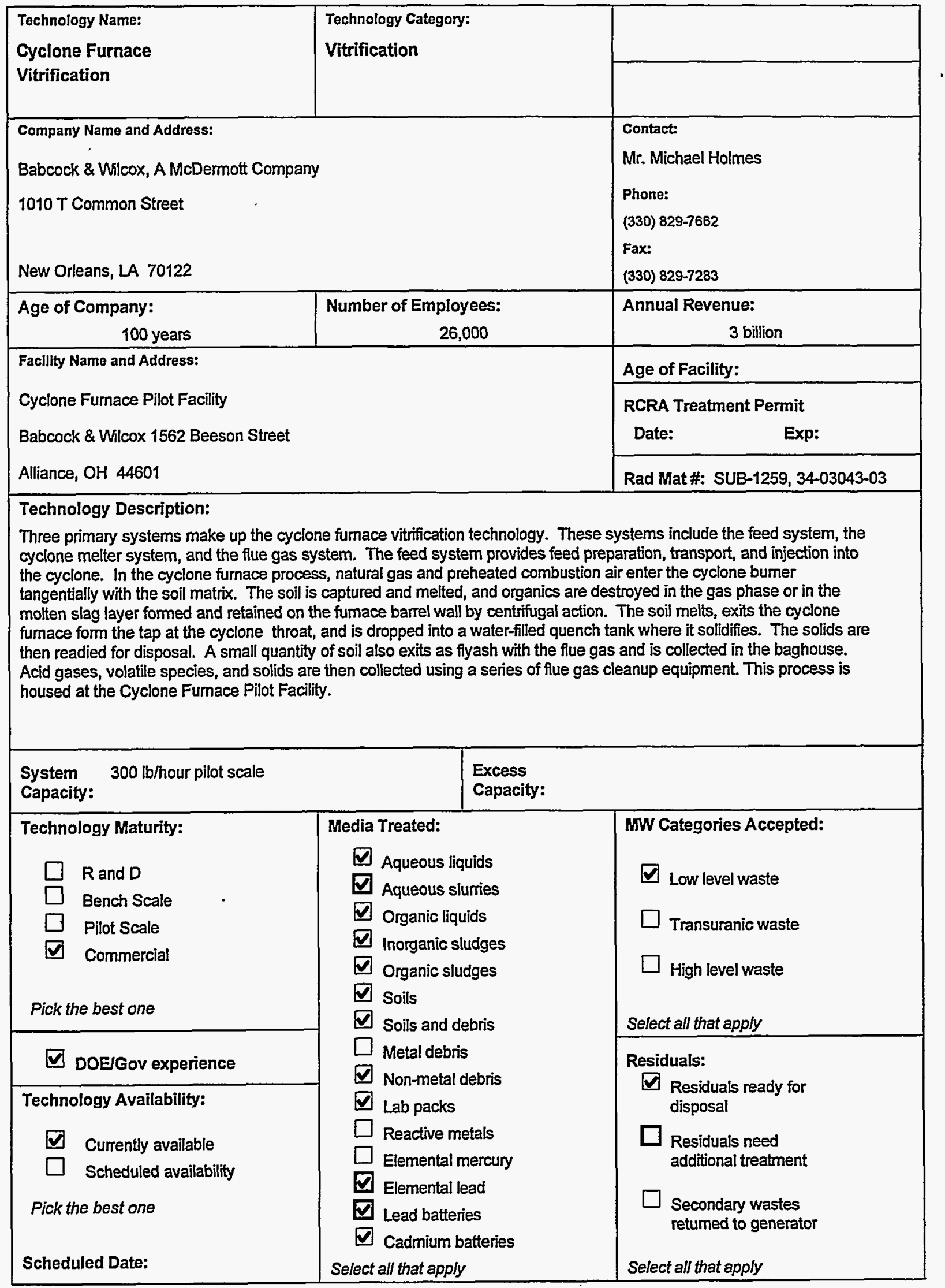




\section{MIXED WASTE TREATABILITY STUDY TECHNOLOGY QUESTIONAIRE}

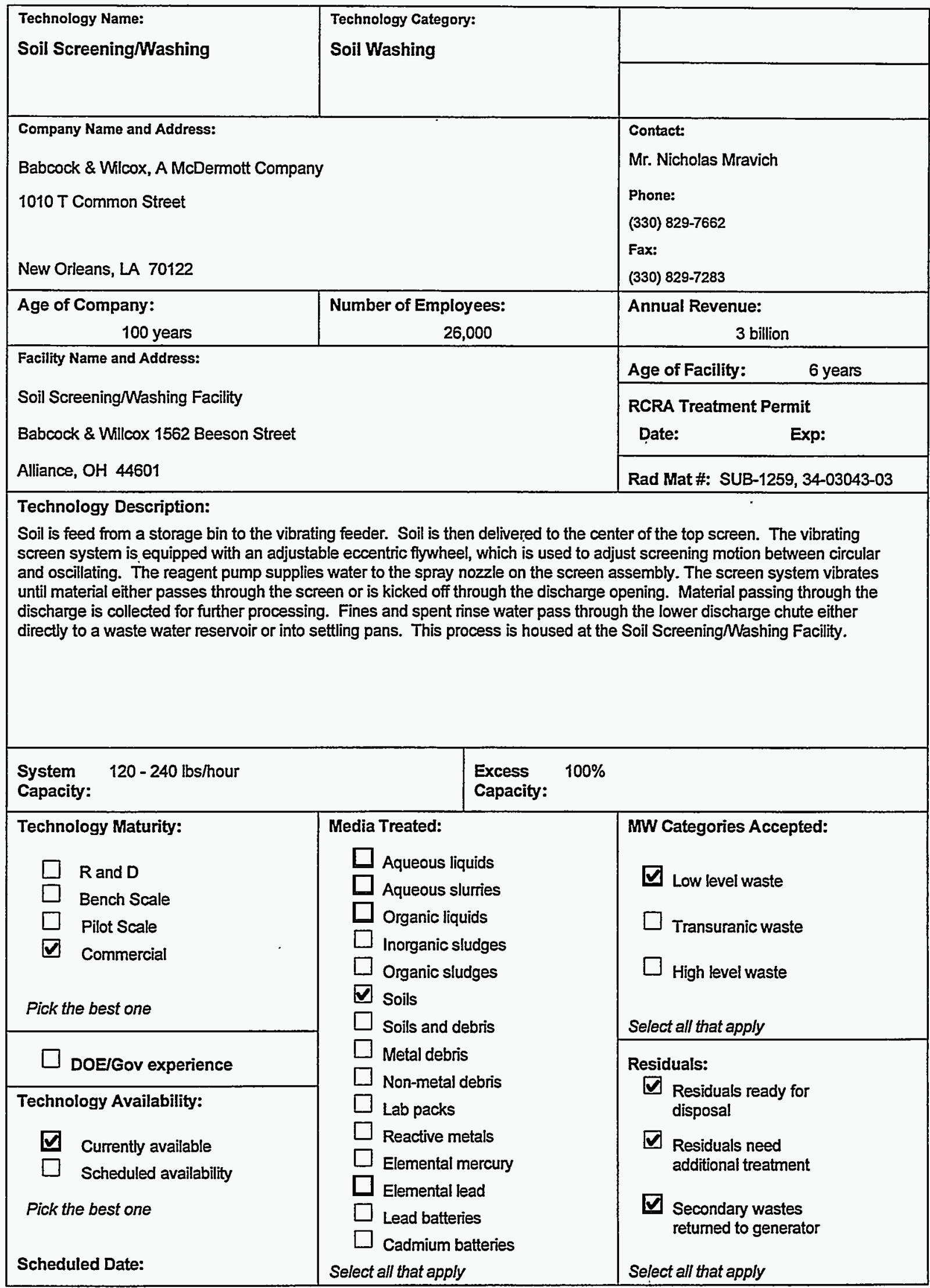


MIXED WASTE TREATABILITY STUDY TECHNOLOGY QUESTIONAIRE

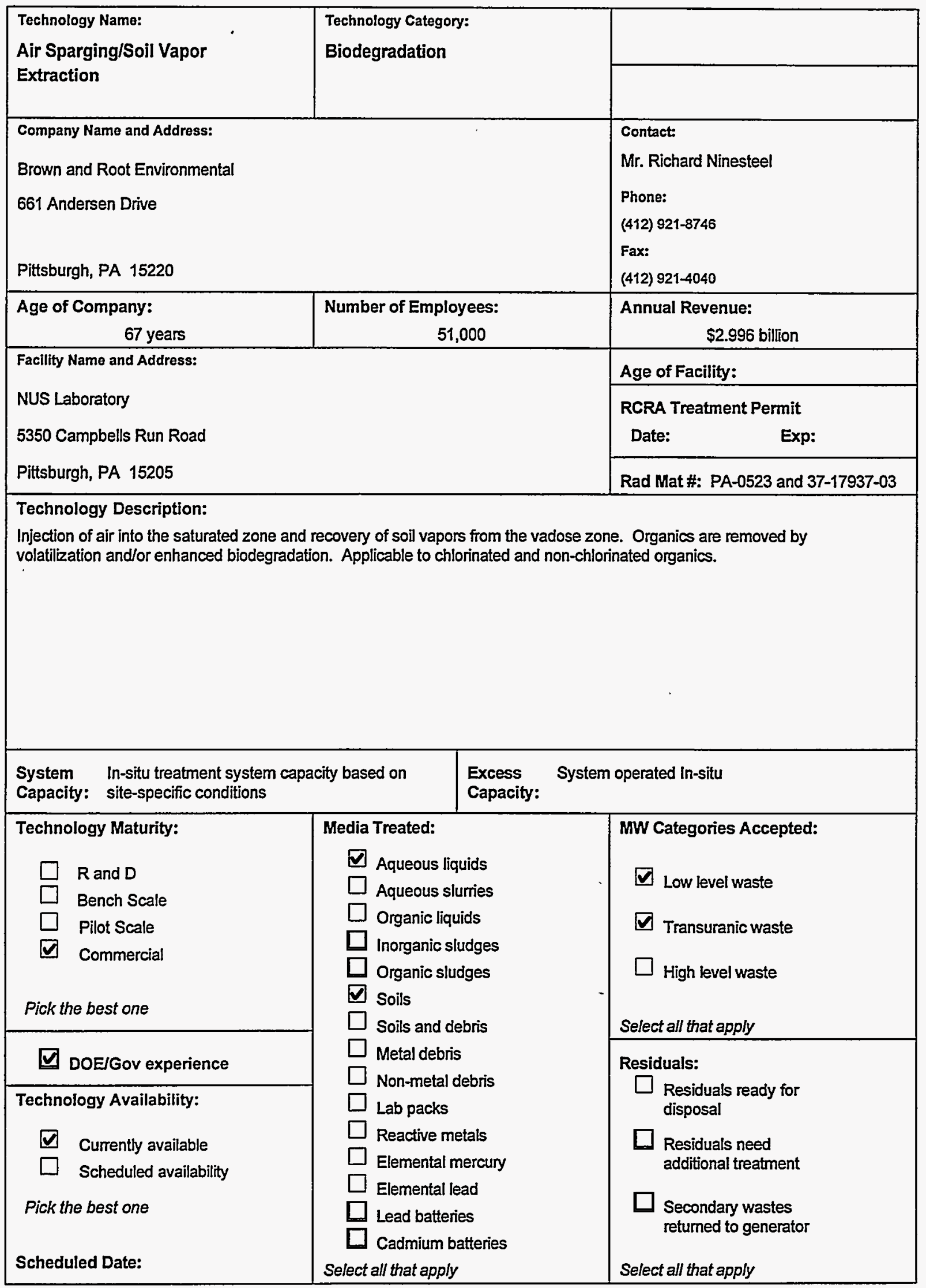


MIXED WASTE TREATABILITY STUDY TECHNOLOGY QUESTIONAIRE

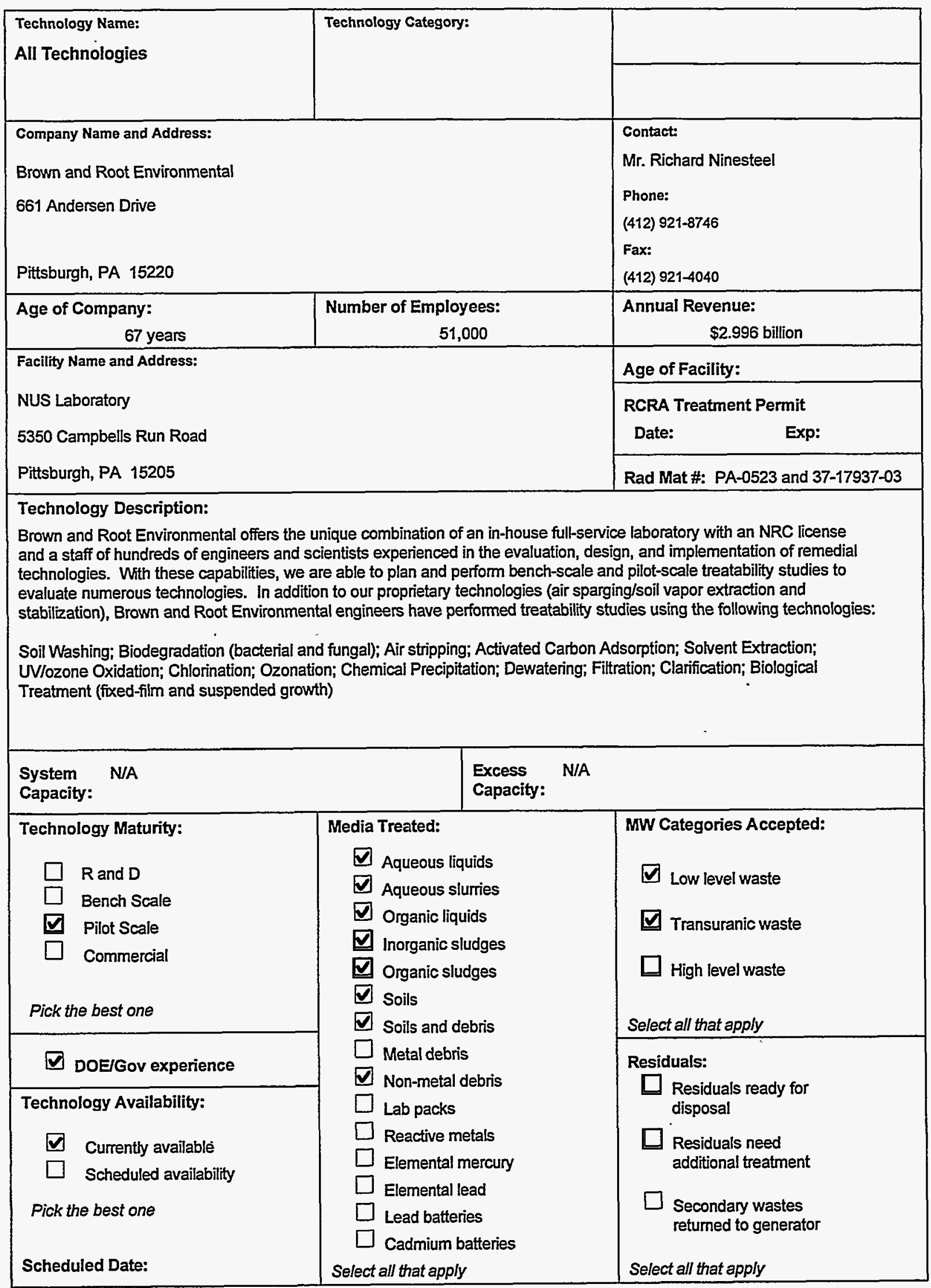


MIXED WASTE TREATABILITY STUDY TECHNOLOGY QUESTIONAIRE

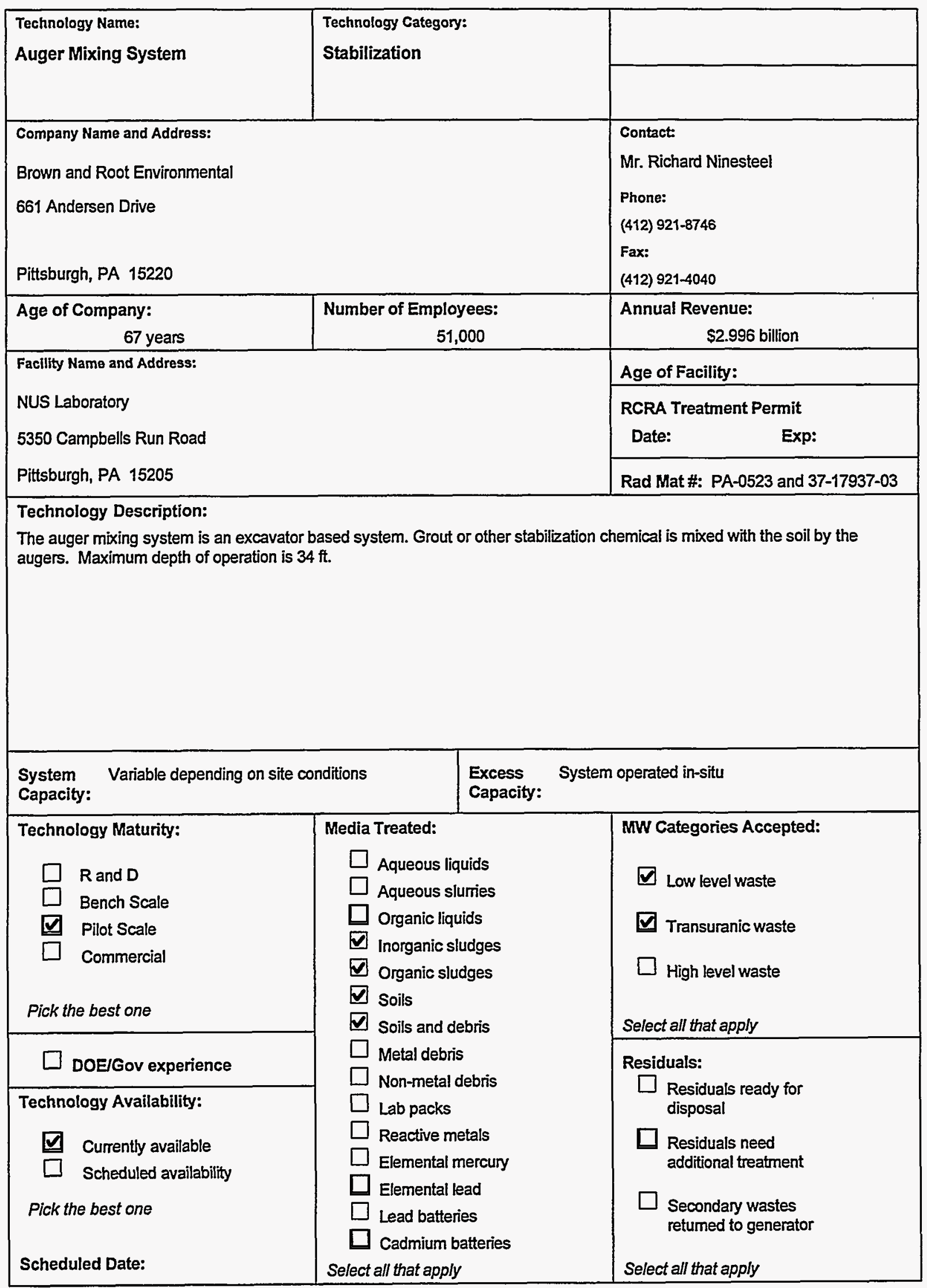




\section{MIXED WASTE TREATABILITY STUDY TECHNOLOGY QUESTIONAIRE}

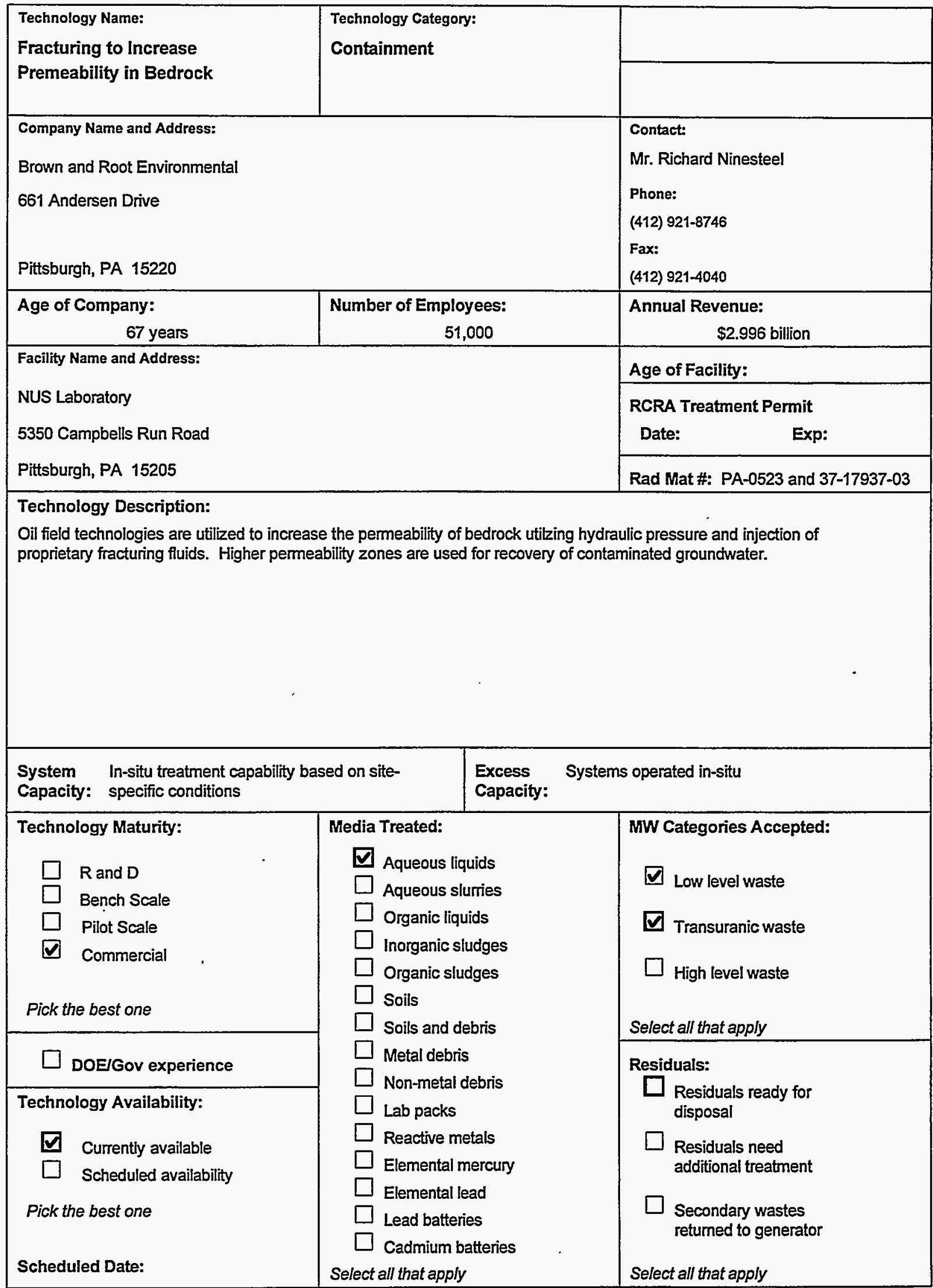


MIXED WASTE TREATABILITY STUDY TECHNOLOGY QUESTIONAIRE

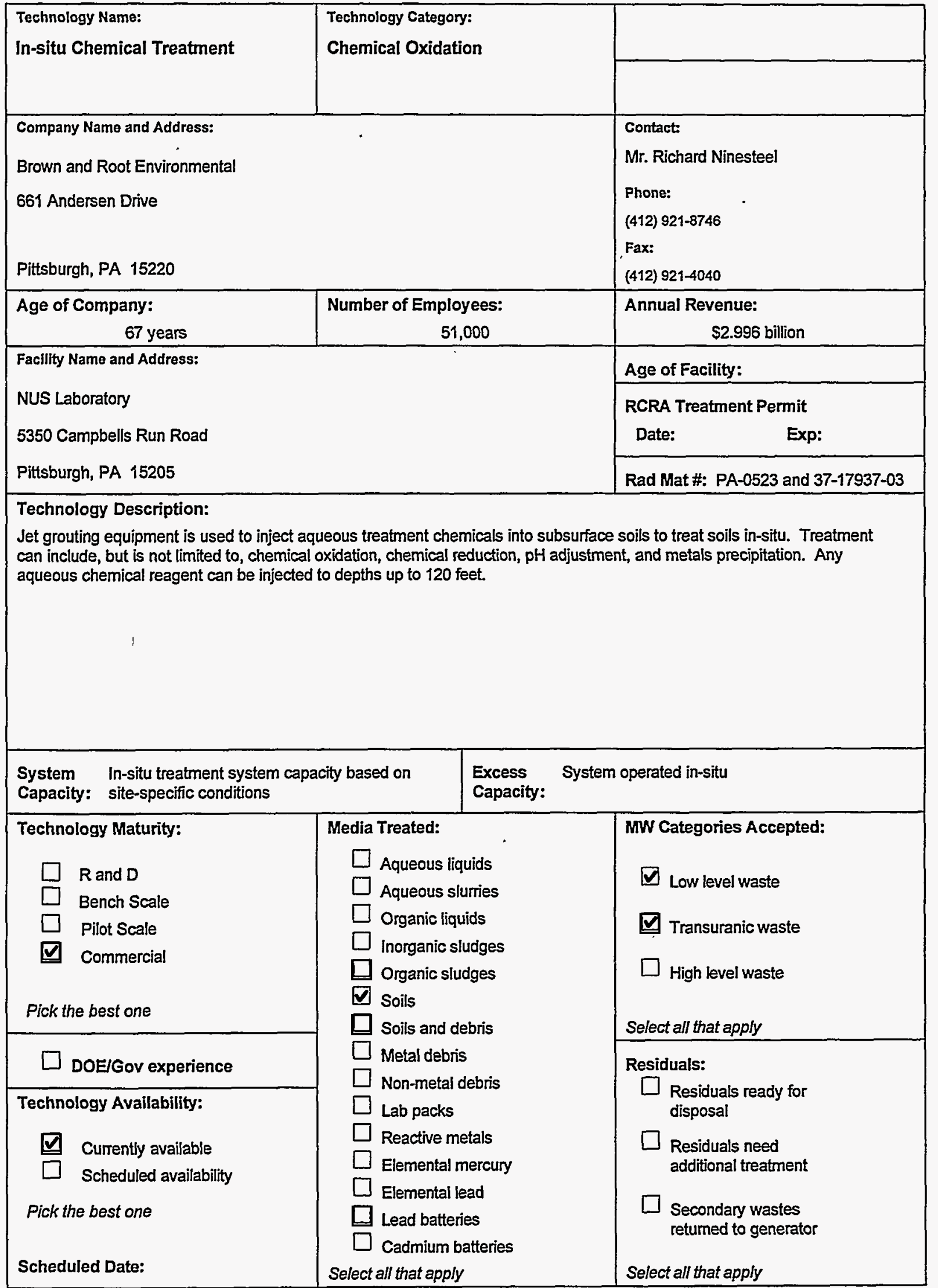


MIXED WASTE TREATABILITY STUDY TECHNOLOGY QUESTIONAIRE

\begin{tabular}{|c|c|c|c|}
\hline $\begin{array}{l}\text { Technology Name: } \\
\text { Jet Grouting }\end{array}$ & \multicolumn{2}{|c|}{$\begin{array}{l}\text { Technology Category: } \\
\text { Stabilization }\end{array}$} & \\
\hline \multicolumn{3}{|l|}{$\begin{array}{l}\text { Company Name and Address: } \\
\text { Brown and Root Environmental } \\
661 \text { Andersen Drive }\end{array}$} & $\begin{array}{l}\text { Contact: } \\
\text { Mr. Richard Ninesteel } \\
\text { Phone: } \\
\text { (412) } 921-8746 \\
\text { Fax: } \\
\text { (412) } 921-4040\end{array}$ \\
\hline $\begin{array}{l}\text { Age of Company: } \\
\qquad 67 \text { years }\end{array}$ & \multicolumn{2}{|c|}{$\begin{array}{l}\text { Number of Employees: } \\
\qquad 51,000 \\
\end{array}$} & $\begin{array}{l}\text { Annual Revenue: } \\
\qquad \$ 2.996 \text { billion }\end{array}$ \\
\hline \multirow{3}{*}{\multicolumn{3}{|c|}{$\begin{array}{l}\text { Facility Name and Address: } \\
\text { NUS Laboratory } \\
5350 \text { Campbells Run Road } \\
\text { Pittsburgh, PA } 15205\end{array}$}} & Age of Facility: \\
\hline & & & $\begin{array}{l}\text { RCRA Treatment Permit } \\
\text { Date: } \quad \text { Exp: }\end{array}$ \\
\hline & & & Rad Mat \#: PA-0523 and 37-17937-03 \\
\hline \multicolumn{4}{|c|}{$\begin{array}{l}\text { Technology Description: } \\
\text { Jet grouting mixes a cement siurry into the soil under high-energy conditions to produce a thorough in-situ mixture of soil } \\
\text { and cement, refered to as soil-cement or "soilcrete". Columns of } 24 \text { to } 48 \text { inches in diameter to depth up to } 175 \text { feet can be } \\
\text { created. Unlike slurry wall construction, jet grout wall construction does not require excavation. }\end{array}$} \\
\hline \multicolumn{2}{|c|}{$\begin{array}{l}\text { System Variable depending on site conditions } \\
\text { Capacity: }\end{array}$} & \multicolumn{2}{|c|}{$\begin{array}{l}\text { Excess System operated in-situ } \\
\text { Capacity: }\end{array}$} \\
\hline  & \multirow{3}{*}{\multicolumn{2}{|c|}{$\begin{array}{l}\text { Media Treated: } \\
\square \text { Aqueous liquids } \\
\square \text { Aqueous slurries } \\
\square \text { Organic liquids } \\
\square \text { Inorganic sludges } \\
\square \text { Organic sludges } \\
\square \text { Soils } \\
\square \text { Soils and debris } \\
\square \text { Metal debris } \\
\square \text { Non-metal debris } \\
\square \text { Lab packs } \\
\square \text { Reactive metals } \\
\square \text { Elemental mercury } \\
\square \text { Elemental lead } \\
\square \text { Lead batteries } \\
\square \text { Cadmium batteries } \\
\text { Select all that apply }\end{array}$}} & $\begin{array}{l}\text { MW Categories Accepted: } \\
\square \text { Low level waste } \\
\square \text { Transuranic waste } \\
\square \text { High level waste } \\
\text { Select all that apply }\end{array}$ \\
\hline$\square$ DOE/Gov experience & & & Residuals: \\
\hline $\begin{array}{l}\text { Technology Availability: } \\
\qquad \text { Currently available } \\
\square \text { Scheduled availability } \\
\text { Pick the best one } \\
\text { Scheduled Date: }\end{array}$ & & & $\begin{array}{l}\square \text { Residuals ready for } \\
\text { disposal } \\
\square \text { Residuals need } \\
\text { additional treatment } \\
\square \text { Secondary wastes } \\
\text { retumed to generator } \\
\text { Select all that apply }\end{array}$ \\
\hline
\end{tabular}


MIXED WASTE TREATABILITY STUDY TECHNOLOGY QUESTIONAIRE

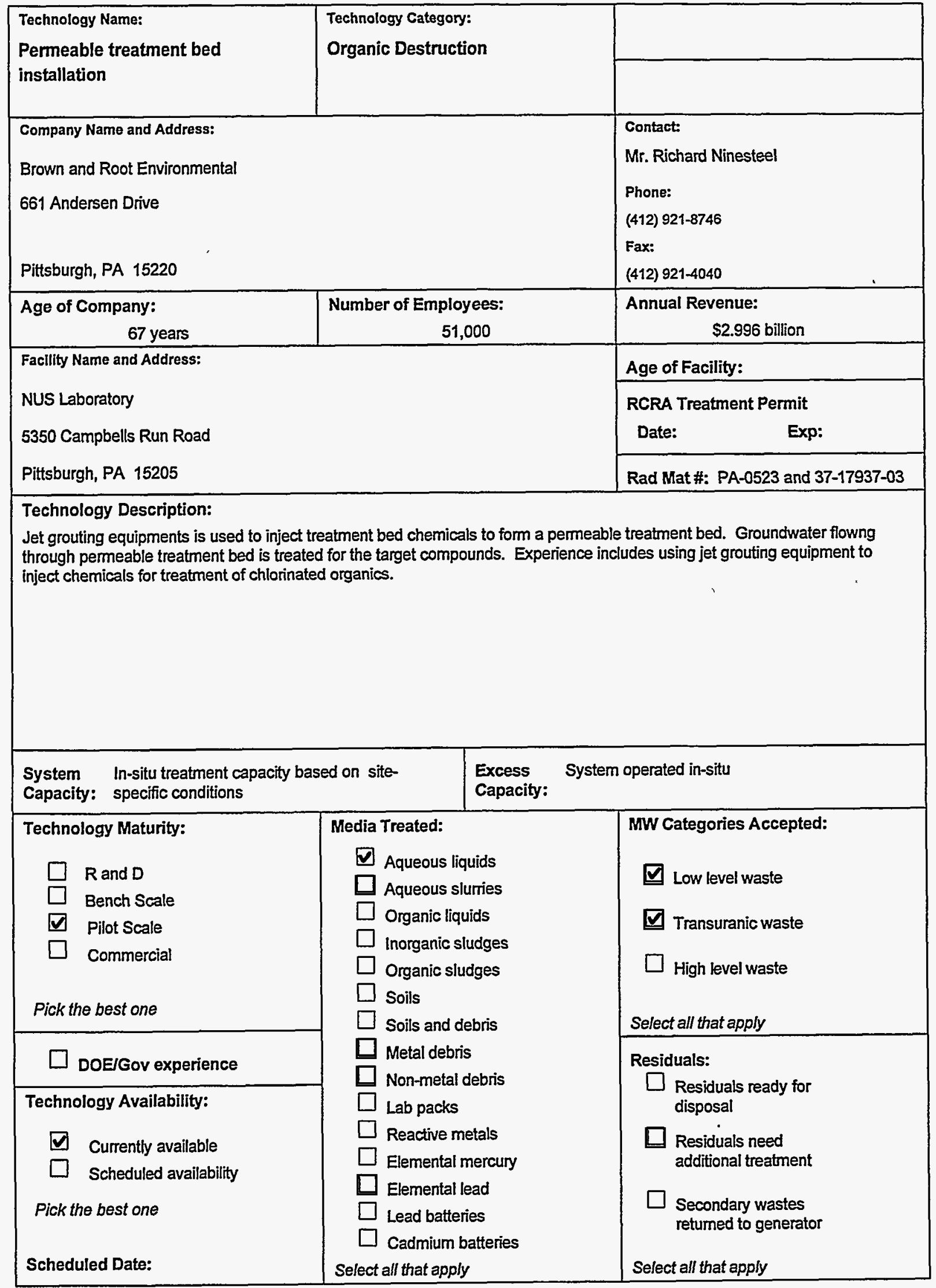


MIXED WASTE TREATABILITY STUDY TECHNOLOGY QUESTIONAIRE

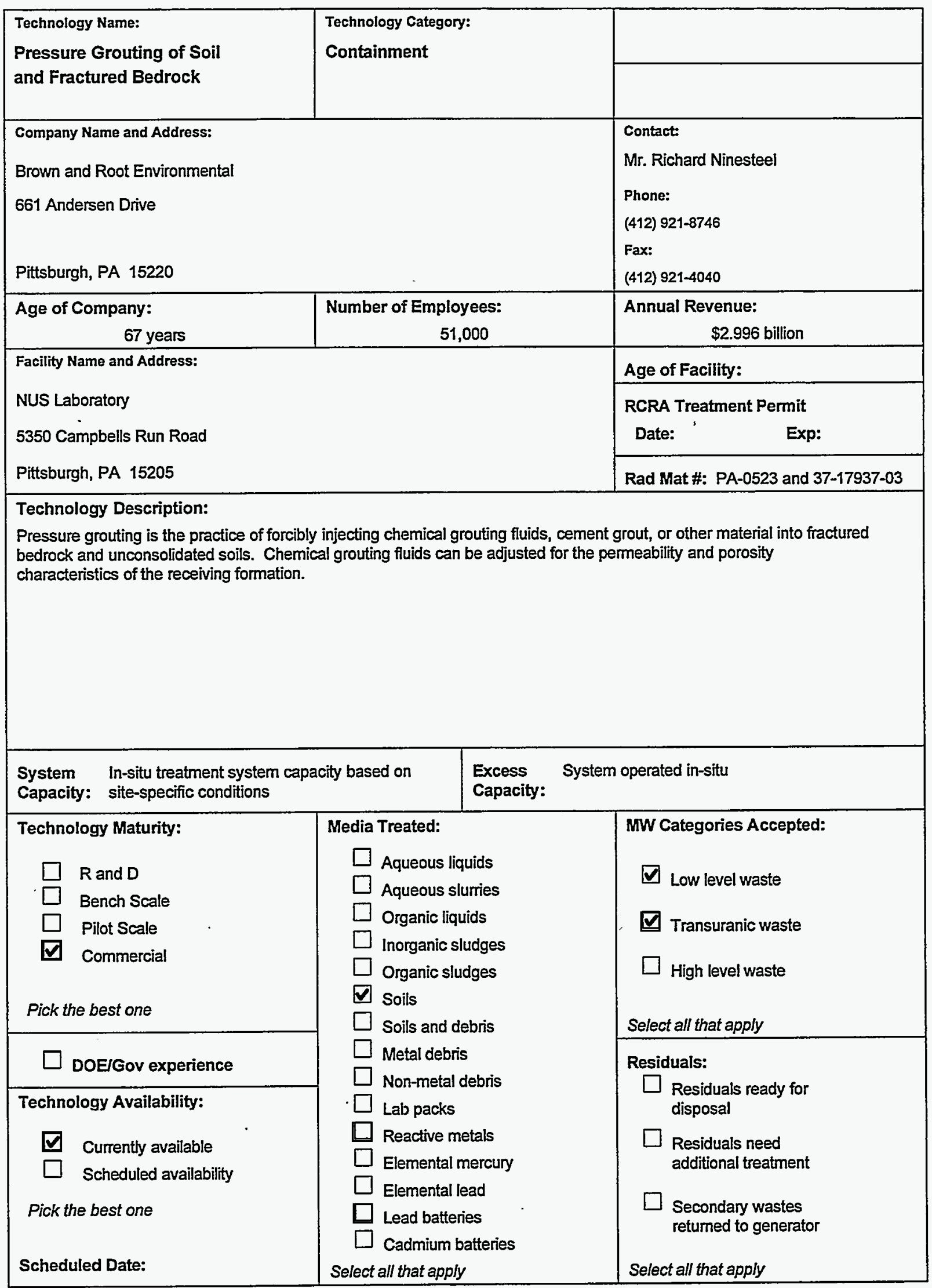




\section{MIXED WASTE TREATABILITY STUDY TECHNOLOGY QUESTIONAIRE}

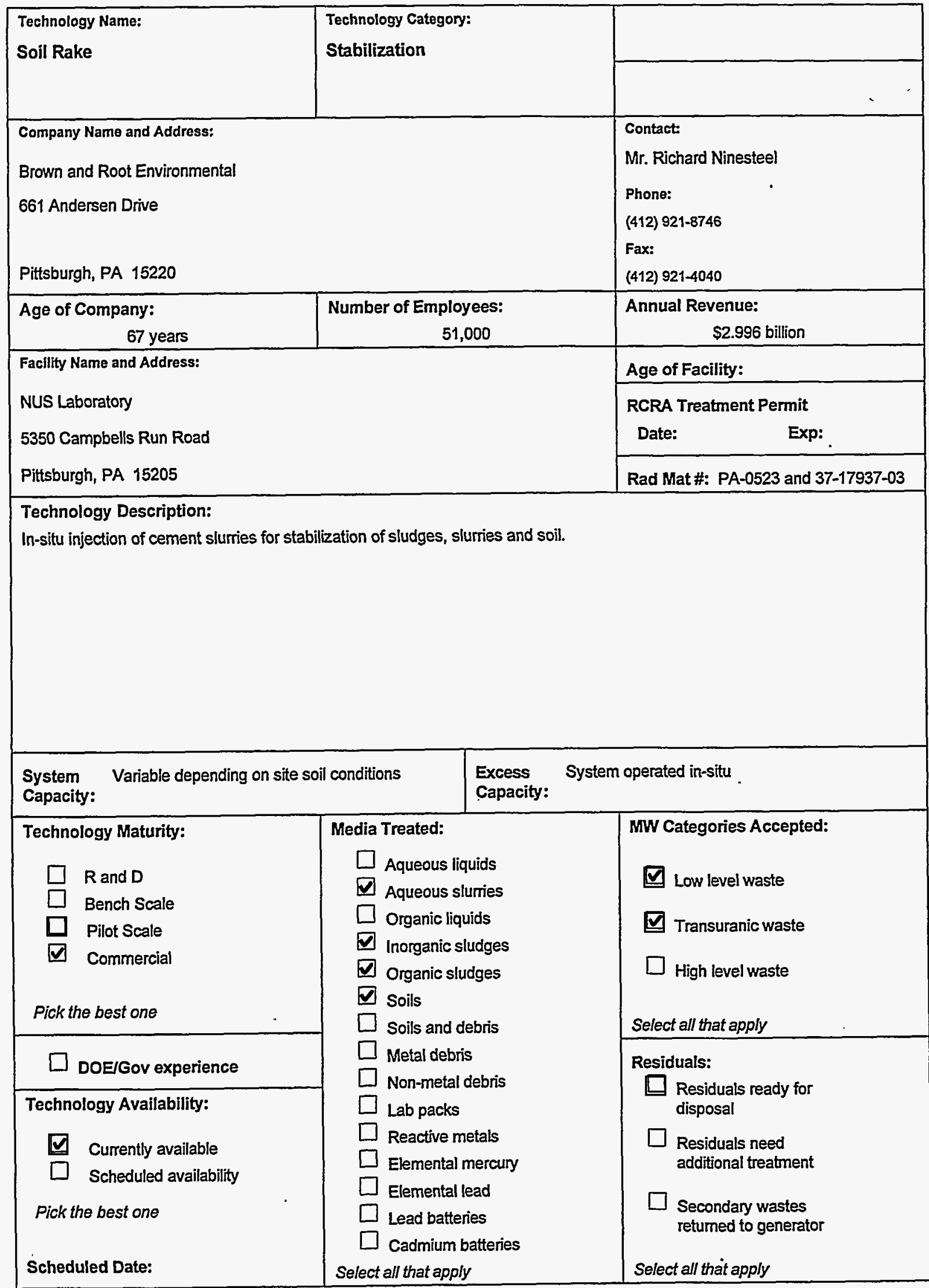


MIXED WASTE TREATABILITY STUDY TECHNOLOGY QUESTIONAIRE

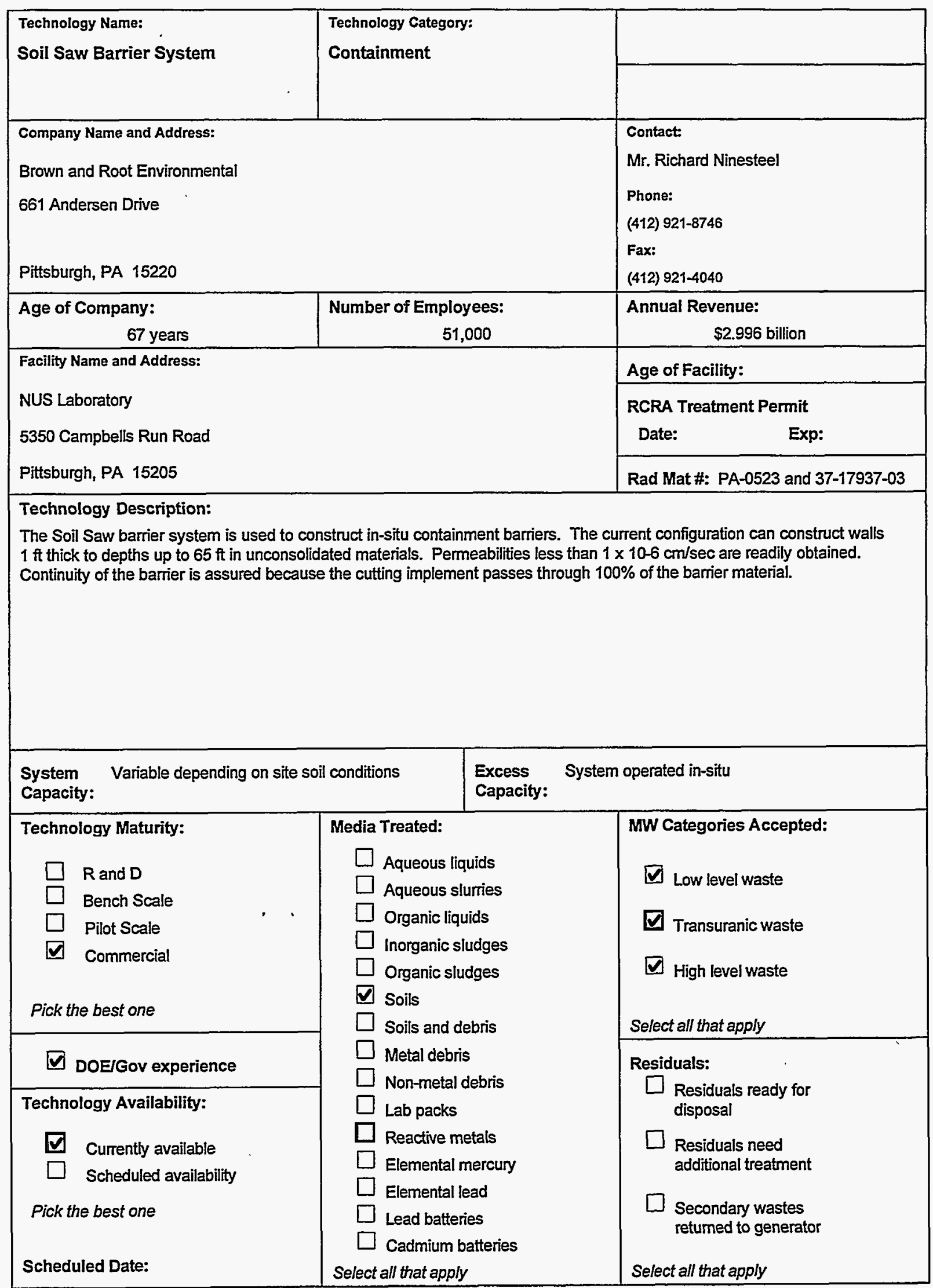


MIXED WASTE TREATABILITY STUDY TECHNOLOGY QUESTIONAIRE

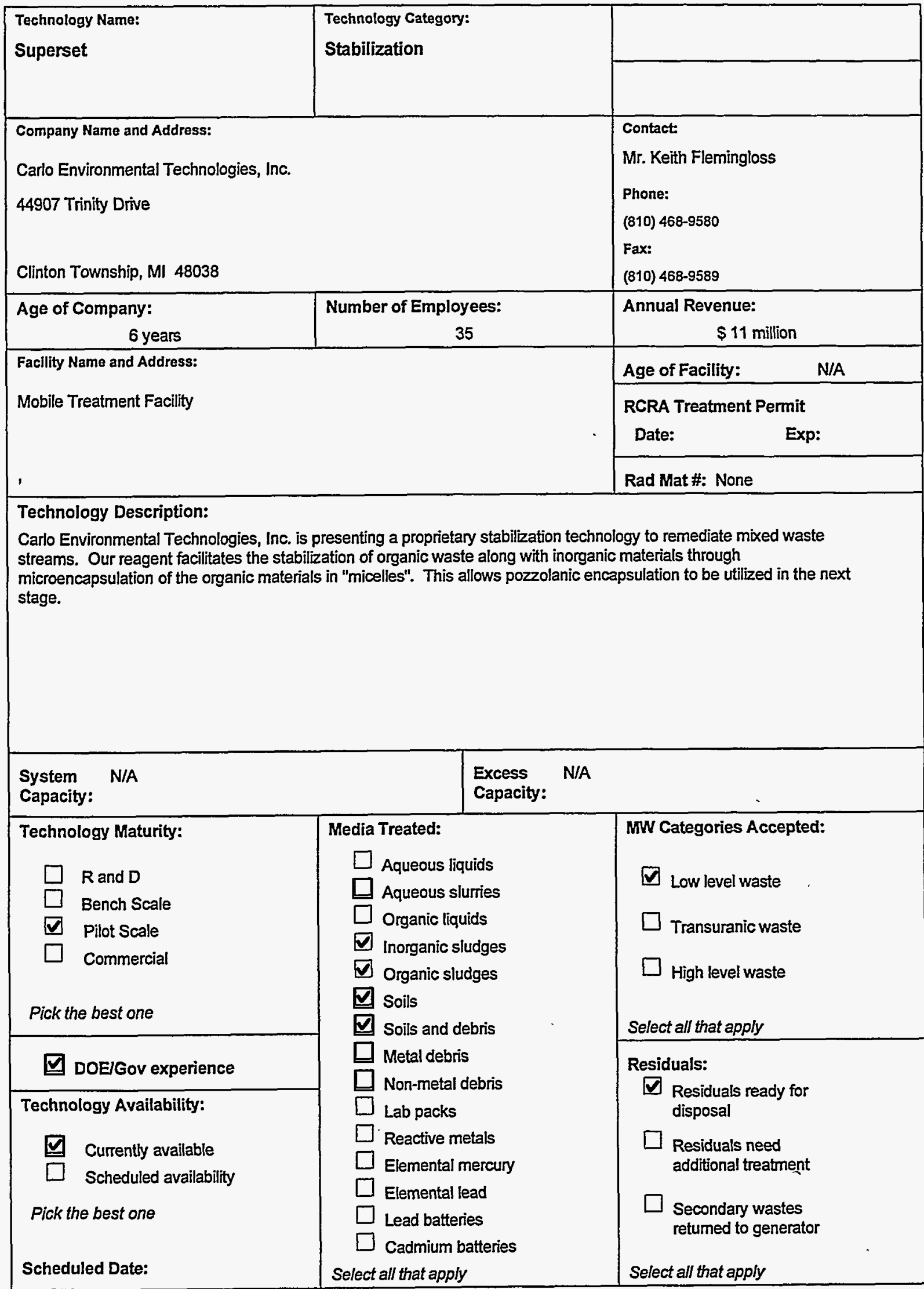




\section{MIXED WASTE TREATABILITY STUDY TECHNOLOGY QUESTIONAIRE}

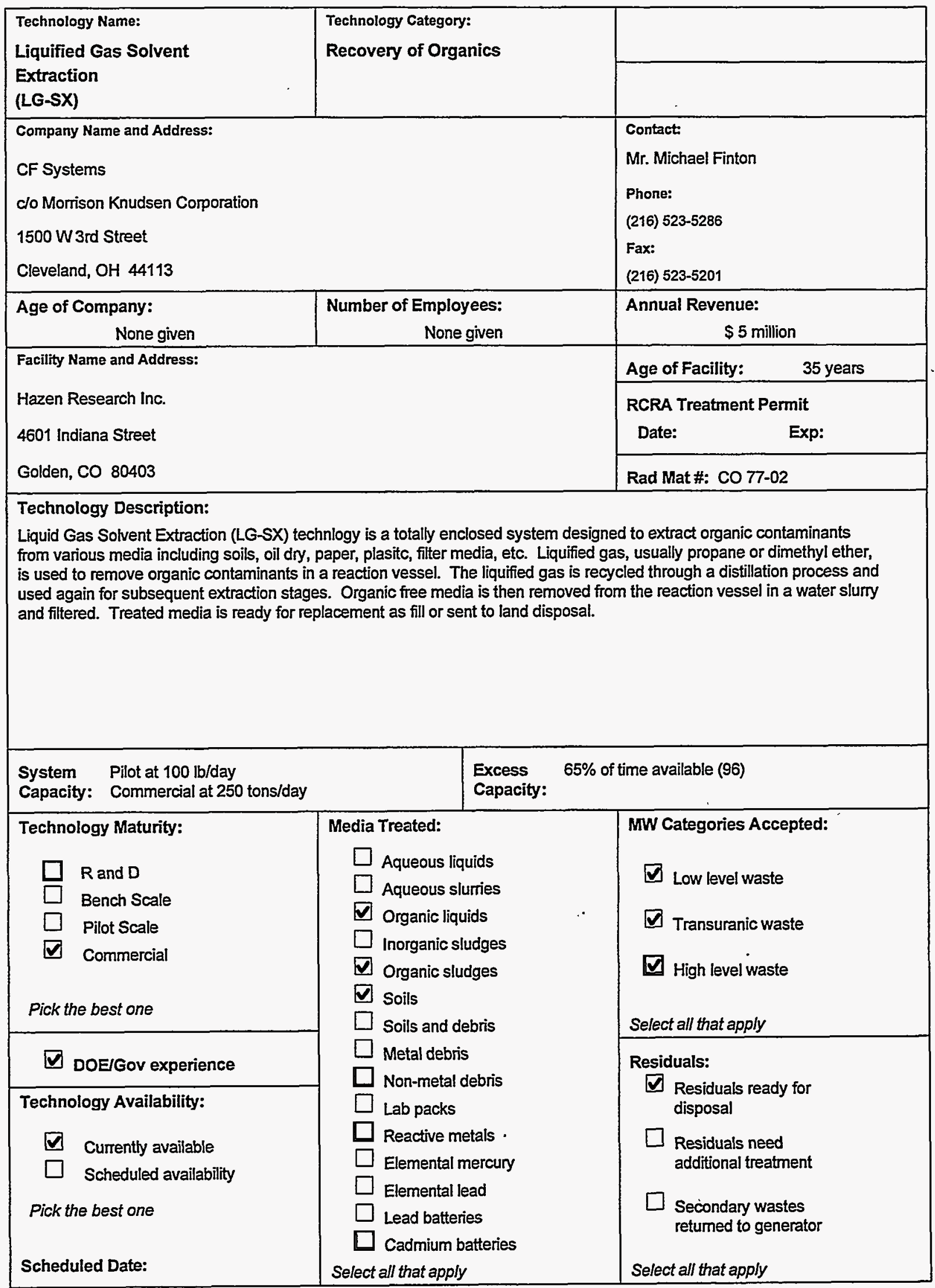


MIXED WASTE TREATABILITY STUDY TECHNOLOGY QUESTIONAIRE

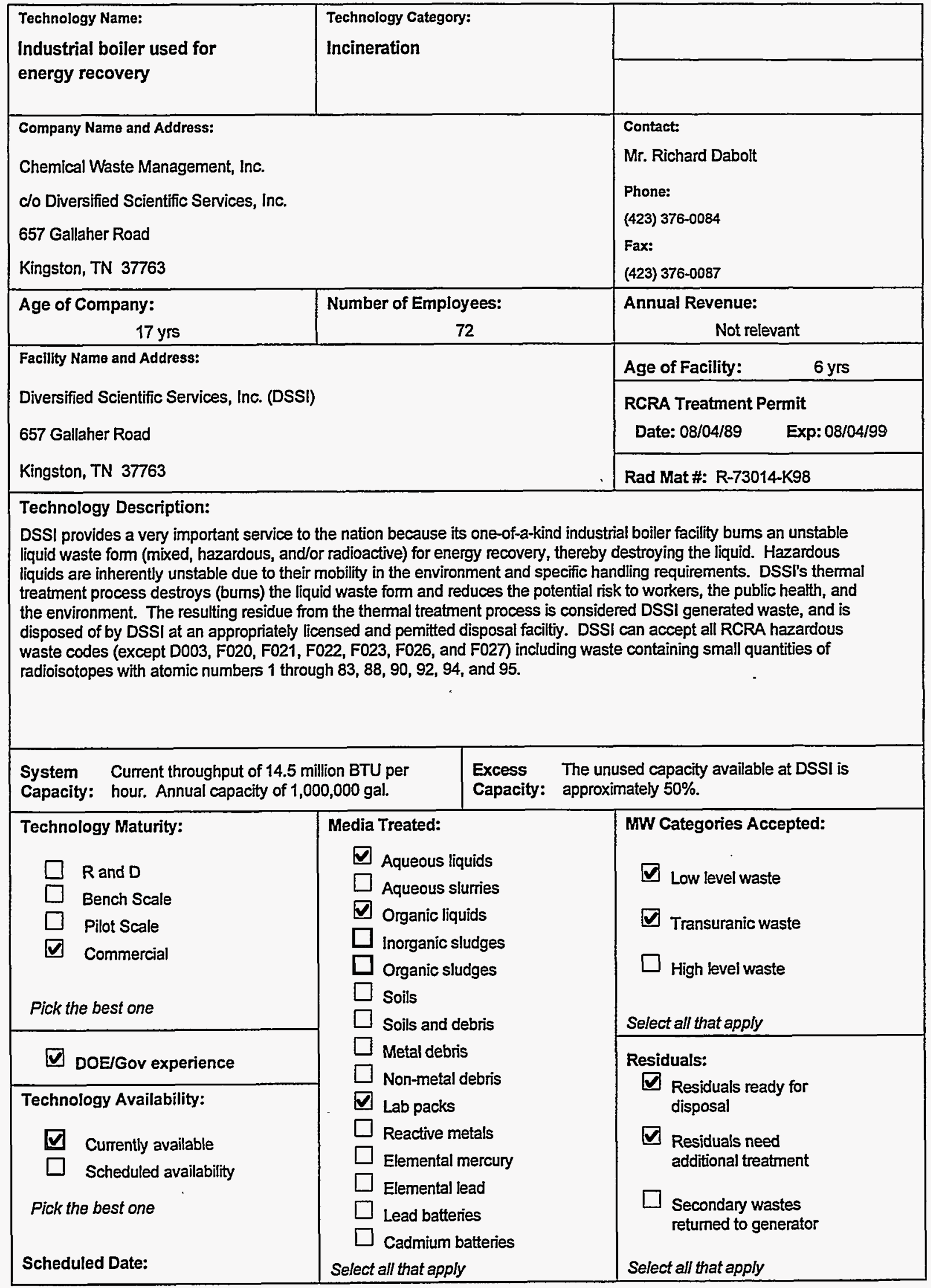


MIXED WASTE TREATABILITY STUDY TECHNOLOGY QUESTIONAIRE

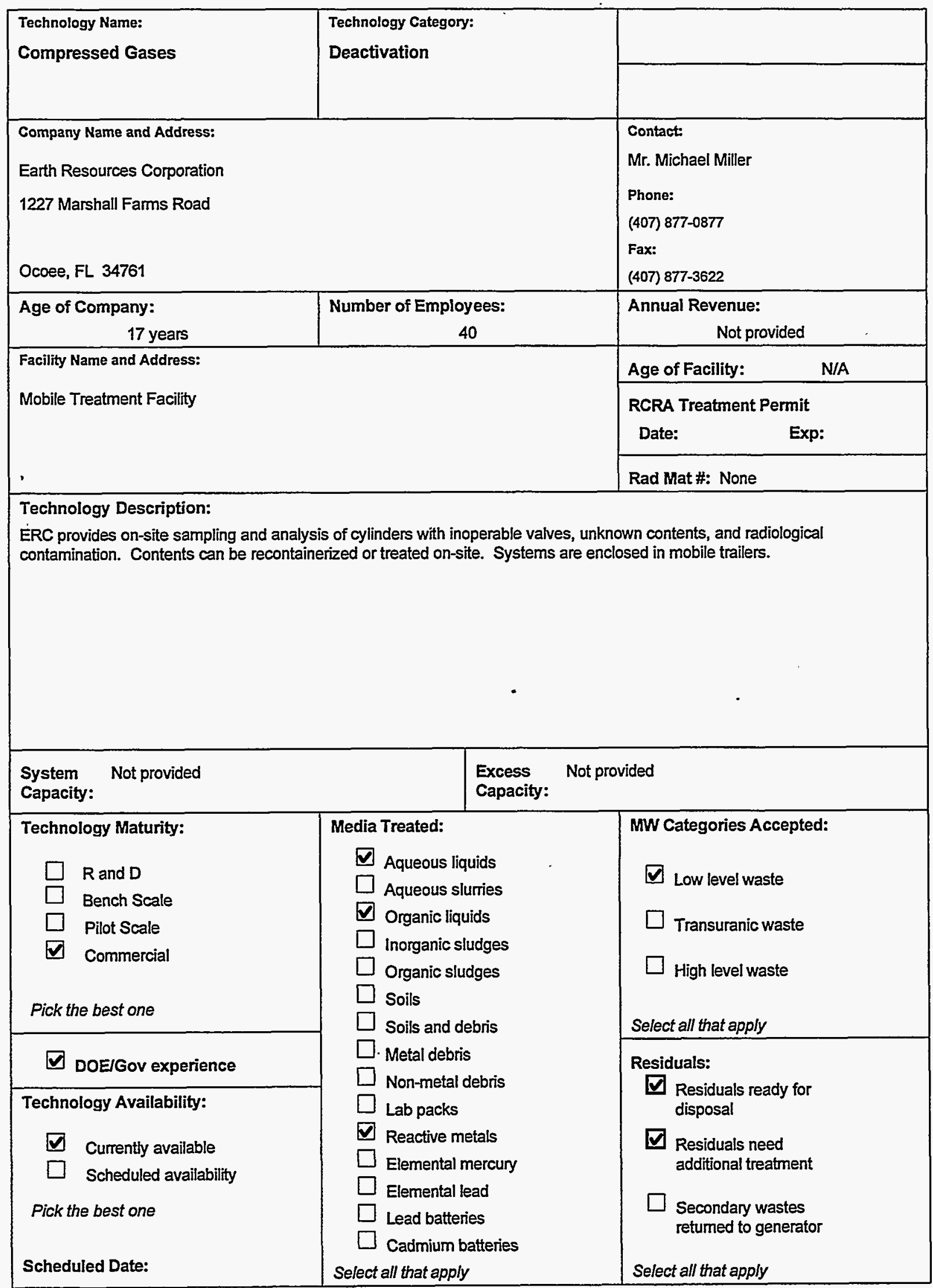


MIXED WASTE TREATABILITY STUDY TECHNOLOGY QUESTIONAIRE

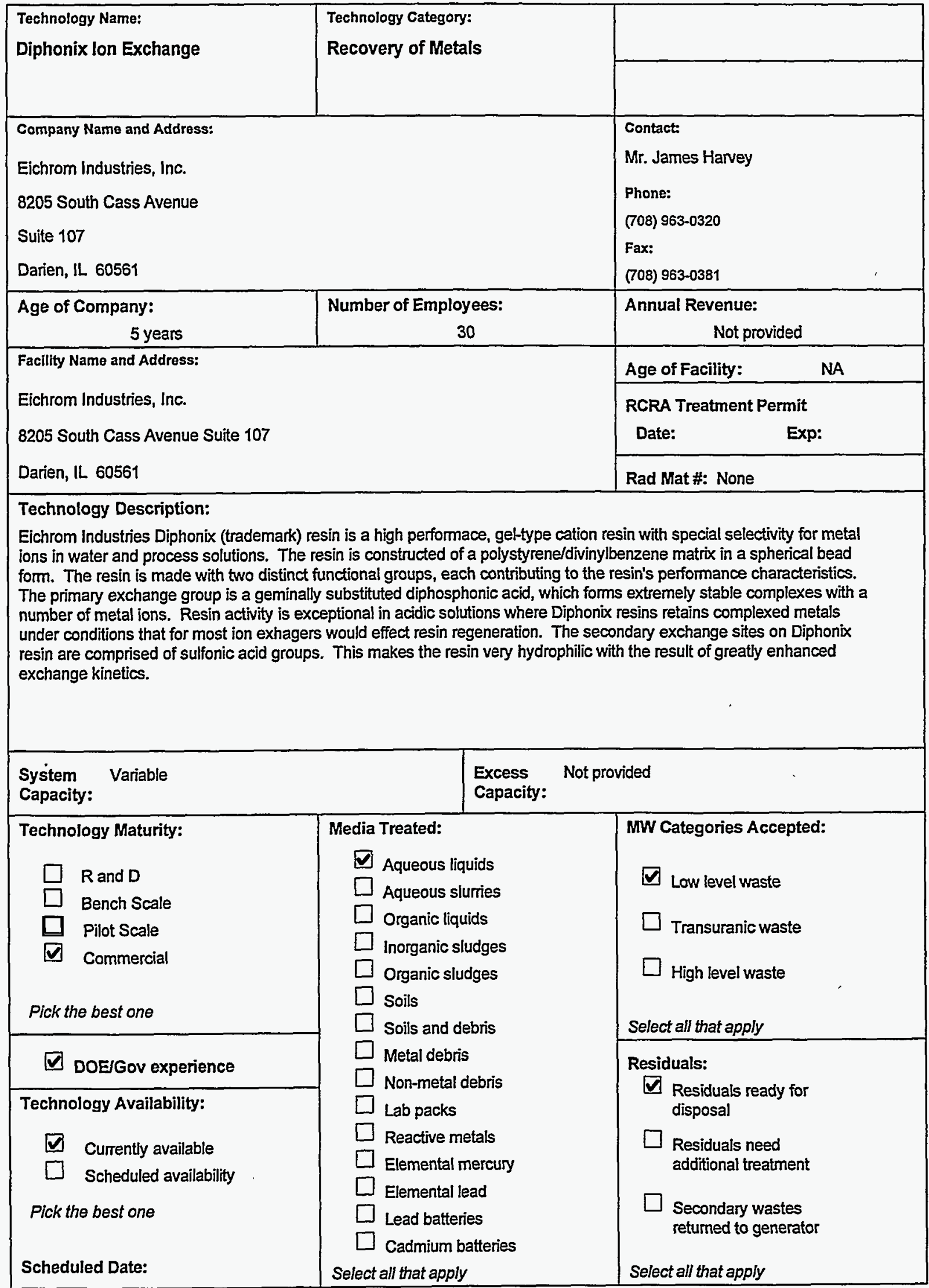


MIXED WASTE TREATABILITY STUDY TECHNOLOGY QUESTIONAIRE

\begin{tabular}{|c|c|c|}
\hline $\begin{array}{l}\text { Technology Name: } \\
\text { DC Graphite Arc Melter }\end{array}$ & $\begin{array}{l}\text { Techriology Category: } \\
\text { Vitrification }\end{array}$ & \\
\hline \multicolumn{2}{|l|}{$\begin{array}{l}\text { Company Name and Address: } \\
\text { Electro-Pyrolysis, Inc. } \\
996 \text { Old Eagle School Road } \\
\text { Wayne, PA } 19087\end{array}$} & $\begin{array}{l}\text { Contact: } \\
\text { Dr. J. Kenneth Wittle } \\
\text { Phone: } \\
(610) 687-9070 \\
\text { Fax: } \\
(610) 964-8570\end{array}$ \\
\hline $\begin{array}{l}\text { Age of Company: } \\
\qquad 13\end{array}$ & $\begin{array}{l}\text { Number of Employees: } \\
\qquad 30 \\
\end{array}$ & $\begin{array}{r}\text { Annual Revenue: } \\
\text { N/A }\end{array}$ \\
\hline \multirow{3}{*}{\multicolumn{2}{|c|}{$\begin{array}{l}\text { Facility Name and Address: } \\
\text { Technology Development Laboratory } \\
304 \text { Directors Dr. } \\
\text { Knoxville, TN } 37923\end{array}$}} & Age of Facility: $\quad 20$ years \\
\hline & & $\begin{array}{l}\text { RCRA Treatment Permit } \\
\text { Date: } \quad \text { Exp: }\end{array}$ \\
\hline & & Rad Mat \#: TN R-01060-J95 \\
\hline \multicolumn{3}{|l|}{$\begin{array}{l}\text { Technology Description: } \\
\text { A DC Graphite Arc Fumace } \\
\text { material and thermally decor } \\
\text { would be protective clothing, } \\
\text { produce similar low level was }\end{array}$} \\
\hline $\begin{array}{l}\text { System } \quad 50 \mathrm{~kg} / \mathrm{hr} \text {. } \\
\text { Capacity: }\end{array}$ & $\begin{array}{l}\text { Excess } \\
\text { Capacity: }\end{array}$ & Variable depending on workload from 0 to $100 \%$ \\
\hline $\begin{array}{l}\text { Technology Maturity: } \\
\square \text { R and D } \\
\square \text { Bench Scale } \\
\square \text { Pilot Scale } \\
\square \text { Commercial } \\
\text { Pick the best one }\end{array}$ & \multirow{3}{*}{$\begin{array}{l}\text { Media Treated: } \\
\square \text { Aqueous liquids } \\
\square \text { Aqueous slurries } \\
\square \text { Organic liquids } \\
\square \text { Inorganic sludges } \\
\square \text { Organic sludges } \\
\square \text { Soils } \\
\square \text { Soils and debris } \\
\square \text { Metal debris } \\
\square \text { Non-metal debris } \\
\square \text { Lab packs } \\
\square \text { Reactive metals } \\
\square \text { Elemental mercury } \\
\square \text { Elemental lead. } \\
\square \text { Lead batteries } \\
\square \text { Cadmium batteries } \\
\text { Select all that apply }\end{array}$} & $\begin{array}{l}\text { MW Categories Accepted: } \\
\square \text { Low level waste } \\
\square \text { Transuranic waste } \\
\square \text { High level waste } \\
\text { Select all that apply }\end{array}$ \\
\hline$\square$ DOE/Gov experience & & Residuals: \\
\hline $\begin{array}{l}\text { Technology Availability: } \\
\square \text { Currently available } \\
\square \text { Scheduled availability } \\
\text { Pick the best one } \\
\text { Scheduled Date: }\end{array}$ & & $\begin{array}{l}\square \text { Residuals need } \\
\text { additional treatment } \\
\text { disposal } \\
\text { Secondary wastes } \\
\text { returned to generator }\end{array}$ \\
\hline
\end{tabular}




\section{MIXED WASTE TREATABILITY STUDY TECHNOLOGY QUESTIONAIRE}

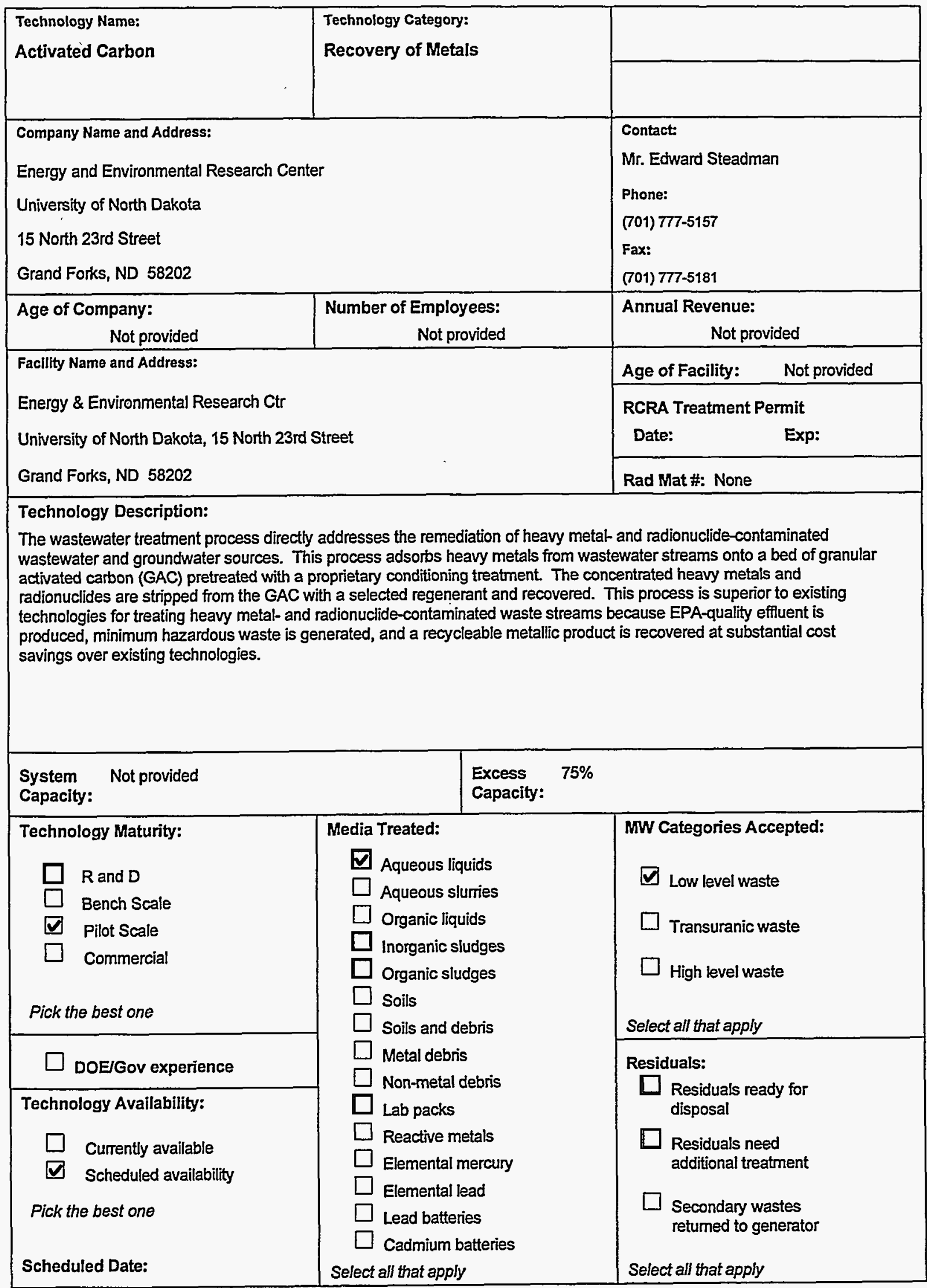


MIXED WASTE TREATABILITY STUDY TECHNOLOGY QUESTIONAIRE

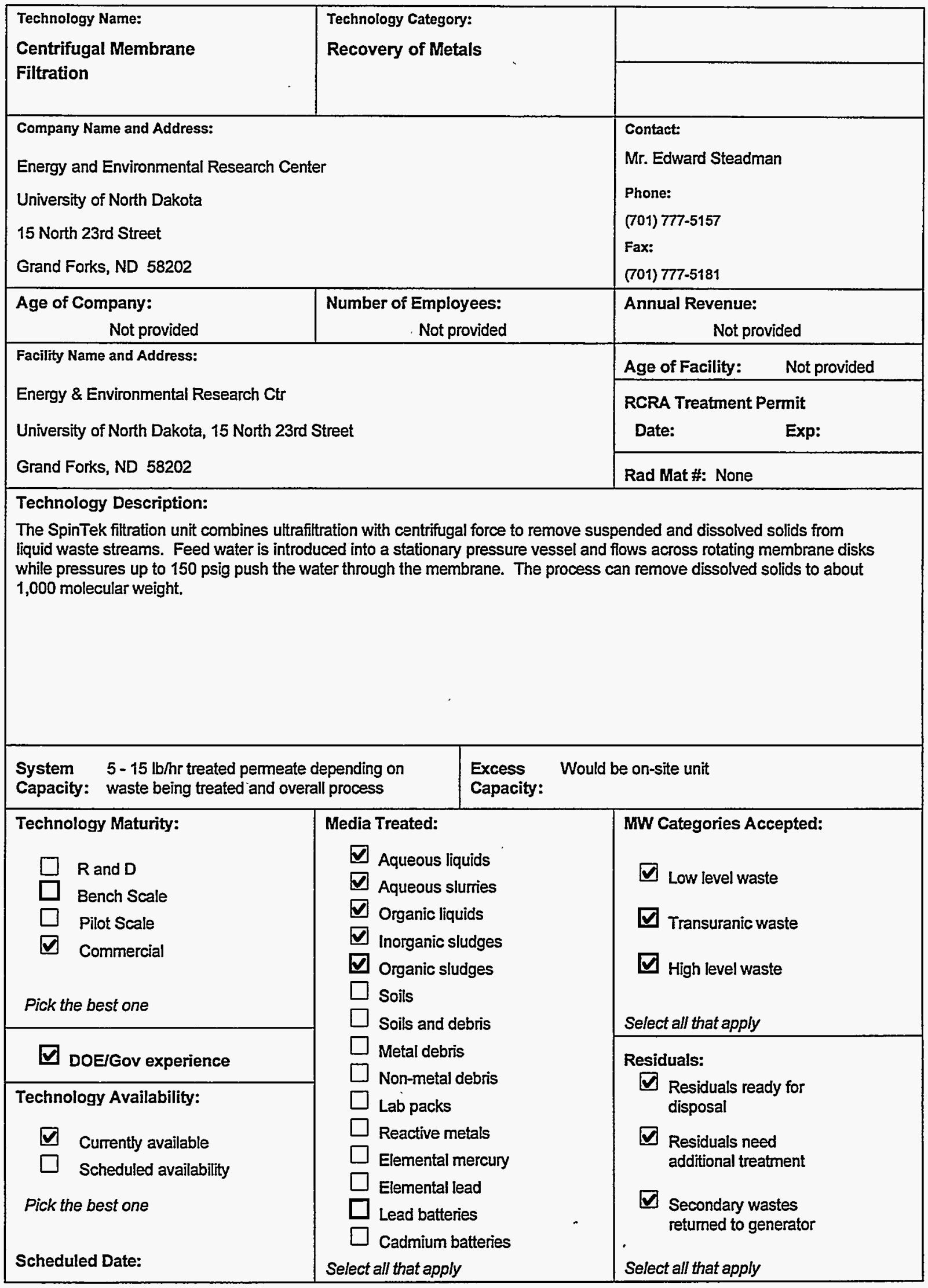


MIXED WASTE TREATABILITY STUDY TECHNOLOGY QUESTIONAIRE

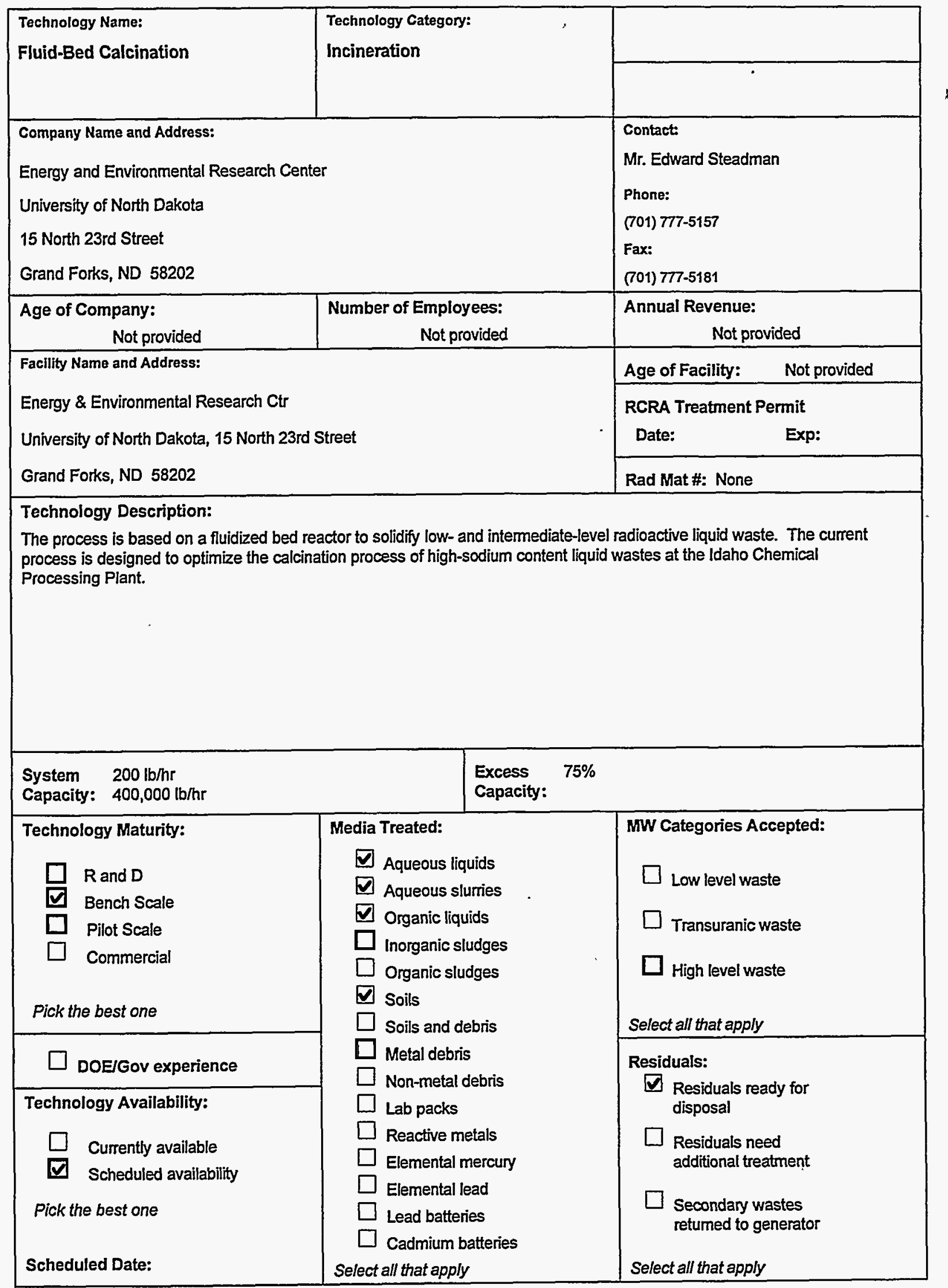


MIXED WASTE TREATABILITY STUDY TECHNOLOGY QUESTIONAIRE

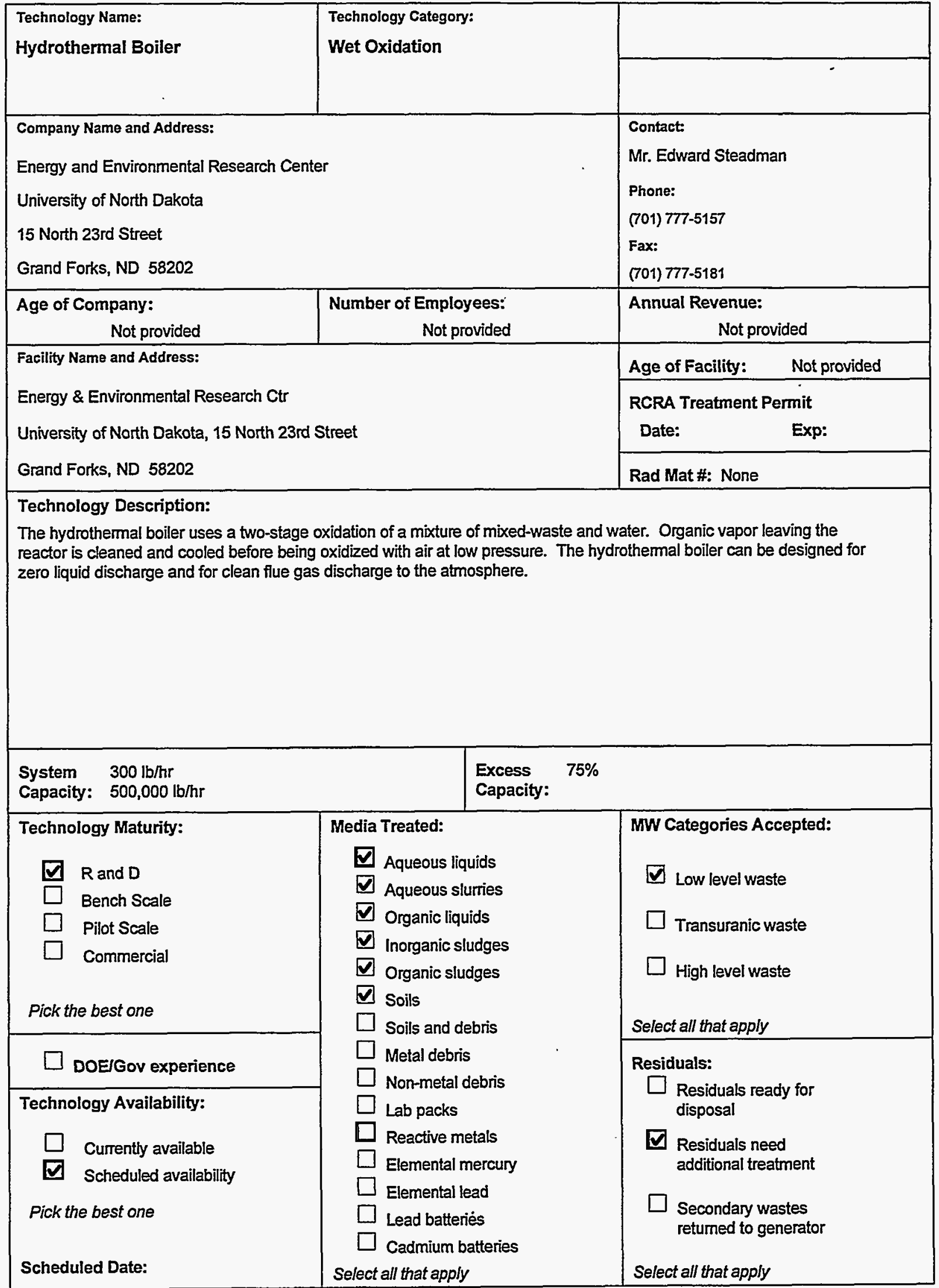


MIXED WASTE TREATABILITY STUDY TECHNOLOGY QUESTIONAIRE

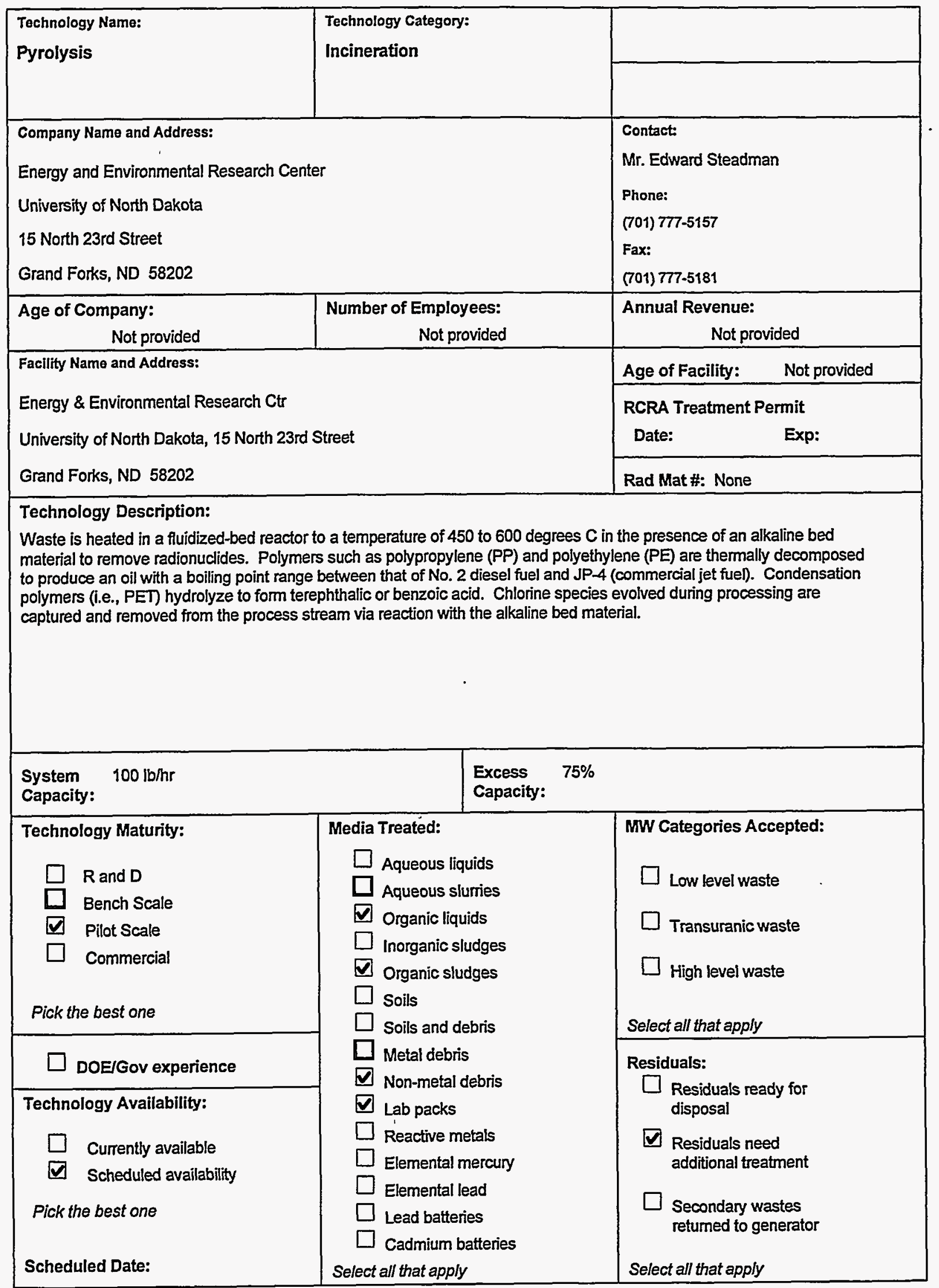


MIXED WASTE TREATABILITY STUDY TECHNOLOGY QUESTIONAIRE

\begin{tabular}{|c|c|c|}
\hline $\begin{array}{l}\text { Technology Name: } \\
\text { Macroencapsulation }\end{array}$ & $\begin{array}{l}\text { Technology Category: } \\
\text { Macroencapsulation }\end{array}$ & \\
\hline \multicolumn{2}{|l|}{$\begin{array}{l}\text { Company Name and Address: } \\
\text { Envirocare of Utah, Inc. } \\
46 \text { West Broadway } \\
\text { Suite } 240 \\
\text { Salt Lake City, UT } 84101\end{array}$} & $\begin{array}{l}\text { Contact: } \\
\text { Mr. Kevin Fuller } \\
\text { Phone: } \\
\text { (801) 532-1330 } \\
\text { Fax: } \\
\text { (801) } 537-7345\end{array}$ \\
\hline $\begin{array}{r}\text { Age of Company: } \\
8 \text { years } \\
\end{array}$ & $\begin{array}{c}\text { Number of Employees: } \\
70 \\
\end{array}$ & $\begin{array}{r}\text { Annual Revenue: } \\
\text { N/A }\end{array}$ \\
\hline \multirow{3}{*}{\multicolumn{2}{|c|}{$\begin{array}{l}\text { Facility Name and Address: } \\
\text { Envirocare of Utah, Inc. } \\
\text { US 1-80, Exit } 49 \text { Tooele County } \\
\text { Clive, UT } 84029\end{array}$}} & Age of Facility: $\quad$ 1year \\
\hline & & $\begin{array}{l}\text { RCRA Treatment Permit } \\
\begin{array}{ll}\text { Date: } 01 / 01 / 90 & \text { Exp: } 11 / 30 / 00\end{array}\end{array}$ \\
\hline & & Rad Mat \#: UT 2300240 \\
\hline \multicolumn{3}{|l|}{$\begin{array}{l}\text { Technology Description: } \\
\text { Elemental lead pieces/articles a } \\
\text { surround the waste by a minimb }\end{array}$} \\
\hline $\begin{array}{l}\text { System } 150 \text { tons/day } \\
\text { Capacity: }\end{array}$ & $\begin{array}{l}\text { Excess } \\
\text { Capacity }\end{array}$ & Ivailability \\
\hline $\begin{array}{c}\text { Technology Maturity: } \\
\square \quad \text { R and D } \\
\square \text { Bench Scale } \\
\square \quad \text { Pilot Scale } \\
\square \quad \text { Commercial } \\
\text { Pick the best one }\end{array}$ & \multirow{3}{*}{$\begin{array}{l}\text { Media Treated: } \\
\square \text { Aqueous liquids } \\
\square \text { Aqueous slurries } \\
\square \text { Organic liquids } \\
\square \text { Inorganic sludges } \\
\square \text { Organic sludges } \\
\square \text { Soils } \\
\square \text { Soils and debris } \\
\square \text { Metal debris } \\
\square \text { Non-metal debris } \\
\square \text { Lab packs } \\
\square \text { Reactive metals } \\
\square \text { Elemental mercury } \\
\square \text { Elemental lead } \\
\square \text { Lead batteries } \\
\square \text { Cadmium batteries } \\
\text { Select all that apply }\end{array}$} & $\begin{array}{l}\text { MW Categories Accepted: } \\
\square \text { Low level waste } \\
\square \text { Transuranic waste } \\
\text { High level waste } \\
\text { Select all that apply }\end{array}$ \\
\hline$\checkmark$ DOEIGov experience & & $\begin{array}{l}\text { Residuals: } \\
\square \text { Residuals readv for }\end{array}$ \\
\hline $\begin{array}{l}\text { Technology Availability: } \\
\qquad \begin{array}{l}\square \\
\text { Currentty available } \\
\square \text { Scheduled availability }\end{array} \\
\text { Pick the best one } \\
\text { Scheduled Date: }\end{array}$ & & $\begin{array}{l}\square \text { Residuals need } \\
\text { additional treatment } \\
\square \text { Secondary wastes } \\
\text { returned to generator } \\
\text { Select all that apply }\end{array}$ \\
\hline
\end{tabular}


MIXED WASTE TREATABILITY STUDY TECHNOLOGY QUESTIONAIRE

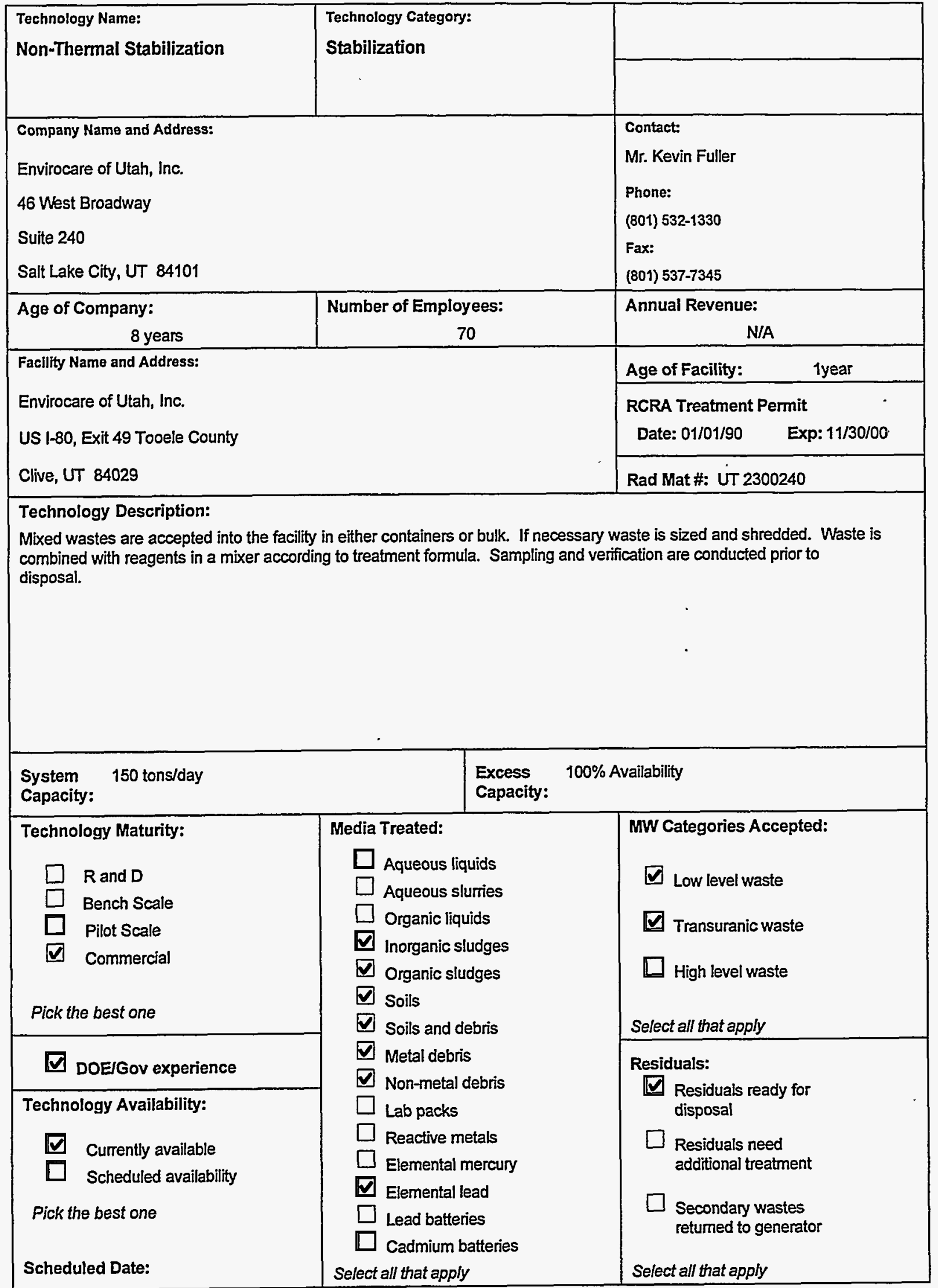




\section{MIXED WASTE TREATABILITY STUDY TECHNOLOGY QUESTIONAIRE}

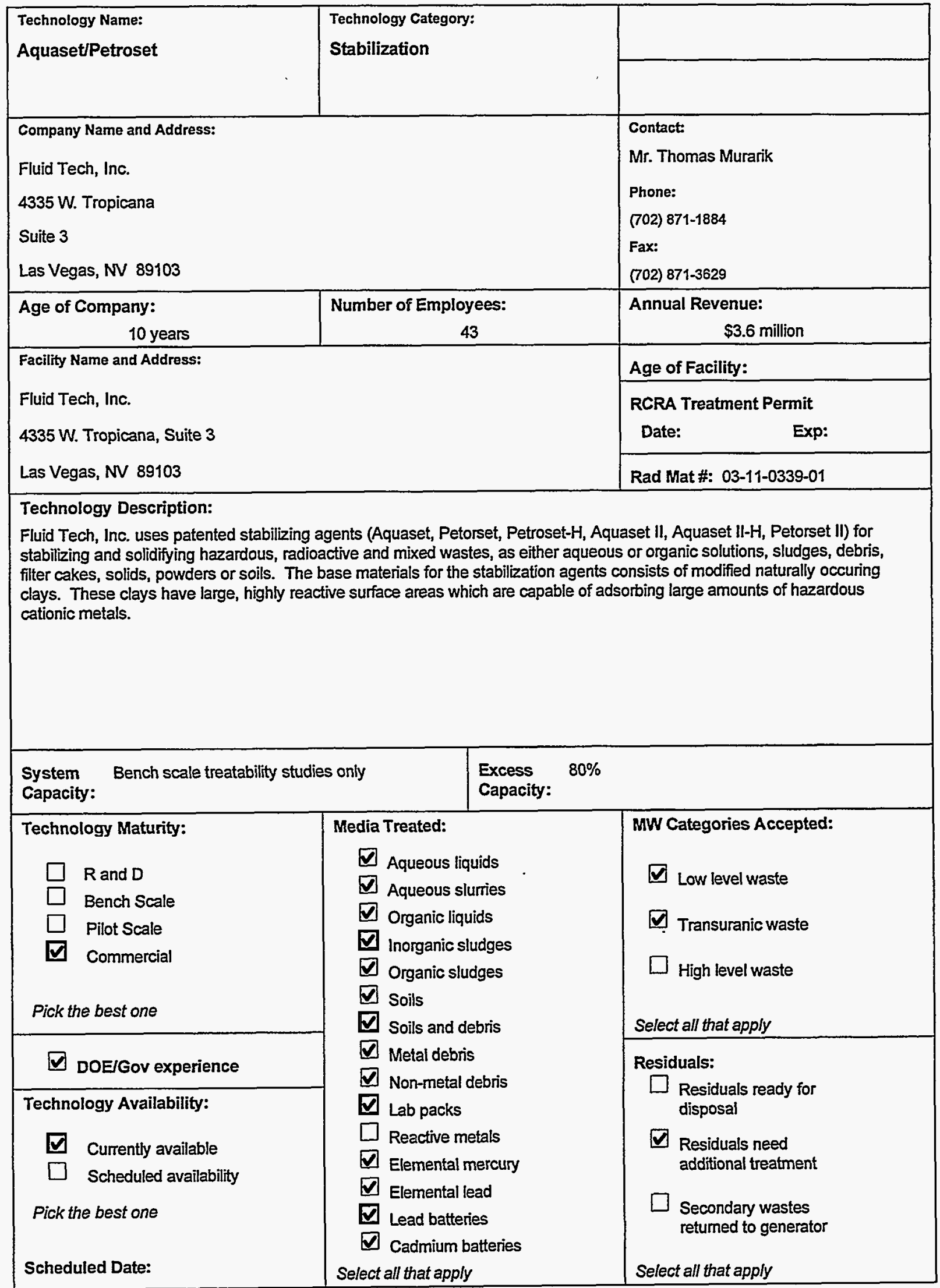


MIXED WASTE TREATABILITY STUDY TECHNOLOGY QUESTIONAIRE

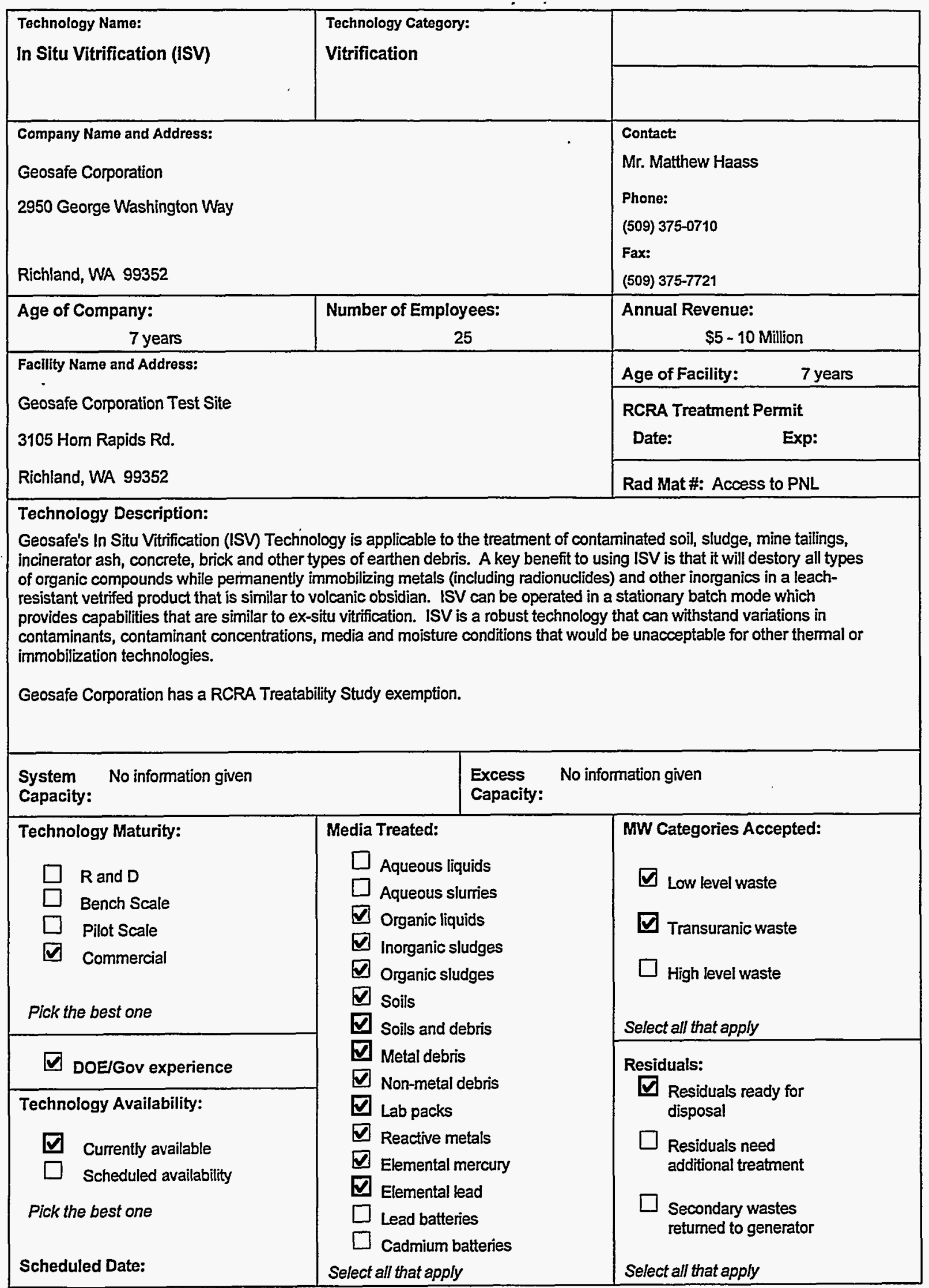


MIXED WASTE TREATABILITY STUDY TECHNOLOGY QUESTIONAIRE

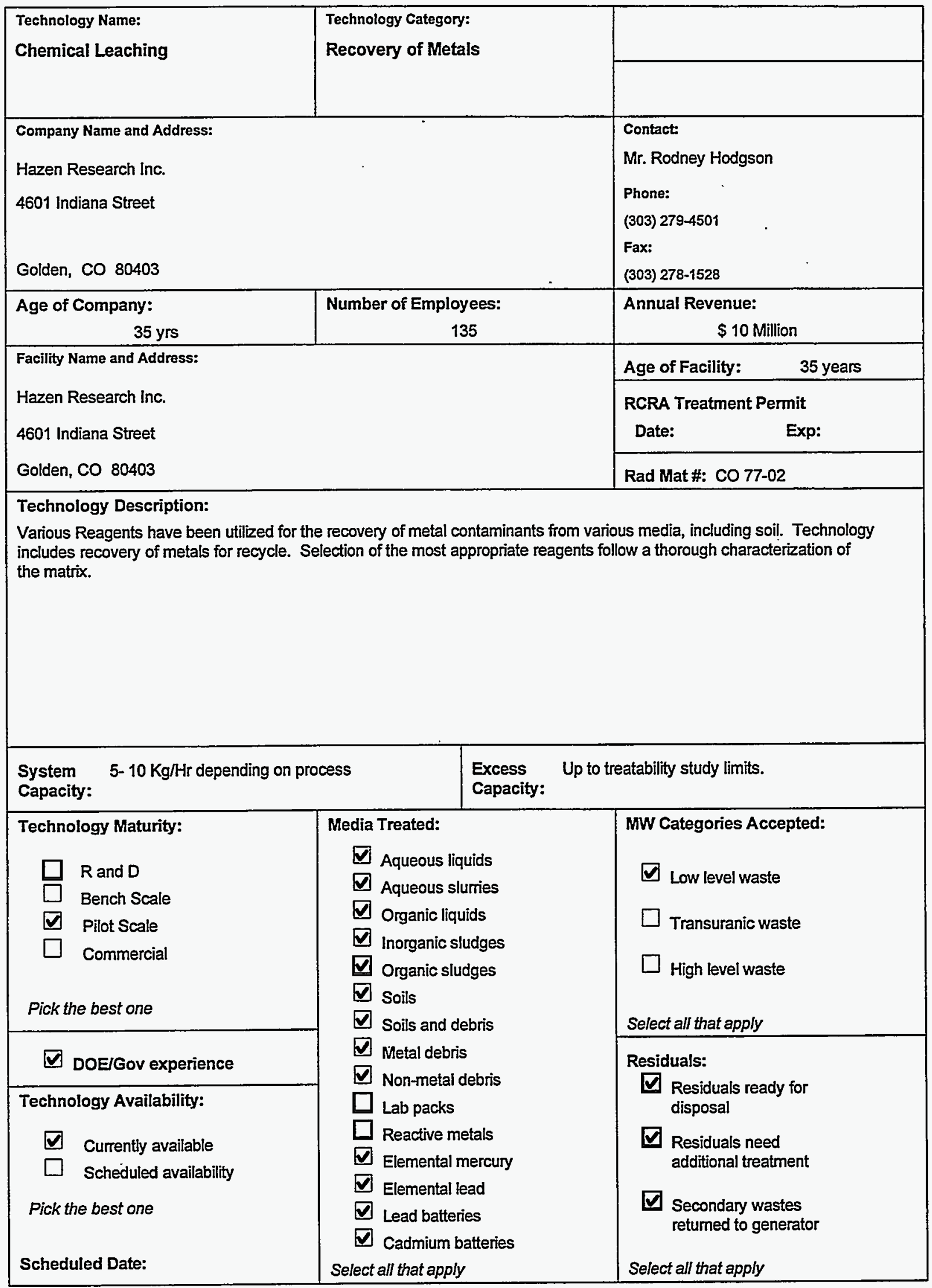


MIXED WASTE TREATABILITY STUDY TECHNOLOGY QUESTIONAIRE

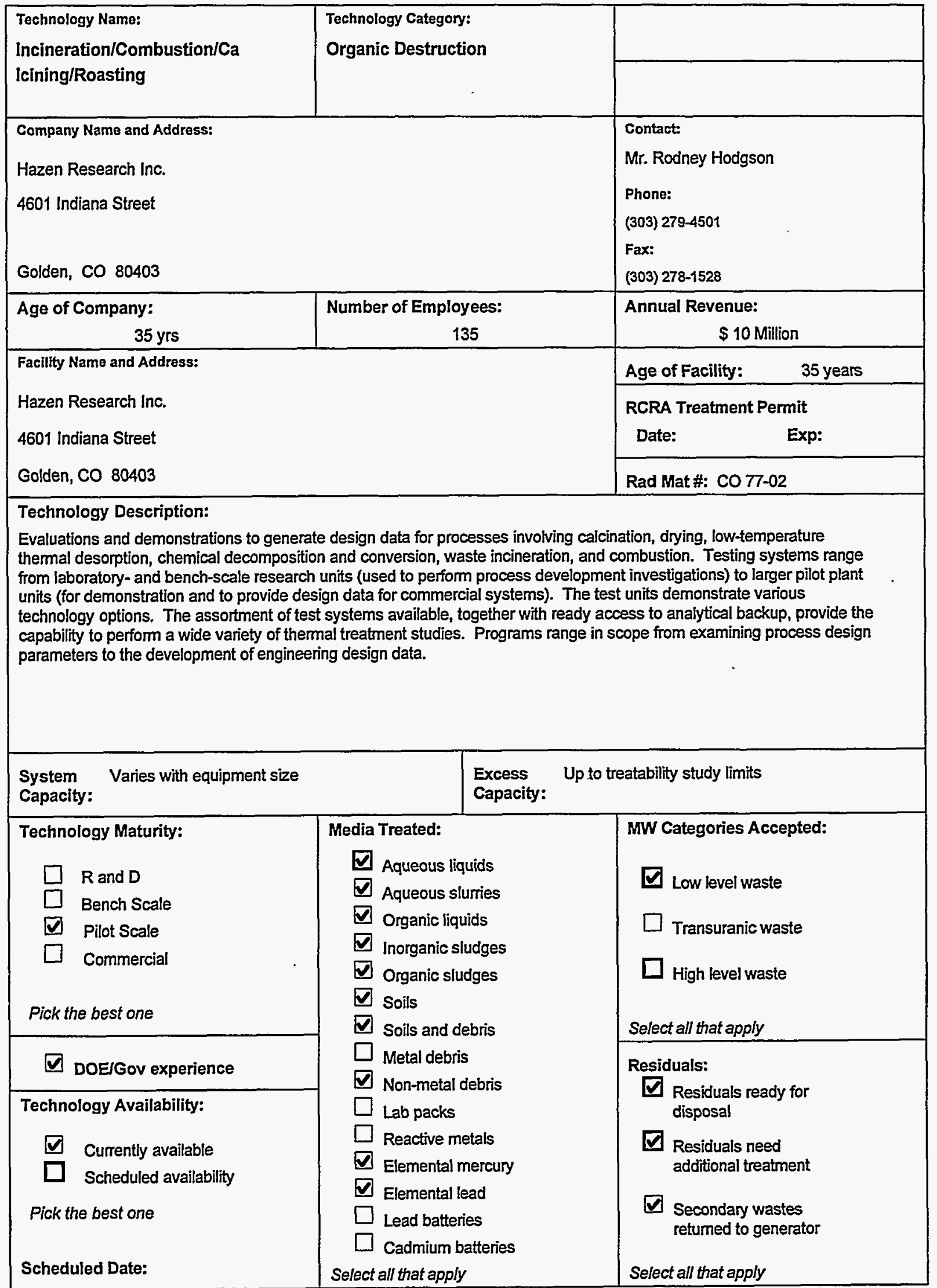


MIXED WASTE TREATABILITY STUDY TECHNOLOGY QUESTIONAIRE

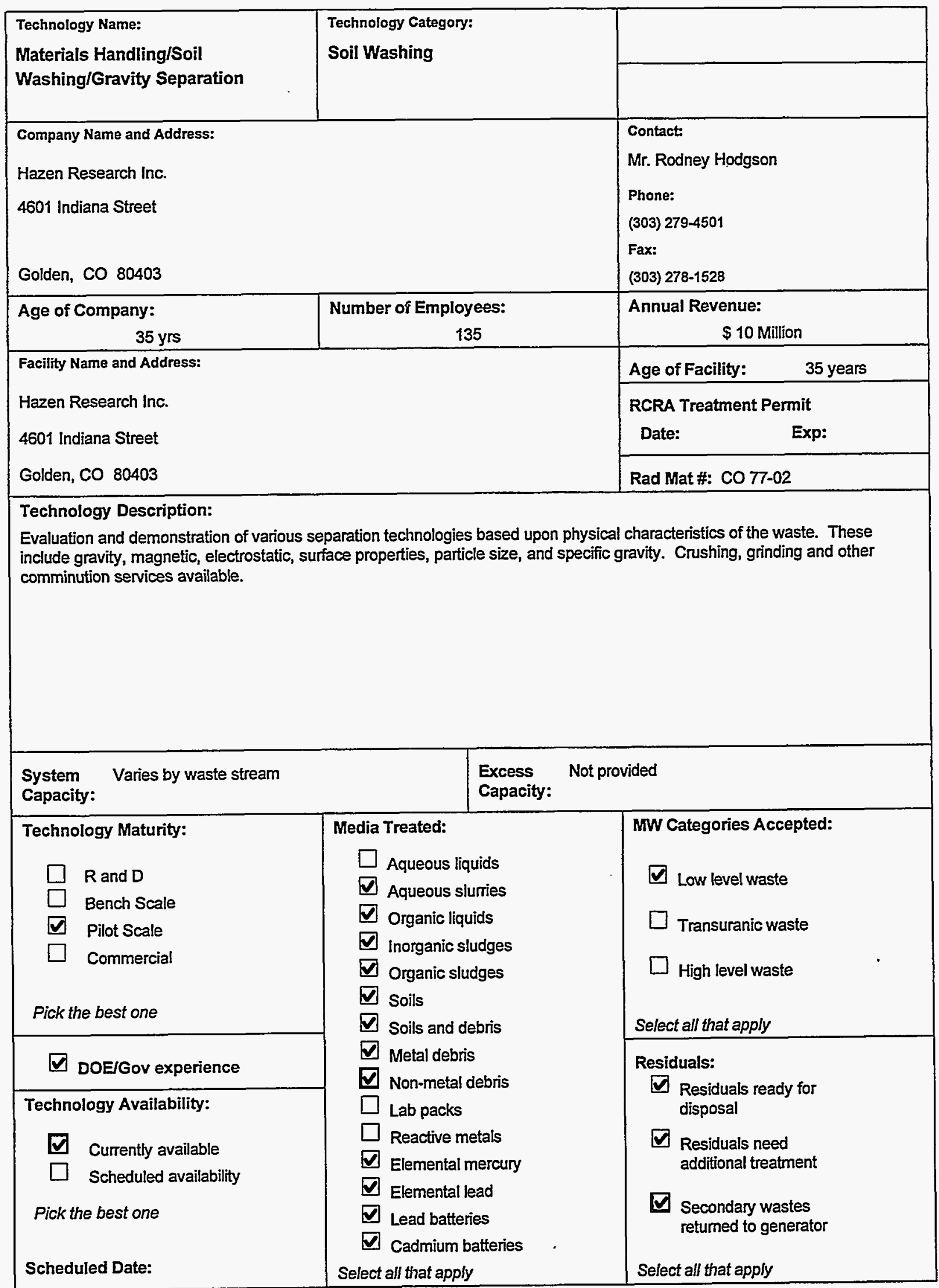


MIXED WASTE TREATABILITY STUDY TECHNOLOGY QUESTIONAIRE

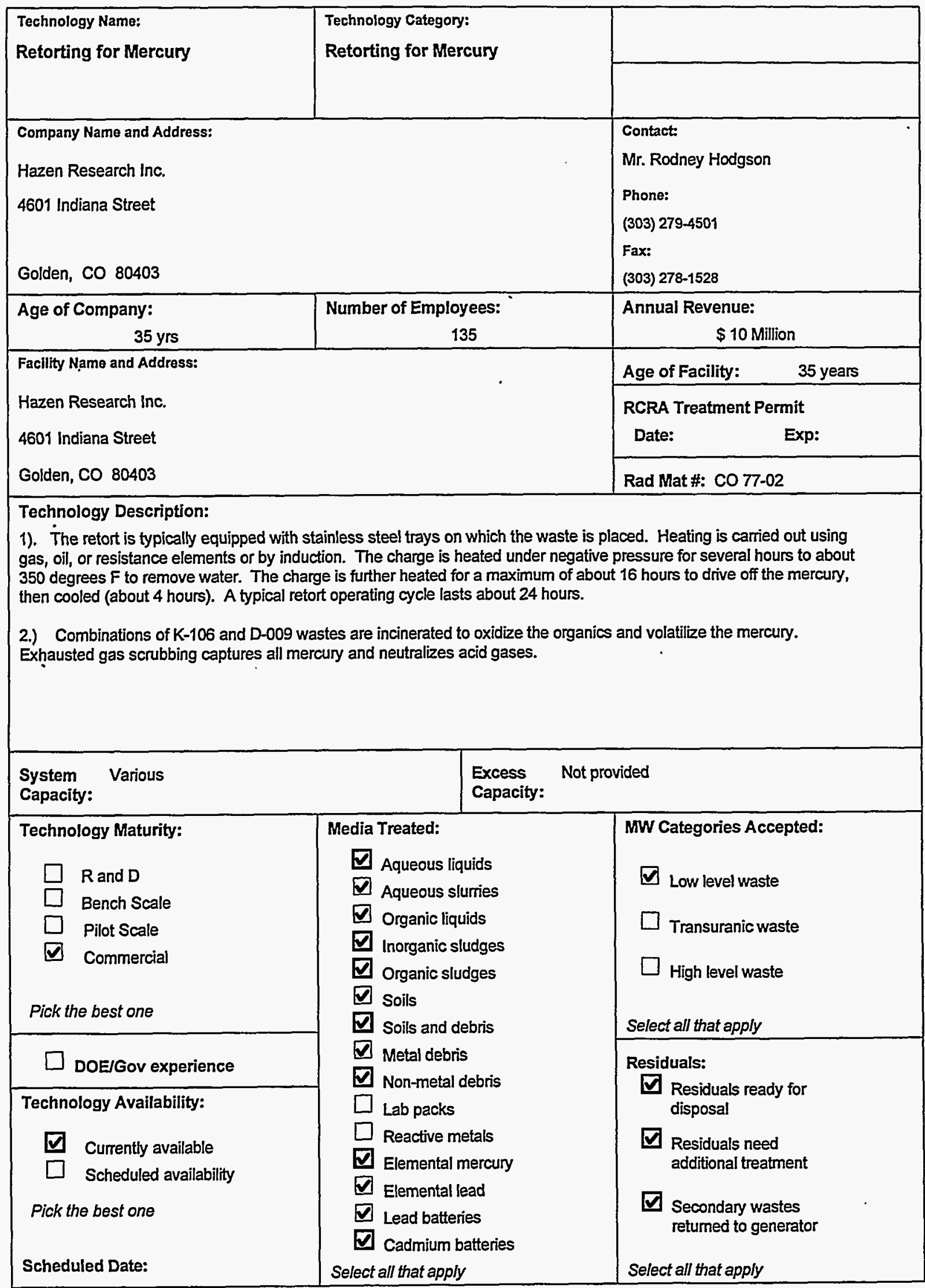


MIXED WASTE TREATABILITY STUDY TECHNOLOGY QUESTIONAIRE

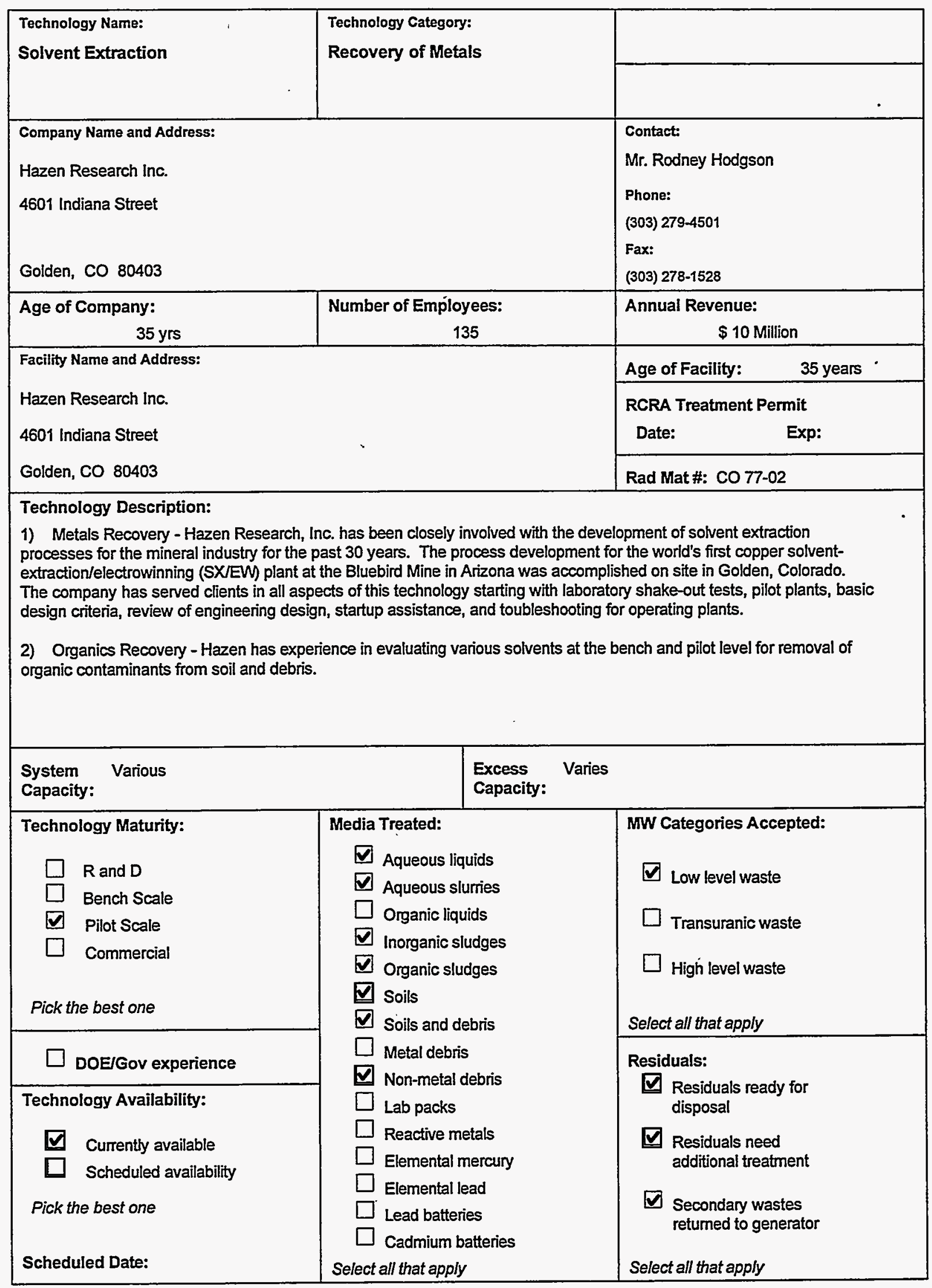


MIXED WASTE TREATABILITY STUDY TECHNOLOGY QUESTIONAIRE

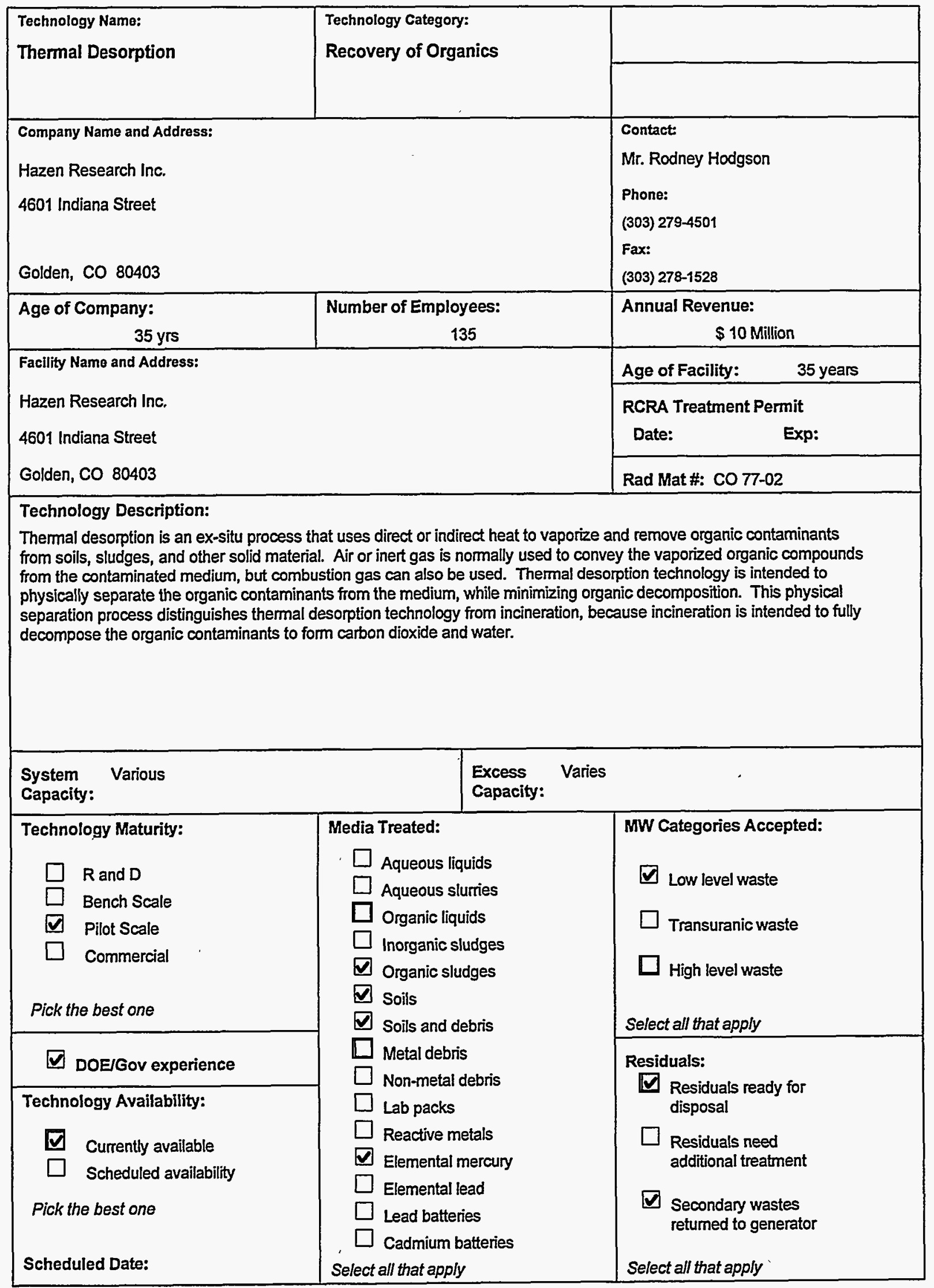


MIXED WASTE TREATABILITY STUDY TECHNOLOGY QUESTIONAIRE

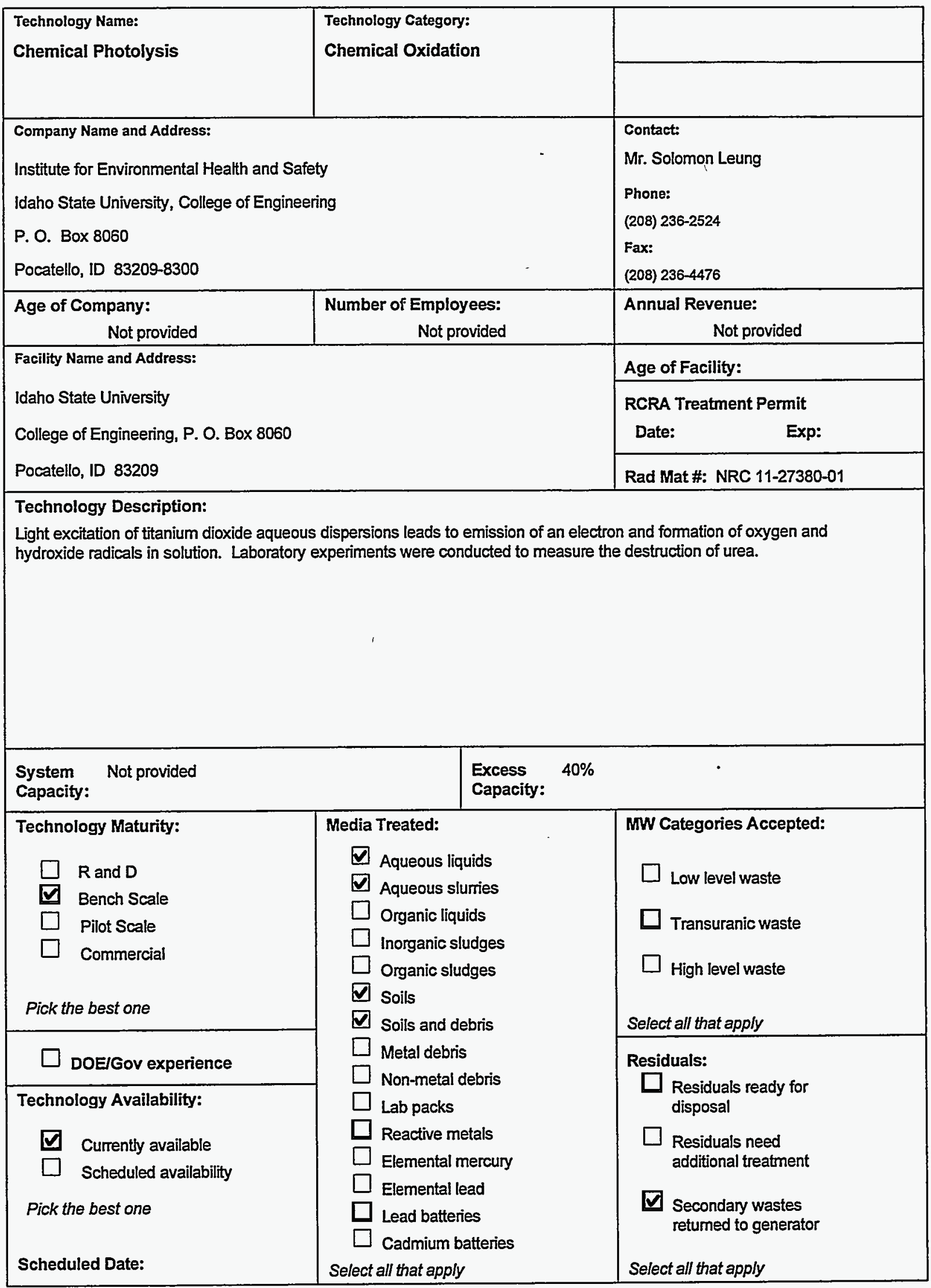




\section{MIXED WASTE TREATABILITY STUDY TECHNOLOGY QUESTIONAIRE}

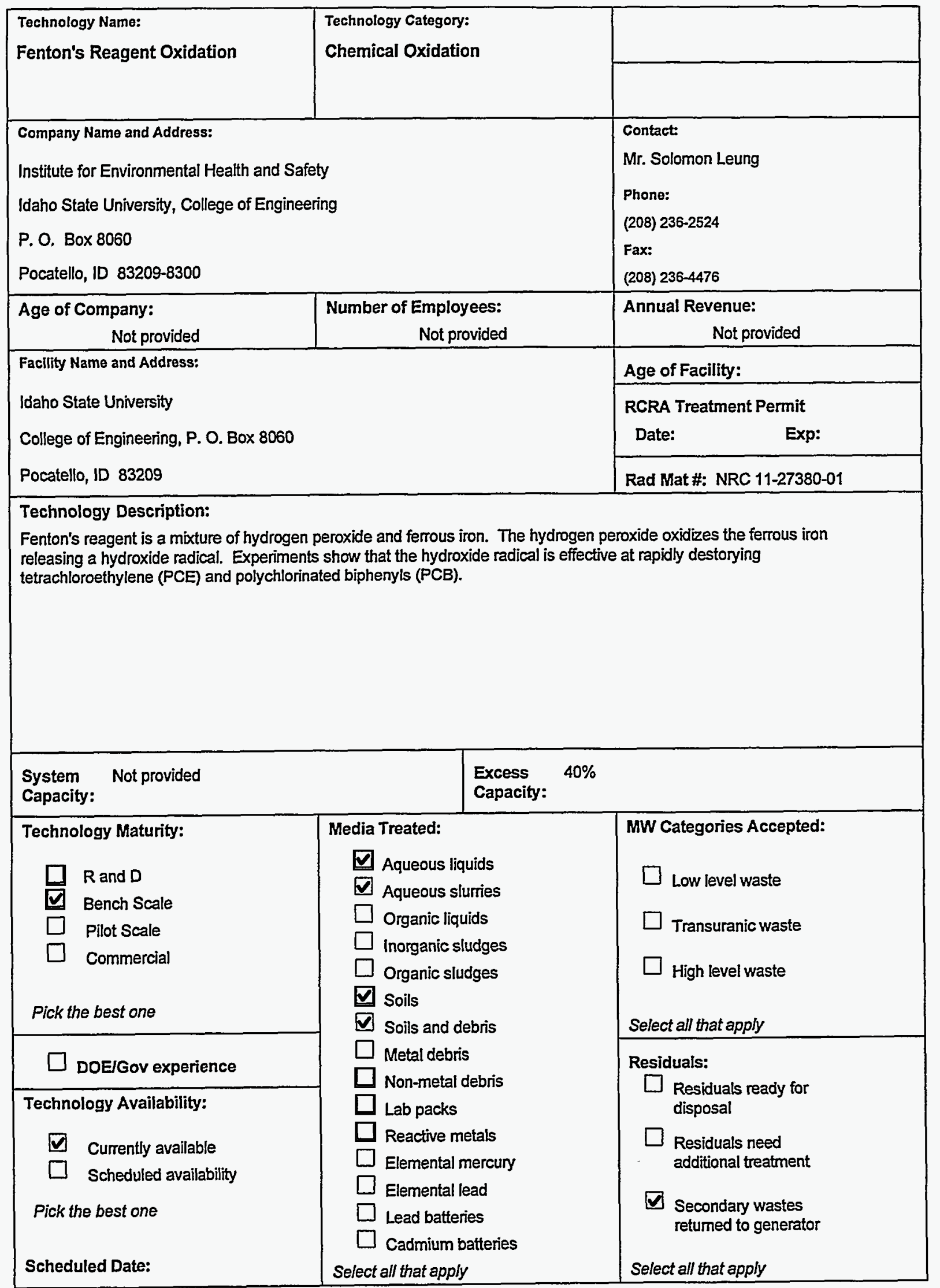


MIXED WASTE TREATABILITY STUDY TECHNOLOGY QUESTIONAIRE

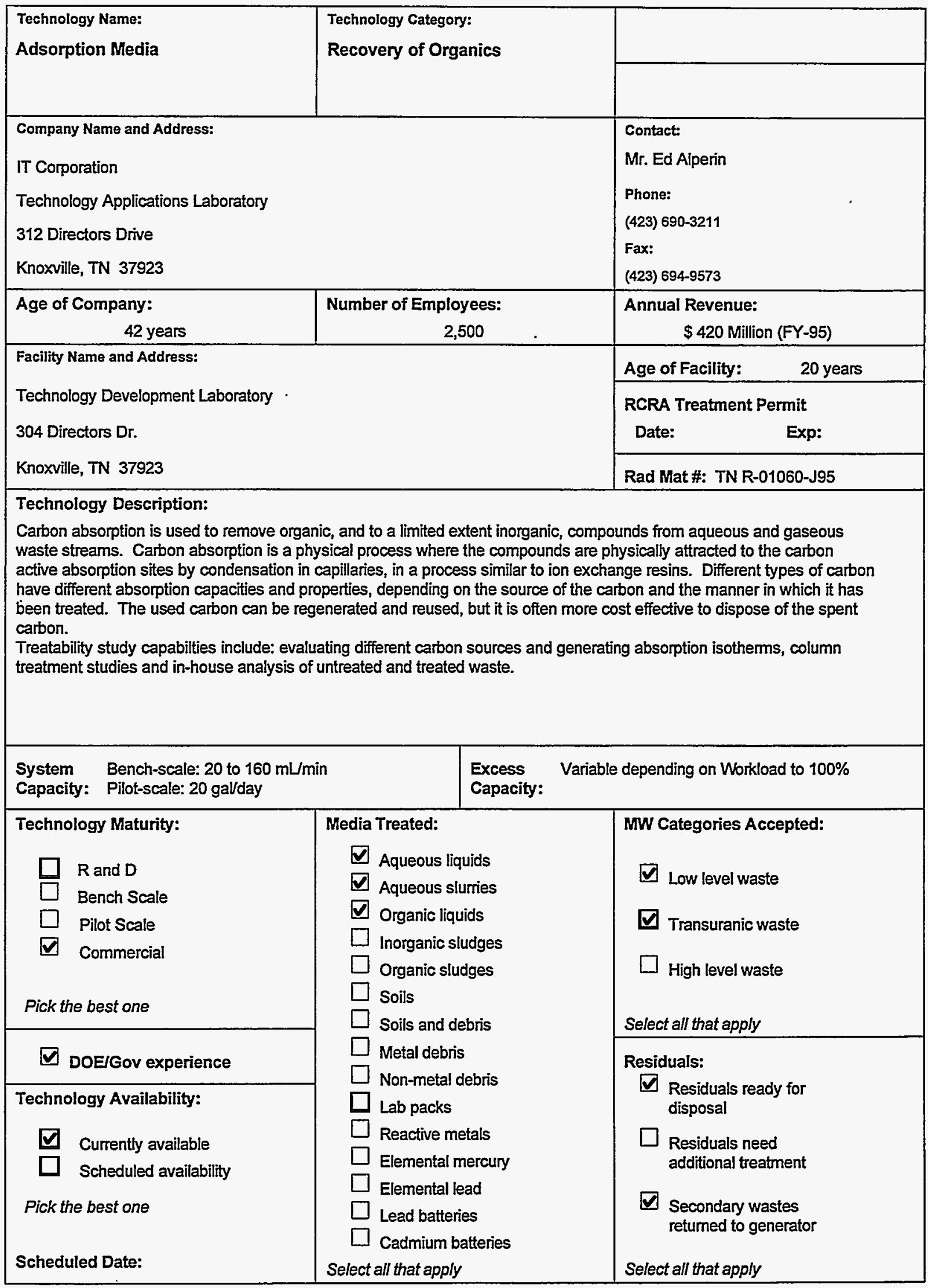




\section{MIXED WASTE TREATABILITY STUDY TECHNOLOGY QUESTIONAIRE}

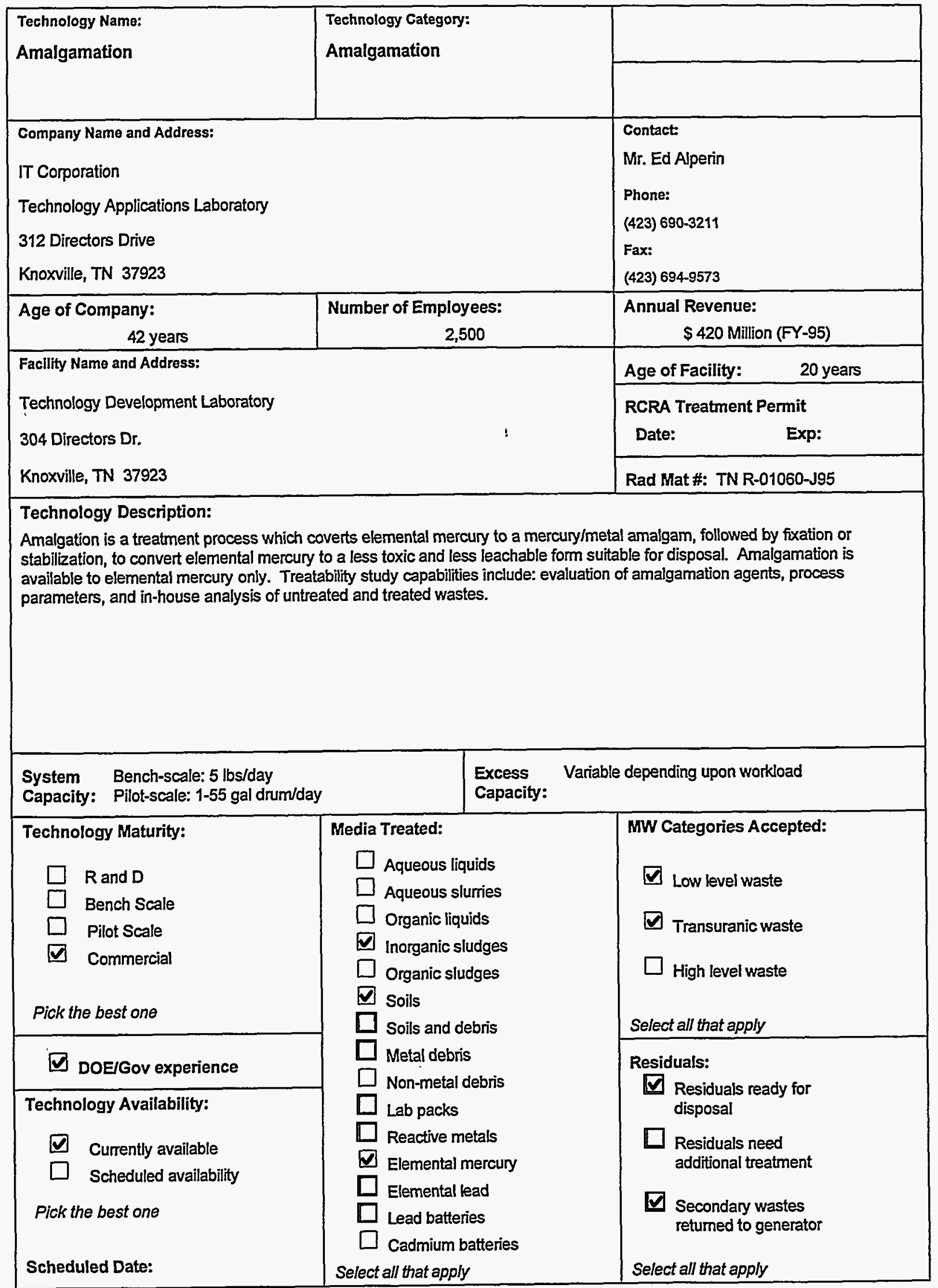


MIXED WASTE TREATABILITY STUDY TECHNOLOGY QUESTIONAIRE

\begin{tabular}{|c|c|c|}
\hline $\begin{array}{l}\text { Technology Name: } \\
\text { Base Catalyzed } \\
\text { Decomposition (BCD) }\end{array}$ & $\begin{array}{l}\text { Technology Category: } \\
\text { Organic Destruction }\end{array}$ & \\
\hline \multicolumn{2}{|l|}{$\begin{array}{l}\text { Company Name and Address: } \\
\text { IT Corporation } \\
\text { Technology Applications Laboratory } \\
312 \text { Directors Drive } \\
\text { Knoxville, TN } 37923\end{array}$} & $\begin{array}{l}\text { Contact: } \\
\text { Mr. Ed Alperin } \\
\text { Phone: } \\
\text { (423) } 690-3211 \\
\text { Fax: } \\
\text { (423) } 694-9573\end{array}$ \\
\hline $\begin{array}{l}\text { Age of Company: } \\
\qquad 42 \text { years }\end{array}$ & $\begin{array}{r}\text { Number of Employees: } \\
2,500 \\
\end{array}$ & $\begin{array}{l}\text { Annual Revenue: } \\
\text { \$ } 420 \text { Million (FY-95) }\end{array}$ \\
\hline \multirow{3}{*}{\multicolumn{2}{|c|}{$\begin{array}{l}\text { Facility Name and Address: } \\
\text { Technology Development Laboratory } \\
304 \text { Directors Dr. }\end{array}$}} & Age of Facility: \\
\hline & & $\begin{array}{l}\text { RCRA Treatment Permit } \\
\text { Date: }\end{array}$ \\
\hline & & Rad Mat \#: TN R-01060-J95 \\
\hline \multicolumn{3}{|c|}{$\begin{array}{l}\text { Technology Description: } \\
\text { Destruction of hazardous organic chlorinated compounds such as PCP, PCBs, and PCDDs/PCDFs is accomplished using } \\
\text { a patented EPA chemical process, which uses petroleum stocks as solvent, a catalyst, sodium hydroxide and the } \\
\text { application of heat. The reaction is usually conducted at } 300 \text { to } 350 \text { degrees C. The reaction displaces the chlorine atoms } \\
\text { in the hazardous compounds with a hydrogen atom producing nontoxic unchlorinated compounds and chloride salts as } \\
\text { byproducts. IT has experience using the process to destroy PCB compounds in low temperature thermal desorption } \\
\text { condensates. Treatability study capabilities include: evaluation of batch BCD process conditions and effectiveness, and in- } \\
\text { house analysis of treated and untreated waste as well as special analytical capabilities to analyze for by products. }\end{array}$} \\
\hline $\begin{array}{ll}\text { System } & \text { Bench-scale: } 2 \text { liter/batch } \\
\text { Capacity: } & \text { Pilot-scale: } 10 \text { gal/batch }\end{array}$ & $\begin{array}{l}\text { Excess } \\
\text { Capacity }\end{array}$ & depending on workload \\
\hline $\begin{array}{c}\text { Technology Maturity: } \\
\square \quad \text { R and D } \\
\square \quad \text { Bench Scale } \\
\square \quad \text { Pilot Scale } \\
\square \quad \text { Commercial } \\
\text { Pick the best one }\end{array}$ & $\begin{array}{l}\text { Media Treated: } \\
\square \text { Aqueous liquids } \\
\square \text { Aqueous slurries } \\
\square \text { Organic liquids } \\
\square \text { Inorganic sludges } \\
\square \text { Organic sludges } \\
\square \text { Soils } \\
\square \text { Soils and debris }\end{array}$ & $\begin{array}{l}\text { MW Categories Accepted: } \\
\square \text { Low level waste } \\
\square \text { Transuranic waste } \\
\square \text { High level waste } \\
\text { Select all that apply }\end{array}$ \\
\hline$\nabla$ DOE/Gov experience & $\square$ Metal debris & Residuals: \\
\hline $\begin{array}{l}\text { Technology Availability: } \\
\qquad \begin{array}{l}\square \\
\square\end{array} \\
\text { Schrently available } \\
\text { Pick the best one } \\
\text { Scheduled availability } \\
\end{array}$ & $\begin{array}{l}\square \text { Lab packs } \\
\square \text { Reactive metals } \\
\square \text { Elemental mercury } \\
\square \text { Elemental lead } \\
\square \text { Lead batteries } \\
\square \text { Cadmium batteries } \\
\text { Select all that apply }\end{array}$ & $\begin{array}{l}\text { Residuals ready for } \\
\text { disposal } \\
\square \text { Residuals need } \\
\text { additional treatment } \\
\square \text { Secondary wastes } \\
\text { retumed to generator } \\
\text { Select all that apply }\end{array}$ \\
\hline
\end{tabular}


MIXED WASTE TREATABILITY STUDY TECHNOLOGY QUESTIONAIRE

\begin{tabular}{|c|c|c|}
\hline $\begin{array}{l}\text { Technology Name: } \\
\text { Chemical Dehalogenation }\end{array}$ & $\begin{array}{l}\text { Technology Category: } \\
\text { Organic Destruction }\end{array}$ & \\
\hline \multicolumn{2}{|l|}{$\begin{array}{l}\text { Company Name and Address: } \\
\text { IT Corporation } \\
\text { Technology Applications Laboratory } \\
312 \text { Directors Drive } \\
\text { Knoxville, TN } 37923\end{array}$} & $\begin{array}{l}\text { Contact: } \\
\text { Mr. Ed Alperin } \\
\text { Phone: } \\
\text { (423) 690-3211 } \\
\text { Fax: } \\
\text { (423) 694-9573 }\end{array}$ \\
\hline $\begin{array}{r}\text { Age of Company: } \\
42 \text { years }\end{array}$ & $\begin{array}{r}\text { Number of Employees: } \\
2,500 \\
\end{array}$ & $\begin{array}{l}\text { Annual Revenue: } \\
\text { \$ } 420 \text { Million (FY-95) } \\
\end{array}$ \\
\hline \multirow{3}{*}{\multicolumn{2}{|c|}{$\begin{array}{l}\text { Facility Name and Address: } \\
\text { Technology Development Laboratory } \\
304 \text { Directors Dr. } \\
\text { Knoxville, TN } 37923\end{array}$}} & Age of Facility: \\
\hline & & $\begin{array}{l}\text { RCRA Treatment Permit } \\
\text { Date: } \quad \text { Exp: }\end{array}$ \\
\hline & & Rad Mat \#: TN R-01060-J95 \\
\hline \multicolumn{3}{|c|}{$\begin{array}{l}\text { Technology Description: } \\
\text { Destruction of hazardous organic chlorinated compound such as pesticides, PCBs and PCDDs/PCDFs in organic or solid } \\
\text { wastes is accomplished using chemical reagents and in some cases, the application of heat. The reagents used are } \\
\text { typically basic compounds which supply a nucleophilic species, such as hydroxide, to displace the chloride atoms in the } \\
\text { hazardous compound. The products of reaction are typically nucleophilic substituted compounds and chloride salts. The } \\
\text { substituted compounds are much less or nontoxic, and in many cases are water soluble so they can be removed by } \\
\text { extraction. IT has extensive experience studying reagent destruction of PCBs and PCDDs/PCDFs on solid surfaces and in } \\
\text { transformer oils and organic wastes. Treatability study capabilties include: evaluation of chemcial dehalogenation reagents } \\
\text { and processes, evaluation of secondary waste treatment options, and analysis of treated and untreated waste as well as } \\
\text { special analytical capabilties to analyze for byproducts. ITS TOSCA permit prohibits retum of TOSCA regulated materials } \\
\text { to the generator. Special arrangements must be made for mixed waste. }\end{array}$} \\
\hline $\begin{array}{l}\text { System Bench-scale: } 10 \mathrm{~L} \text { batch } \\
\text { Capacity: Pilot-scale: } 10 \text { gallon batch }\end{array}$ & $\begin{array}{l}\text { Excess } \\
\text { Capacity: }\end{array}$ & depending on workload \\
\hline $\begin{array}{c}\text { Technology Maturity: } \\
\square \text { R and D } \\
\square \text { Bench Scale } \\
\square \text { Pilot Scale } \\
\square \text { Commercial } \\
\text { Pick the best one }\end{array}$ & $\begin{array}{l}\text { Media Treated: } \\
\square \text { Aqueous liquids } \\
\square \text { Aqueous slurries } \\
\square \text { Organic liquids } \\
\square \text { Inorganic sludges } \\
\square \text { Organic sludges } \\
\square \text { Soils } \\
\square \text { Soils and debris }\end{array}$ & $\begin{array}{l}\text { MW Categories Accepted: } \\
\square \text { Low level waste } \\
\square \text { Transuranic waste } \\
\square \text { High level waste } \\
\text { Select all that apply }\end{array}$ \\
\hline$\square$ DOE/Gov experience & $\begin{array}{l}\square \text { Metal debris } \\
\square \text { Non-metal debris }\end{array}$ & Residuals: \\
\hline $\begin{array}{l}\text { Technology Availability: } \\
\qquad \begin{array}{l}\square \text { Currently available } \\
\square \text { Scheduled availability }\end{array} \\
\text { Pick the best one } \\
\text { Scheduled Date: }\end{array}$ & $\begin{array}{l}\square \text { Lab packs } \\
\square \text { Reactive metals } \\
\square \text { Elemental mercury } \\
\square \text { Elemental lead } \\
\square \text { Lead batteries } \\
\square \text { Cadmium batteries } \\
\text { Select all that apply }\end{array}$ & $\begin{array}{l}\text { disposal } \\
\nabla \text { Residuals need } \\
\text { additional treatment } \\
\text { Secondary wastes } \\
\text { retumed to generator } \\
\text { Select all that apply }\end{array}$ \\
\hline
\end{tabular}


MIXED WASTE TREATABILITY STUDY TECHNOLOGY QUESTIONAIRE

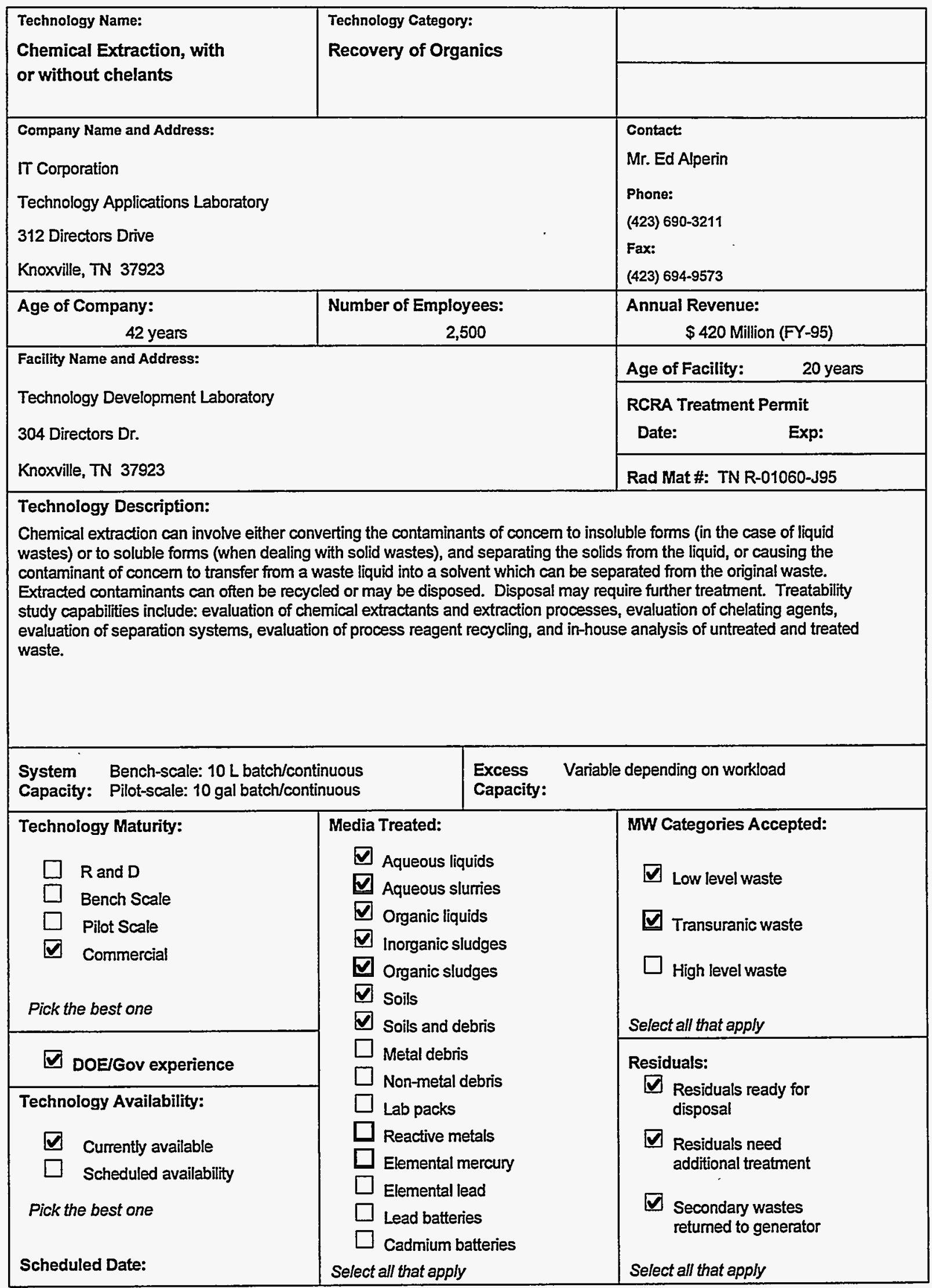


MIXED WASTE TREATABILITY STUDY TECHNOLOGY QUESTIONAIRE

\begin{tabular}{|c|c|c|c|}
\hline $\begin{array}{l}\text { Technology Name: } \\
\text { Deactivation }\end{array}$ & \multicolumn{2}{|c|}{$\begin{array}{l}\text { Technology Category: } \\
\text { Deactivation }\end{array}$} & \\
\hline $\begin{array}{l}\text { Company Name and Address: } \\
\text { IT Corporation } \\
\text { Technology Applications Labora } \\
312 \text { Directors Drive } \\
\text { Knoxville, TN } 37923\end{array}$ & & & $\begin{array}{l}\text { Contact: } \\
\text { Mr. Ed Alperin } \\
\text { Phone: } \\
\text { (423) 690-3211 } \\
\text { Fax: } \\
(423) 694-9573\end{array}$ \\
\hline $\begin{array}{l}\text { Age of Company: } \\
\qquad 42 \text { years }\end{array}$ & \multicolumn{2}{|c|}{$\begin{array}{r}\text { Number of Employees: } \\
2,500 \\
\end{array}$} & $\begin{array}{l}\text { Annual Revenue: } \\
\qquad 420 \text { Million (FY-95) } \\
\end{array}$ \\
\hline \multicolumn{3}{|c|}{$\begin{array}{l}\text { Facility Name and Address: } \\
\text { Technology Development Laboratory }\end{array}$} & Age of Facility: $\quad 20$ years \\
\hline \multicolumn{3}{|c|}{$\begin{array}{l}\text { Technology Development Laboratory } \\
304 \text { Directors Dr. }\end{array}$} & $\begin{array}{l}\text { RCRA Treatment Permit } \\
\text { Date: } \quad \text { Exp: }\end{array}$ \\
\hline \multicolumn{3}{|c|}{$\begin{array}{l}304 \text { Directors Dr. } \\
\text { Knoxville, TN } 37923\end{array}$} & Rad Mat \#: TN R-01060-J95 \\
\hline \multicolumn{4}{|c|}{$\begin{array}{l}\text { Technology Description: } \\
\text { Deactivation is any acceptable process which will reduce a waste's hazardous property to meet Land Disposal } \\
\text { Requirements. Hazards such as igniteability, corrosivity, toxicity or reactivity can be treated by deactivation. There are } \\
\text { many treatment technologies that may be applied to deactiviation, including pH adjustments, precipitation, oxidation, to } \\
\text { name a few. Treatability study capabilities include: evaluation of treatment alternatives, bench-and pilot-scale testing of } \\
\text { treatment alternatives, coupling with other treatment alternatives, to provide a complete treatment process, and in-house } \\
\text { analysis of untreated and treated waste. }\end{array}$} \\
\hline \multicolumn{2}{|c|}{$\begin{array}{ll}\text { System } & \text { Bench-scale: } 1 \text { L or } 4 \text { lbs per day } \\
\text { Capacity: } & \text { Pilot-scale: } 10 \text { L or } 40 \text { lbs per day }\end{array}$} & \multicolumn{2}{|c|}{$\begin{array}{l}\text { Excess Varialbe depending on workload } \\
\text { Capacity: }\end{array}$} \\
\hline $\begin{array}{l}\text { Technology Maturity: } \\
\square \text { R and D } \\
\square \text { Bench Scale } \\
\square \text { Pilot Scale } \\
\square \quad \text { Commercial } \\
\text { Pick the best one }\end{array}$ & \multirow{3}{*}{\multicolumn{2}{|c|}{$\begin{array}{l}\text { Media Treated: } \\
\square \text { Aqueous liquids } \\
\square \text { Aqueous slumies } \\
\square \text { Organic liquids } \\
\square \text { Inorganic sludges } \\
\square \text { Organic sludges } \\
\square \text { Soils } \\
\square \text { Soils and debris } \\
\square \text { Metal debris } \\
\square \text { Non-metal debris } \\
\square \text { Lab packs } \\
\square \text { Reactive metals } \\
\square \text { Elemental mercury } \\
\square \text { Elemental lead } \\
\square \text { Lead batteries } \\
\square \text { Cadmium batteries } \\
\text { Select all that apply }\end{array}$}} & $\begin{array}{l}\text { MW Categories Accepted: } \\
\square \text { Low level waste } \\
\square \text { Transuranic waste } \\
\square \text { High level waste } \\
\text { Select all that apply }\end{array}$ \\
\hline$\square$ DOE/Gov experience & & & Residuals: \\
\hline $\begin{array}{l}\text { Technology Availability: } \\
\square \text { Currently available } \\
\square \text { Scheduled availability } \\
\text { Pick the best one } \\
\text { Scheduled Date: }\end{array}$ & & & $\begin{array}{l}\text { Residuals need } \\
\text { additional treatment } \\
\text { disposal } \\
\text { Secondary wastes } \\
\text { retumed to generator } \\
\text { Select all that apply }\end{array}$ \\
\hline
\end{tabular}




\section{MIXED WASTE TREATABILITY STUDY TECHNOLOGY QUESTIONAIRE}

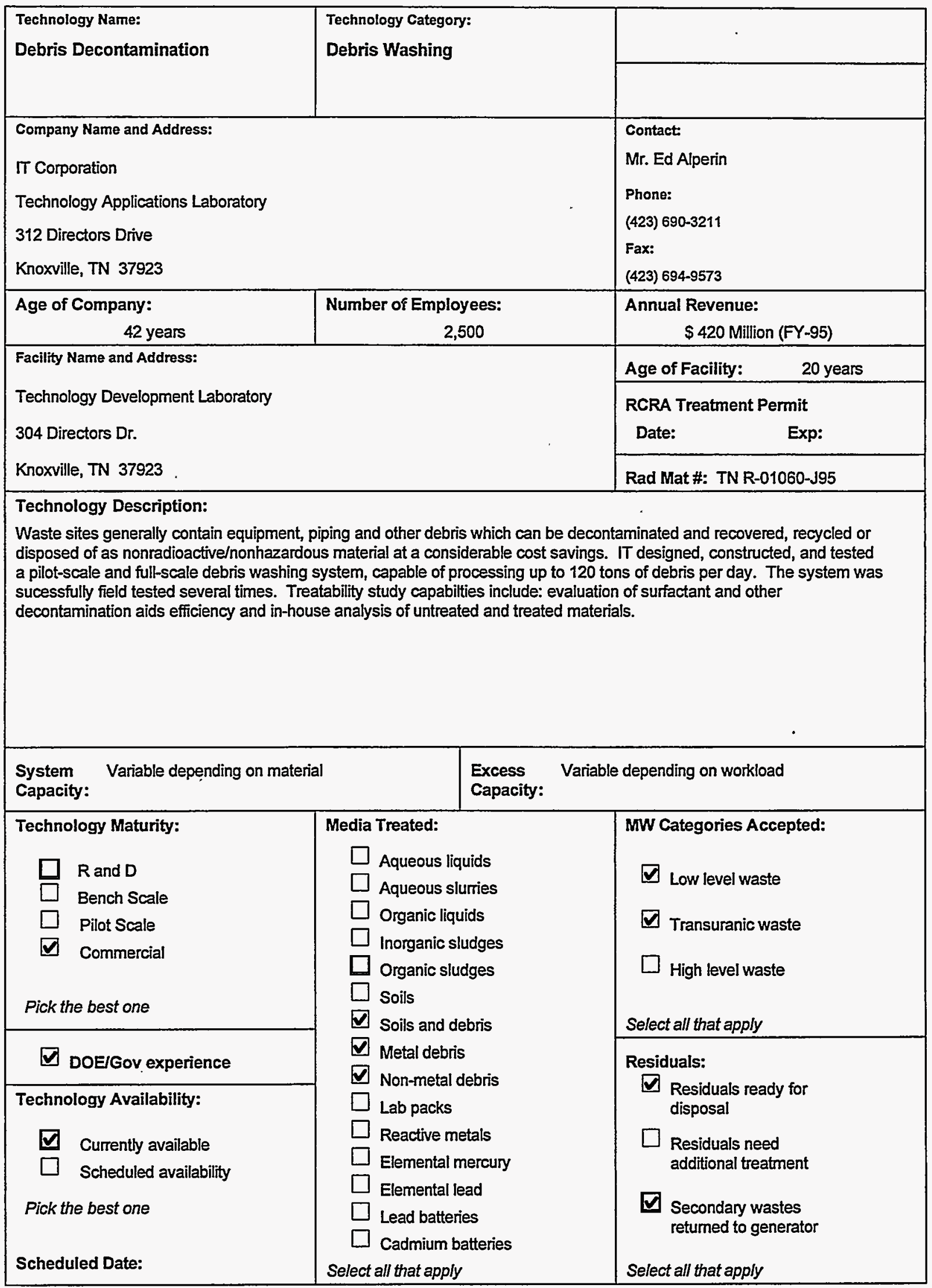




\section{MIXED WASTE TREATABILITY STUDY TECHNOLOGY QUESTIONAIRE}

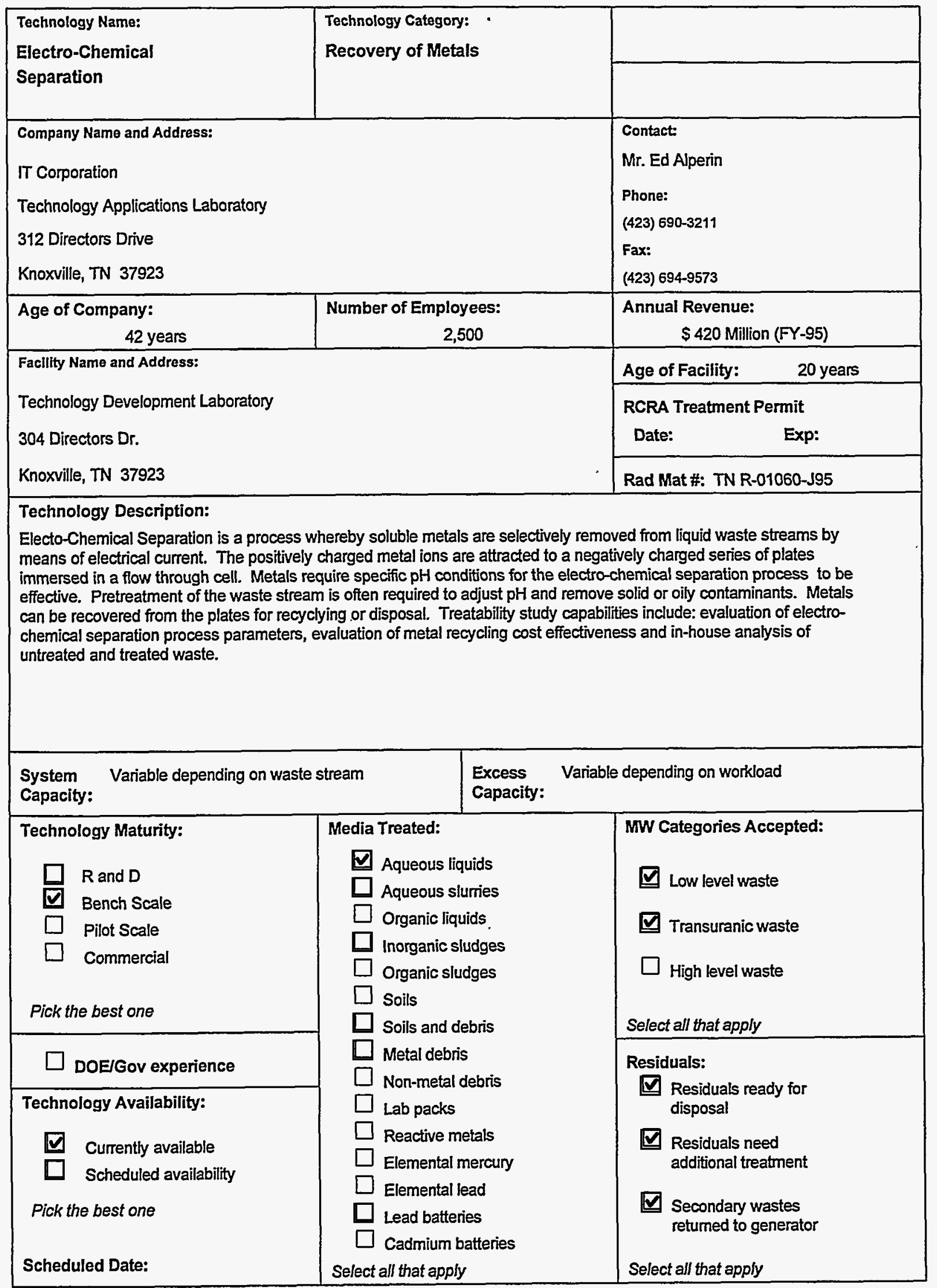


MIXED WASTE TREATABILITY STUDY TECHNOLOGY QUESTIONAIRE

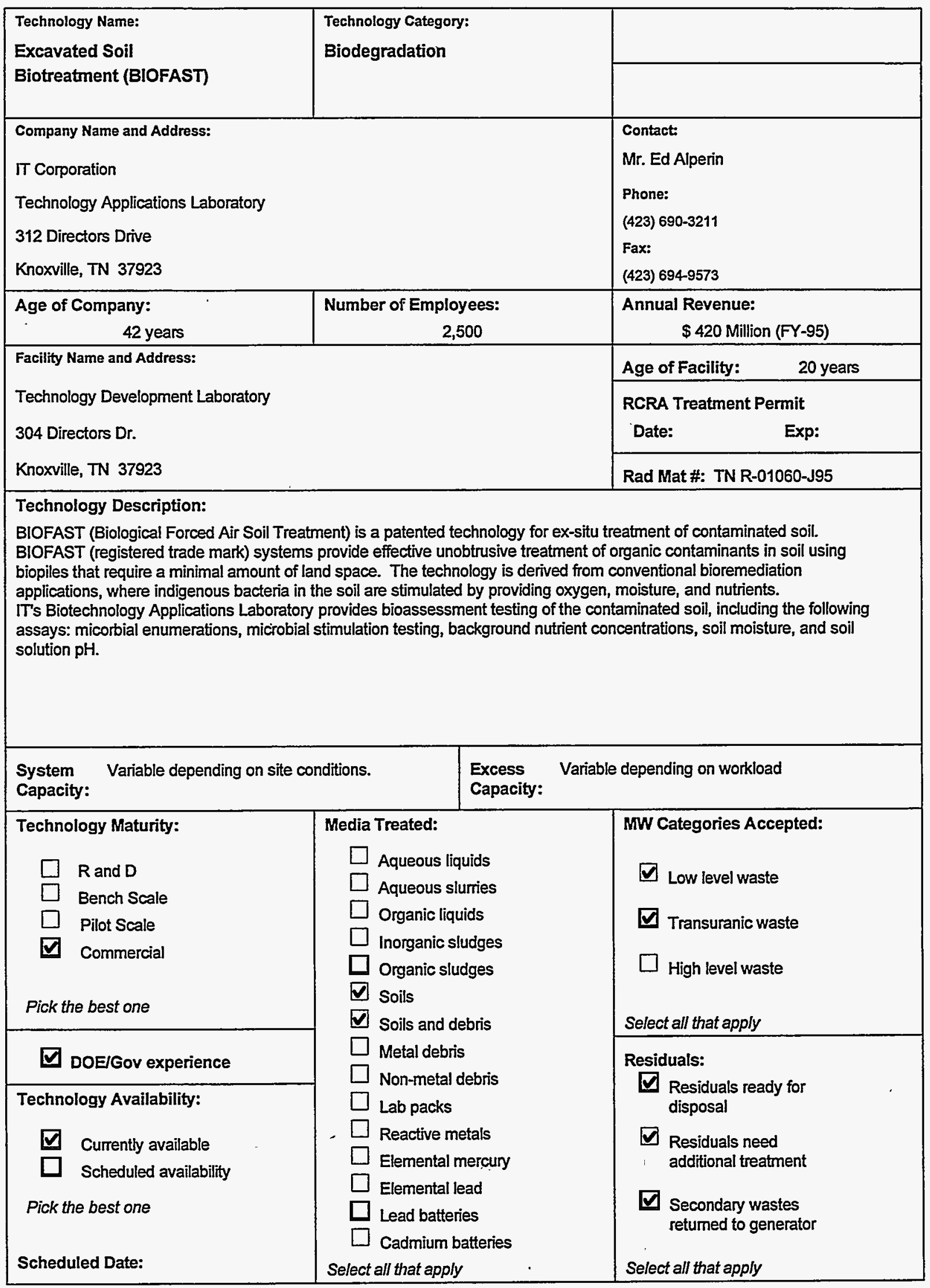


MIXED WASTE TREATABILITY STUDY TECHNOLOGY QUESTIONAIRE

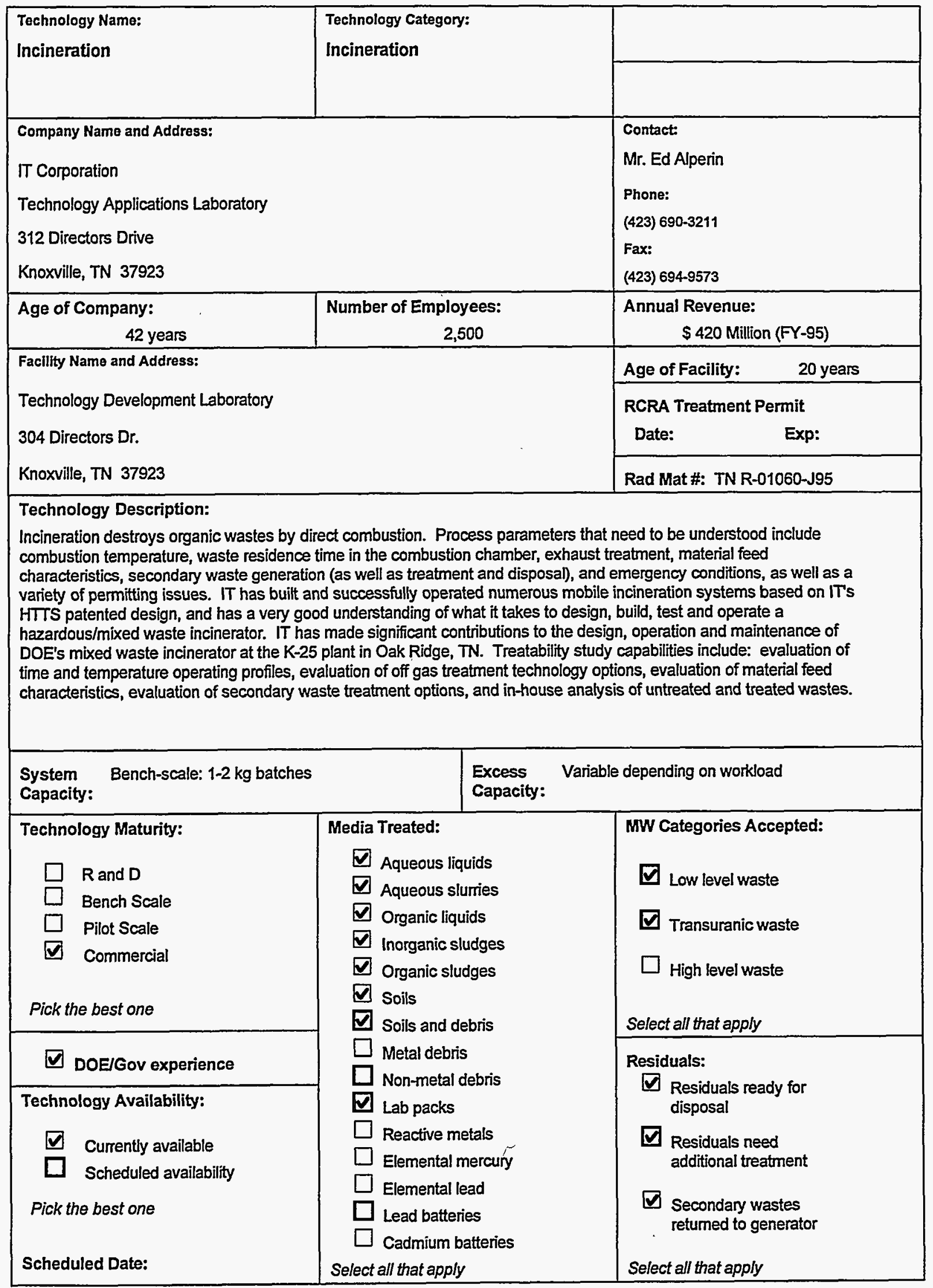


MIXED WASTE TREATABILITY STUDY TECHNOLOGY QUESTIONAIRE






\section{MIXED WASTE TREATABILITY STUDY TECHNOLOGY QUESTIONAIRE}

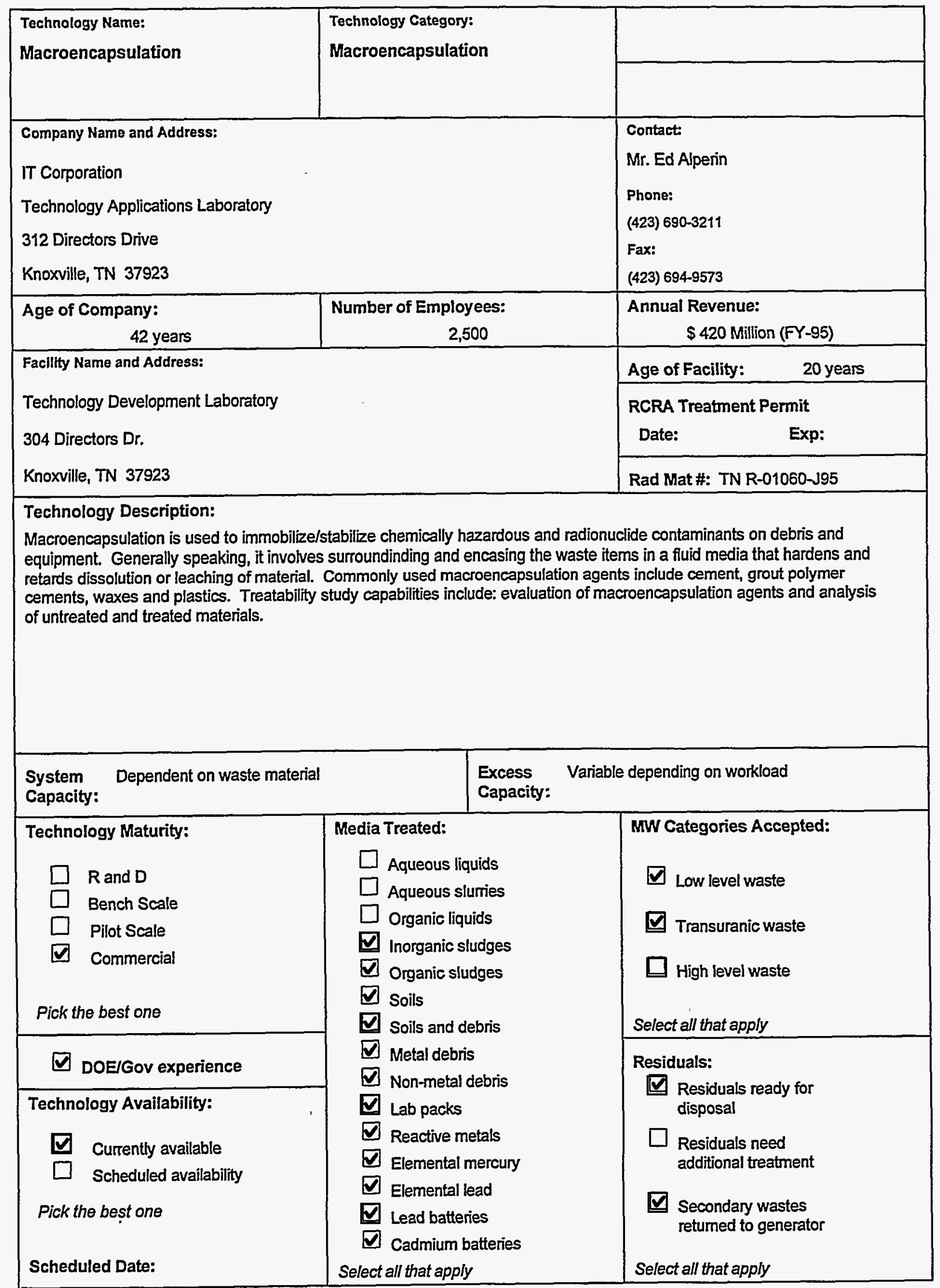


MIXED WASTE TREATABILITY STUDY TECHNOLOGY QUESTIONAIRE






\section{MIXED WASTE TREATABILITY STUDY TECHNOLOGY QUESTIONAIRE}

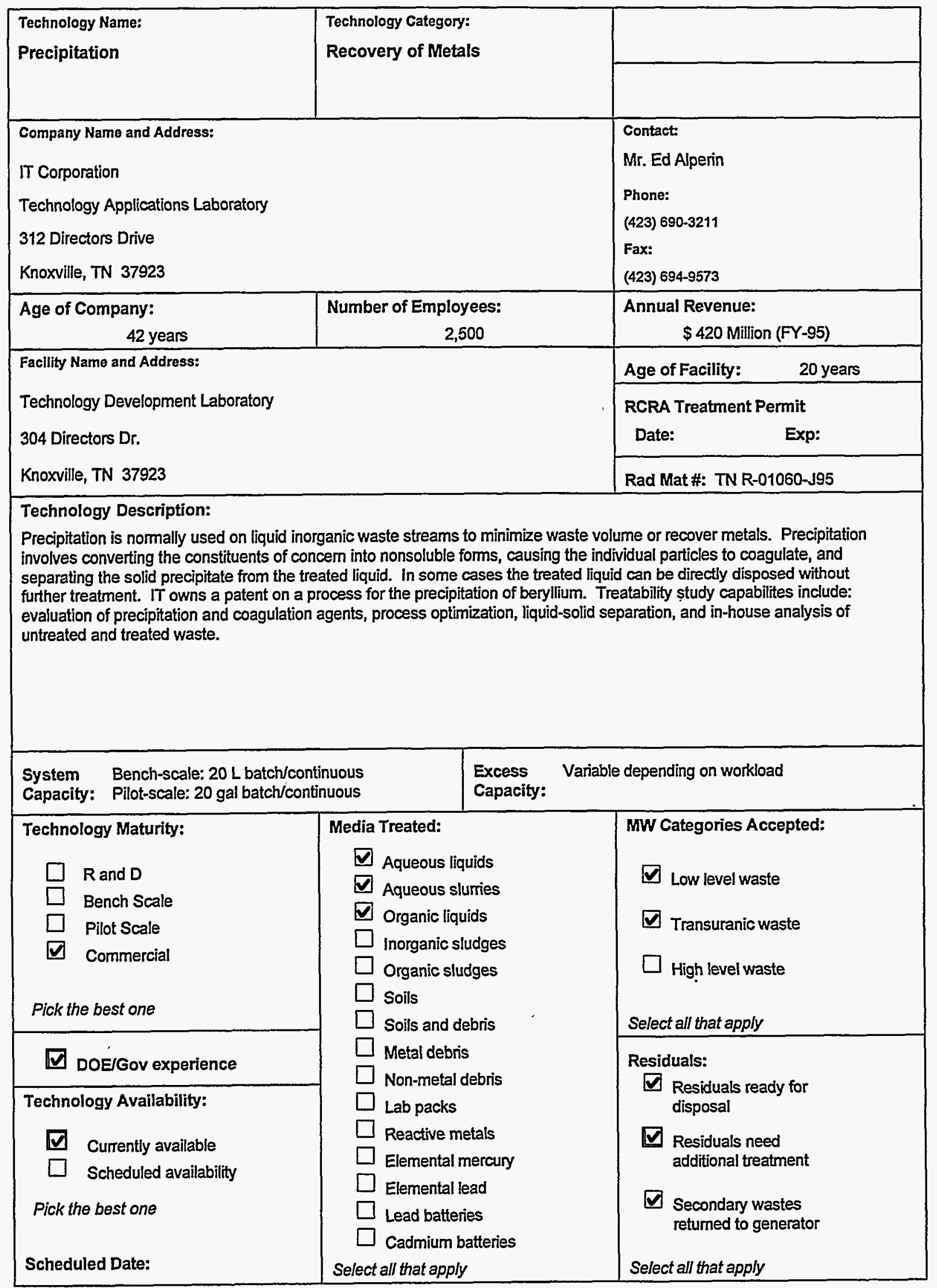


MIXED WASTE TREATABILITY STUDY TECHNOLOGY QUESTIONAIRE

\begin{tabular}{|c|c|c|}
\hline $\begin{array}{l}\text { Technology Name: } \\
\text { Reverse Osmosis }\end{array}$ & $\begin{array}{l}\text { Technology Category: } \\
\text { Recovery of Metals }\end{array}$ & \\
\hline \multicolumn{2}{|l|}{$\begin{array}{l}\text { Company Name and Address: } \\
\text { IT Corporation } \\
\text { Technology Applications Laboratory } \\
312 \text { Directors Drive } \\
\text { Knoxville, TN } 37923\end{array}$} & $\begin{array}{l}\text { Contact: } \\
\text { Mr. Ed Alperin } \\
\text { Phone: } \\
\text { (423) 690-3211 } \\
\text { Fax: } \\
\text { (423) 694-9573 }\end{array}$ \\
\hline $\begin{array}{l}\text { Age of Company: } \\
\qquad 42 \text { years }\end{array}$ & $\begin{array}{r}\text { Number of Employees: } \\
2,500\end{array}$ & $\begin{array}{l}\text { Annual Revenue: } \\
\qquad \$ 420 \text { Million (FY-95) }\end{array}$ \\
\hline \multirow{3}{*}{\multicolumn{2}{|c|}{$\begin{array}{l}\text { Facility Name and Address: } \\
\text { Technology Development Laboratory } \\
304 \text { Directors Dr. } \\
\text { Knoxville, TN } 37923\end{array}$}} & Age of Facility: $\quad 20$ years \\
\hline & & $\begin{array}{l}\text { RCRA Treatment Permit } \\
\text { Date: }\end{array}$ \\
\hline & & Rad Mat \#: TN R-01060-J95 \\
\hline \multicolumn{3}{|c|}{$\begin{array}{l}\text { Technology Description: } \\
\text { Reverse osmosis is a process for obtaining purified water and a solute concentrate from an aqueous stream containing } \\
\text { soluble contaminants. The process uses a permeable membrane that can withstand pressure. On one side of the } \\
\text { membrane is the contaminated aqueous stream, and on the other side is the treated or purified water. A pressure } \\
\text { differential is created across the membrane due to the difference in solute concentration on either side of the membrane. } \\
\text { When pressure exceeding the pressure differential is applied to the side containing the solutes, water permeates across the } \\
\text { membrane to produce purified water until the osmotic pressure approaches that of the applied pressure. The solute } \\
\text { concentrate stream can then be used for the recovery of dissolved compounds. Treatability study capabilities include: } \\
\text { evaluation of treatment process, conditions and effectiveness and in-house analysis for organics as well as inorganics: } \\
\text { metal, cations and anions. }\end{array}$} \\
\hline $\begin{array}{ll}\text { System } & \text { Bench-scale: Variable } \\
\text { Capacity: } & \text { Pilot-scale: } 1 \text { to } 2 \text { gpm }\end{array}$ & $\begin{array}{l}\text { Excess } \\
\text { Capacity: }\end{array}$ & depending on workload \\
\hline $\begin{array}{l}\text { Technology Maturity: } \\
\square \quad \text { R and D } \\
\square \quad \text { Bench Scale } \\
\square \quad \text { Pilot Scale } \\
\square \quad \text { Commercial } \\
\text { Pick the best one }\end{array}$ & $\begin{array}{l}\text { Media Treated: } \\
\square \text { Aqueous liquids } \\
\square \text { Aqueous slurries } \\
\square \text { Organic liquids } \\
\square \text { Inorganic sludges } \\
\square \text { Organic sludges } \\
\square \text { Soils } \\
\square \text { Soils and debris }\end{array}$ & $\begin{array}{l}\text { MW Categories Accepted: } \\
\square \text { Low level waste } \\
\square \text { Transuranic waste } \\
\square \text { High level waste } \\
\text { Select all that apply }\end{array}$ \\
\hline$\square$ DOE/Gov experience & $\begin{array}{l}\square \text { Metal debris } \\
\square \text { Non-metal debris }\end{array}$ & Residuals: \\
\hline $\begin{array}{l}\text { Technology Availability: } \\
\qquad \begin{array}{l}\square \\
\text { Currently available }\end{array} \\
\text { Scheduled availability } \\
\text { Pick the best one } \\
\text { Scheduled Date: }\end{array}$ & $\begin{array}{l}\square \text { Lab packs } \\
\square \text { Reactive metals } \\
\square \text { Elemental mercury } \\
\square \text { Elemental lead } \\
\square \text { Lead batteries } \\
\square \text { Cadmium batteries } \\
\text { Select all that apply }\end{array}$ & $\begin{array}{l}\text { Residuals ready for } \\
\text { disposal } \\
\text { Residuals need } \\
\text { additional treatment } \\
\text { Secondary wastes } \\
\text { retumed to generator }\end{array}$ \\
\hline
\end{tabular}




\section{MIXED WASTE TREATABILITY STUDY TECHNOLOGY QUESTIONAIRE}

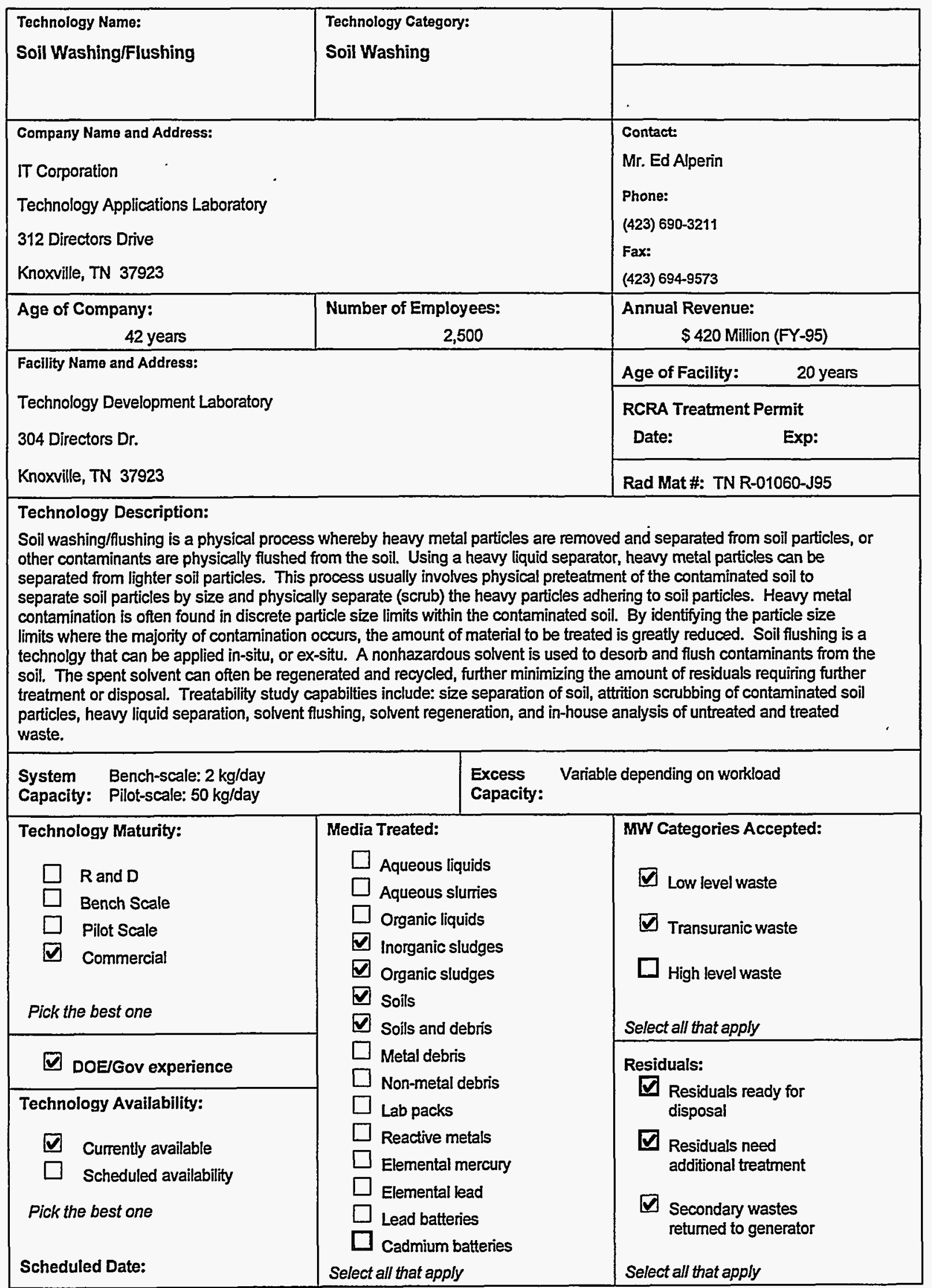


MIXED WASTE TREATABILITY STUDY TECHNOLOGY QUESTIONAIRE

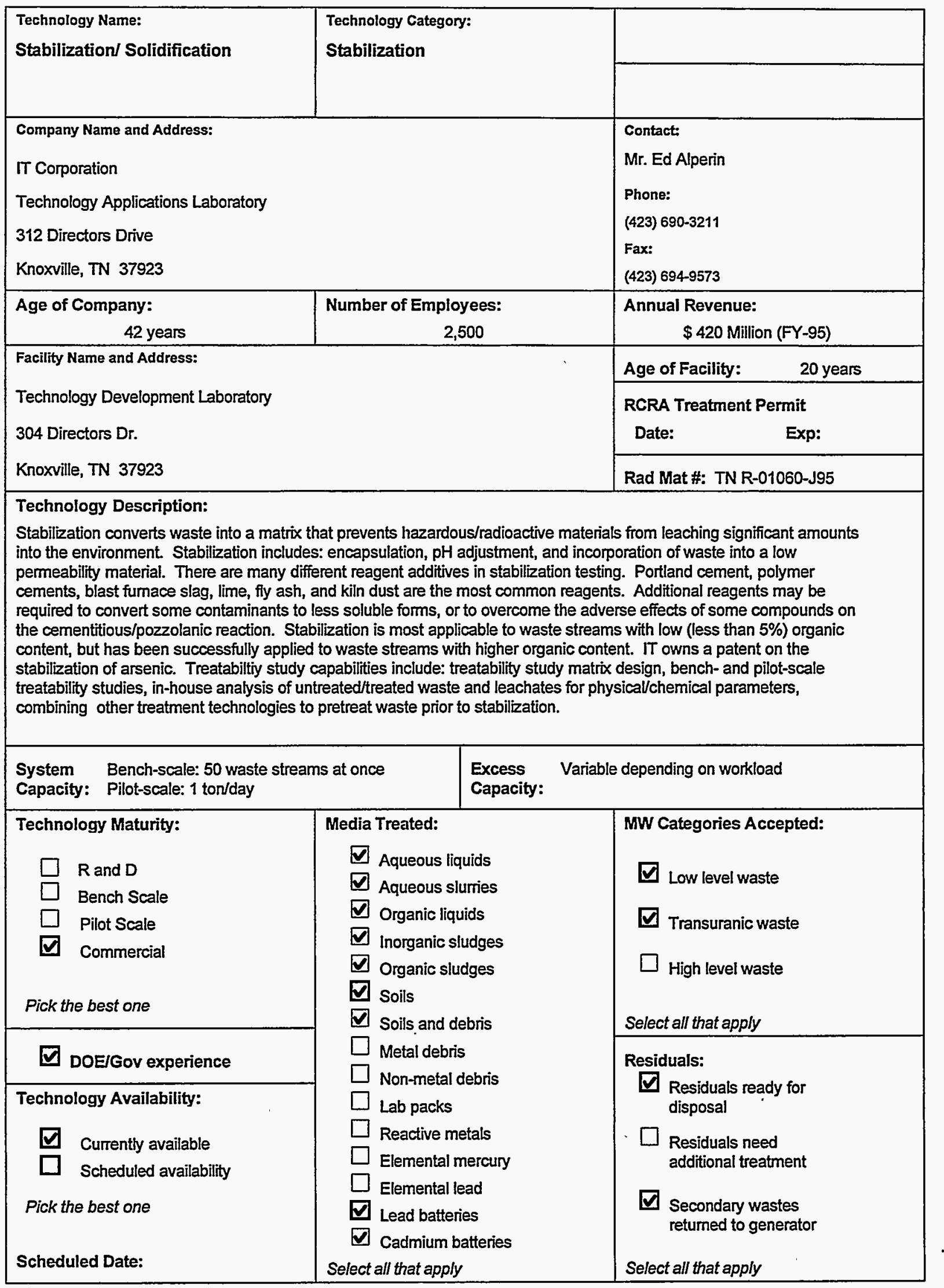


MIXED WASTE TREATABILITY STUDY TECHNOLOGY QUESTIONAIRE

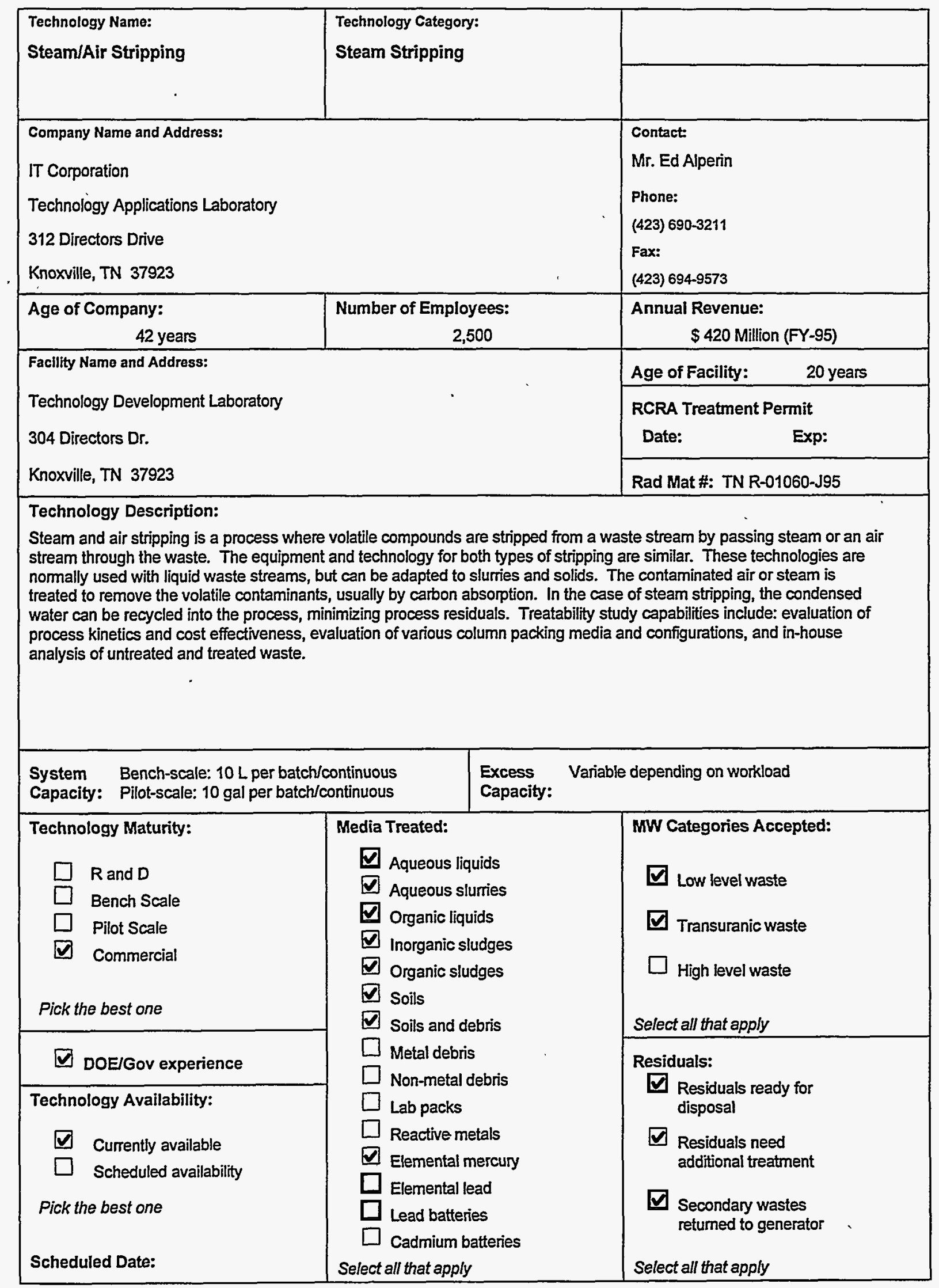


MIXED WASTE TREATABILITY STUDY TECHNOLOGY QUESTIONAIRE

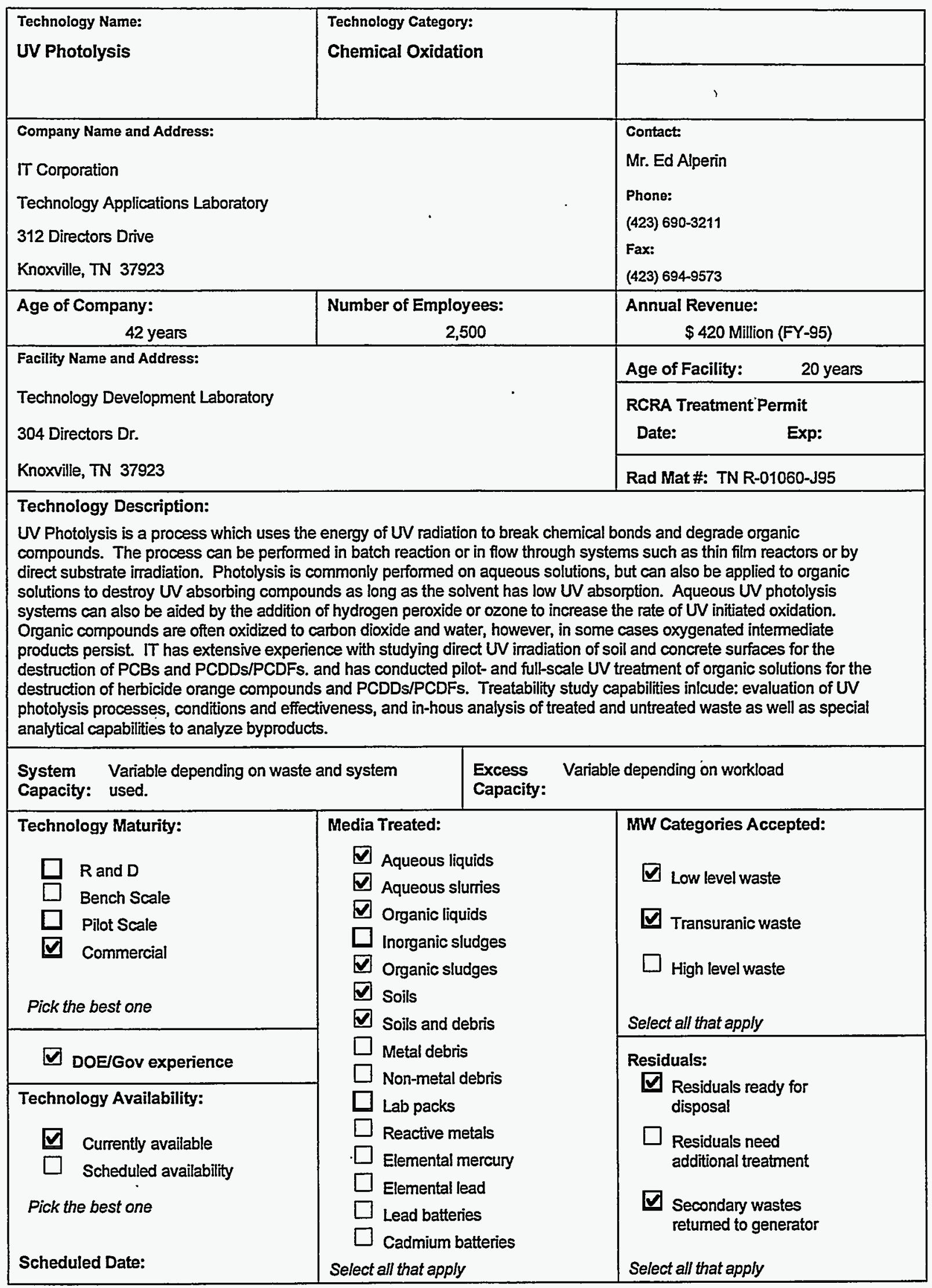




\section{MIXED WASTE TREATABILITY STUDY TECHNOLOGY QUESTIONAIRE}

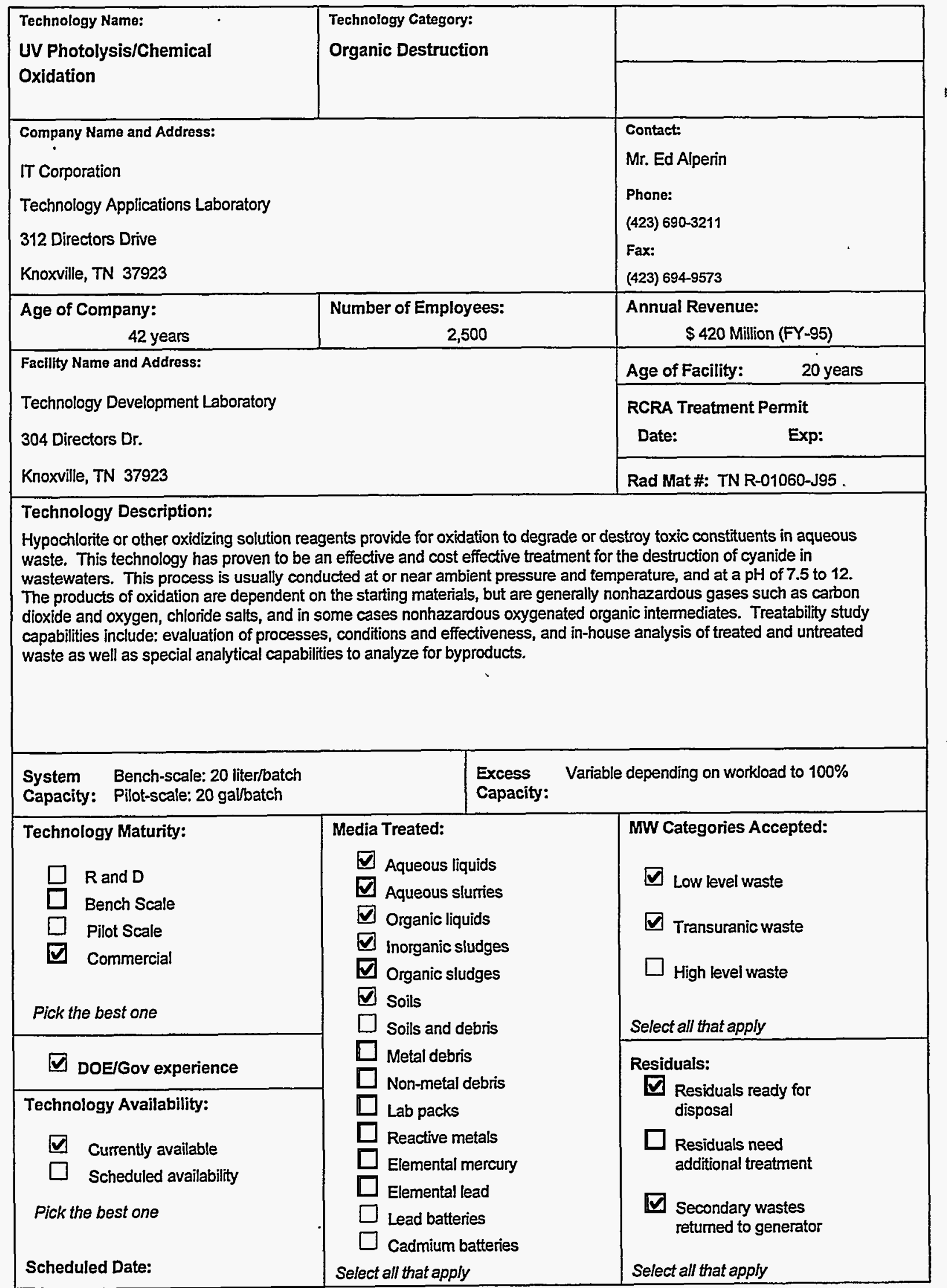


MIXED WASTE TREATABILITY STUDY TECHNOLOGY QUESTIONAIRE

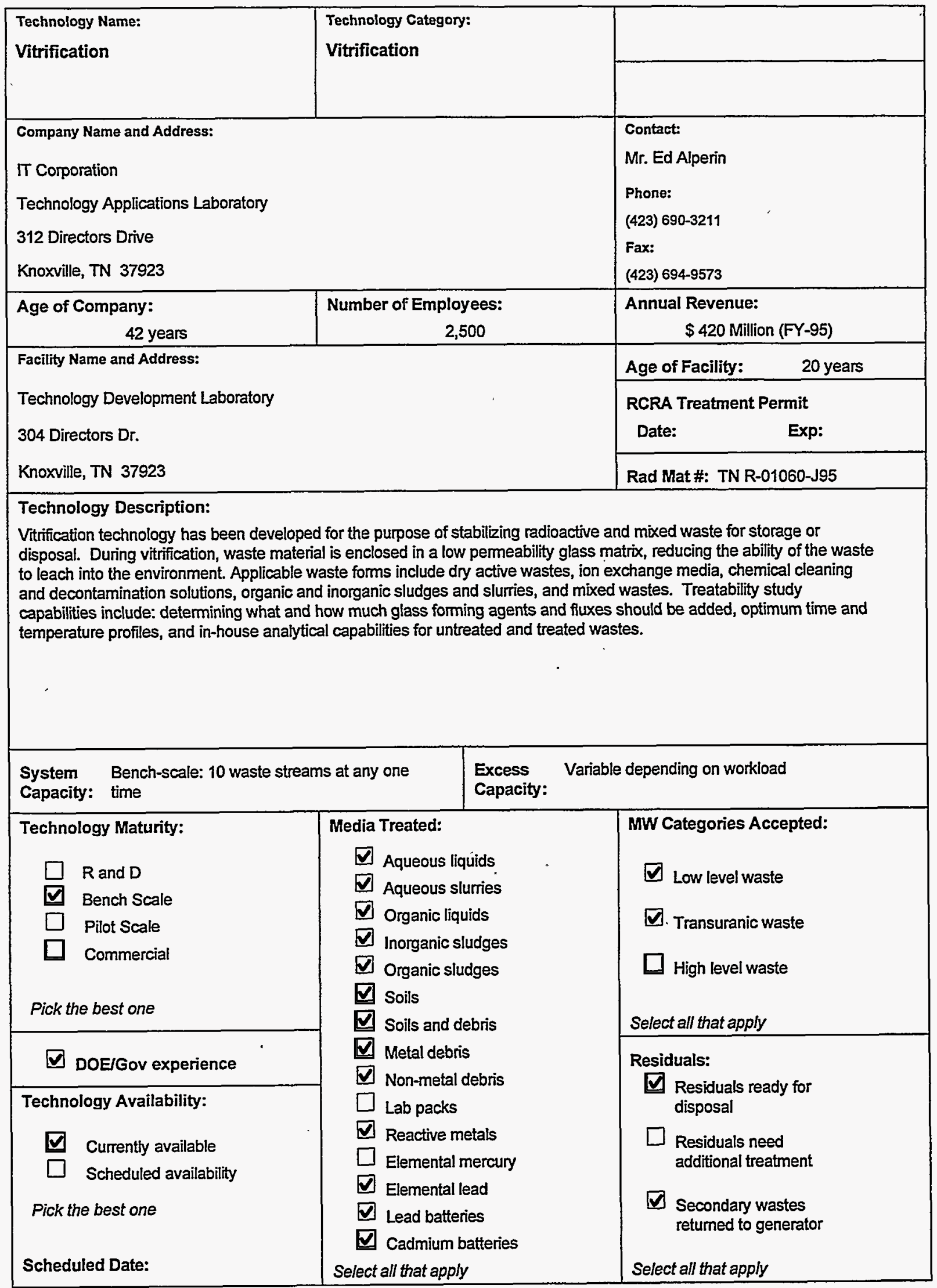


MIXED WASTE TREATABILITY STUDY TECHNOLOGY QUESTIONAIRE

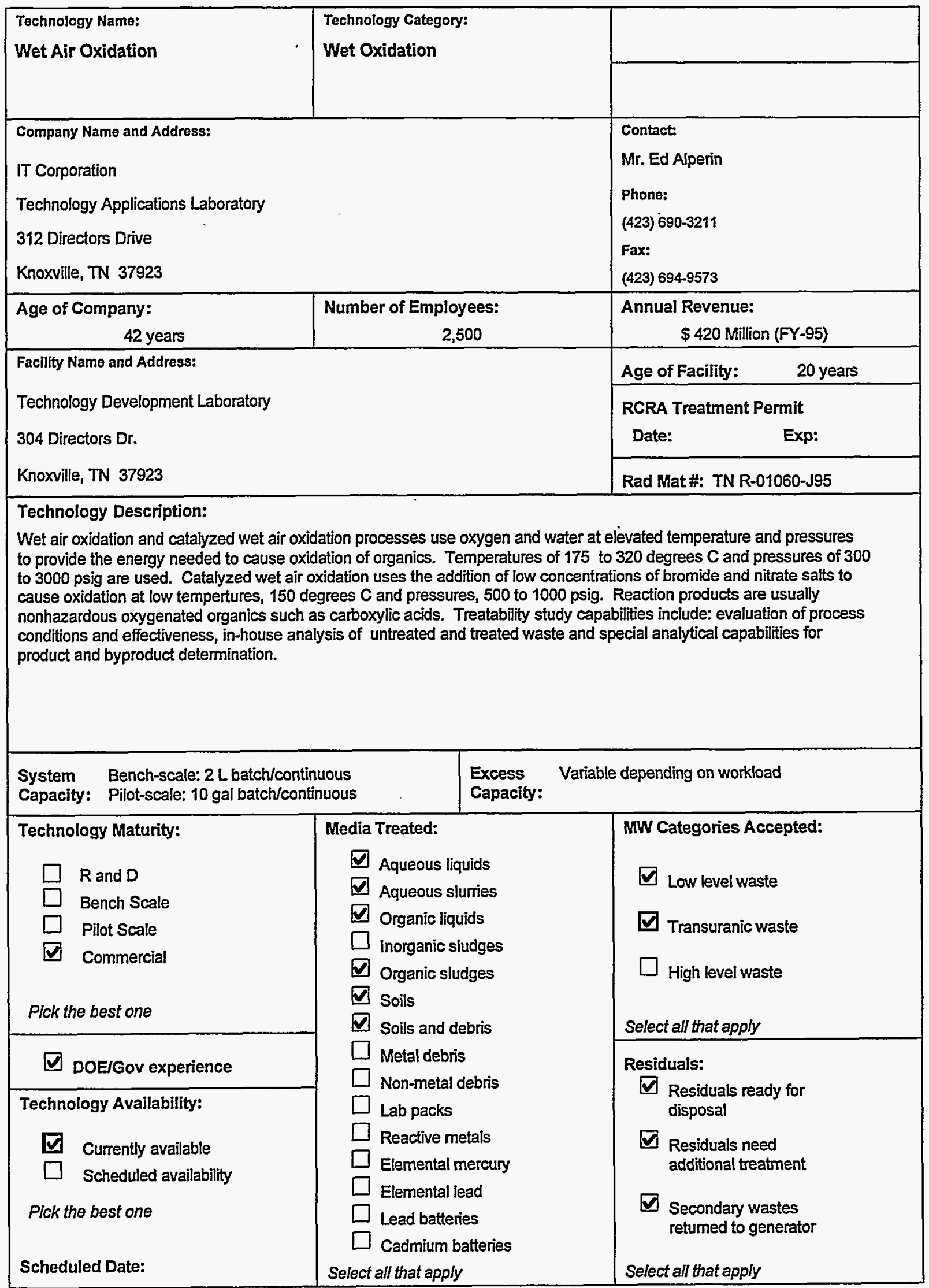


MIXED WASTE TREATABILITY STUDY TECHNOLOGY QUESTIONAIRE

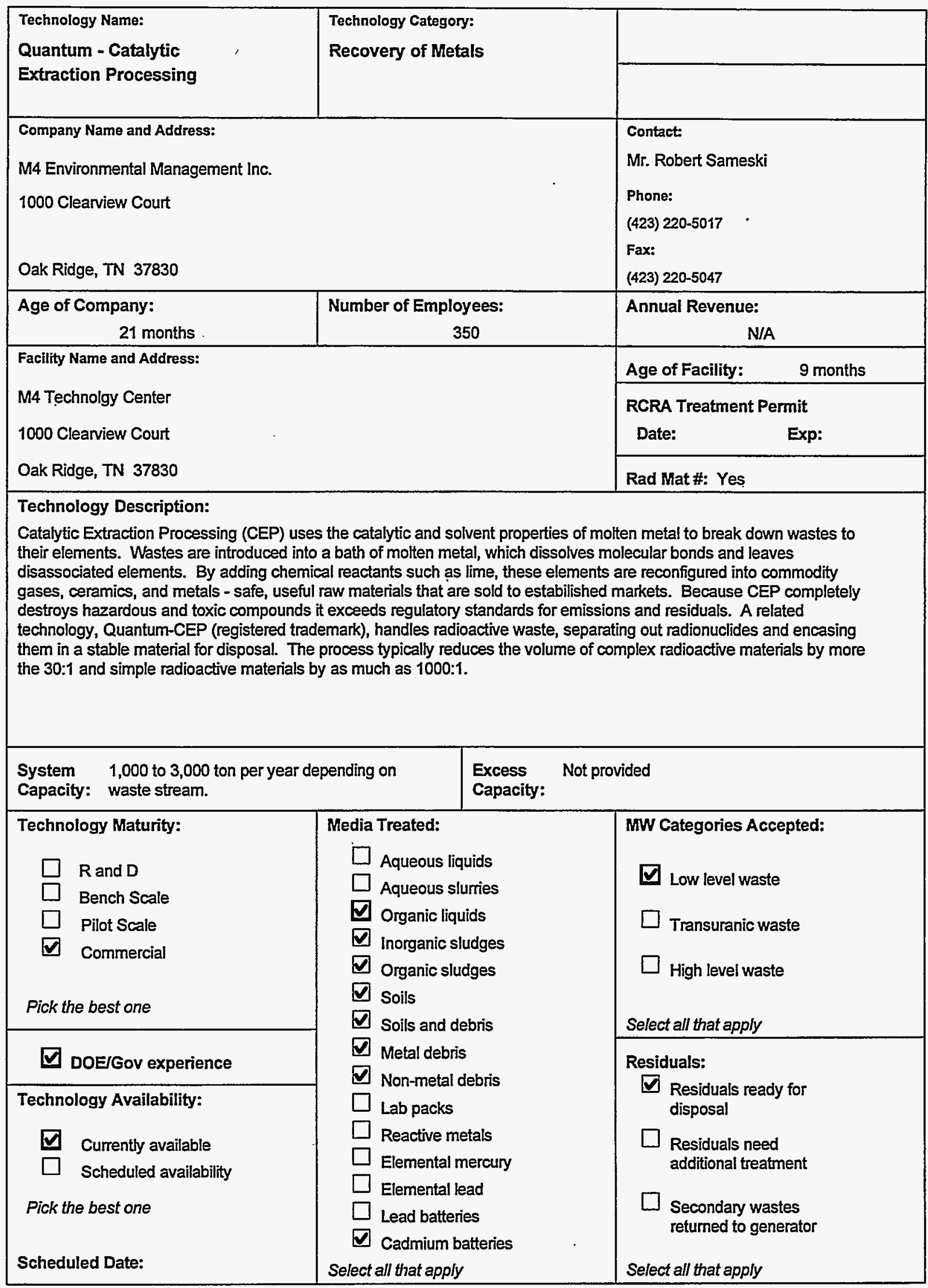


MIXED WASTE TREATABILITY STUDY TECHNOLOGY QUESTIONAIRE

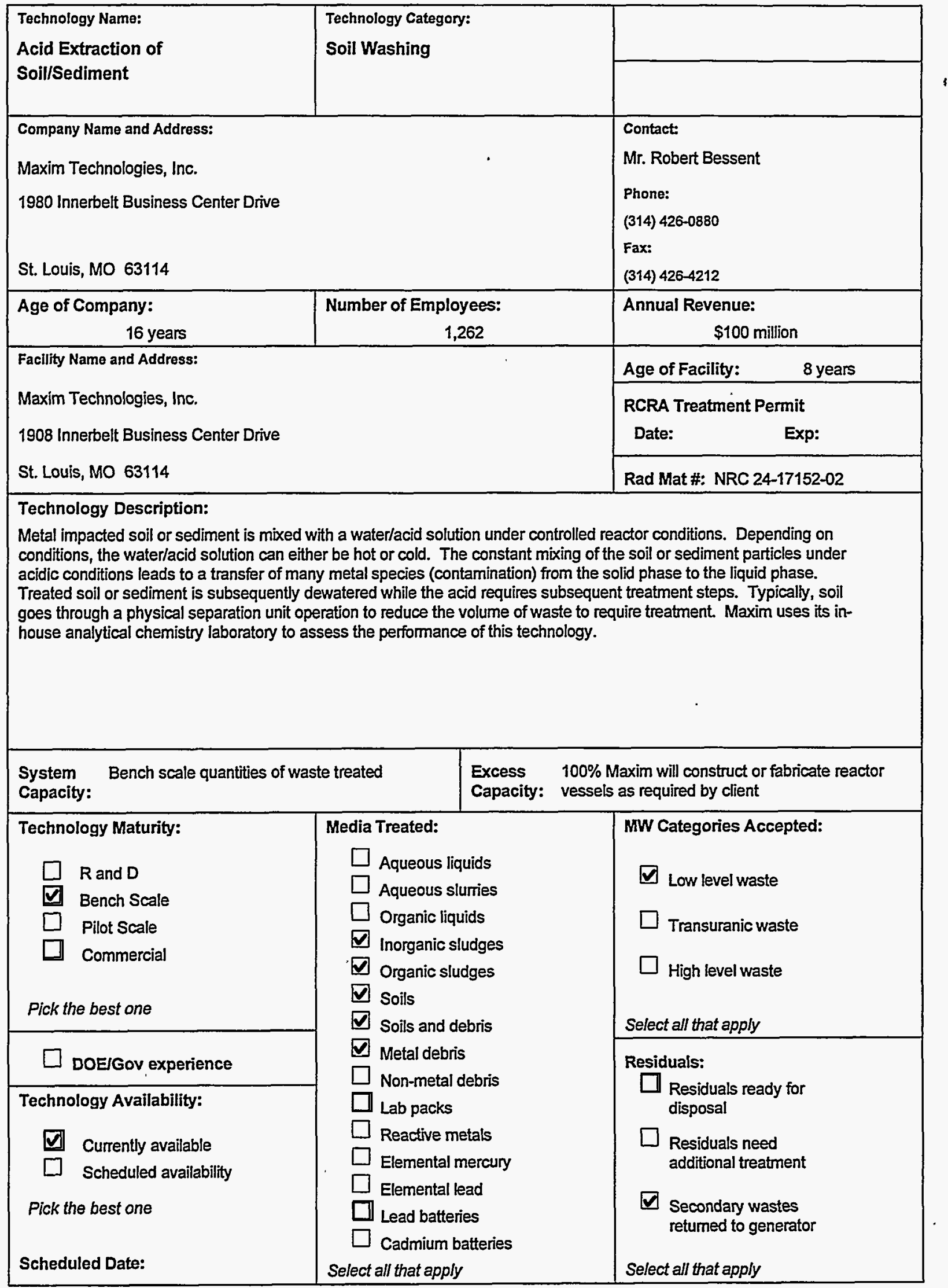


MIXED WASTE TREATABILITY STUDY TECHNOLOGY QUESTIONAIRE

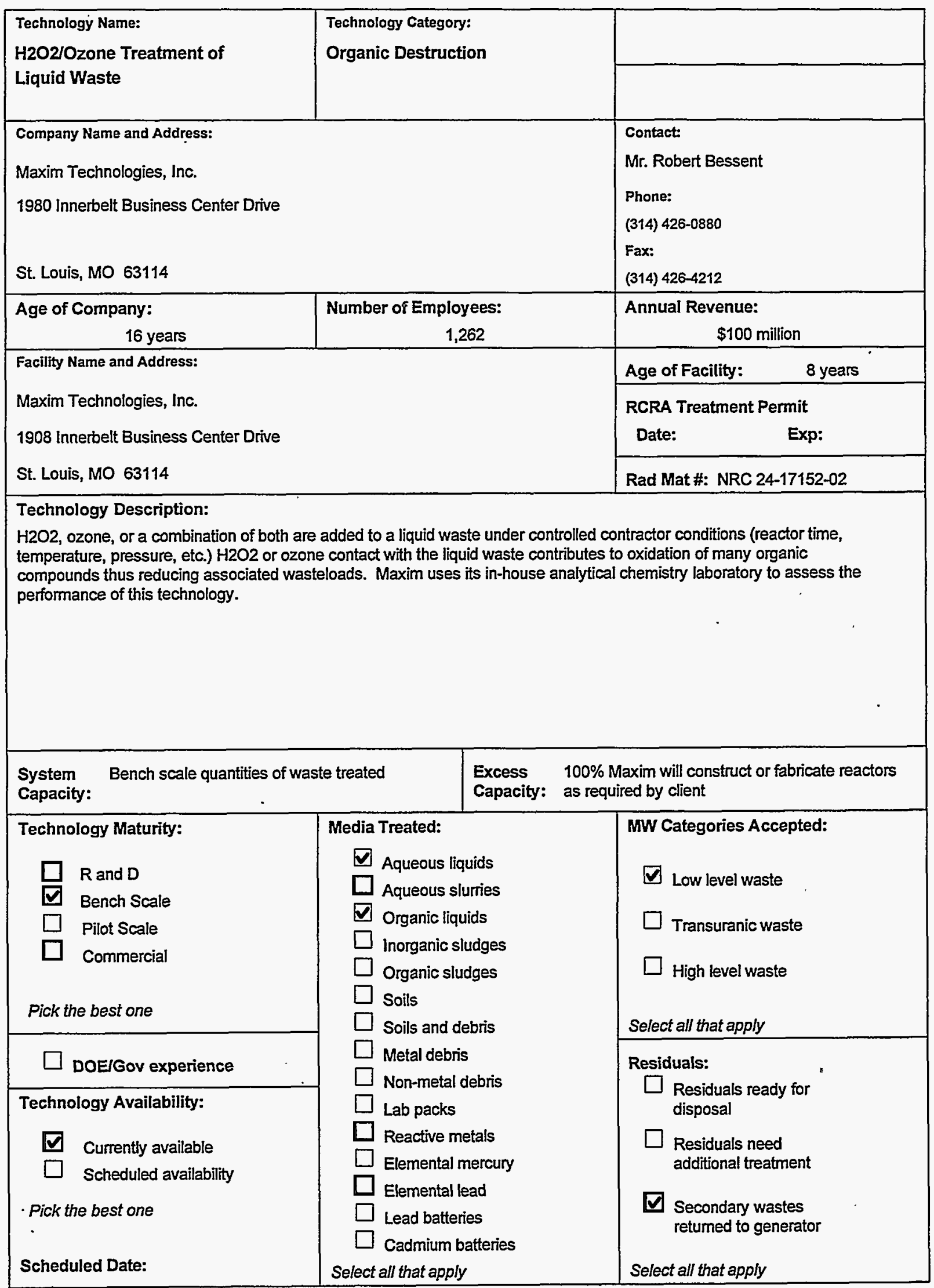


MIXED WASTE TREATABILITY STUDY TECHNOLOGY QUESTIONAIRE

\begin{tabular}{|c|c|c|c|}
\hline $\begin{array}{l}\text { Technology Name: } \\
\text { Modified Activated Sludge }\end{array}$ & \multicolumn{2}{|c|}{$\begin{array}{l}\text { Technology Category: } \\
\text { Biodegradation }\end{array}$} & \\
\hline \multicolumn{3}{|l|}{$\begin{array}{l}\text { Company Namo and Address: } \\
\text { Maxim Technologies, Inc. } \\
1980 \text { Innerbelt Business Center Drive }\end{array}$} & $\begin{array}{l}\text { Contact: } \\
\text { Mr. Robert Bessent } \\
\text { Phone: } \\
\text { (314) } 426-0880 \\
\text { Fax: } \\
\text { (314) } 426-4212\end{array}$ \\
\hline $\begin{array}{r}\text { Age of Company: } \\
16 \text { years }\end{array}$ & \multicolumn{2}{|c|}{$\begin{array}{l}\text { Number of Employees: } \\
\qquad 1,262\end{array}$} & $\begin{array}{l}\text { Annual Revenue: } \\
\qquad \$ 100 \text { million } \\
\end{array}$ \\
\hline \multirow{3}{*}{\multicolumn{3}{|c|}{$\begin{array}{l}\text { Facility Name and Address: } \\
\text { Maxim Technologies, Inc. } \\
1908 \text { Innerbelt Business Center Drive } \\
\text { St. Louis, MO } 63114\end{array}$}} & Age of Facility: \\
\hline & & & $\begin{array}{l}\text { RCRA Treatment Permit } \\
\text { Date: } \quad \text { Exp: }\end{array}$ \\
\hline & & & Rad Mat \#: NRC 24-17152-02 \\
\hline \multicolumn{4}{|c|}{$\begin{array}{l}\text { Technology Description: } \\
\text { Impacted wastewater or groundwater is mixed, under aerobic conditions, with an acclimated population of microorganisms } \\
\text { in a controlled reactor environment. The microorganisms within the reactor then use the organic contaminants in the liquid } \\
\text { waste as substrate (carbon source). Maxim uses its in-house analytical chemistry laboratory to assess the performance of } \\
\text { this technolgy. }\end{array}$} \\
\hline \multicolumn{2}{|c|}{$\begin{array}{l}\text { System Bench scale quantites of waste treated } \\
\text { Capacity: }\end{array}$} & \multicolumn{2}{|c|}{$\begin{array}{l}\text { Excess } \quad 100 \% \text { Maxim will construct or fabricate } \\
\text { Capacity: bioreactors as required by client }\end{array}$} \\
\hline $\begin{array}{c}\text { Technology Maturity: } \\
\square \text { R and D } \\
\square \quad \text { Bench Scale } \\
\square \quad \text { Pilot Scale } \\
\square \quad \text { Commercial } \\
\text { Pick the best one }\end{array}$ & \multirow{3}{*}{\multicolumn{2}{|c|}{$\begin{array}{l}\text { Media Treated: } \\
\qquad \text { Aqueous liquids } \\
\square \text { Aqueous slurries } \\
\square \text { Organic liquids } \\
\square \text { Inorganic sludges } \\
\square \text { Organic sludges } \\
\square \text { Soils } \\
\square \text { Soils and debris } \\
\square \text { Metal debris } \\
\square \text { Non-metal debris } \\
\square \text { Lab packs } \\
\square \text { Reactive metals } \\
\square \text { Elemental mercury } \\
\square \text { Elemental lead } \\
\square \text { Lead batteries } \\
\square \text { Cadmium batteries } \\
\text { Select all that apply }\end{array}$}} & $\begin{array}{l}\text { MW Categories Accepted: } \\
\square \text { Low level waste } \\
\square \text { Transuranic waste } \\
\square \text { High level waste } \\
\text { Select all that apply }\end{array}$ \\
\hline$\square$ DOE/Gov experience & & & Residuals: \\
\hline $\begin{array}{l}\text { Technology Availability: } \\
\qquad \begin{array}{l}\square \\
\square \text { Currently available }\end{array} \\
\text { Sicheduled availability } \\
\text { Scheduled Date: }\end{array}$ & & & $\begin{array}{l}\square \text { Residuals need } \\
\text { additional treatment } \\
\square \text { Secondary wastes } \\
\text { returned to generator }\end{array}$ \\
\hline
\end{tabular}




\section{MIXED WASTE TREATABILITY STUDY TECHNOLOGY QUESTIONAIRE}

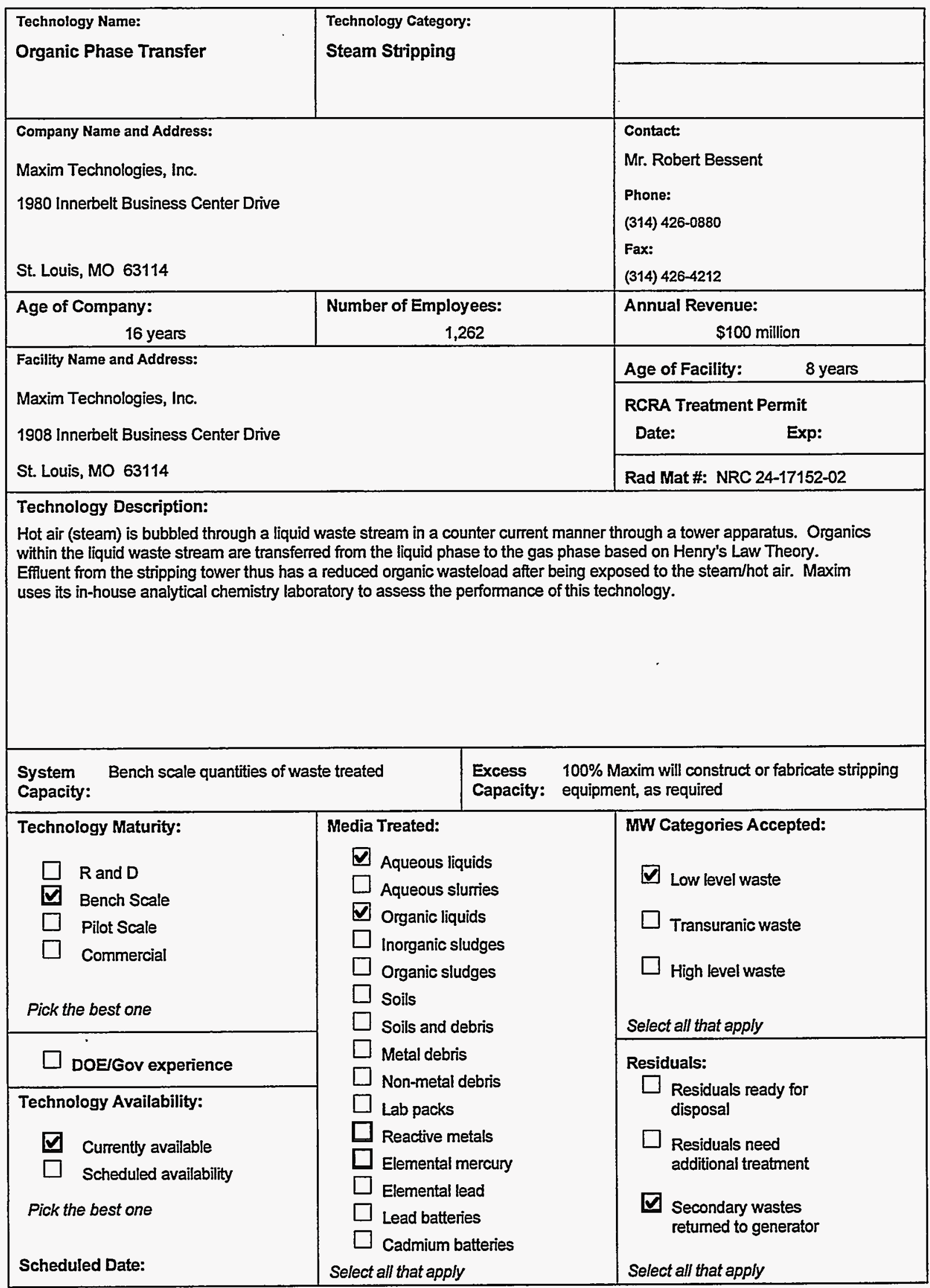


MIXED WASTE TREATABILITY STUDY TECHNOLOGY QUESTIONAIRE

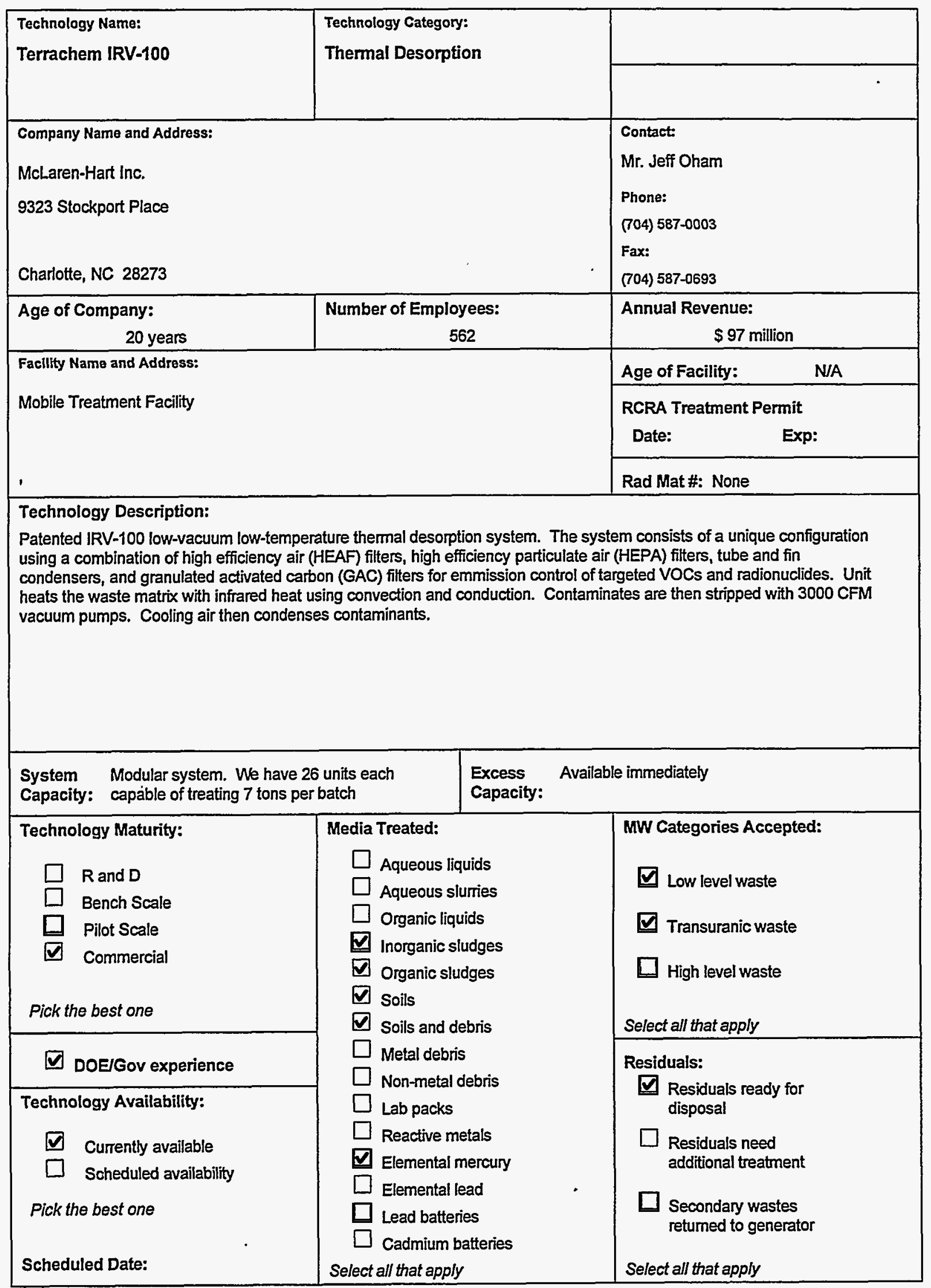


MIXED WASTE TREATABILITY STUDY TECHNOLOGY QUESTIONAIRE

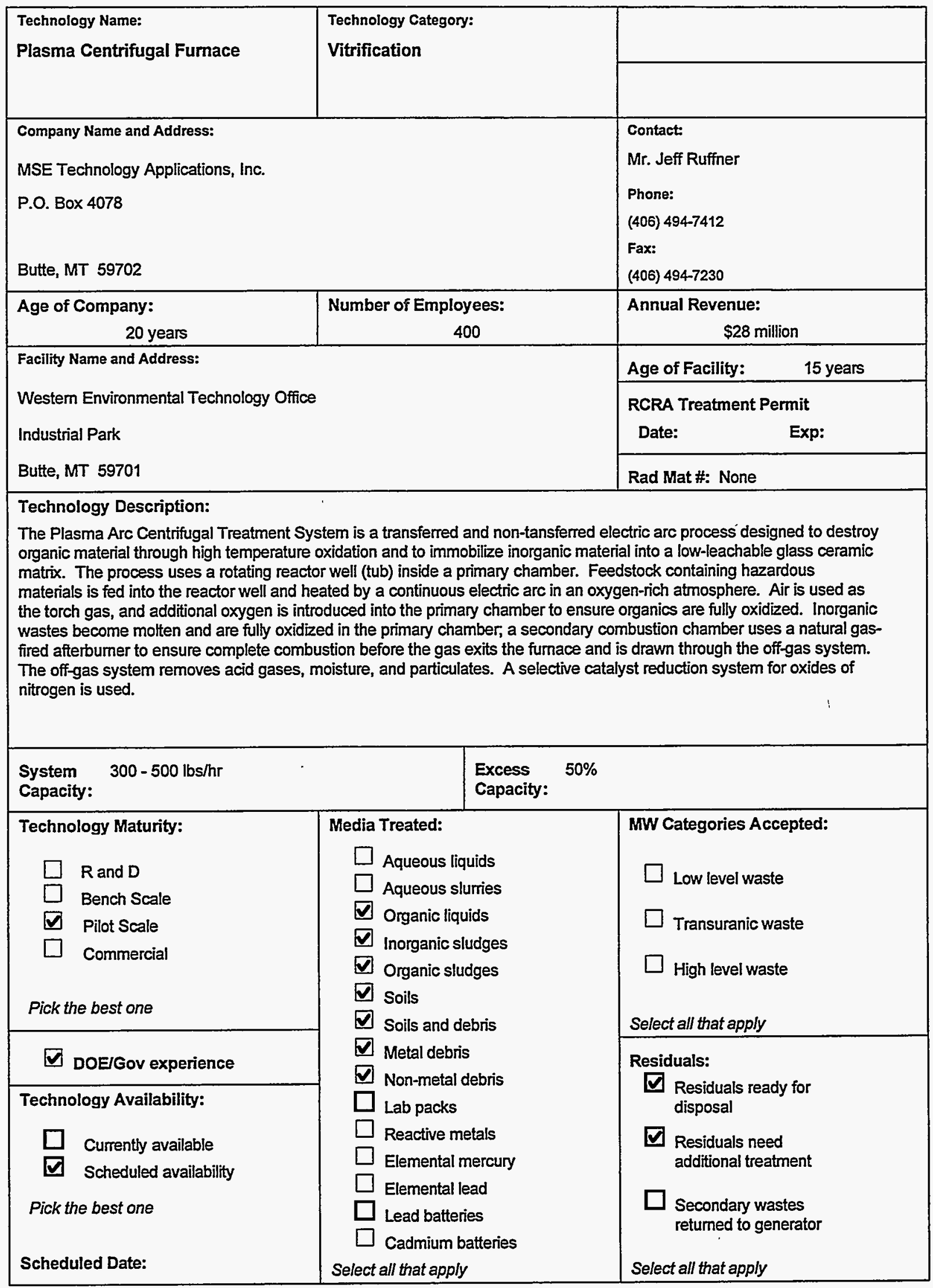




\section{MIXED WASTE TREATABILITY STUDY TECHNOLOGY QUESTIONAIRE}

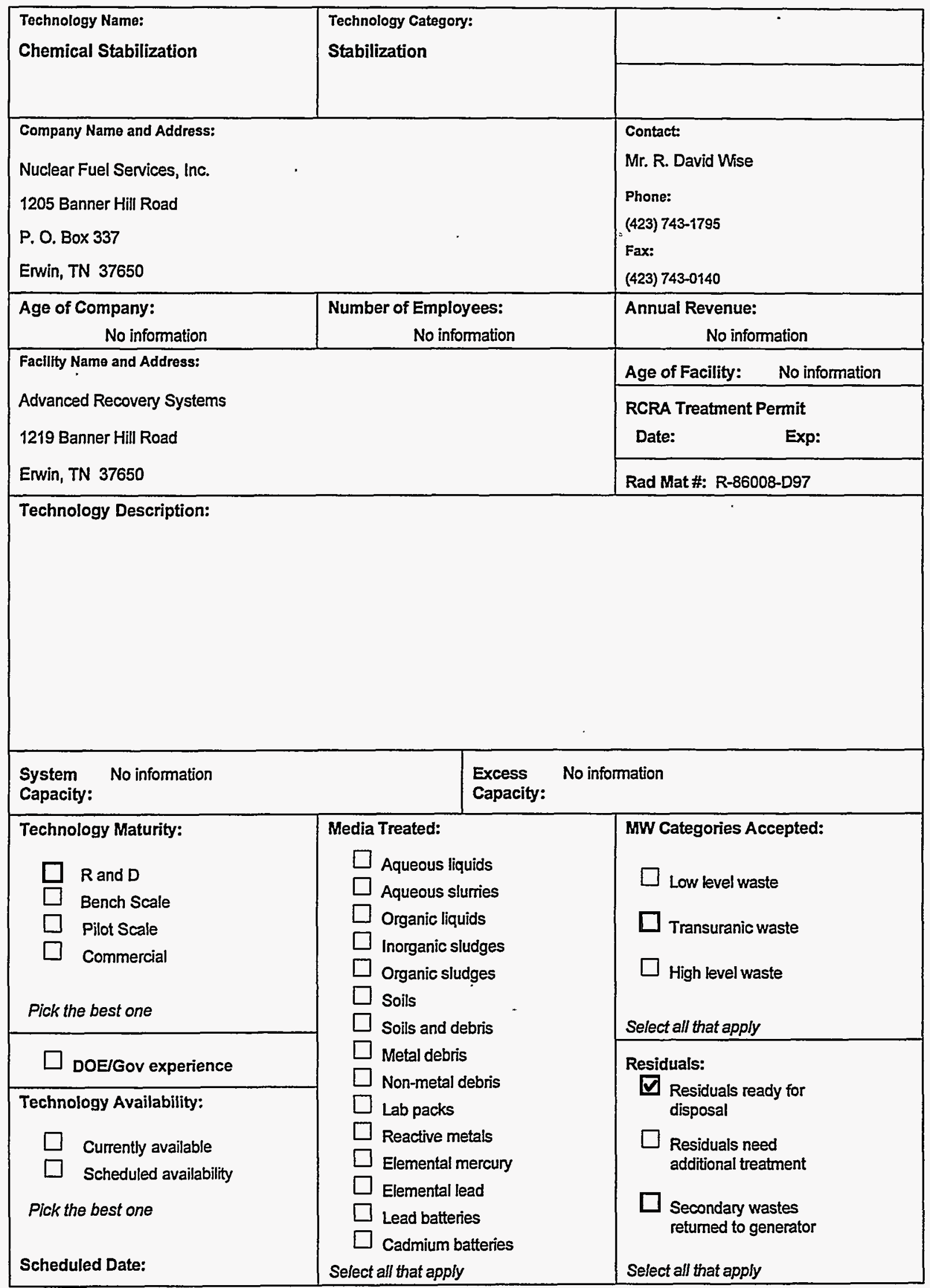


MIXED WASTE TREATABILITY STUDY TECHNOLOGY QUESTIONAIRE

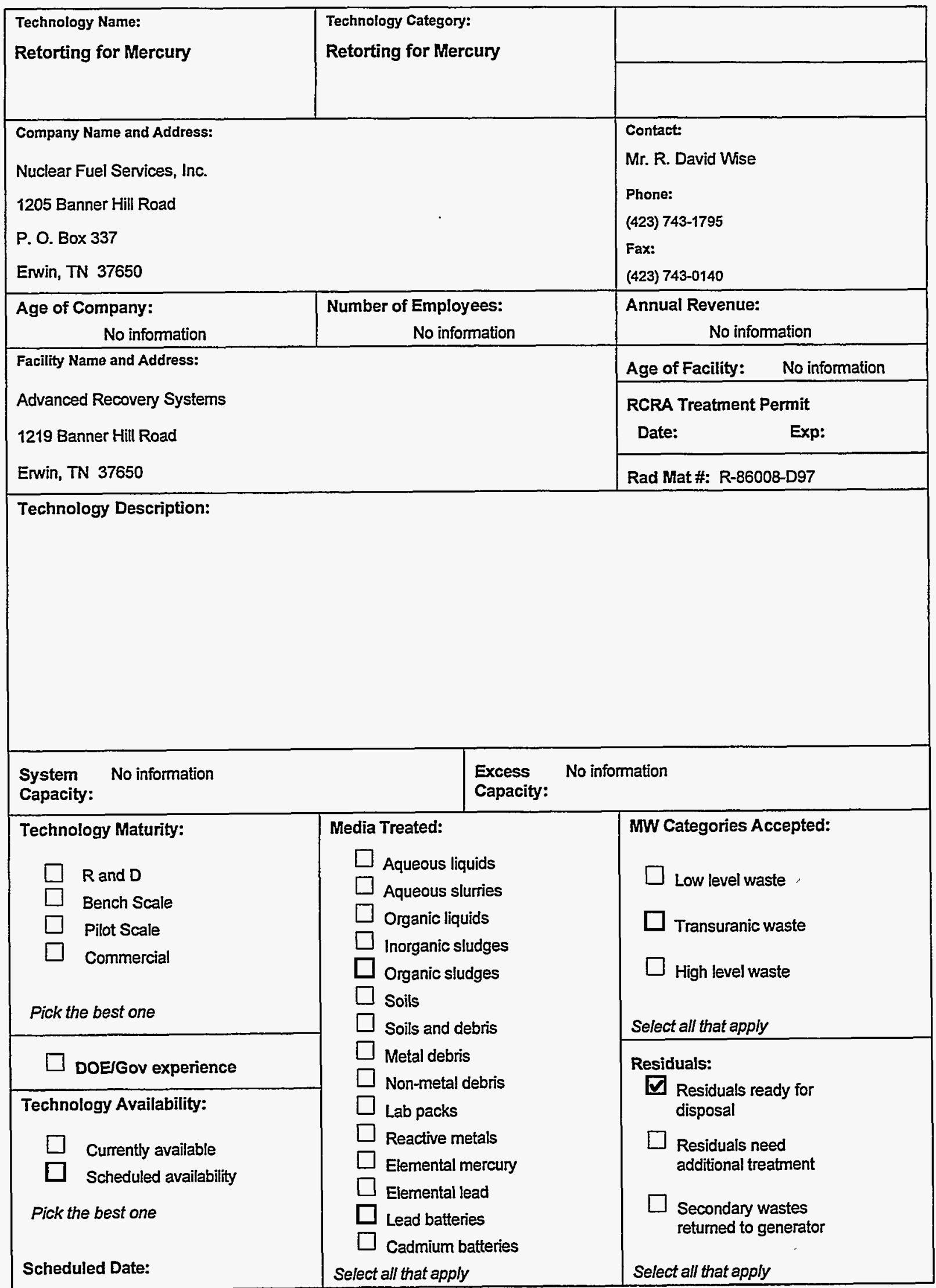


MIXED WASTE TREATABILITY STUDY TECHNOLOGY QUESTIONAIRE

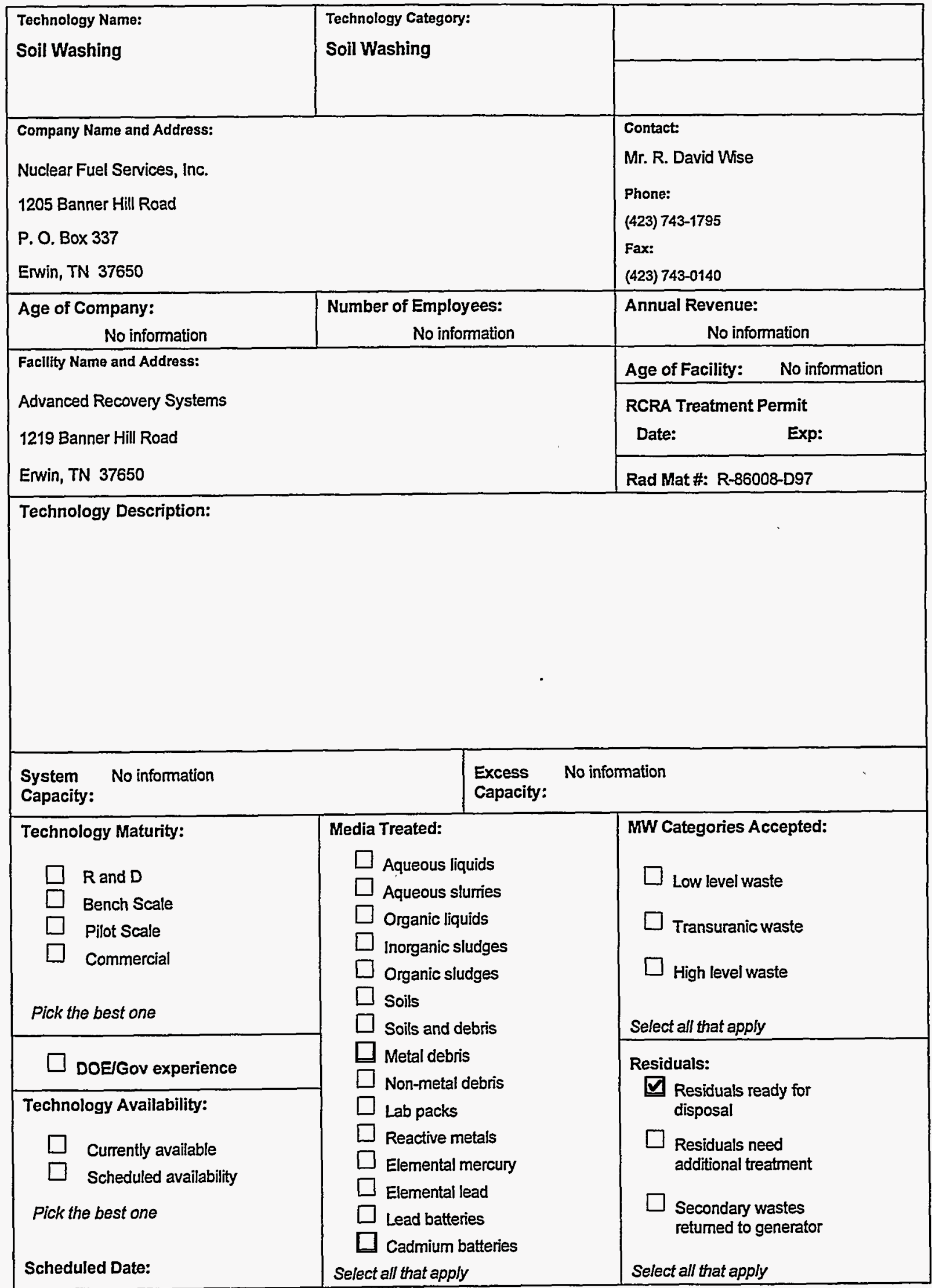




\section{MIXED WASTE TREATABILITY STUDY TECHNOLOGY QUESTIONAIRE}

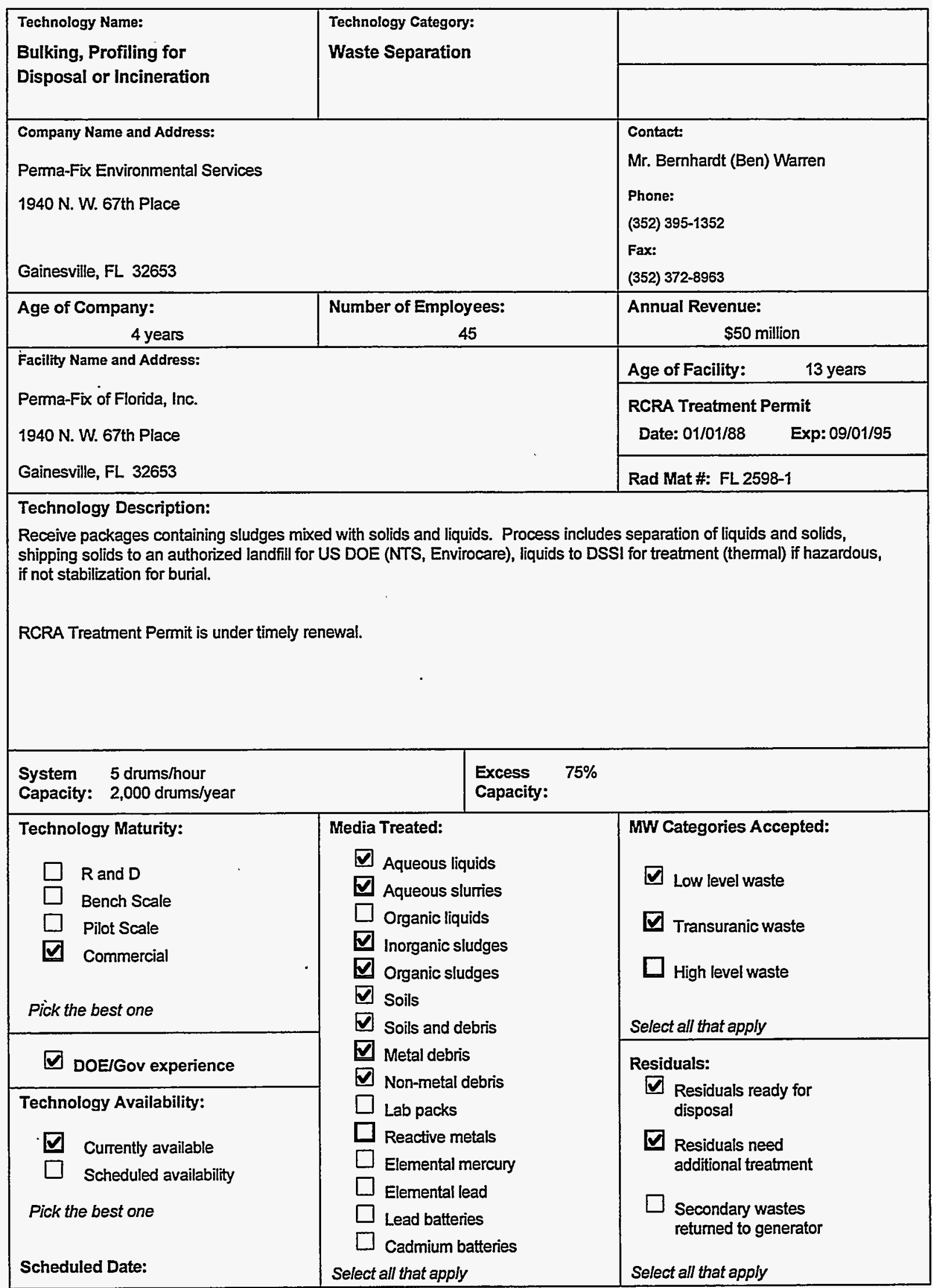


MIXED WASTE TREATABILITY STUDY TECHNOLOGY QUESTIONAIRE

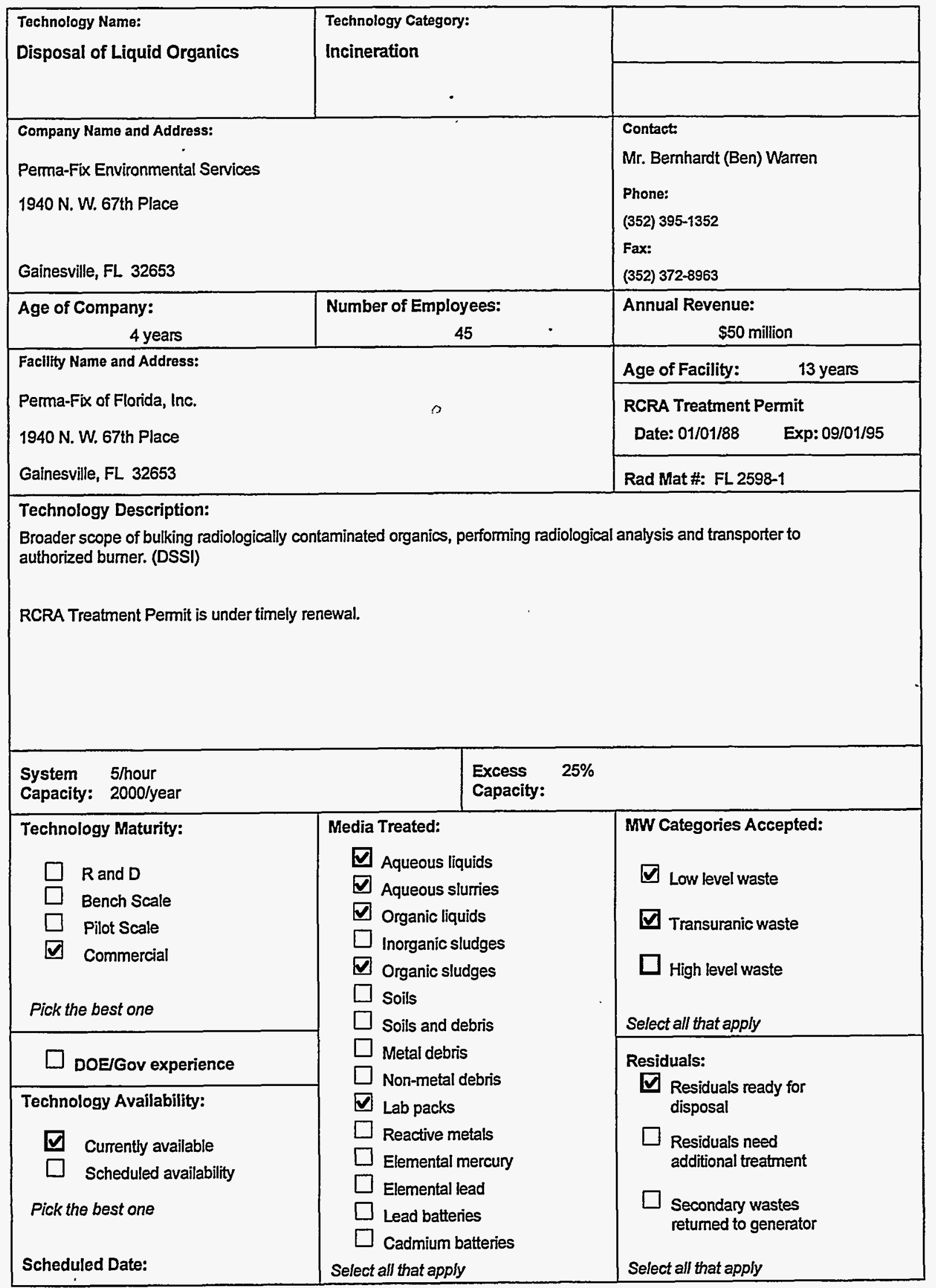


MIXED WASTE TREATABILITY STUDY TECHNOLOGY QUESTIONAIRE

\begin{tabular}{|c|c|c|}
\hline $\begin{array}{l}\text { Technology Name: } \\
\text { LSV Processing }\end{array}$ & $\begin{array}{l}\text { Technology Category: } \\
\text { Incineration }\end{array}$ & \\
\hline \multicolumn{2}{|l|}{$\begin{array}{l}\text { Company Name and Address: } \\
\text { Perma-Fix Environmental Services } \\
1940 \text { N. W. } 67 \text { th Place }\end{array}$} & $\begin{array}{l}\text { Contact: } \\
\text { Mr. Bernhardt (Ben) Warren } \\
\text { Phone: } \\
\text { (352) } 395-1352 \\
\text { Fax: } \\
(352) 372-8963\end{array}$ \\
\hline $\begin{array}{l}\text { Age of Company: } \\
\qquad 4 \text { years }\end{array}$ & $\begin{array}{c}\text { Number of Employees: } \\
45 \\
\end{array}$ & $\begin{array}{l}\text { Annual Revenue: } \\
\qquad 50 \text { million }\end{array}$ \\
\hline \multirow{3}{*}{\multicolumn{2}{|c|}{$\begin{array}{l}\text { Facility Name and Address: } \\
\text { Perma-Fix of Florida, Inc. } \\
1940 \text { N. W. } 67 \text { th Place } \\
\text { Gainesville, FL } 32653\end{array}$}} & Age of Facility: $\quad 13$ years \\
\hline & & $\begin{array}{l}\text { RCRA Treatment Permit } \\
\begin{array}{ll}\text { Date: } 01 / 01 / 88 & \text { Exp: 09/01/95 }\end{array}\end{array}$ \\
\hline & & Rad Mat \#: FL 2598-1 \\
\hline \multicolumn{3}{|l|}{$\begin{array}{l}\text { Technology Description: } \\
\text { Receipt and processing of lic } \\
\text { rinsate radiologically tested } \\
\text { bumed for heat recovery. }\end{array}$} \\
\hline \multicolumn{3}{|c|}{ RCRA Treatment Permit is under timely renewal. Previously, as Quadrex Environmental Co. Processing LSV since 1983.} \\
\hline $\begin{array}{ll}\text { System } & 10 \text { drums/hour } \\
\text { Capacity: } & 20,000 \text { drums/year }\end{array}$ & $\begin{array}{l}\text { Excess } \\
\text { Capacity: }\end{array}$ & \\
\hline $\begin{array}{l}\text { Technology Maturity: } \\
\square \text { R and D } \\
\square \quad \text { Bench Scale } \\
\square \text { Pilot Scale } \\
\square \quad \text { Commercial } \\
\text { Pick the best one }\end{array}$ & \multirow{3}{*}{$\begin{array}{l}\text { Media Treated: } \\
\square \text { Aqueous liquids } \\
\square \text { Aqueous slurries } \\
\square \text { Organic liquids } \\
\square \text { Inorganic sludges } \\
\square \text { Organic sludges } \\
\square \text { Soils } \\
\square \text { Soils and debris } \\
\square \text { Metal debris } \\
\square \text { Non-metal debris } \\
\square \text { Lab packs } \\
\square \text { Reactive metals } \\
\square \text { Elemental mercury } \\
\square \text { Elemental lead } \\
\square \text { Lead batteries } \\
\square \text { Cadmium batteries } \\
\text { Select all that apply }\end{array}$} & $\begin{array}{l}\text { MW Categories Accepted: } \\
\square \text { Low level waste } \\
\square \text { Transuranic waste } \\
\square \text { High level waste } \\
\text { Select all that apply }\end{array}$ \\
\hline$\square$ DOEGov experience & & Residuals: \\
\hline $\begin{array}{l}\text { Technology Availability: } \\
\qquad \begin{array}{l}\square \\
\square \text { Scheduled availability }\end{array} \\
\text { Pick the best one } \\
\text { Scheduled Date: }\end{array}$ & & $\begin{array}{l}\text { disposal } \\
\text { Residuals need } \\
\text { additional treatment } \\
\square \text { Secondary wastes } \\
\text { returned to generator } \\
\text { Select all that apply }\end{array}$ \\
\hline
\end{tabular}




\section{MIXED WASTE TREATABILITY STUDY TECHNOLOGY QUESTIONAIRE}

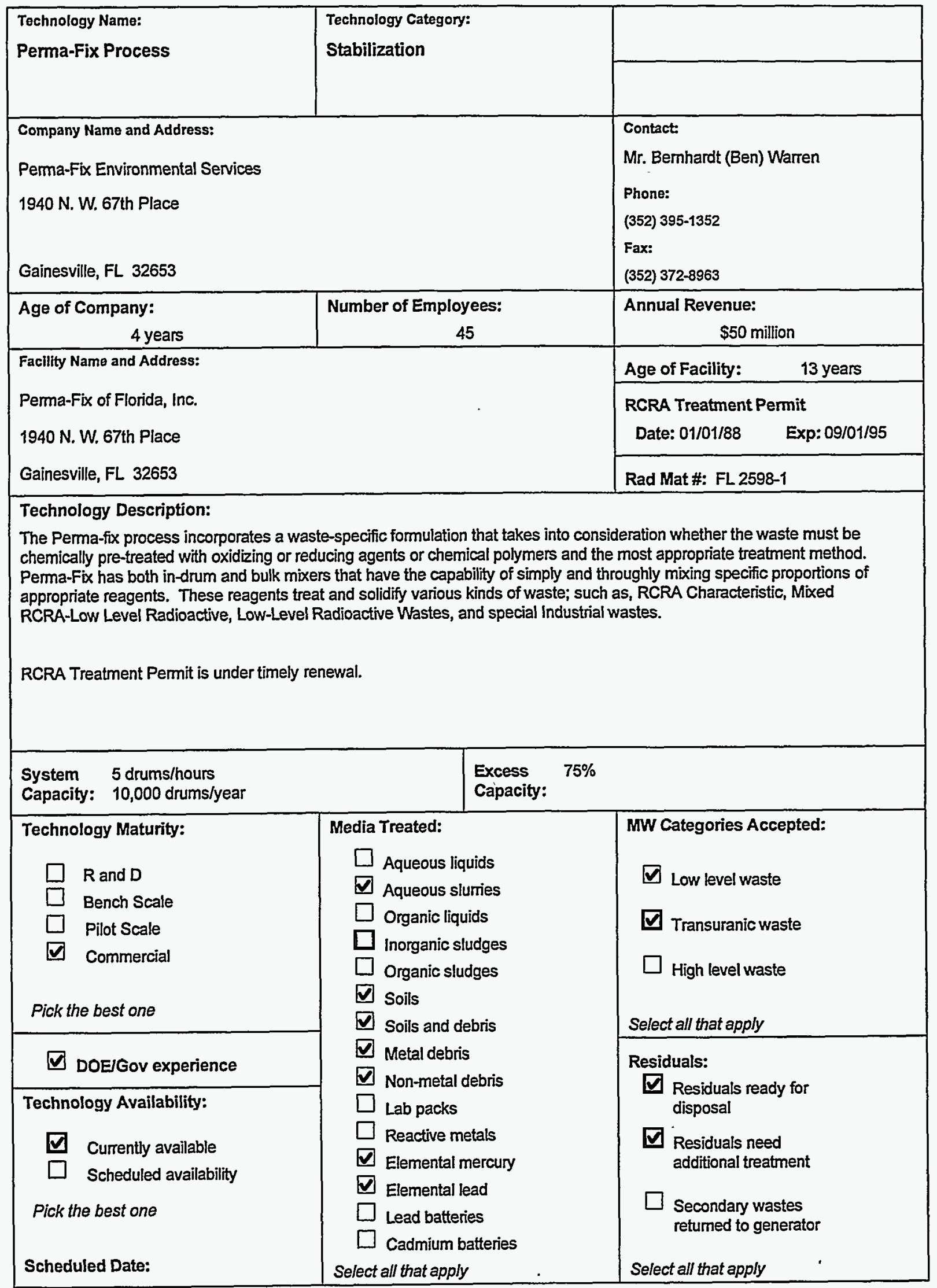


MIXED WASTE TREATABILITY STUDY TECHNOLOGY QUESTIONAIRE

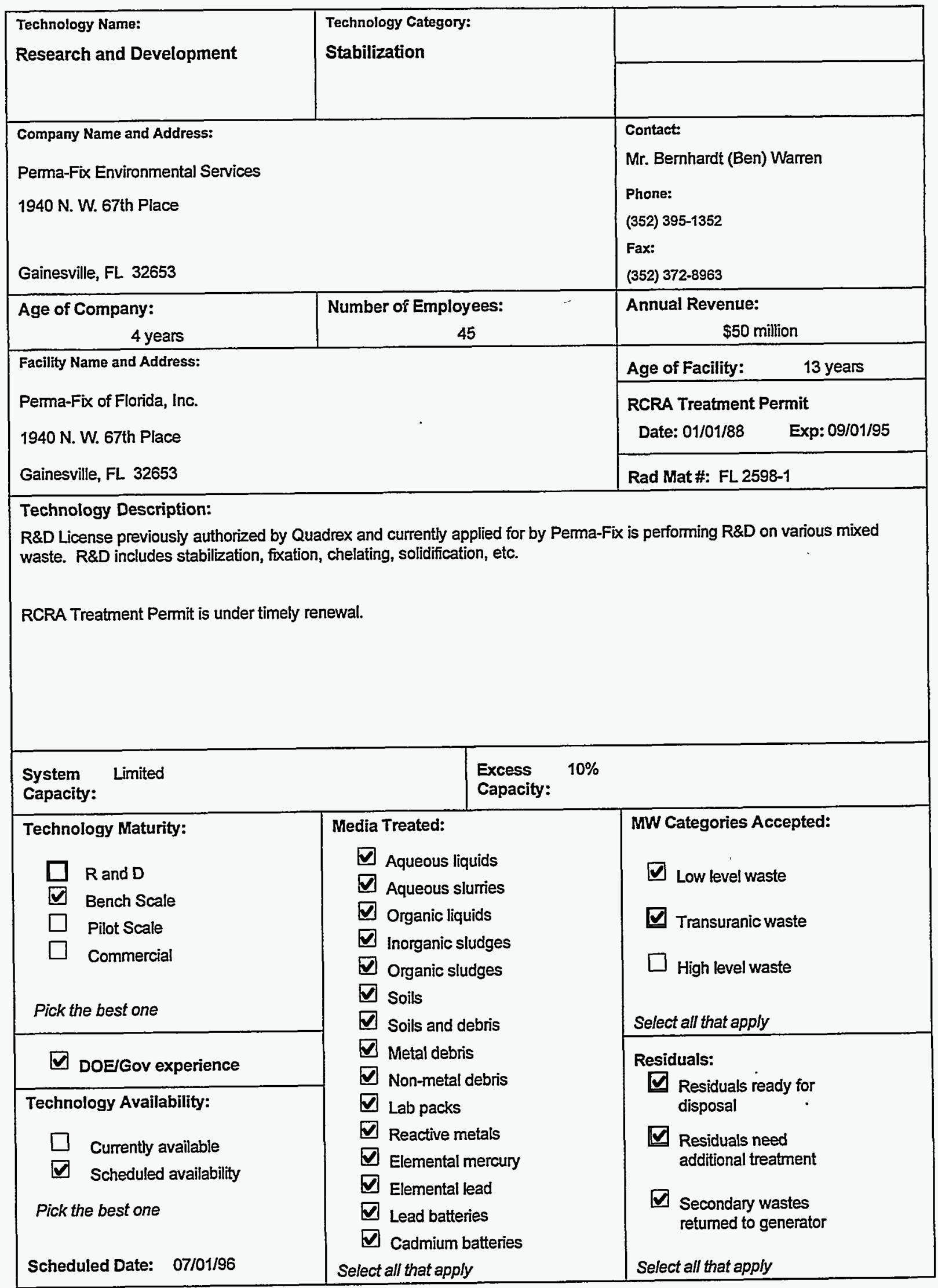


MIXED WASTE TREATABILITY STUDY TECHNOLOGY QUESTIONAIRE

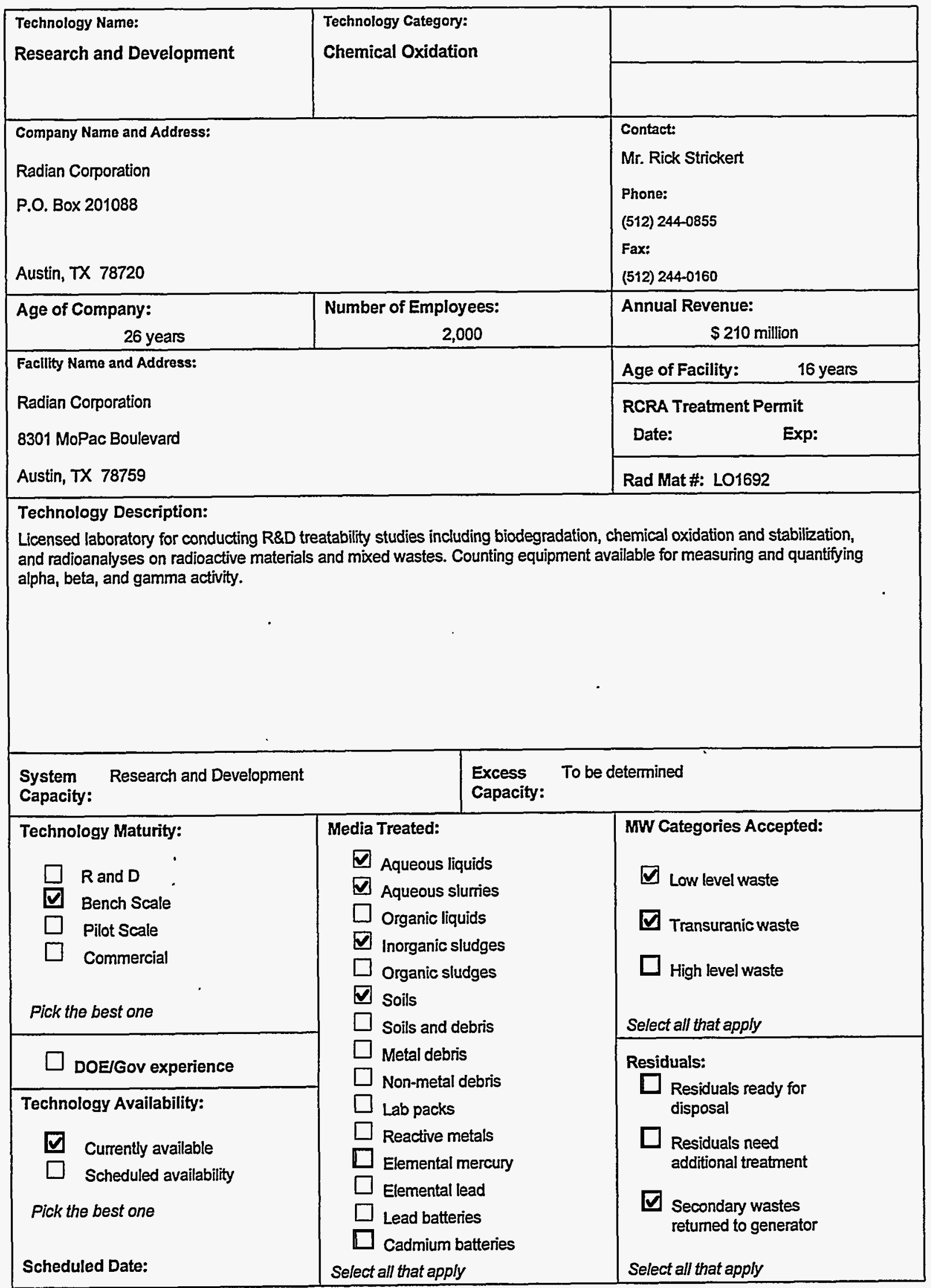


MIXED WASTE TREATABILITY STUDY TECHNOLOGY QUESTIONAIRE

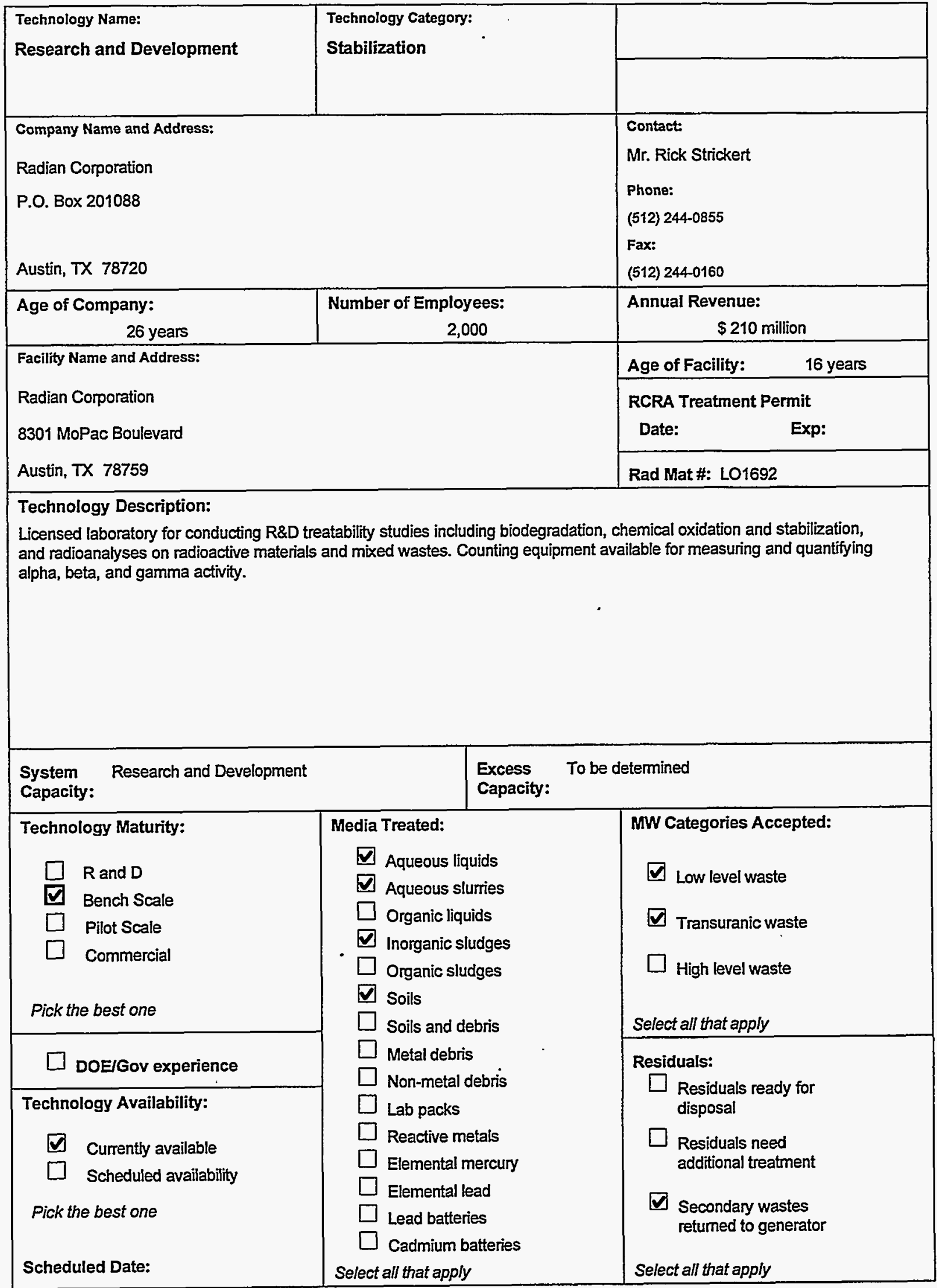


MIXED WASTE TREATABILITY STUDY TECHNOLOGY QUESTIONAIRE

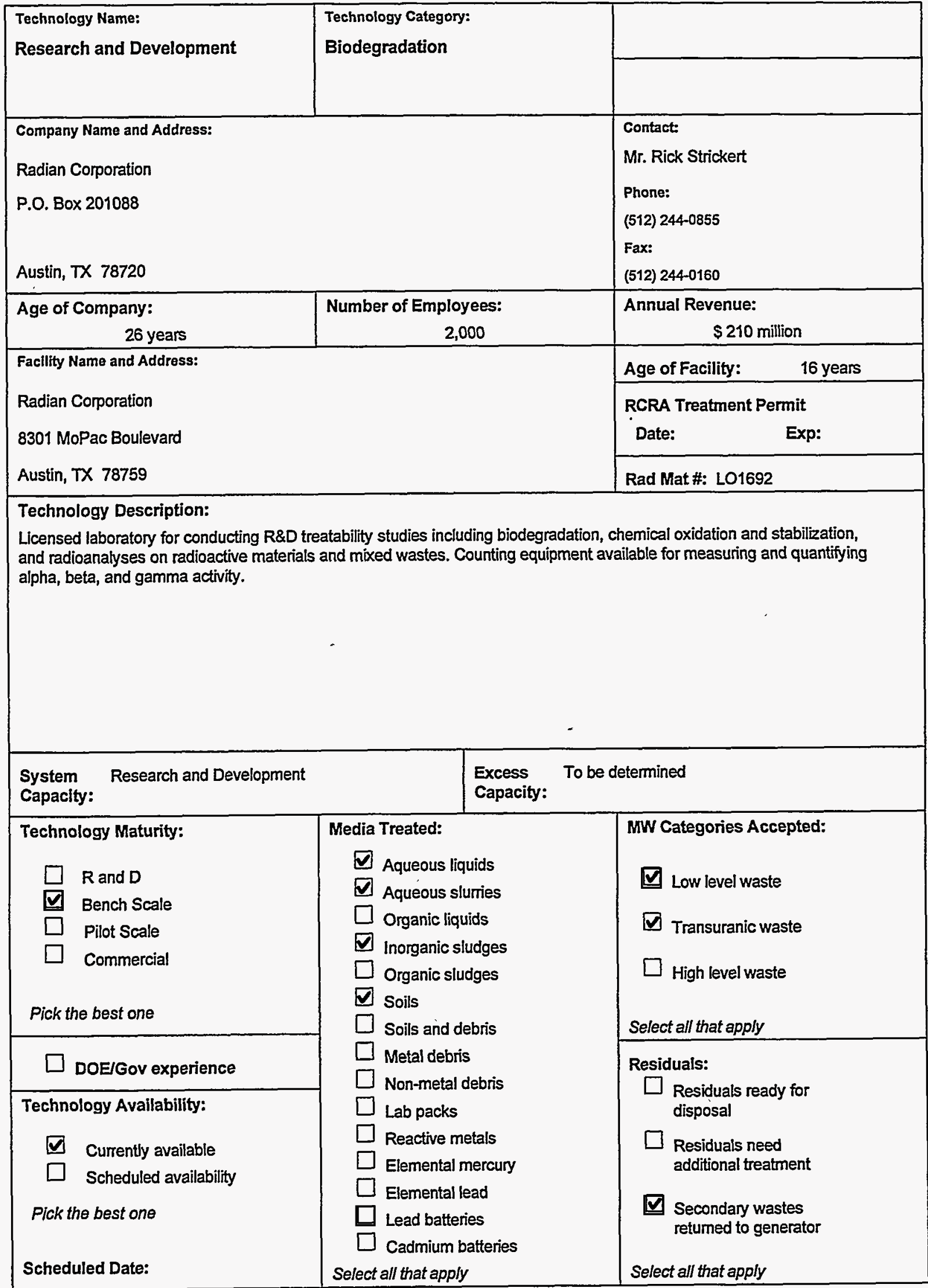


MIXED WASTE TREATABILITY STUDY TECHNOLOGY QUESTIONAIRE

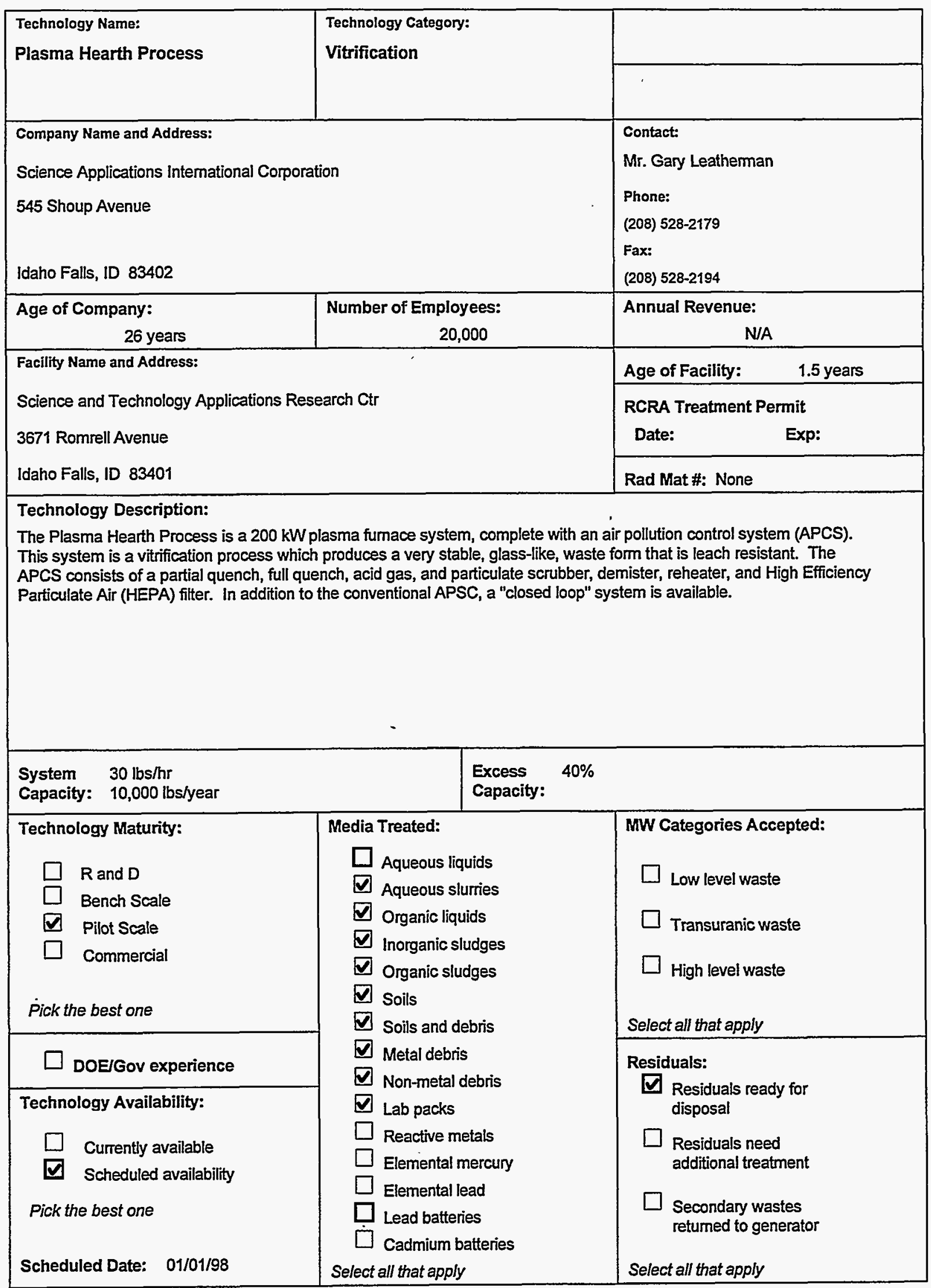


MIXED WASTE TREATABILITY STUDY TECHNOLOGY QUESTIONAIRE

\begin{tabular}{|c|c|c|}
\hline $\begin{array}{l}\text { Technology Name: } \\
\text { Cement Stabilization }\end{array}$ & $\begin{array}{l}\text { Technology Category: } \\
\text { Stabilization }\end{array}$ & \\
\hline \multicolumn{2}{|l|}{$\begin{array}{l}\text { Company Name and Address: } \\
\text { Scientific Ecology Group, Inc. } \\
\text { P.O. Box } 2530 \\
1560 \text { Bear Creek Road } \\
\text { Oak Ridge, TN } 37830\end{array}$} & $\begin{array}{l}\text { Contact: } \\
\text { Mr. Marty Brownstein } \\
\text { Phone: } \\
\text { (423) } 376-8321 \\
\text { Fax: }\end{array}$ \\
\hline $\begin{array}{r}\text { Age of Company: } \\
10 \text { years }\end{array}$ & $\begin{array}{r}\text { Number of Employees: } \\
1300 \\
\end{array}$ & $\begin{array}{r}\text { Annual Revenue: } \\
\$ 150 \mathrm{M} \\
\end{array}$ \\
\hline \multirow{3}{*}{\multicolumn{2}{|c|}{$\begin{array}{l}\text { Facility Name and Address: } \\
\text { Scientific Ecology Group, Inc. } \\
1560 \text { Bear Creek Rd. } \\
\text { Oak Ridge, TN } 37830\end{array}$}} & Age of Facility: \\
\hline & & $\begin{array}{l}\text { RCRA Treatment Permit } \\
\text { Date: } \quad \text { Exp: }\end{array}$ \\
\hline & & Rad Mat \#: R-73016-F96 \\
\hline \multicolumn{3}{|c|}{$\begin{array}{l}\text { Technology Description: } \\
\text { Cement solidification is a key tool for remediating hazardous wastes. The technique consists of entrapping the wastes } \\
\text { within a solid matrix having high structural integrity, which minimizes the risk of escape by leaching. The cement } \\
\text { stabilization process involves several steps. First the waste is pretreated to remove the non-contaminated debris. Second } \\
\text { the volume is reduced to } 40-50 \% \text { solids. The solids are batched and pH adjusted, then fed into the processor for mixing } \\
\text { with cement binder. }\end{array}$} \\
\hline $\begin{array}{ll}\text { System } & 500 \mathrm{lbs} / \mathrm{hr} \\
\text { Capacity: } & 110,000 \mathrm{lbs} / \text { annually }\end{array}$ & $\begin{array}{l}\text { Excess } \quad 80 \% \\
\text { Capacity: }\end{array}$ & \\
\hline $\begin{array}{c}\text { Technology Maturity: } \\
\square \text { R and D } \\
\square \quad \text { Bench Scale } \\
\square \quad \text { Pilot Scale } \\
\square \quad \text { Commercial } \\
\text { Pick the best one }\end{array}$ & \multirow{4}{*}{$\begin{array}{l}\text { Media Treated: } \\
\square \text { Aqueous liquids } \\
\square \text { Aqueous slurries } \\
\square \text { Organic liquids } \\
\square \text { Inorganic sludges } \\
\square \text { Organic sludges } \\
\square \text { Soils } \\
\square \text { Soils and debris } \\
\square \text { Metal debris } \\
\square \text { Non-metal debris } \\
\square \text { Lab packs } \\
\square \text { Reactive metals } \\
\square \text { Elemental mercury } \\
\square \text { Elemental lead } \\
\square \text { Lead batteries } \\
\square \text { Cadmium batteries } \\
\text { Select all that apply }\end{array}$} & $\begin{array}{l}\text { MW Categories Accepted: } \\
\square \text { Low level waste } \\
\square \text { Transuranic waste } \\
\square \text { High level waste } \\
\text { Select all that apply }\end{array}$ \\
\hline$\square$ DOEJGov experience & & $\begin{array}{l}\text { Residuals: } \\
\text { Residuals ready for }\end{array}$ \\
\hline Technology Availability: & & disposal \\
\hline $\begin{array}{l}\square \text { Currently available } \\
\square \text { Scheduled availability } \\
\text { Pick the best one } \\
\text { Scheduled Date: }\end{array}$ & & $\begin{array}{l}\square \text { Residuals need } \\
\text { additional treatment } \\
\square \text { Secondary wastes } \\
\text { returned to generator } \\
\text { Select all that apply }\end{array}$ \\
\hline
\end{tabular}


MIXED WASTE TREATABILITY STUDY TECHNOLOGY QUESTIONAIRE

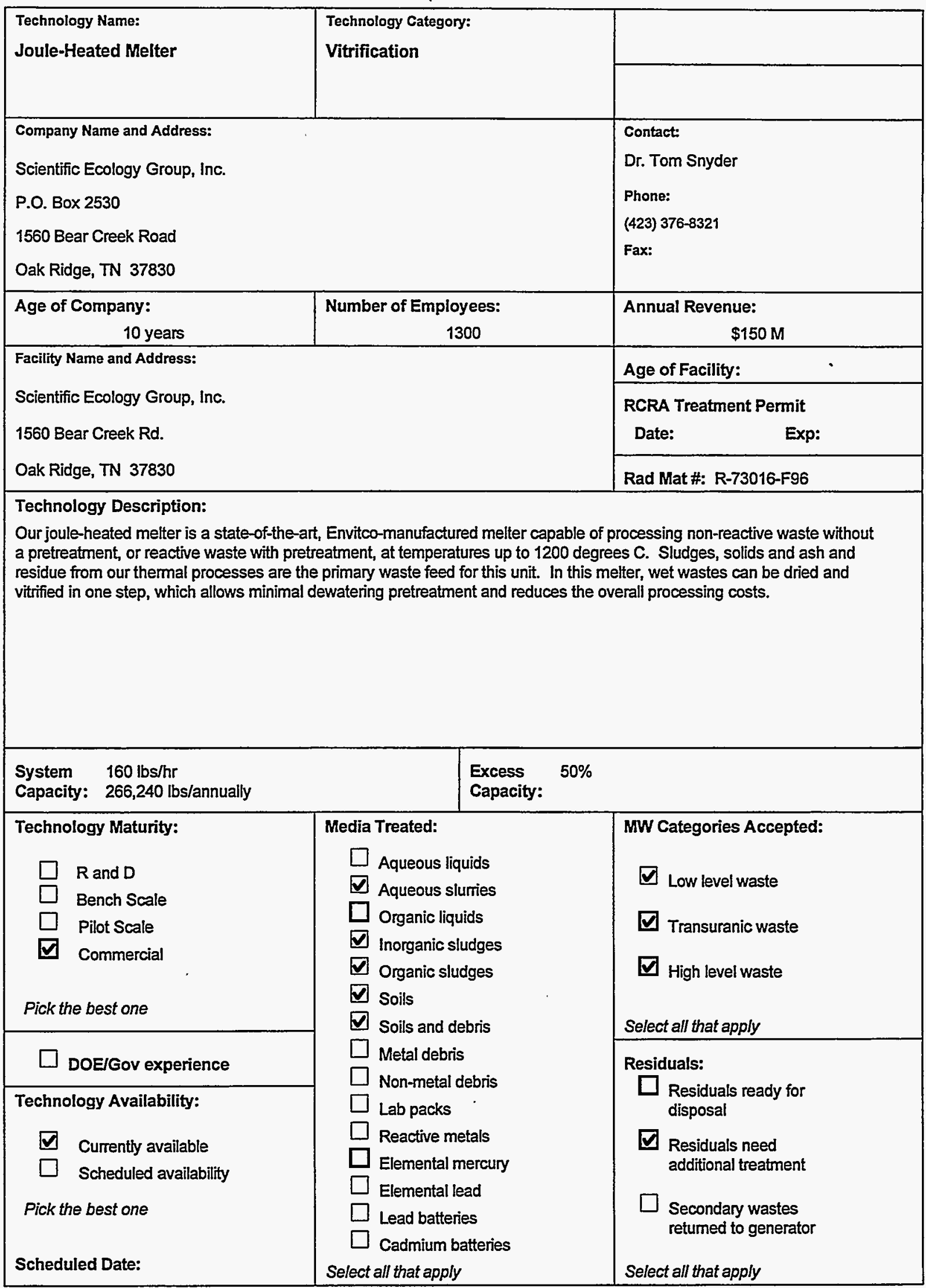


MIXED WASTE TREATABILITY STUDY TECHNOLOGY QUESTIONAIRE

\begin{tabular}{|c|c|c|}
\hline $\begin{array}{l}\text { Technology Name: } \\
\text { Magnesium Oxide Cement } \\
\text { Stabilization }\end{array}$ & $\begin{array}{l}\text { Technology Category: } \\
\text { Stabilization }\end{array}$ & \\
\hline \multicolumn{2}{|l|}{$\begin{array}{l}\text { Company Name and Address: } \\
\text { Scientific Ecology Group, Inc. } \\
\text { P.O. Box } 2530 \\
1560 \text { Bear Creek Road } \\
\text { Oak Ridge, TN } 37830\end{array}$} & $\begin{array}{l}\text { Contact: } \\
\text { Mr. Marty Brownstein } \\
\text { Phone: } \\
\text { (423) } 376-8321 \\
\text { Fax: }\end{array}$ \\
\hline $\begin{array}{l}\text { Age of Company: } \\
10 \text { years }\end{array}$ & $\begin{array}{r}\text { Number of Employees: } \\
1300 \\
\end{array}$ & $\begin{array}{r}\text { Annual Revenue: } \\
\$ 150 \mathrm{M}\end{array}$ \\
\hline \multirow{3}{*}{\multicolumn{2}{|c|}{$\begin{array}{l}\text { Facillty Name and Address: } \\
\text { Scientific Ecology Group, Inc. } \\
1560 \text { Bear Creek Rd. } \\
\text { Oak Ridge, TN } 37830 \\
\end{array}$}} & Age of Facility: \\
\hline & & $\begin{array}{l}\text { RCंRA Treatment Permit } \\
\text { Date: }\end{array}$ \\
\hline & & Rad Mat \#: R-73016-F96 \\
\hline \multicolumn{3}{|c|}{$\begin{array}{l}\text { Technology Description: } \\
\text { SEG currently uses magnesium oxide (Oxymag) as the media of choice to solidify mixed wastes generated during SEG } \\
\text { porcess operations. Oxymag effectively stabilizes waste types like incinerator ash, mixed waste fly ash, baghouse dust, } \\
\text { and furnace waste that have heretofore defied solidification and stabilization in concrete in any significant quanitity. } \\
\text { Oxymag cement is blended with a mixture of waste, potable or contaminated water, and magnesium sulfate in a rotary } \\
\text { mixer at ambient temperature and pressure. The resultant concrete produced is pH adjusted to } 9.75 \text { by the magnesium } \\
\text { oxide. The result is a cement that provides the desired stabilization of the waste form and provides a pH level that } \\
\text { optimizes the insoulubility of any heavy metals bound in the matrix. The stable pH of the Oxymag cement allows waste } \\
\text { loadings of } 50 \text { - } 60 \text { wt\% waste, thus minimizing the disposal volume of the stabilized waste form. }\end{array}$} \\
\hline $\begin{array}{ll}\text { System } & 500 \mathrm{lbs} / \mathrm{hr} \\
\text { Capacity: } & 110,000 \mathrm{lbs} / \text { annually }\end{array}$ & $\begin{array}{l}\text { Excess } \quad 80 \% \\
\text { Capacity: }\end{array}$ & \\
\hline $\begin{array}{c}\text { Technology Maturity: } \\
\square \quad \text { R and D } \\
\square \quad \text { Bench Scale } \\
\square \quad \text { Pilot Scale } \\
\square \quad \text { Commercial } \\
\text { Pick the best one }\end{array}$ & \multirow{3}{*}{ 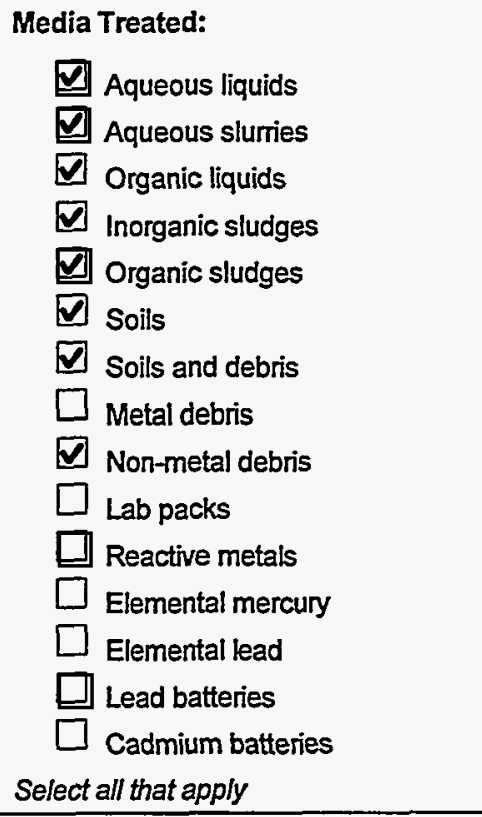 } & $\begin{array}{l}\text { MW Categories Accepted: } \\
\square \text { Low level waste } \\
\square \text { Transuranic waste } \\
\text { High level waste } \\
\text { Select all that apply }\end{array}$ \\
\hline$\square$ DOE/Gov experience & & Residuals: \\
\hline $\begin{array}{l}\text { Technology Availability: } \\
\text { Cumently available } \\
\text { Scheduled availability } \\
\text { Pick the best one } \\
\text { Scheduled Date: }\end{array}$ & & $\begin{array}{l}\square] \text { Residuals ready for } \\
\text { disposal } \\
\square \text { Residuals need } \\
\text { additional treatment } \\
\square \text { Secondary wastes } \\
\text { retumed to generator } \\
\text { Select all that apply }\end{array}$ \\
\hline
\end{tabular}


MIXED WASTE TREATABILITY STUDY TECHNOLOGY QUESTIONAIRE

\begin{tabular}{|c|c|c|}
\hline $\begin{array}{l}\text { Technology Name: } \\
\text { Plasma-Driven Reactor }\end{array}$ & $\begin{array}{l}\text { Technology Category: } \\
\text { Vitrification }\end{array}$ & \\
\hline \multicolumn{2}{|l|}{$\begin{array}{l}\text { Company Name and Address: } \\
\text { Scientific Ecology Group, Inc. } \\
\text { P.O. Box } 2530 \\
1560 \text { Bear Creek Road } \\
\text { Oak Ridge, TN } 37830\end{array}$} & $\begin{array}{l}\text { Contact: } \\
\text { Dr. Tom Snyder } \\
\text { Phone: } \\
\text { (423) } 376-8321 \\
\text { Fax: }\end{array}$ \\
\hline $\begin{array}{l}\text { Age of Company: } \\
10 \text { years }\end{array}$ & $\begin{array}{l}\text { Number of Employees: } \\
1300\end{array}$ & $\begin{array}{l}\text { Annual Revenue: } \\
\qquad \$ 150 \mathrm{M}\end{array}$ \\
\hline \multirow{3}{*}{\multicolumn{2}{|c|}{$\begin{array}{l}\text { Facility Name and Address: } \\
\text { Scientific Ecology Group, Inc. } \\
1560 \text { Bear Creek Rd. } \\
\text { Oak Ridge, TN } 37830\end{array}$}} & Age of Facility: \\
\hline & & $\begin{array}{l}\text { RCRA Treatment Permit } \\
\text { Date: } \quad \text { Exp: }\end{array}$ \\
\hline & & Rad Mat \#: R-73016-F96 \\
\hline \multicolumn{3}{|c|}{$\begin{array}{l}\text { Technology Description: } \\
\text { SEG has adapted a commercially available plasma-driven reactor to process LLRW. The Plasma Torch Waste Processing } \\
\text { Facility processes drummed, high organic wastes, slag, refractories, EAF dust, soils, burial wastes, reprocessing wastes, } \\
\text { salts, metals, and even chemicalbiological warfare wastes, using localized temperatures of up to } 5,000 \text { degrees C. At this } \\
\text { temperature, the hazardous chemistry is decoupled from the waste. Reaction and stabilization occur in a single step. The } \\
\text { process uses plasma, a high temperature, ionized, conductive gas created within the plasma torch by the interaction of a } \\
\text { gas with an electrical arc. This interaction disassociates the gas into electrons and ions which enables the gas to become } \\
\text { both thermally and electrically conductive. Plasma systems offer a means of achieving the high temperatures required for } \\
\text { the safe destruction of many toxic and hazardous wastes, including PCBs, dioxin, DDT, furans, halogenated hydrocarbons, } \\
\text { and RCRA wastes which, untreated, pose serious problems to the environment and to the public. }\end{array}$} \\
\hline $\begin{array}{ll}\text { System } & 160 \mathrm{lbs} / \mathrm{hr} \\
\text { Capacity: } & 266,240 \mathrm{lbs} / \text { annually }\end{array}$ & $\begin{array}{l}\text { Excess } \\
\text { Capacity: }\end{array}$ & \\
\hline $\begin{array}{l}\text { Technology Maturity: } \\
\square \quad \text { R and D } \\
\square \quad \text { Bench Scale } \\
\square \quad \text { Pilot Scale } \\
\square \quad \text { Commercial } \\
\text { Pick the best one }\end{array}$ & \multirow{3}{*}{$\begin{array}{l}\text { Media Treated: } \\
\square \text { Aqueous liquids } \\
\square \text { Aqueous slurries } \\
\square \text { Organic liquids } \\
\square \text { Inorganic sludges } \\
\square \text { Organic sludges } \\
\square \text { Soils } \\
\square \text { Soils and debris } \\
\square \text { Metal debris } \\
\square \text { Non-metal debris } \\
\square \text { Lab packs } \\
\square \text { Reactive metals } \\
\square \text { Elemental mercury } \\
\square \text { Elemental lead } \\
\square \text { Lead batteries } \\
\square \text { Cadmium batteries } \\
\text { Select all that apply }\end{array}$} & $\begin{array}{l}\text { MW Categories Accepted: } \\
\square \text { Low level waste } \\
\square \text { Transuranic waste } \\
\text { High level waste } \\
\text { Select all that apply }\end{array}$ \\
\hline$\square$ DOE/Gov experience & & Residuals: \\
\hline $\begin{array}{l}\text { Technology Availability: } \\
\qquad \text { Currently available } \\
\square \text { Scheduled availability } \\
\text { Pick the best one } \\
\text { Scheduled Date: }\end{array}$ & & $\begin{array}{l}\square \text { Residuals need } \\
\text { additional treatment } \\
\square \text { Secondary wastes } \\
\text { returned to generator } \\
\text { Select all that apply }\end{array}$ \\
\hline
\end{tabular}




\section{MIXED WASTE TREATABILITY STUDY TECHNOLOGY QUESTIONAIRE}




MIXED WASTE TREATABILITY STUDY TECHNOLOGY QUESTIONAIRE

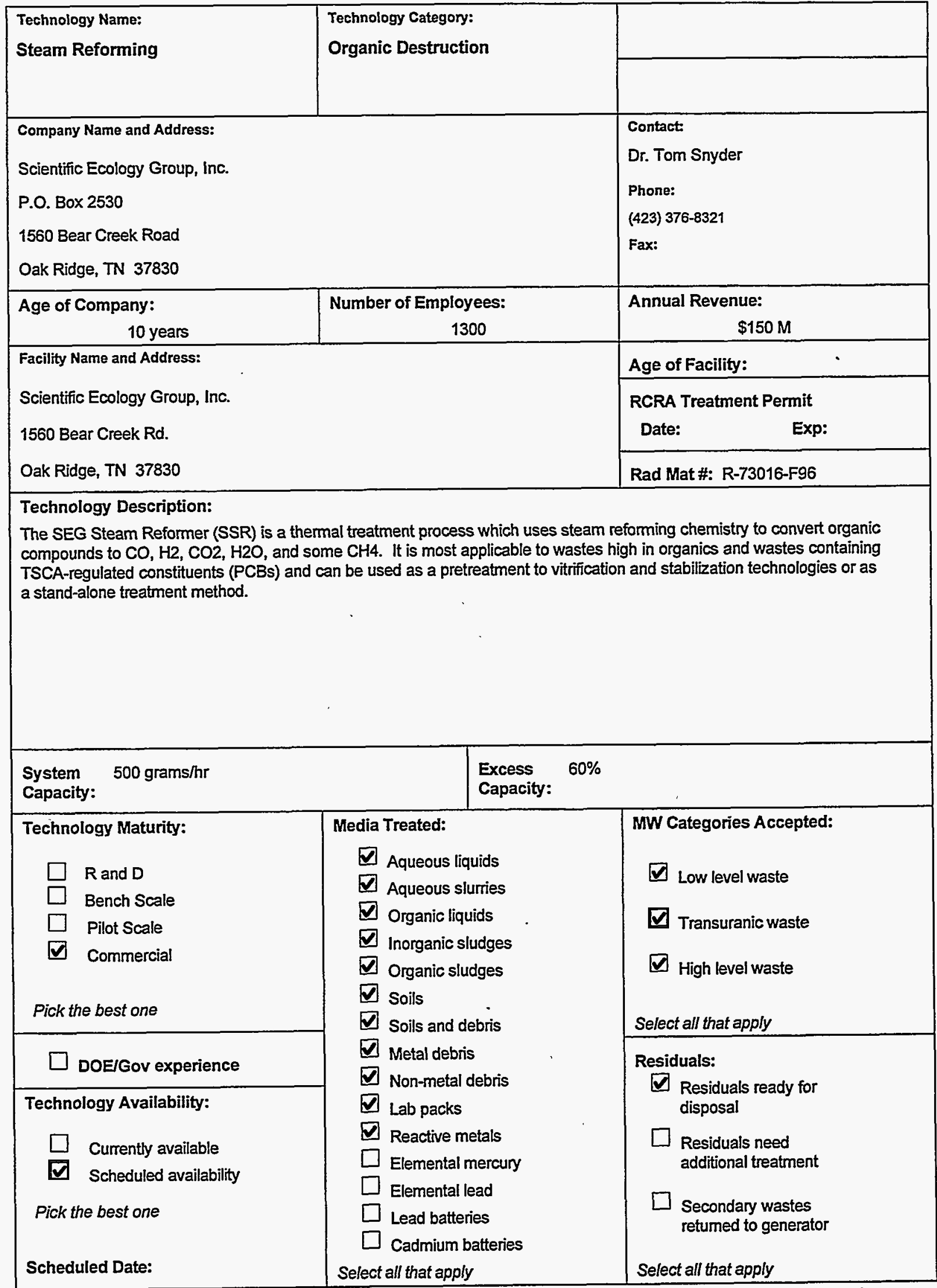


MIXED WASTE TREATABILITY STUDY TECHNOLOGY QUESTIONAIRE

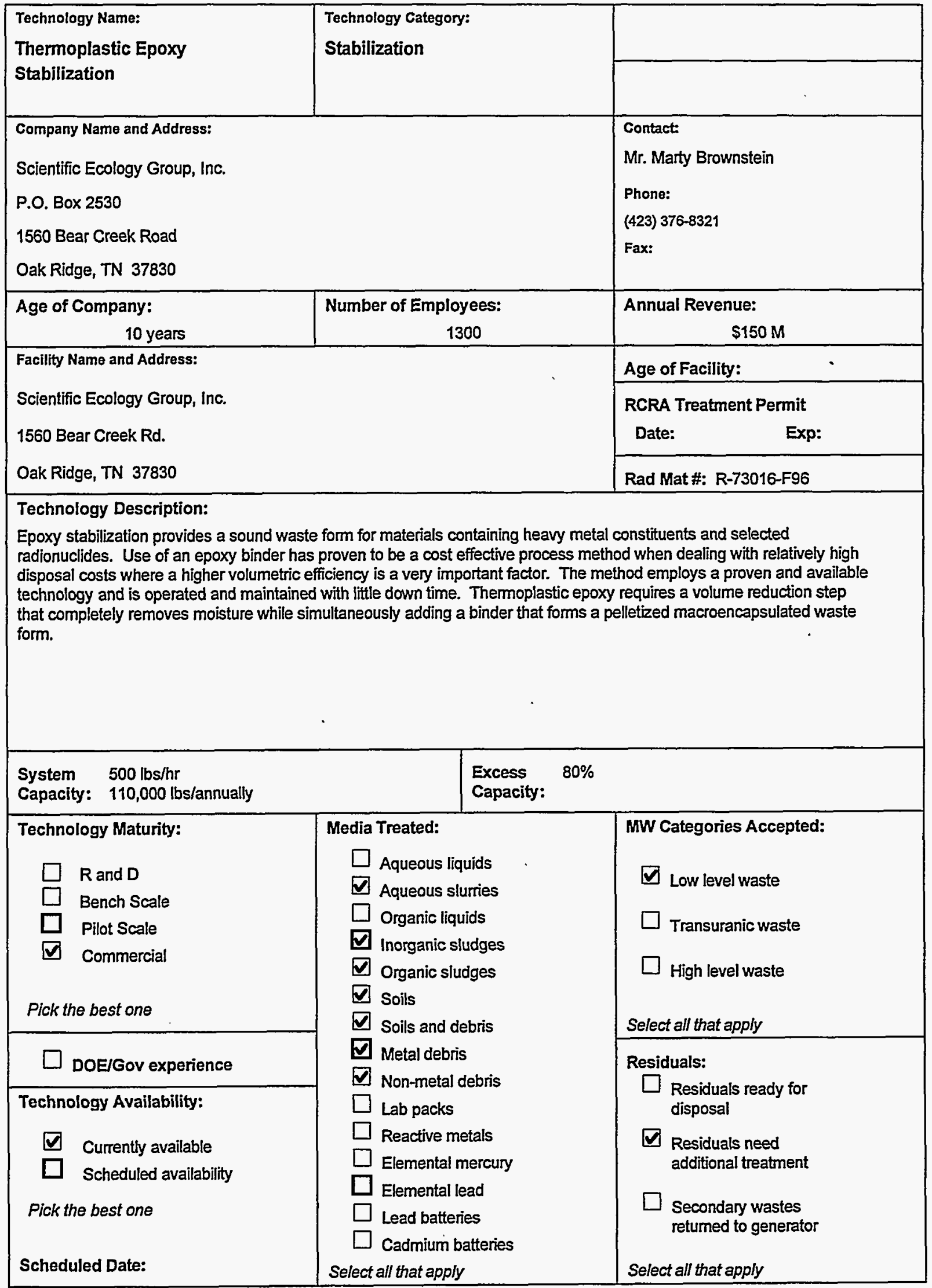


MIXED WASTE TREATABILITY STUDY TECHNOLOGY QUESTIONAIRE

\begin{tabular}{|c|c|c|}
\hline $\begin{array}{l}\text { Technology Name: } \\
\text { Thermoset Epoxy } \\
\text { Stabilization }\end{array}$ & $\begin{array}{l}\text { Technology Category: } \\
\text { Stabilization }\end{array}$ & \\
\hline \multicolumn{2}{|l|}{$\begin{array}{l}\text { Company Name and Address: } \\
\text { Scientific Ecology Group, Inc. } \\
\text { P.O. Box } 2530 \\
1560 \text { Bear Creek Road } \\
\text { Oak Ridge, TN } 37830\end{array}$} & $\begin{array}{l}\text { Contact: } \\
\text { Mr. Marty Brownstein } \\
\text { Phone: } \\
\text { (423) } 376-8321 \\
\text { Fax: }\end{array}$ \\
\hline $\begin{array}{l}\text { Age of Company: } \\
10 \text { years }\end{array}$ & $\begin{array}{r}\text { Number of Employees: } \\
1300 \\
\end{array}$ & $\begin{array}{l}\text { Annual Revenue: } \\
\qquad \$ 150 \mathrm{M}\end{array}$ \\
\hline \multirow{3}{*}{\multicolumn{2}{|c|}{$\begin{array}{l}\text { Facility Name and Address: } \\
\text { Scientific Ecology Group, Inc. } \\
1560 \text { Bear Creek Rd. } \\
\text { Oak Ridge, TN } 37830\end{array}$}} & Age of Facility: \\
\hline & & $\begin{array}{l}\text { RCRA Treatment Permit } \\
\text { Date: }\end{array}$ \\
\hline & & Rad Mat \#: R-73016-F96 \\
\hline \multicolumn{3}{|c|}{$\begin{array}{l}\text { Technology Description: } \\
\text { Epoxy stabilization provides a sound waste form for materials containing heavy metal constituents and selected } \\
\text { radionuclides. Use of an epoxy binder has proved to be a cost effective process method when dealing with relatively high } \\
\text { disposal costs where a higher volumetric efficiency is a very important factor. The method employs a proven and available } \\
\text { technology and is operated and maintained with little down time. Thermoset Epoxy stabilization requires a volume } \\
\text { reduction step that produces a dry material prior to stabilization. }\end{array}$} \\
\hline $\begin{array}{ll}\text { System } & 500 \mathrm{lbs} / \mathrm{hr} \\
\text { Capacity: } & 110,000 \mathrm{lbs} / \text { annually }\end{array}$ & $\begin{array}{l}\text { Excess } \\
\text { Capacity: }\end{array}$ & \\
\hline $\begin{array}{c}\text { Technology Maturity: } \\
\square \quad \text { R and D } \\
\square \text { Bench Scale } \\
\square \quad \text { Pilot Scale } \\
\square \quad \text { Commercial } \\
\text { Pick the best one }\end{array}$ & \multirow{3}{*}{$\begin{array}{l}\text { Media Treated: } \\
\square \text { Aqueous liquids } \\
\square \text { Aqueous slurries } \\
\square \text { Organic liquids } \\
\square \text { Inorganic sludges } \\
\square \text { Organic sludges } \\
\square \text { Soils } \\
\square \text { Soils and debris } \\
\square \text { Metal debris } \\
\square \text { Non-metal debris } \\
\square \text { Lab packs } \\
\square \text { Reactive metals } \\
\square \text { Elemental mercury } \\
\square \text { Elemental lead } \\
\square \text { Lead batteries } \\
\square \text { Cadmium batteries } \\
\text { Select all that apply }\end{array}$} & $\begin{array}{l}\text { MW Categories Accepted: } \\
\square \text { Low level waste } \\
\square \text { Transuranic waste } \\
\square \text { High level waste } \\
\text { Select all that apply }\end{array}$ \\
\hline$\square$ DOEIGov experience & & Residuals: \\
\hline $\begin{array}{l}\text { Technology Availability: } \\
\square \text { Currently available } \\
\square \text { Scheduled availability } \\
\text { Pick the best one } \\
\text { Scheduled Date: }\end{array}$ & & $\begin{array}{l}\square \text { Residuals need } \\
\text { additional treatment } \\
\square \text { Secondary wastes } \\
\text { retumed to generator } \\
\text { Select all that apply }\end{array}$ \\
\hline
\end{tabular}


MIXED WASTE TREATABILITY STUDY TECHNOLOGY QUESTIONAIRE

\begin{tabular}{|c|c|c|}
\hline $\begin{array}{l}\text { Technology Name: } \\
\text { Vinyl Ester Stabilization }\end{array}$ & $\begin{array}{l}\text { Technology Category: } \\
\text { Stabilization }\end{array}$ & \\
\hline \multicolumn{2}{|l|}{$\begin{array}{l}\text { Company Name and Address: } \\
\text { Scientific Ecology Group, Inc. } \\
\text { P.O. Box } 2530 \\
1560 \text { Bear Creek Road } \\
\text { Oak Ridge, TN } 37830\end{array}$} & $\begin{array}{l}\text { Contact: } \\
\text { Mr. Marty Brownstein } \\
\text { Phone: } \\
\text { (423) } 376-8321 \\
\text { Fax: }\end{array}$ \\
\hline $\begin{array}{r}\text { Age of Company: } \\
10 \text { years }\end{array}$ & $\begin{array}{c}\text { Number of Employees: } \\
1300\end{array}$ & $\begin{array}{r}\text { Annual Revenue: } \\
\$ 150 \mathrm{M} \\
\end{array}$ \\
\hline \multirow{3}{*}{\multicolumn{2}{|c|}{$\begin{array}{l}\text { Facility Name and Address: } \\
\text { Scientific Ecology Group, Inc. } \\
1560 \text { Bear Creek Rd. } \\
\text { Oak Ridge, TN } 37830\end{array}$}} & Age of Facility: \\
\hline & & $\begin{array}{l}\text { RCRA Treatment Permit } \\
\text { Date: } \quad \text { Exp: }\end{array}$ \\
\hline & & Rad Mat \#: R-73016-F96 \\
\hline \multicolumn{3}{|c|}{$\begin{array}{l}\text { Technology Description: } \\
\text { This stabilization process uses readily available chemicals that are easily handled in a safe manner. This system is a Dow } \\
\text { system based on a Dow proprietary process to form stable water-in-resin emulsions which are chemically cured to form } \\
\text { hard, solid monoliths. Liquid or slury waste is stirred with a low viscosity liquid solution of vinyl ester in styrene monomer } \\
\text { until a stable water-in-oil emulsion is formed. The mixture is cured by the addition of a free radical yielding peroxide } \\
\text { catalyst and a tertiary amine promoter. The final result is a dispersion of small spherical liquid particles (fine droplets) in a } \\
\text { continuous matrix of cured resin. The solidification process is relatively simple and works equally well for aqueous wastes } \\
\text { in the pH range from } 2.5 \text { to } 11.0 \text { and wastes containing dispersed solids such as ion exchange resins or filter aid materials. }\end{array}$} \\
\hline $\begin{array}{ll}\text { System } & 500 \mathrm{lbs} / \mathrm{hr} \\
\text { Capacity: } & 110,000 \mathrm{lbs} / \text { annually }\end{array}$ & $\begin{array}{l}\text { Excess } \quad 80 \% \\
\text { Capacity: }\end{array}$ & \\
\hline $\begin{array}{l}\text { Technology Maturity: } \\
\square \text { R and D } \\
\square \text { Bench Scale } \\
\square \text { Pilot Scale } \\
\square \text { Commercial } \\
\text { Pick the best one }\end{array}$ & \multirow{3}{*}{$\begin{array}{l}\text { Media Treated: } \\
\square \text { Aqueous liquids } \\
\square \text { Aqueous slurries } \\
\square \text { Organic liquids } \\
\square \text { Inorganic sludges } \\
\square \text { Organic sludges } \\
\square \text { Soils } \\
\square \text { Soils and debris } \\
\square \text { Metal debris } \\
\square \text { Non-metal debris } \\
\square \text { Lab packs } \\
\square \text { Reactive metals } \\
\square \text { Elemental mercury } \\
\square \text { Elemental lead } \\
\square \text { Lead batteries } \\
\square \text { Cadmium batteries } \\
\text { Select all that apply }\end{array}$} & $\begin{array}{l}\text { MW Categories Accepted: } \\
\square \text { Low level waste } \\
\square \text { Transuranic waste } \\
\square \text { High level waste } \\
\text { Select all that apply }\end{array}$ \\
\hline$\square$ DOEJGov experience & & Residuals: \\
\hline 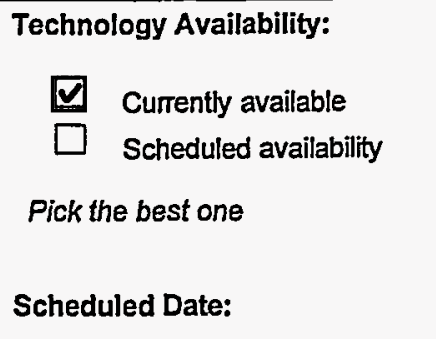 & & $\begin{array}{l}\square \text { Residuals need } \\
\text { additional treatment } \\
\square \text { Secondary wastes } \\
\text { retumed to generator } \\
\text { Select all that apply }\end{array}$ \\
\hline
\end{tabular}


MIXED WASTE TREATABILITY STUDY TECHNOLOGY QUESTIONAIRE

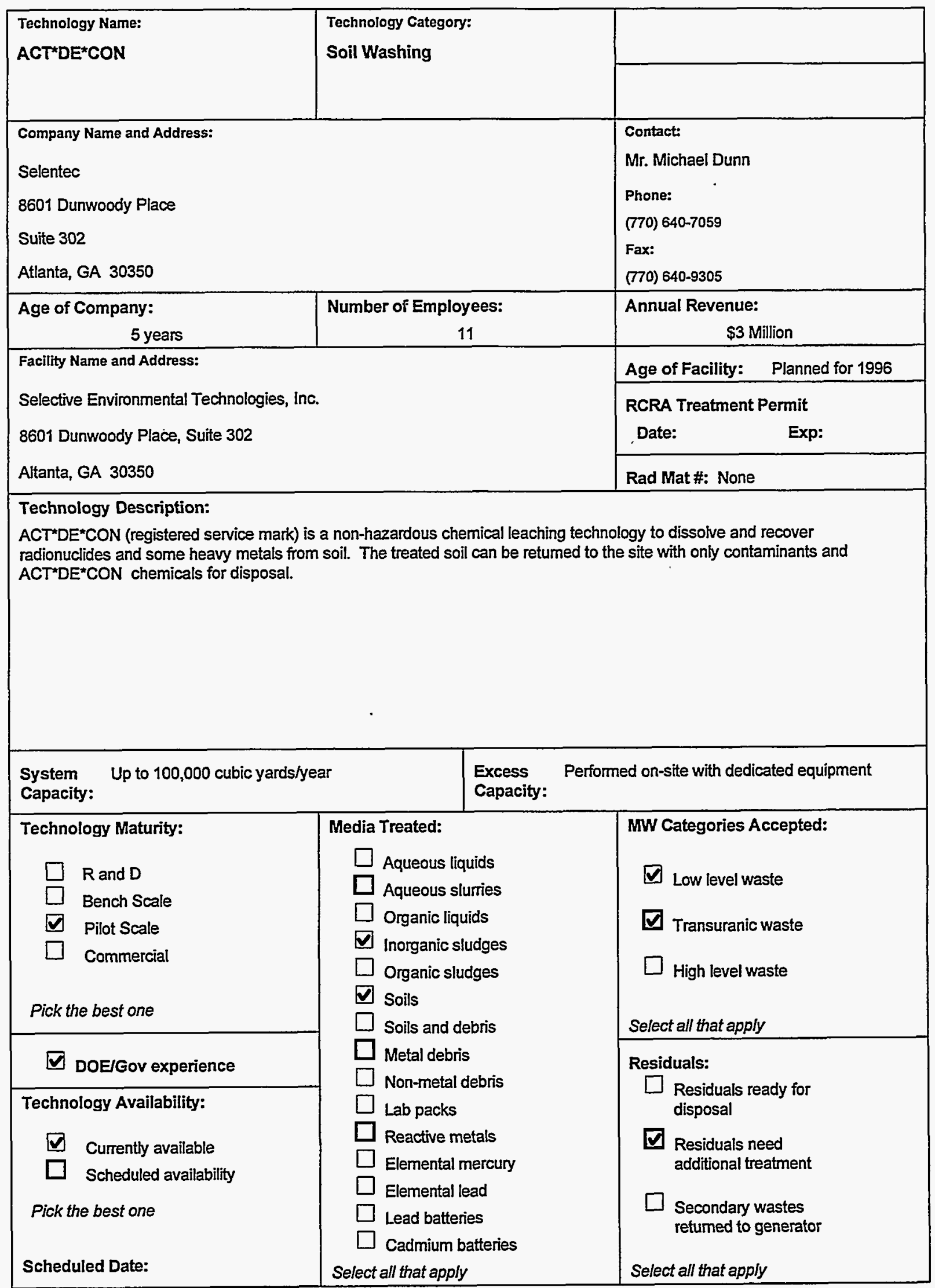




\section{MIXED WASTE TREATABILITY STUDY TECHNOLOGY QUESTIONAIRE}

\begin{tabular}{|c|c|c|}
\hline $\begin{array}{l}\text { Technology Name: } \\
\text { Electrochemical Ion } \\
\text { Exchange (EIX) }\end{array}$ & $\begin{array}{l}\text { Technology Category: } \\
\text { Extraction }\end{array}$ & \\
\hline $\begin{array}{l}\text { Company Name and Address: } \\
\text { Selentec } \\
8601 \text { Dunwoody Place } \\
\text { Suite } 302 \\
\text { Atlanta, GA } 30350\end{array}$ & & $\begin{array}{l}\text { Contact: } \\
\text { Mr. Michael Dunn } \\
\text { Phone: } \\
(770) 640-7059 \\
\text { Fax: } \\
(770) 640-9305\end{array}$ \\
\hline $\begin{array}{r}\text { Age of Company: } \\
\qquad 5 \text { years }\end{array}$ & $\begin{array}{c}\text { Number of Employees: } \\
11\end{array}$ & $\begin{array}{r}\text { Annual Revenue: } \\
\$ 3 \text { Million } \\
\end{array}$ \\
\hline \multirow{3}{*}{\multicolumn{2}{|c|}{$\begin{array}{l}\text { Facility Name and Address: } \\
\text { Selective Environmental Technologies, Inc. } \\
8601 \text { Dunwoody Place, Suite } 302 \\
\text { Altanta, GA } 30350\end{array}$}} & Age of Facility: Planned for 1996 \\
\hline & & $\begin{array}{l}\text { RCRA Treatment Permit } \\
\text { Date: } \quad \text { Exp: }\end{array}$ \\
\hline & & Rad Mat \#: None \\
\hline
\end{tabular}

Technology Description:

Electochemical Ion Exchange (EIX) selectively recovers and destroys nitrates from aqueous solutions. There is no waste product.

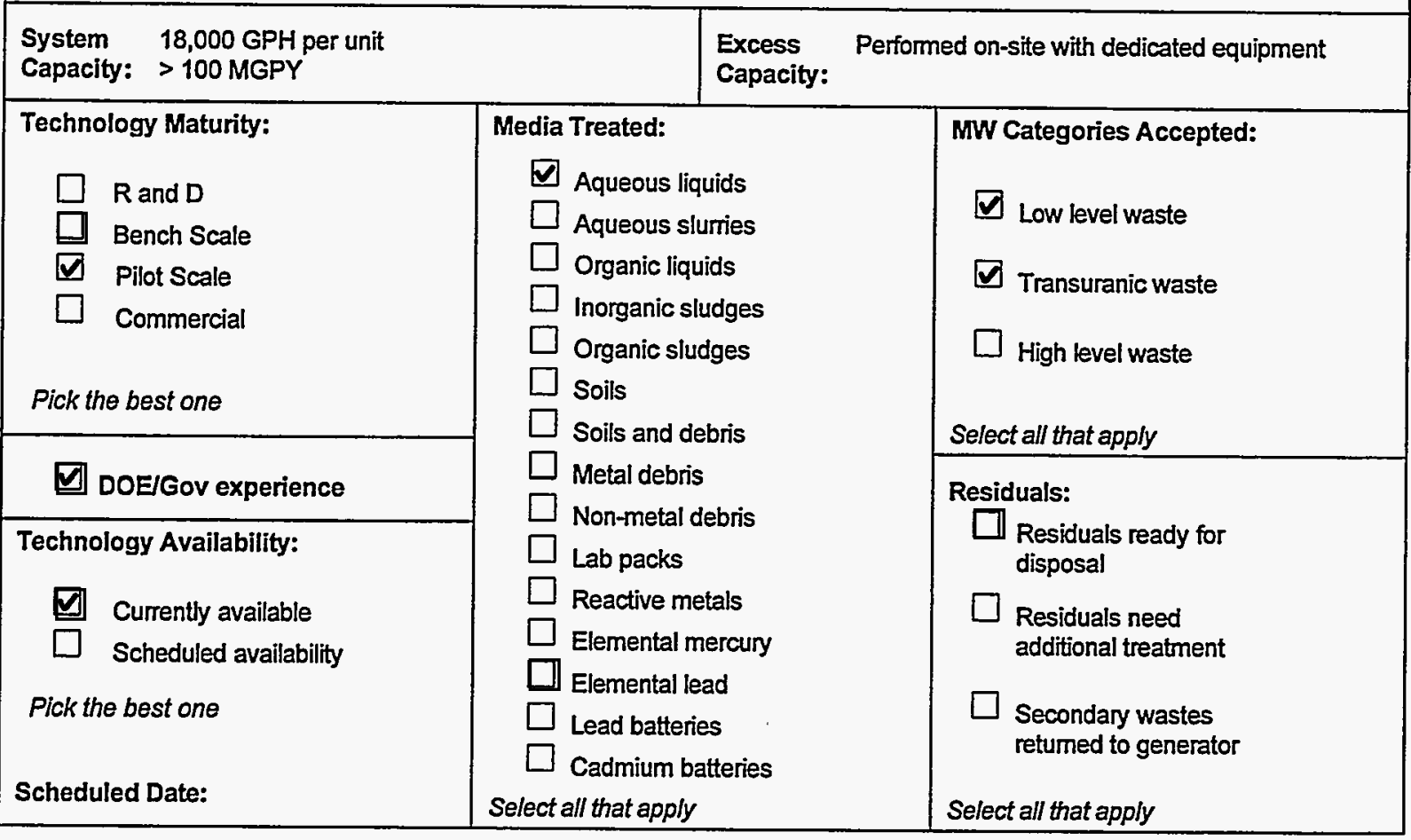




\section{MIXED WASTE TREATABILITY STUDY TECHNOLOGY QUESTIONAIRE}

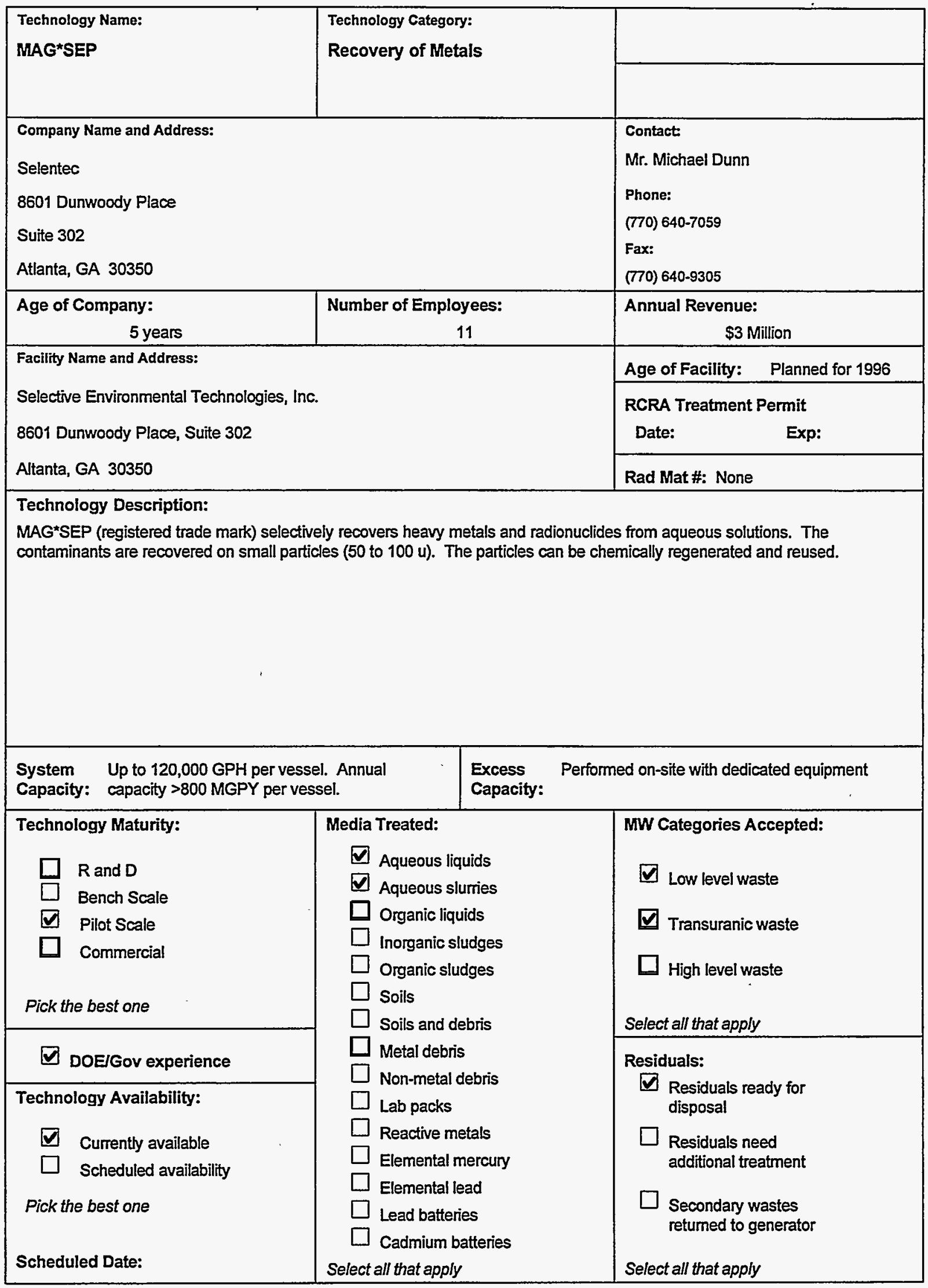




\section{MIXED WASTE TREATABILITY STUDY TECHNOLOGY QUESTIONAIRE}

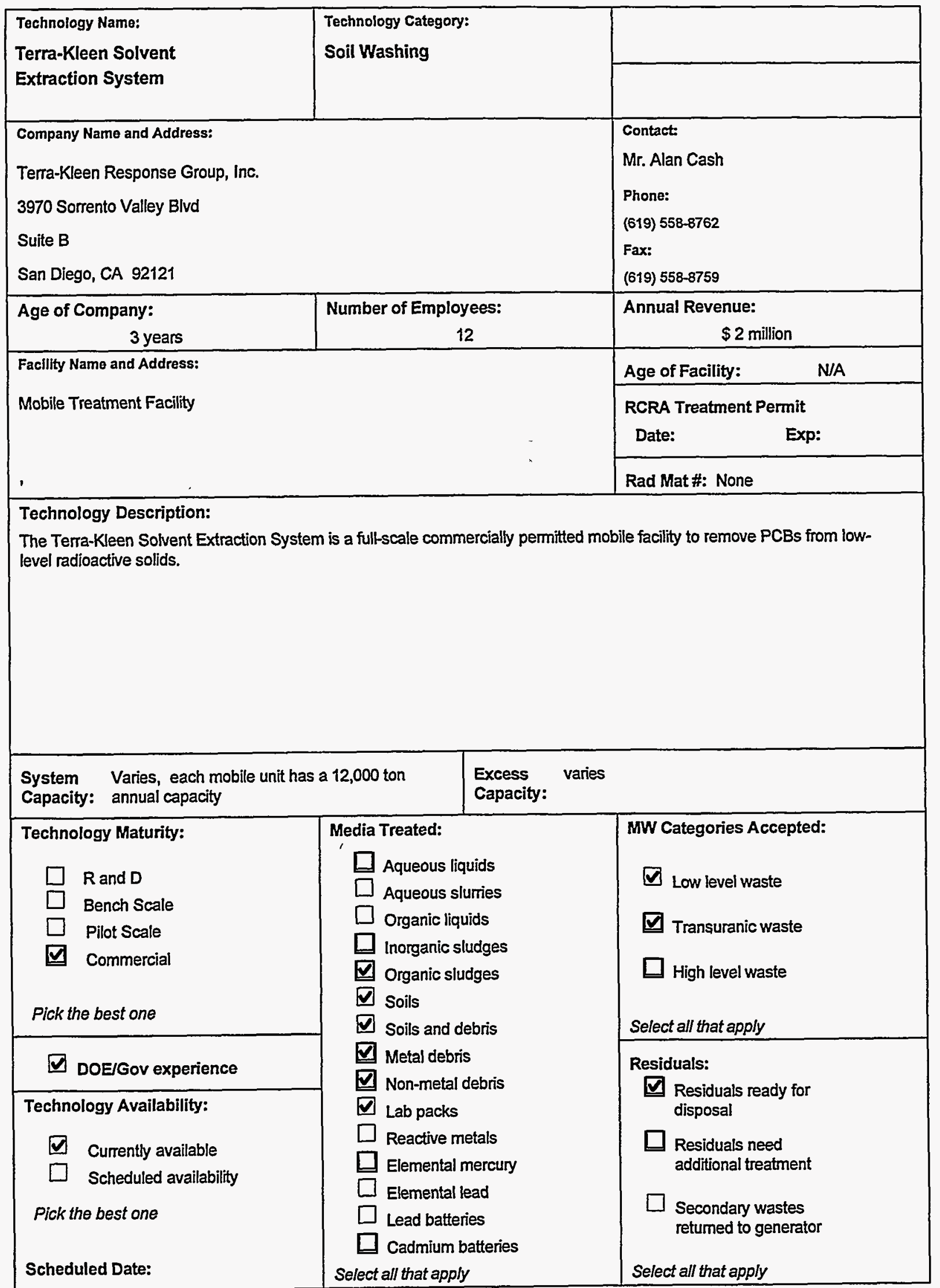


MIXED WASTE TREATABILITY STUDY TECHNOLOGY QUESTIONAIRE

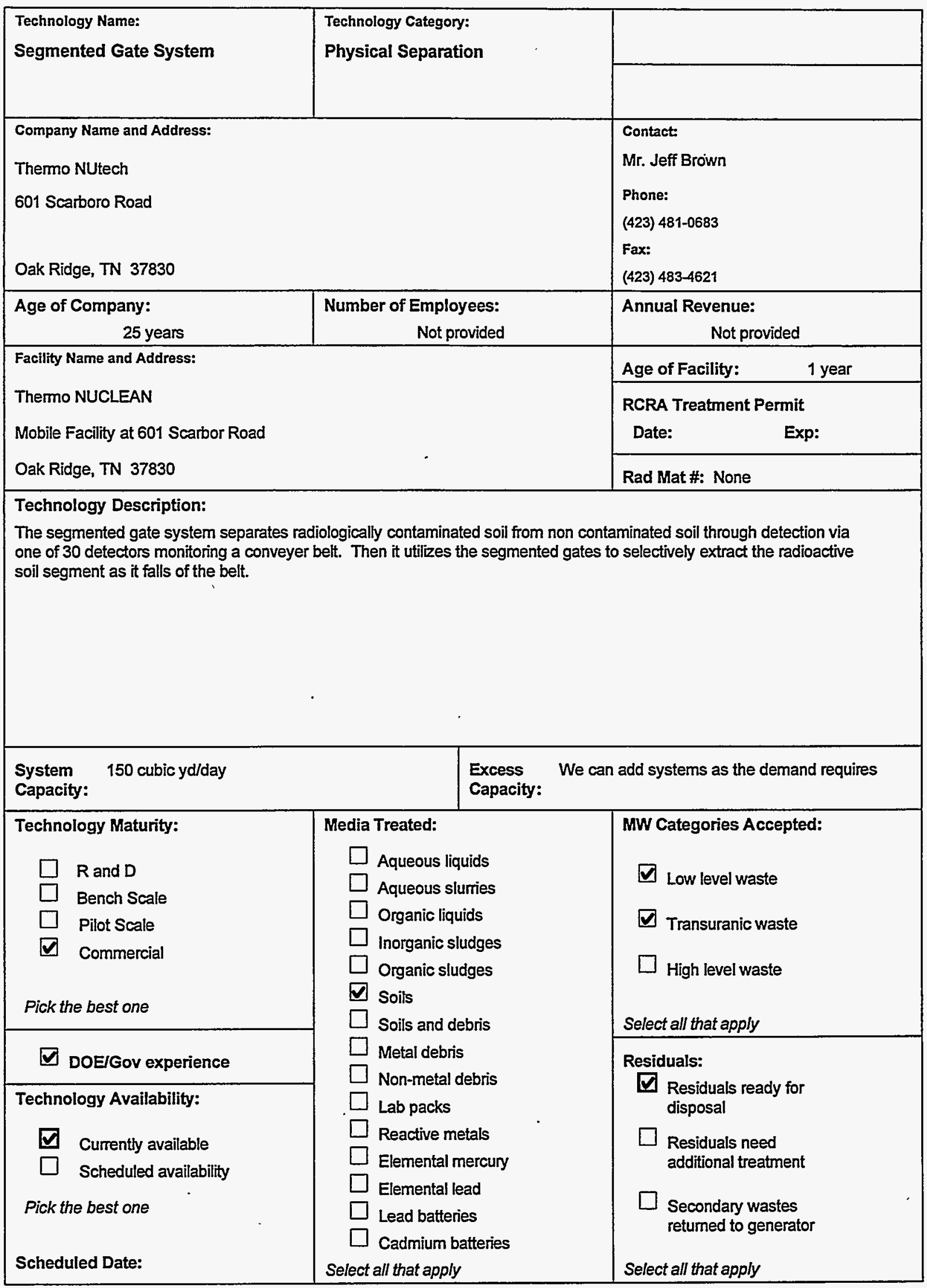


MIXED WASTE TREATABILITY STUDY TECHNOLOGY QUESTIONAIRE

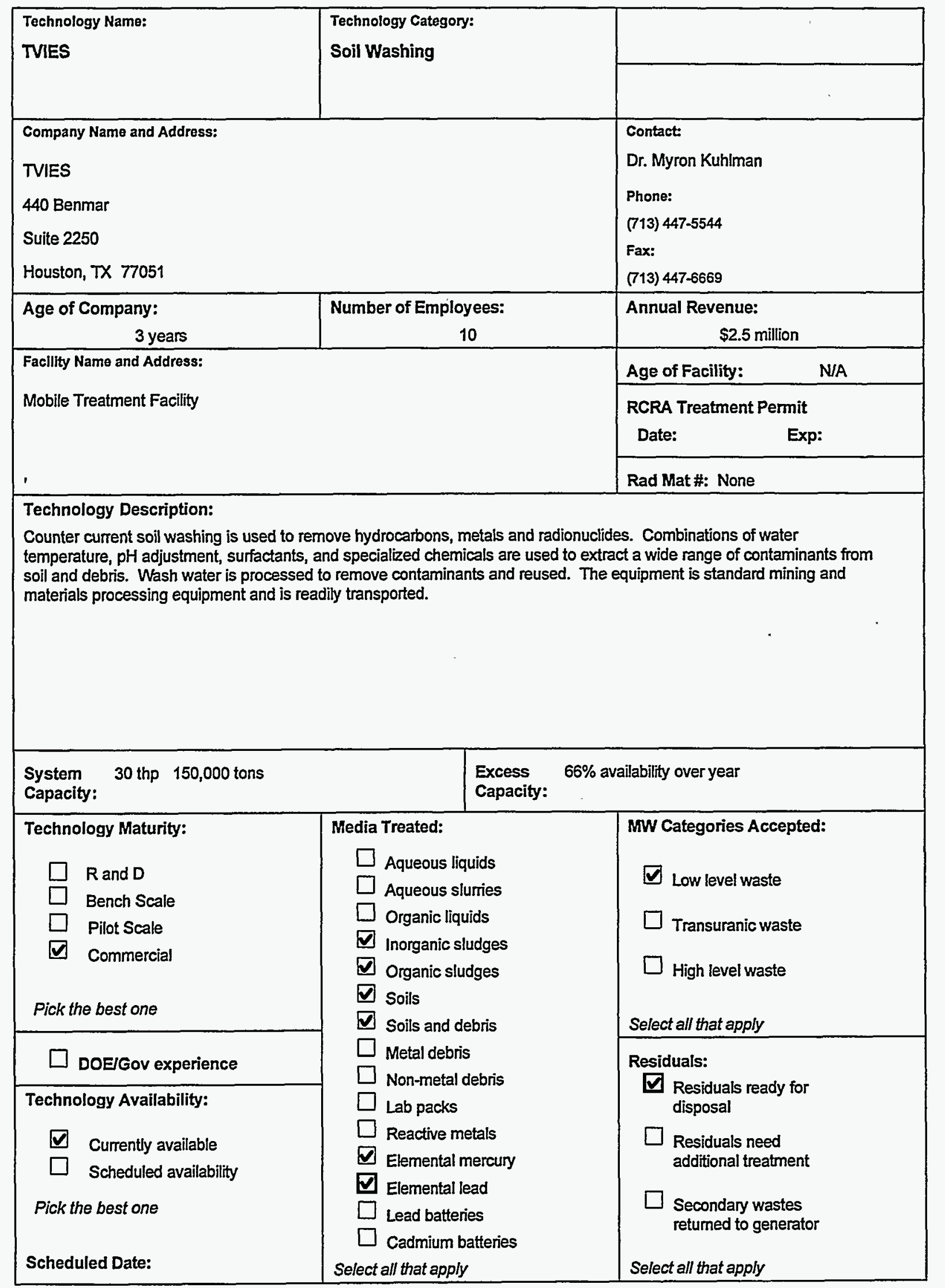


MIXED WASTE TREATABILITY STUDY TECHNOLOGY QUESTIONAIRE

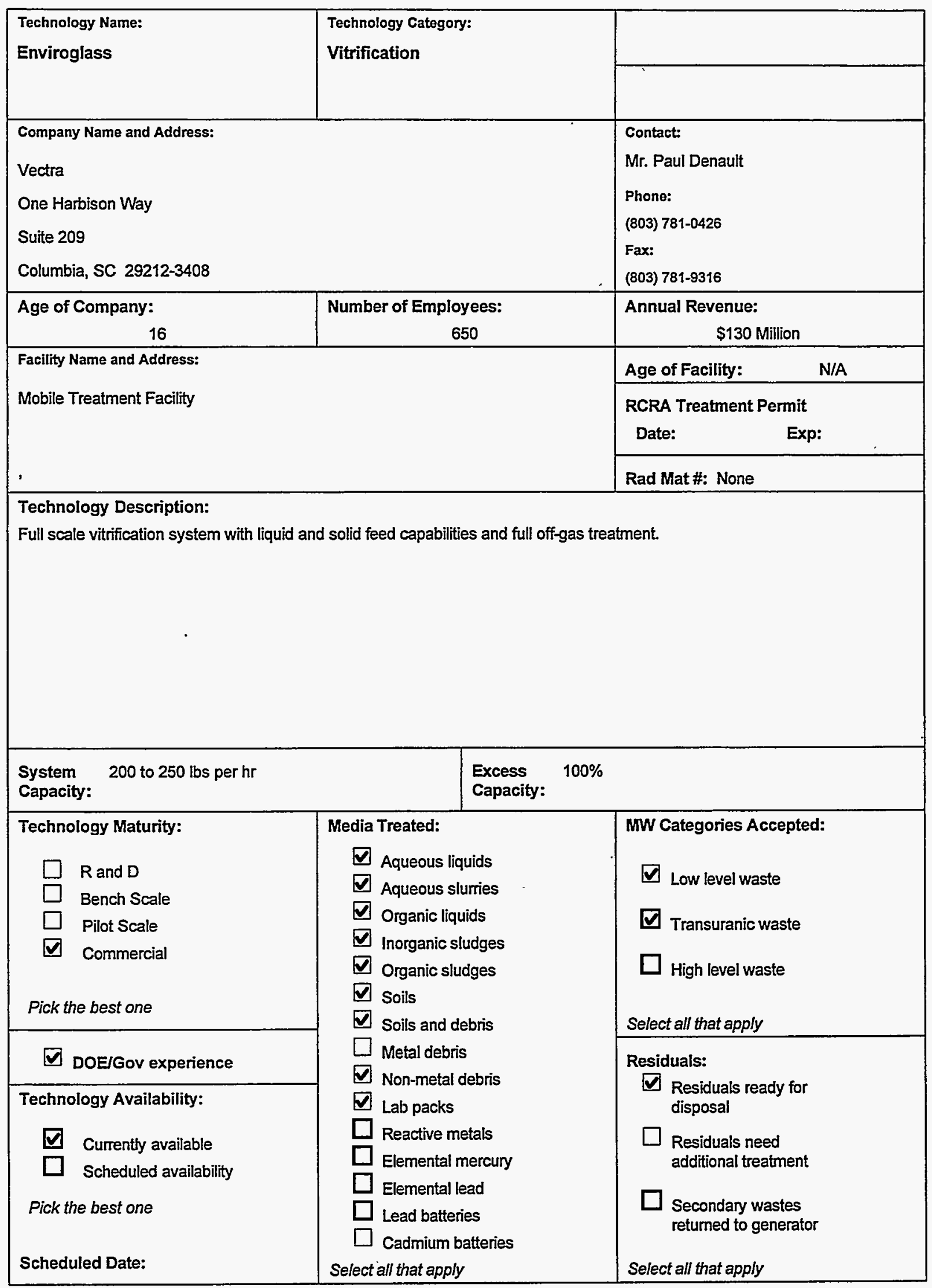


MIXED WASTE TREATABILITY STUDY TECHNOLOGY QUESTIONAIRE

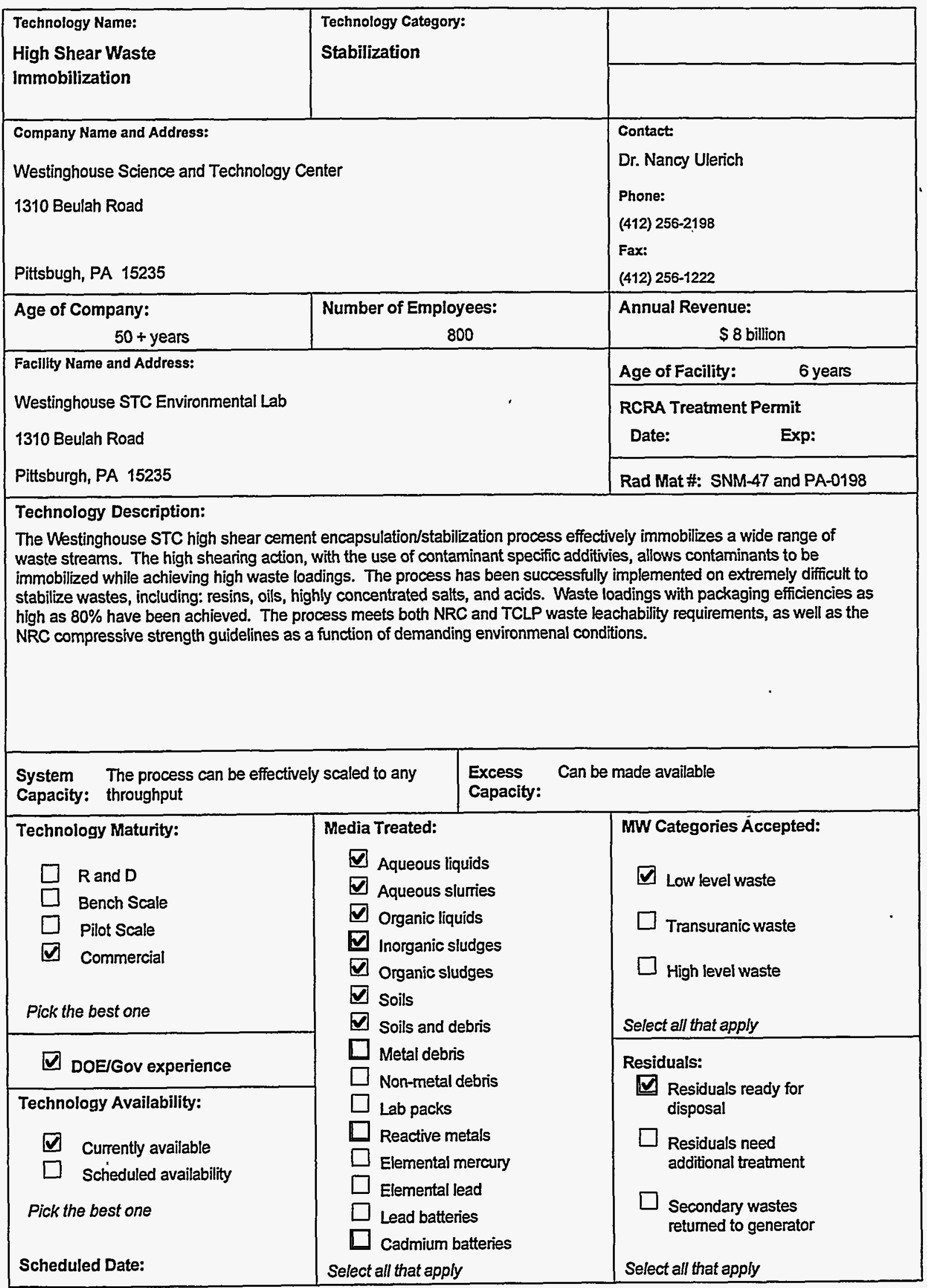


MIXED WASTE TREATABILITY STUDY TECHNOLOGY QUESTIONAIRE

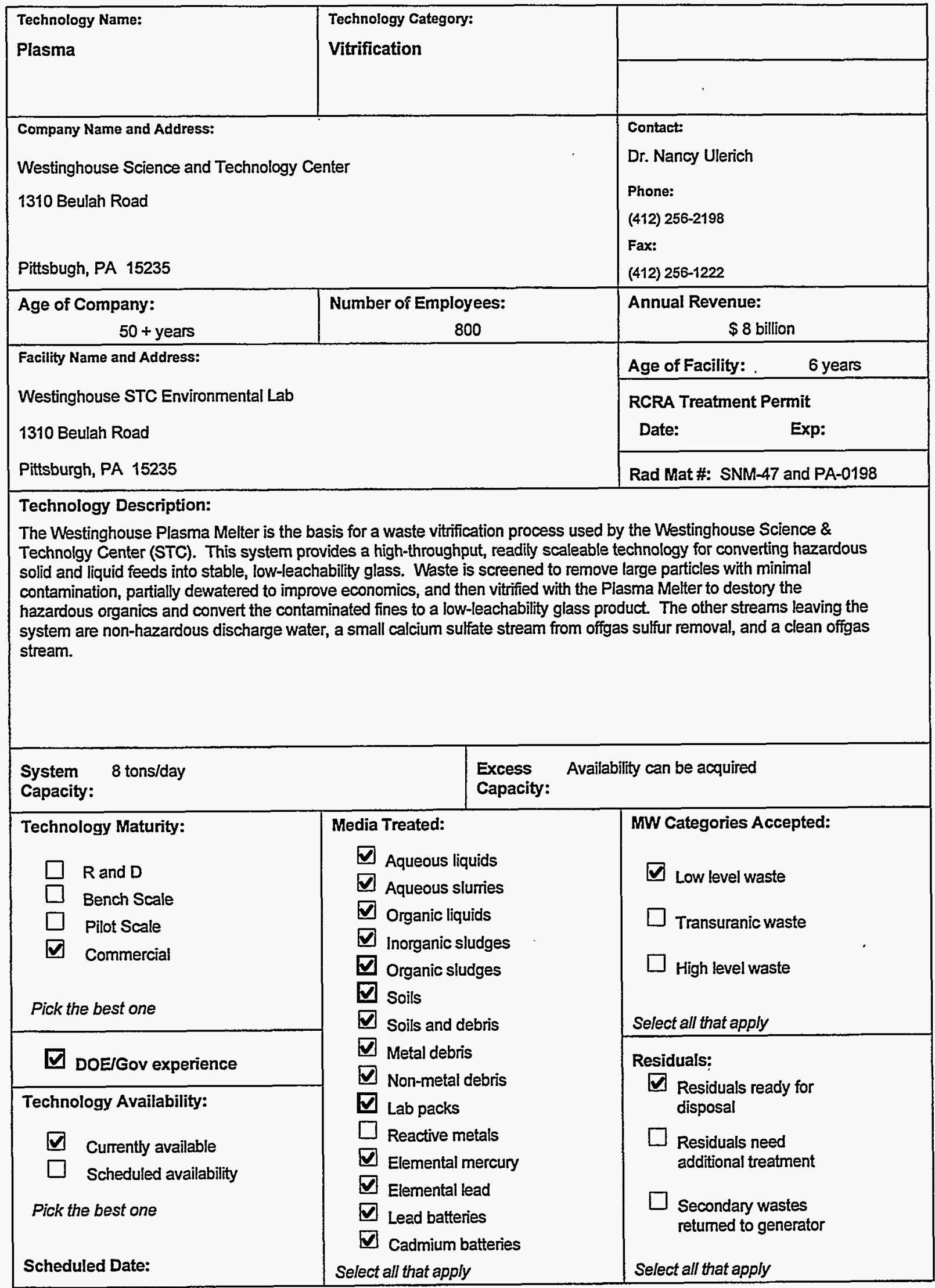


MIXED WASTE TREATABILITY STUDY TECHNOLOGY QUESTIONAIRE.

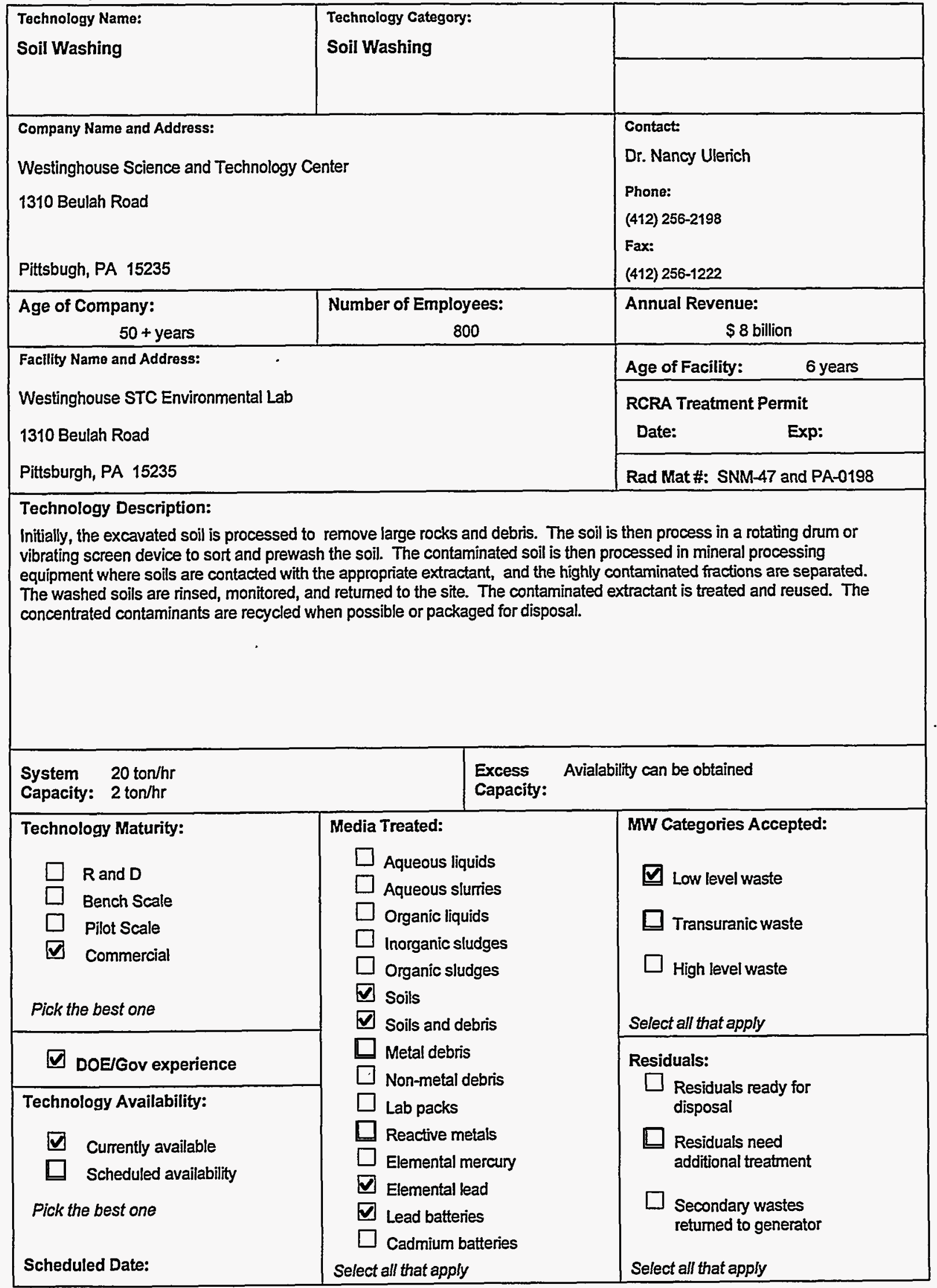


MIXED WASTE TREATABILITY STUDY TECHNOLOGY QUESTIONAIRE

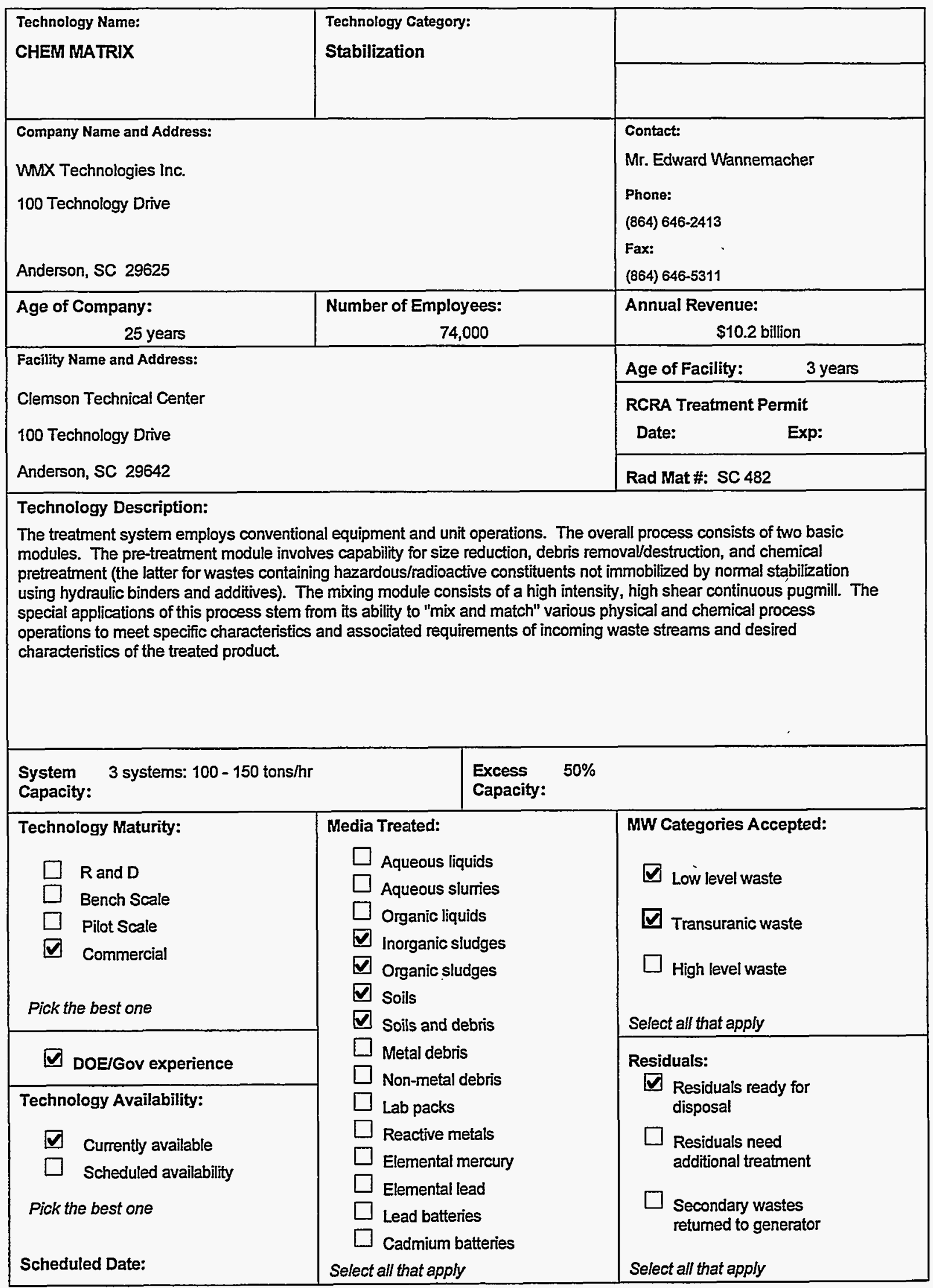


MIXED WASTE TREATABILITY STUDY TECHNOLOGY QUESTIONAIRE

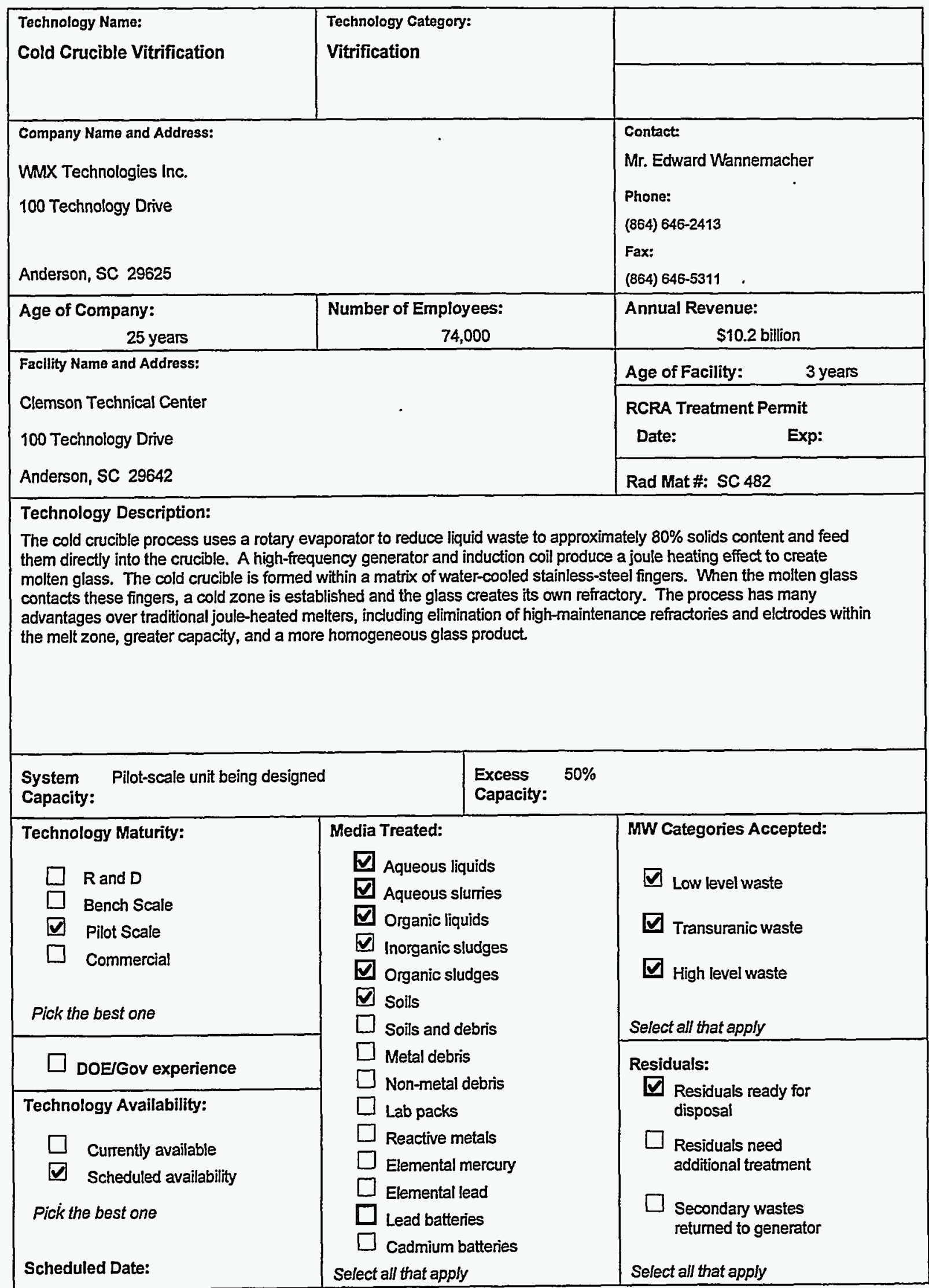


MIXED WASTE TREATABILITY STUDY TECHNOLOGY QUESTIONAIRE

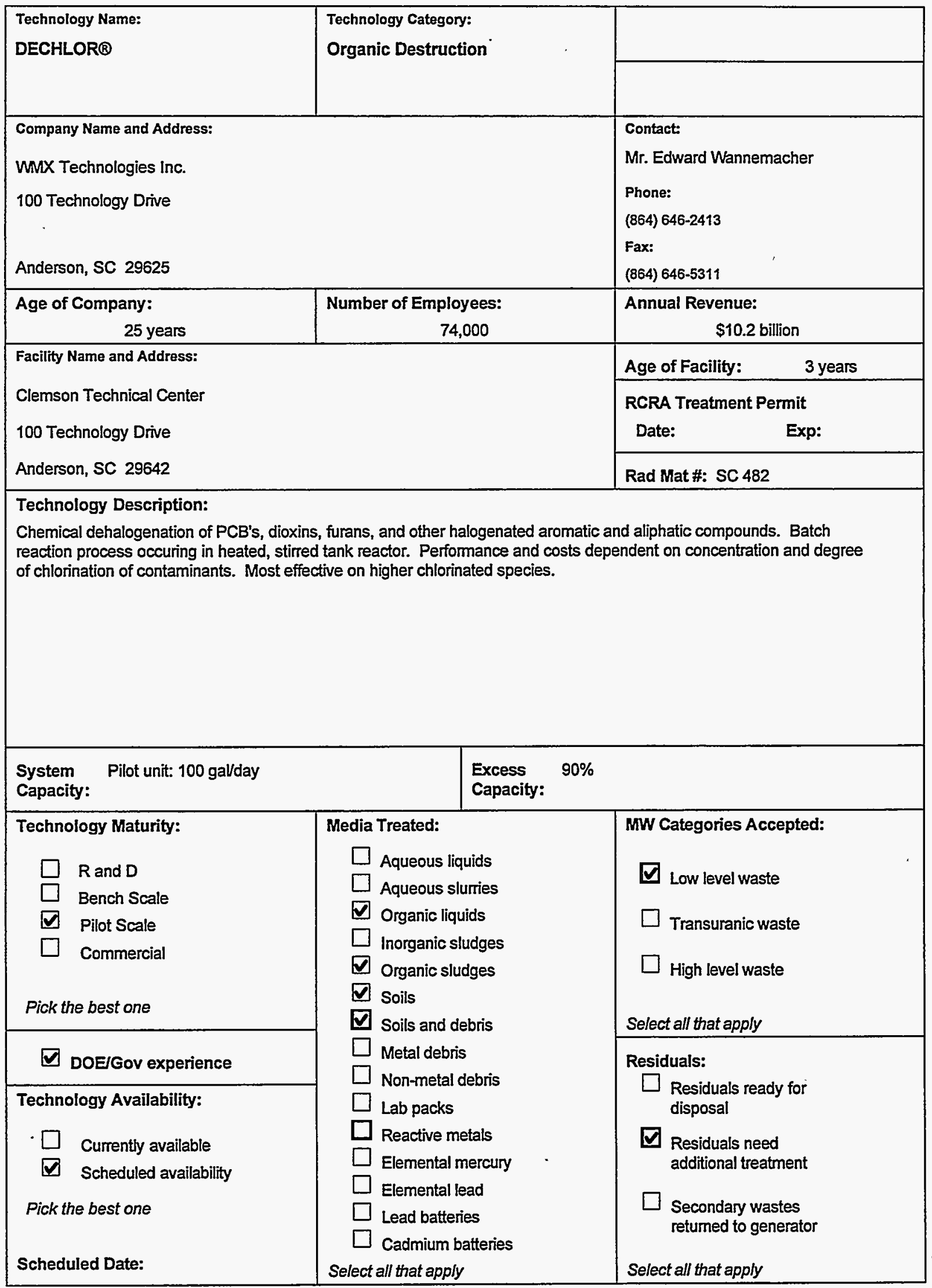


MIXED WASTE TREATABILITY STUDY TECHNOLOGY QUESTIONAIRE

\begin{tabular}{|c|c|c|}
\hline $\begin{array}{l}\text { Technology Name: } \\
\text { Freeze Separation }\end{array}$ & $\begin{array}{l}\text { Technology Category: } \\
\text { Recovery of Metals }\end{array}$ & \\
\hline $\begin{array}{l}\text { Company Name and Address: } \\
\text { WMX Technologies Inc. } \\
100 \text { Technology Drive } \\
\text { Anderson, SC } 29625\end{array}$ & & $\begin{array}{l}\text { Contact: } \\
\text { Mr. Edward Wannemacher } \\
\text { Phone: } \\
\text { (864) 646-2413 } \\
\text { Fax: } \\
\text { (864) 646-5311 }\end{array}$ \\
\hline $\begin{array}{l}\text { Age of Company: } \\
25 \text { years }\end{array}$ & $\begin{array}{r}\text { Number of Employees: } \\
74,000\end{array}$ & $\begin{array}{l}\text { Annual Revenue: } \\
\qquad \$ 10.2 \text { billion }\end{array}$ \\
\hline \multirow{3}{*}{\multicolumn{2}{|c|}{$\begin{array}{l}\text { Facility Name and Address: } \\
\text { Clemson Technical Center } \\
100 \text { Technology Drive } \\
\text { Anderson, SC } 29642\end{array}$}} & Age of Facility: $\quad 3$ years \\
\hline & & $\begin{array}{l}\text { RCRA Treatment Permit } \\
\text { Date: } \quad \text { Exp: }\end{array}$ \\
\hline & & Rad Mat \#: SC 482 \\
\hline \multicolumn{3}{|c|}{$\begin{array}{l}\text { Technology Description: } \\
\text { Freeze crystallization or separation reduces the volume of an aqueous solution by partial freezing of the water and } \\
\text { subsequent separation of the resulting solids (ice cyrstals). It offers significant advantage over traditional vapor-liquid } \\
\text { separation by providing much higher energy efficiencies. The technology is in commercial use for seawater desalinization } \\
\text { and food preservation. Only bench-scale testing has been done for mixed waste, primarily for radioactive waste containing } \\
\text { sodium and other nitrates. }\end{array}$} \\
\hline \multicolumn{2}{|c|}{$\begin{array}{l}\text { System Only bench-scale testing to date } \\
\text { Capacity: }\end{array}$} & $\begin{array}{l}\text { Excess } \\
\text { Capacity: }\end{array}$ \\
\hline $\begin{array}{ll}\square & R \text { and } D \\
\square & \text { Bench Scale } \\
\square & \text { Pilot Scale } \\
\square & \text { Commercial }\end{array}$ & \multirow{3}{*}{$\begin{array}{l}\text { Media Treated: } \\
\square \text { Aqueous liquids } \\
\square \text { Aqueous slurries } \\
\square \text { Organic liquids } \\
\square \text { Inorganic sludges } \\
\square \text { Organic sludges } \\
\square \text { Soils } \\
\square \text { Soils and debris } \\
\square \text { Metal debris } \\
\square \text { Non-metal debris } \\
\square \text { Lab packs } \\
\square \text { Reactive metals } \\
\square \text { Elemental mercury } \\
\square \text { Elemental lead } \\
\square \text { Lead batteries } \\
\square \text { Cadmium batteries } \\
\text { Select all that apply }\end{array}$} & $\begin{array}{l}\text { MW Categories Accepted: } \\
\square \text { Low level waste } \\
\square \text { Transuranic waste } \\
\square \text { High level waste } \\
\text { Select all that apply }\end{array}$ \\
\hline$\square$ DOE/Gov experience & & Residuals: \\
\hline $\begin{array}{l}\text { Technology Availability: } \\
\qquad \text { Currently available } \\
\square \text { Scheduled availability } \\
\text { Pick the best one } \\
\text { Scheduled Date: }\end{array}$ & & $\begin{array}{l}\text { Residuals ready for } \\
\text { disposal } \\
\text { Residuals need } \\
\text { additional treatment } \\
\text { Secondary wastes } \\
\text { retumed to generator } \\
\text { Select all that apply }\end{array}$ \\
\hline
\end{tabular}


MIXED WASTE TREATABILITY STUDY TECHNOLOGY QUESTIONAIRE

\begin{tabular}{|c|c|c|}
\hline $\begin{array}{l}\text { Technology Name: } \\
\text { Joule-Heated Melters }\end{array}$ & $\begin{array}{l}\text { Technology Category: } \\
\text { Vitrification }\end{array}$ & \\
\hline $\begin{array}{l}\text { Company Name and Address: } \\
\text { WMX Technologies Inc. } \\
100 \text { Technology Drive } \\
\text { Anderson, SC } 29625\end{array}$ & & $\begin{array}{l}\text { Contact: } \\
\text { Mr. Edward Wannemacher } \\
\text { Phone: } \\
\text { (864) } 646-2413 \\
\text { Fax: } \\
\text { (864) } 646-5311\end{array}$ \\
\hline $\begin{array}{r}\text { Age of Company: } \\
25 \text { years }\end{array}$ & $\begin{array}{l}\text { Number of Employees: } \\
\qquad 74,000\end{array}$ & $\begin{array}{l}\text { Annual Revenue: } \\
\qquad 10.2 \text { billion }\end{array}$ \\
\hline \multicolumn{2}{|l|}{ Facility Name and Address: } & Age of Facility: $\quad 3$ years \\
\hline \multicolumn{2}{|l|}{$\begin{array}{l}\text { Clemson Technical Center } \\
100 \text { Technology Drive }\end{array}$} & $\begin{array}{l}\text { RCRA Treatment Permit } \\
\text { Date: } \quad \text { Exp: }\end{array}$ \\
\hline \multicolumn{2}{|l|}{$\begin{array}{l}100 \text { Technology Drve } \\
\text { Anderson, SC } 29642\end{array}$} & Rad Mat \#: SC 482 \\
\hline \multicolumn{3}{|c|}{$\begin{array}{l}\text { As a member of the DOE Industrial Vitrification Center, Rust is performing bench-scale and pilot-scale demonstrations for } \\
\text { vitrifying commercial low-level waste from the nuclear utility industry, and has extended these investigations to include } \\
\text { mixed waste. Various vitrification processes are being tested. Two joule-heated vitrification systems are available for } \\
\text { demonstration at CTC or the IVC: an EnVitCo cold-top melter and a stirred glass melter (Stir Melter). }\end{array}$} \\
\hline $\begin{array}{l}\text { System Not provided } \\
\text { Capacity: }\end{array}$ & \multicolumn{2}{|c|}{$\begin{array}{l}\text { Excess Not provided } \\
\text { Capacity: }\end{array}$} \\
\hline 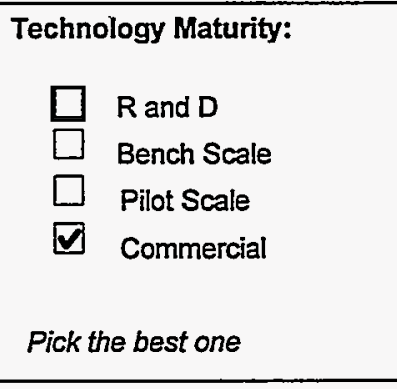 & \multirow{3}{*}{$\begin{array}{l}\text { Media Treated: } \\
\square \text { Aqueous liquids } \\
\square \text { Aqueous slurries } \\
\square \text { Organic liquids } \\
\square \text { Inorganic sludges } \\
\square \text { Organic sludges } \\
\square \text { Soils } \\
\square \text { Soils and debris } \\
\square \text { Metal debris } \\
\square \text { Non-metal debris } \\
\square \text { Lab packs } \\
\square \text { Reactive metals } \\
\square \text { Elemental mercury } \\
\square \text { Elemental lead } \\
\square \text { Lead batteries } \\
\square \text { Cadmium batteries } \\
\text { Select all that apply }\end{array}$} & $\begin{array}{l}\text { MW Categories Accepted: } \\
\square \text { Low level waste } \\
\square \text { Transuranic waste } \\
\square \text { High level waste } \\
\text { Select all that apply }\end{array}$ \\
\hline$\square$ DOEJGov experience & & Residuals: \\
\hline $\begin{array}{l}\text { Technology Availability: } \\
\square \text { Currently available } \\
\square \text { Scheduled availability } \\
\text { Pick the best one } \\
\text { Scheduled Date: }\end{array}$ & & $\begin{array}{l}\square \text { Residuals ready for } \\
\text { disposal } \\
\square \text { Residuals need } \\
\text { additional treatment } \\
\square \text { Secondary wastes } \\
\text { retumed to generator } \\
\text { Select all that apply }\end{array}$ \\
\hline
\end{tabular}




\section{MIXED WASTE TREATABILITY STUDY TECHNOLOGY QUESTIONAIRE}

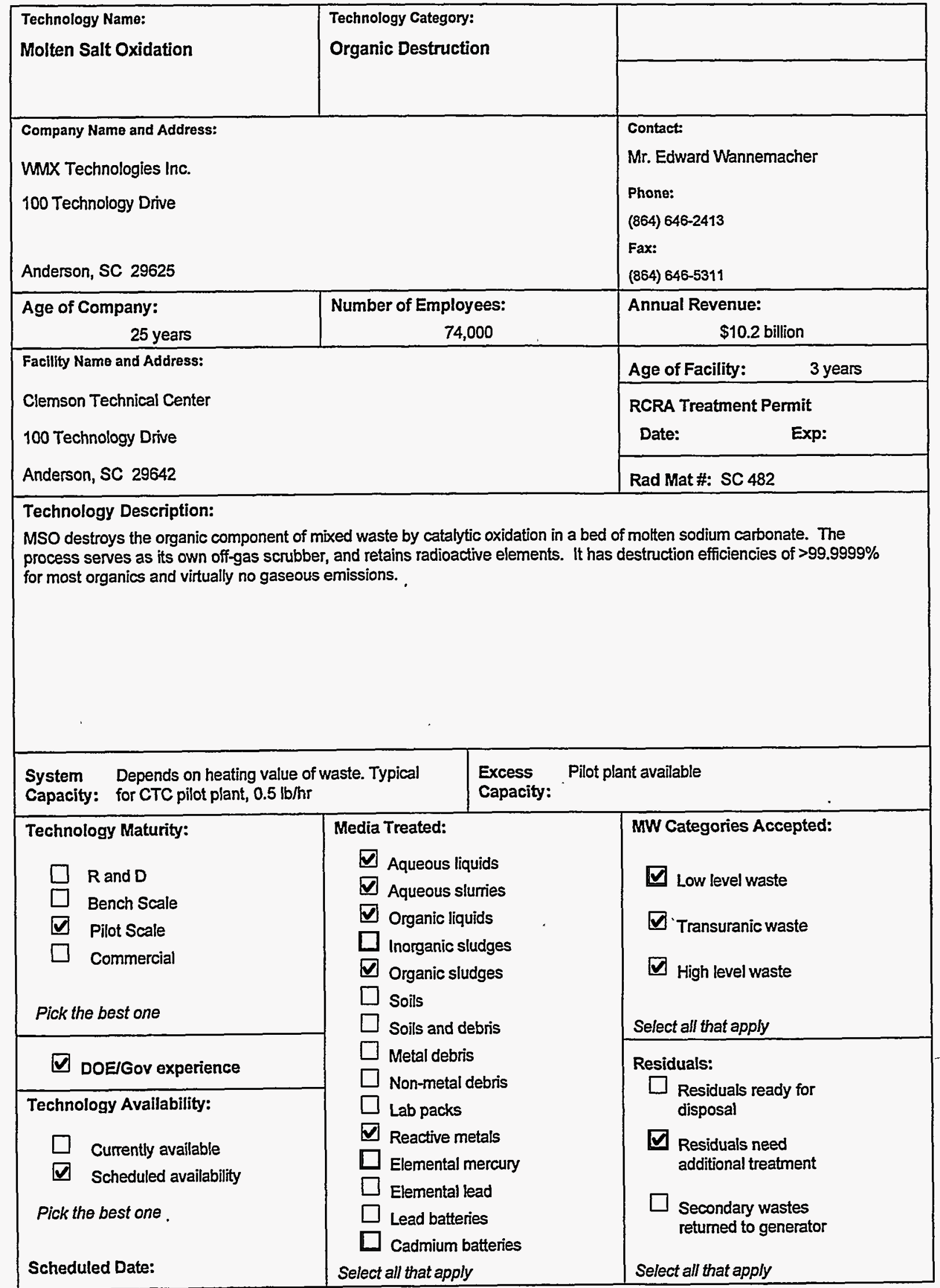


MIXED WASTE TREATABILITY STUDY TECHNOLOGY QUESTIONAIRE

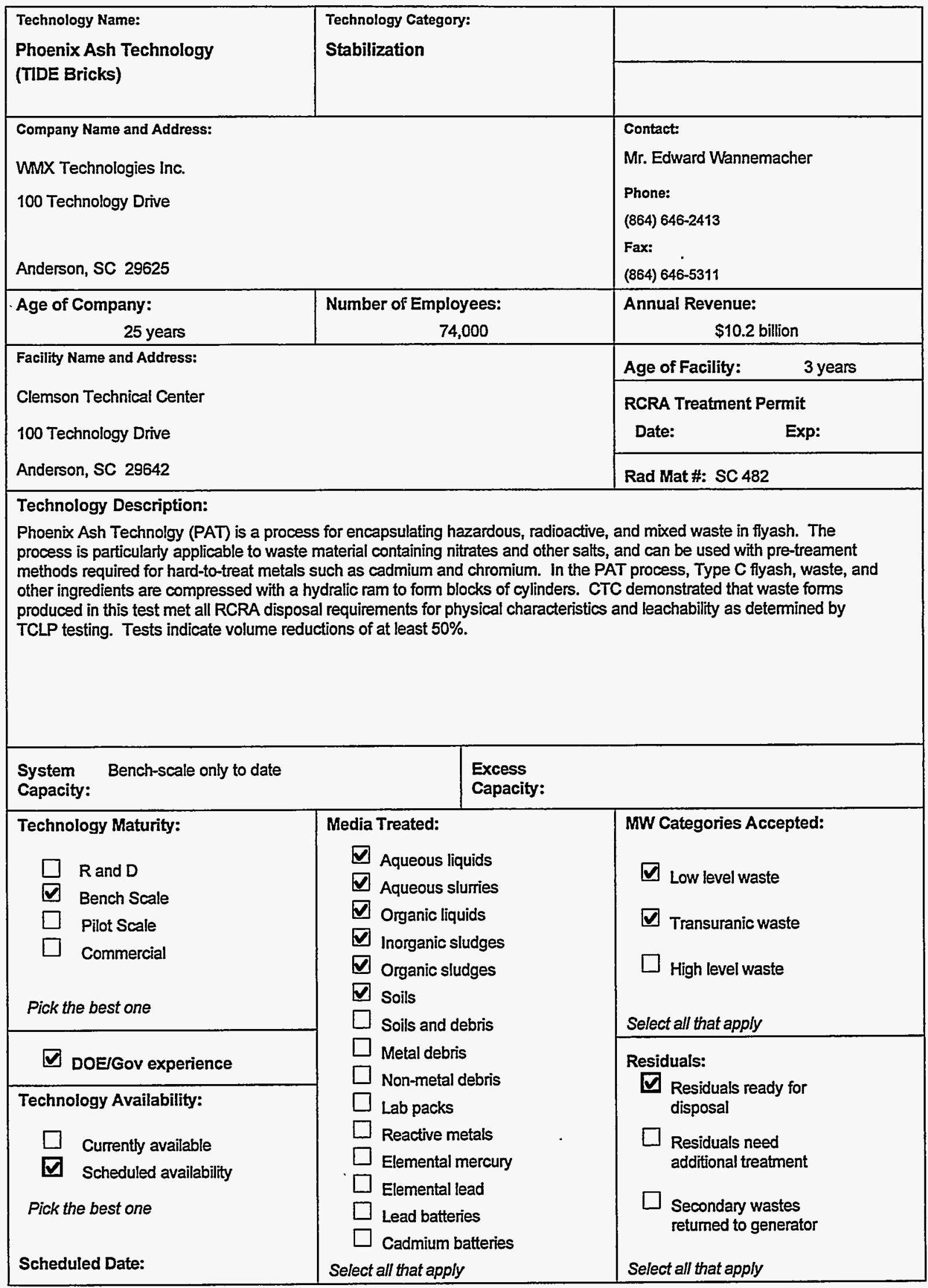


MIXED WASTE TREATABILITY STUDY TECHNOLOGY QUESTIONAIRE

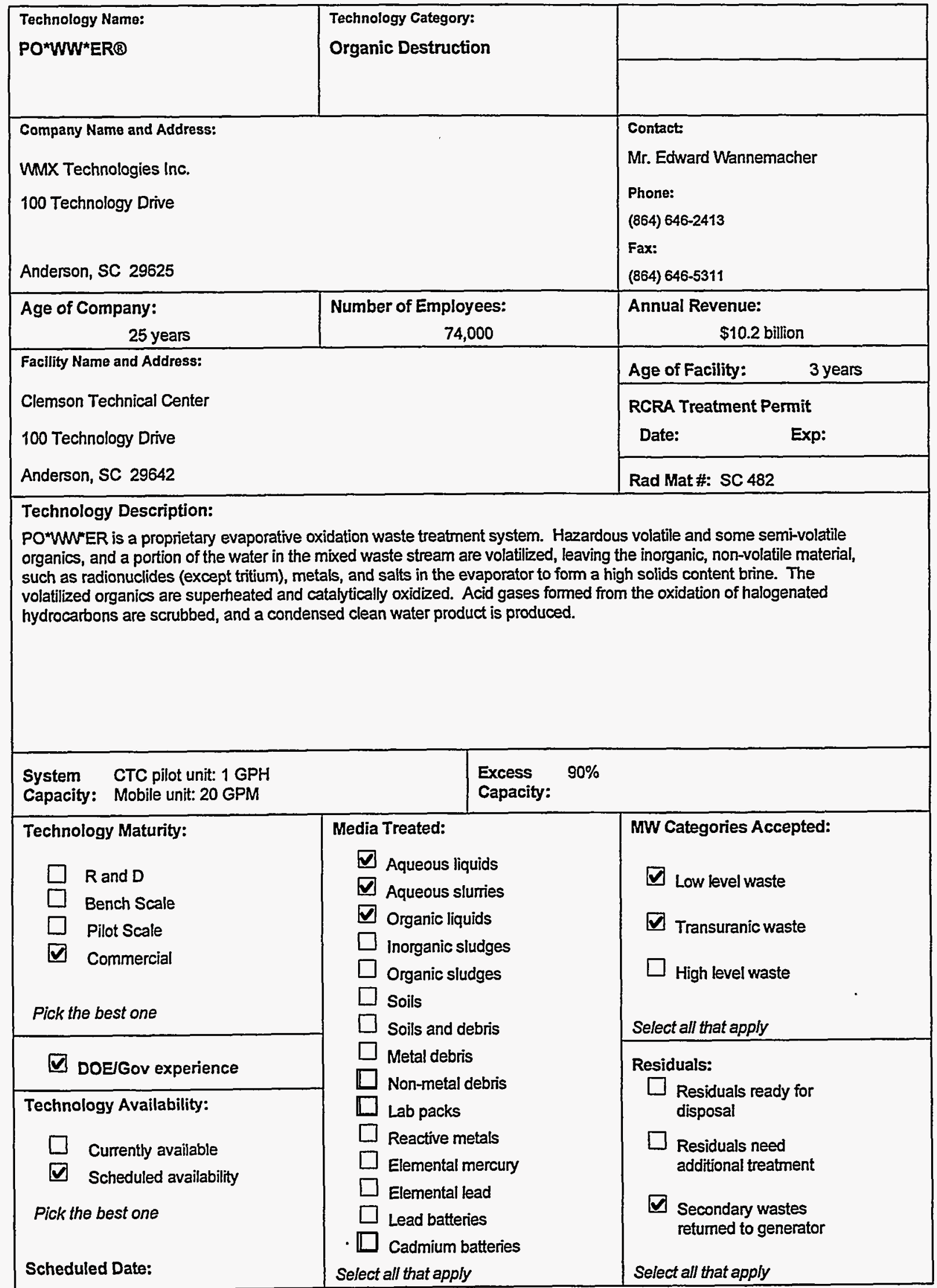


MIXED WASTE TREATABILITY STUDY TECHNOLOGY QUESTIONAIRE

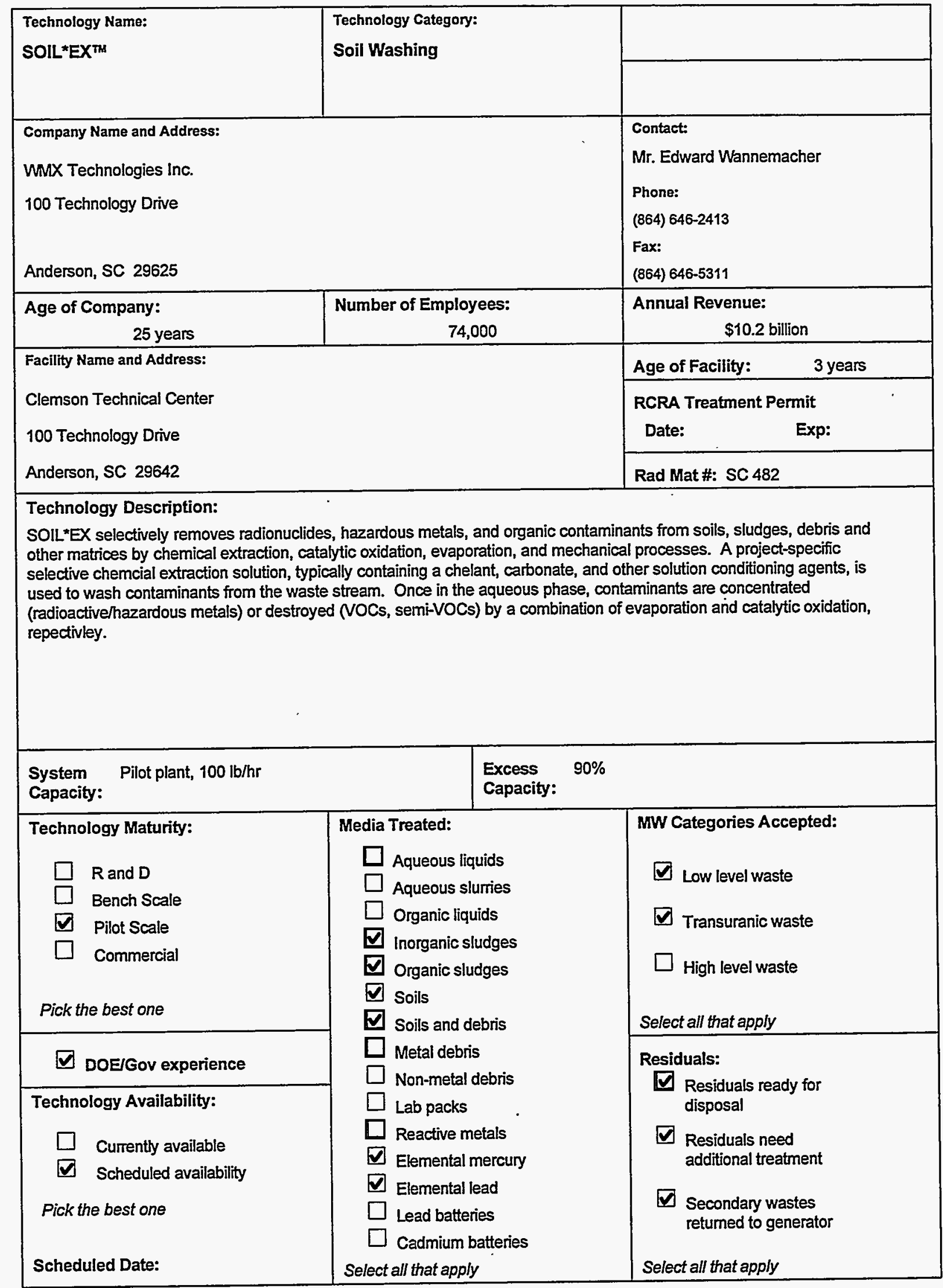


MIXED WASTE TREATABILITY STUDY TECHNOLOGY QUESTIONAIRE

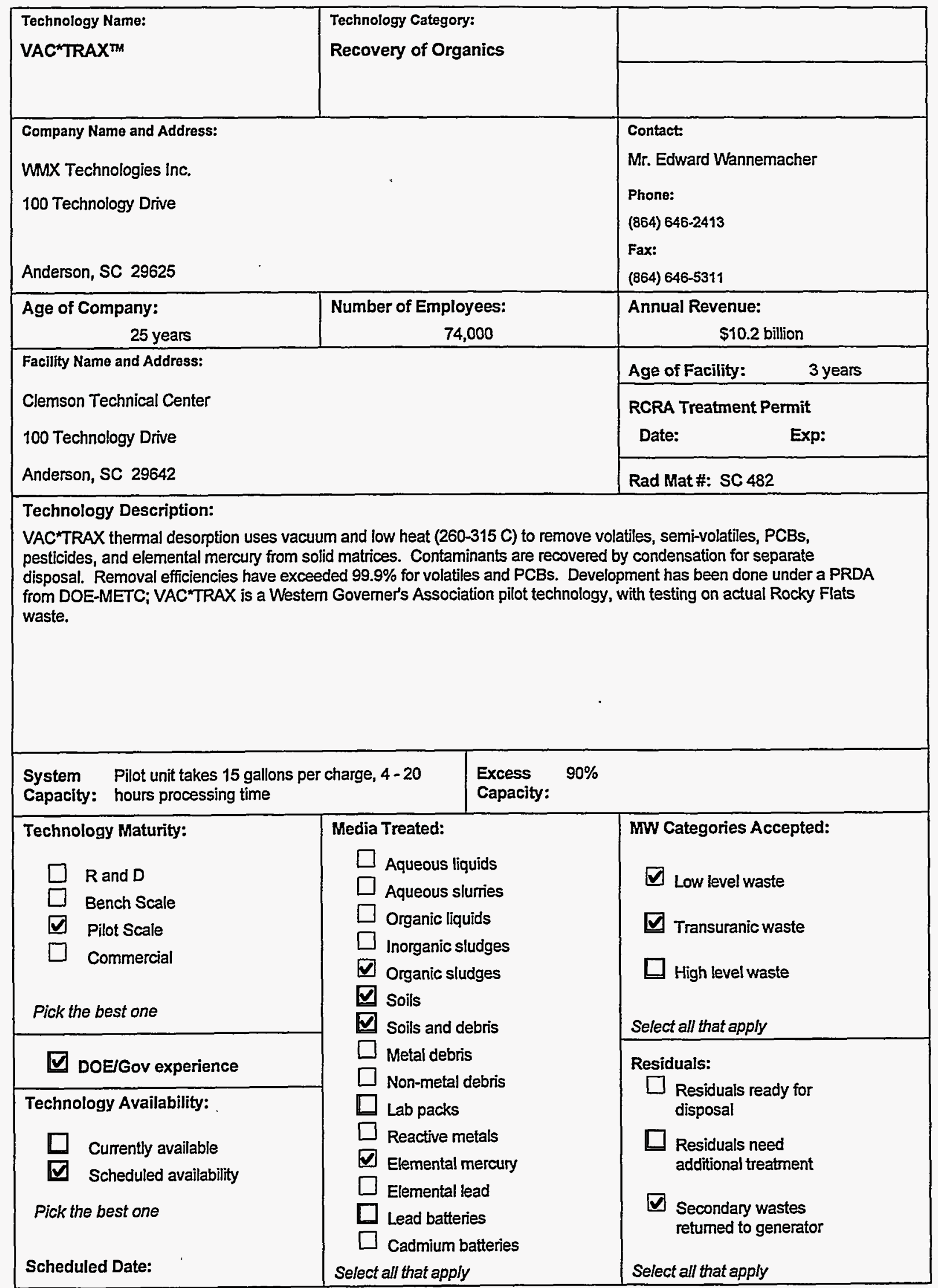

